\title{
Automated data processing and numerical methods for travel-time based hydraulic tomography
}

\author{
Dissertation \\ for the award of the degree \\ "Doctor rerum naturalium" (Dr.rer.nat.) \\ of the Georg-August-Universität Göttingen
}

within the doctoral program Geoscience

of the Georg-August University School of Science (GAUSS)

submitted by

Pengxiang Qiu

from Zhejiang, China

Göttingen, 2020 


\section{Thesis Committee}

Prof. Dr. Thomas Ptak-Fix

Department of Applied Geology, Georg-August-Universität Göttingen

Prof. Dr. Rui Hu

School of Earth Science and Engineering, Hohai University

\section{Members of the Examination Board}

Reviewer: Prof. Dr. Thomas Ptak-Fix

Department of Applied Geology, Georg-August-Universität Göttingen

Second reviewer: Prof. Dr. Rui Hu

School of Earth Science and Engineering, Hohai University

\section{Further members of the Examination Board}

Prof. Dr. Martin Sauter

Department of Applied Geology, Georg-August-Universität Göttingen

Dr. Jannes Kordilla

Department of Applied Geology, Georg-August-Universität Göttingen

Prof. Dr. Thomas Graf

Institute of Fluid Mechanics, Leibniz Universität Hannover

Prof. Dr. Hans Ruppert

Department of Sedimentology and Environmental Geology, Georg-August-Universität Göttingen

Dr. Linwei $\mathrm{Hu}$

Department of Geoscience, Christian-Albrechts-Universität zu Kiel

Date of the oral examination: $15^{\text {th }}$ July 2020 


\section{Affidavit}

I hereby declare that the presented dissertation entitled "Automated data processing and numerical methods for travel-time based hydraulic tomography" has been written independently and with no other sources and aids than quoted.

\section{Pengxiang Qiu}

Göttingen, April 4th, 2020 



\begin{abstract}
Travel time based hydraulic tomography is a useful and promising technique for reconstructing the spatial distribution of aquifer hydraulic properties (e.g., hydraulic diffusivity). Simultaneous Iterative Reconstruction Technique algorithm (SIRT) plays the key role in travel time related inversions. Due to the drawbacks of SIRT implementation in practice, this work proposed a new algorithm, SIRT-Cimmino, which inherits the SIRT structure and embeds Cimmino iteration. Adjustment of incremental correction and introduction of an iteration-dependent relaxation parameter are two major modifications, which enable an appropriate speed of convergence, and the stability of the inversion process. Furthermore, a new result selection rule is employed to determine the optimal iteration step.

SIRT-Cimmino and SIRT are implemented and verified by using three numerical aquifer models with different predefined ("true") diffusivity distributions, where high diffusivity continua are embedded in low diffusivity fields. The first model represents an inclined stratified aquifer, the second model represents a more complex lying Y-shaped aquifer, and the third model is derived from a highly heterogeneous aquifer analogue outcrop. Equivalent porous medium approach is used to simulate pumping tests within the models, and the groundwater drawdown is analyzed to obtain hydraulic travel time. The comparison between the inversion results and the "true" distribution shows that SIRT-Cimmino can reconstruct the continua with better connectivity and more accurate diffusivities than SIRT.

Consequently, travel time based hydraulic tomography is applied at a field test site in Göttingen, Germany. The test site is classified into porous-fractured
\end{abstract}


aquifer by using diagnostic plot. A series of cross-well multi-level pumping tests are performed to derive the drawdown and travel time. Type curve analysis and previous thermal tracer test are used to verify the reconstructions, due to the lack of "true" distribution. The evaluation reveals that the reconstruction obtained by using SIRT-Cimmino has a higher agreement on the structural feature similarity and value accuracy with results from the type curve analysis and thermal tracer test.

In overall, the numerical and field studies prove that (a) the proposed result selection rule can determine the suitable iteration step and (b) the inversion by using SIRT-Cimmino improves the reconstruction quality by showing clear aquifer features and proper diffusivities. 


\section{Acknowledgements}

This dissertation is the work of many people.

First and foremost, I thank my advisor, Prof. Dr.-Ing. Thomas Ptak, who gave a master student with very little hydraulic background a chance to work in his group. He taught me to solve difficult problems and tolerated my mistakes on the field experiment. He taught me not to be satisfied with status quo and always encourages me to write better papers and make better presentations. Now, I am a better writer, presenter, and researcher because of his encouragement.

I am very thankful to my advisor and best friend, Rui, who brought me to this department. As an advisor, he is truly supportive. He always found the time to listen, to discuss, to help and to provide feedback whenever I needed it. He trusted that I can interpret hydraulic problems with my mathematical skill and programming ability. As a friend, we shared the best memory not only on the football pitch but also in the life.

Technical assistance is all-important in the field experiment. For this, I want to greatly acknowledge Steffen Fischer. He has repaired numerous packers and pumps for us in the past three years. With his technical savvy, it was as if there really is no mission impossible.

Field experiment has not been accomplished without excellent collaborators. I am proud to work with Quan, Huichen and Ran. Thanks for the support under any weather conditions! Besides, I will never forget the day when packers fell.

I want to particularly acknowledge my closest collaborator, Quan, with whom I have worked throughout my Ph.D. We designed models, performed field 
experiments, faced difficulties and solved problems together. He is always ready and able to provide efficient help.

To my external research partner, I dedicate my thanks to Linwei Hu from Christian-Albrechts-Universität $\mathrm{zu}$ Kiel. She gave me creative ideas on the development of the methodology.

One of the biggest joys of being in the department was being surrounded by amazing colleagues and officemates. I would like to express my deep gratitude to Xintao, Hongbiao and Yixuan for their helps at any time I need. Special thanks to Reza, Monthon and Viet. Thanks to our different cultural backgrounds, we can talk on various topics and make a lot of happiness. We helped each other and shared enjoyable moments together.

I am fortunate to have you, Reza. Your precise and efficient proof reading has made me fully confident of my manuscript.

I could not have undertaken this journey, far away from home, if not for the unconditional support of my family. They have forgiven my absence from so many family holidays. Throughout my life, their love and support has given me the courage to pursue my interests unapologetically and has made me the person I am today. I hope this dissertation makes them proud!

Finally, nobody has provided more support, encouragement, and love than my wife Betty. Words cannot express how much I thank you!

This work was supported by the CSC scholarship (China Scholarship Council). I also gratefully acknowledge Dr. Iulia Ghergut and Prof. Dr. Thomas Ptak for part of financial support by offering research assistant contract. 


\section{Contents}

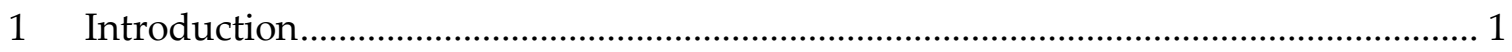

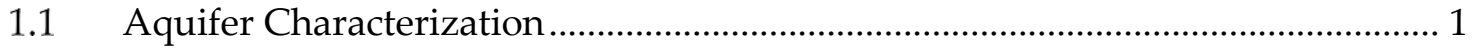

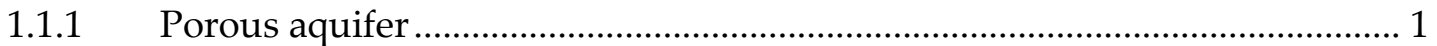

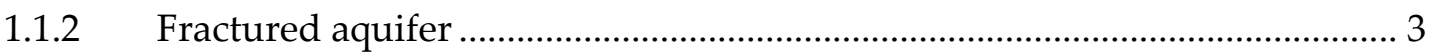

1.1.3 Porous-fractured aquifer …………............................................................... 5

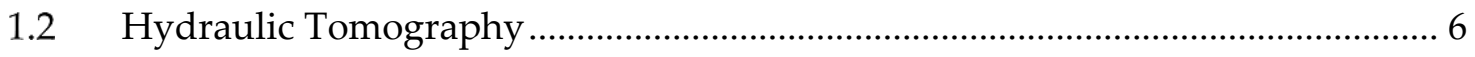

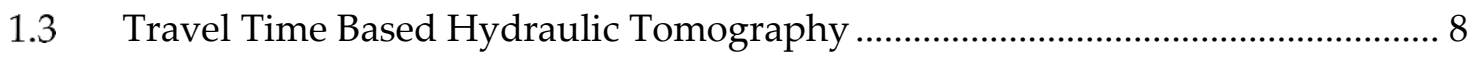

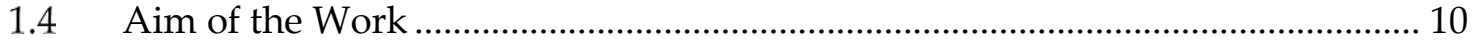

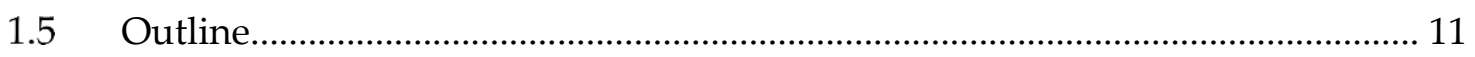

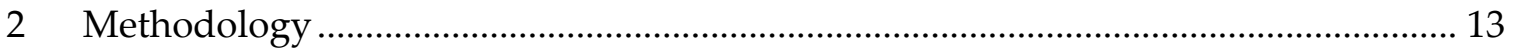

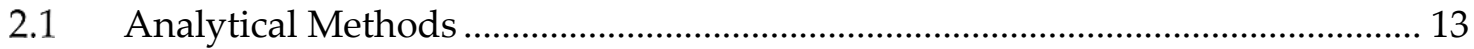

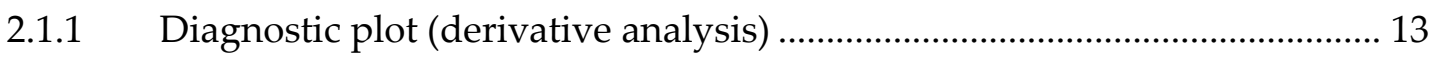

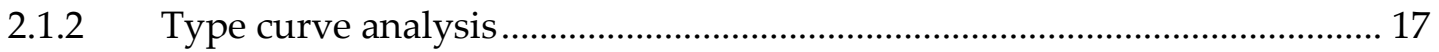

2.2 Travel Time Based Hydraulic Tomography ........................................................ 20

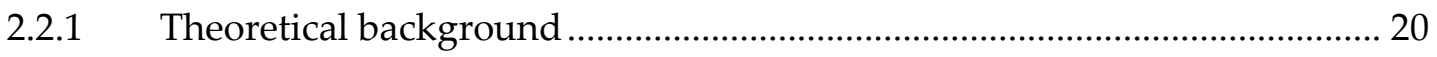

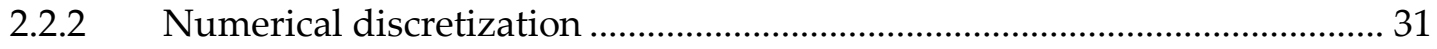

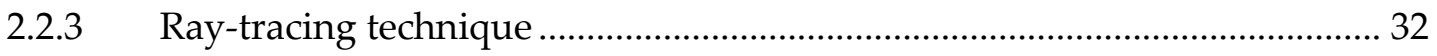

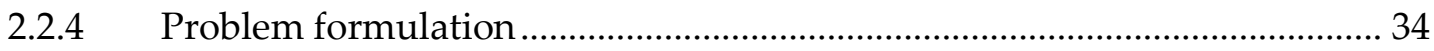

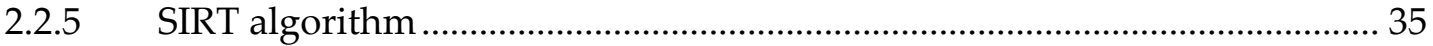

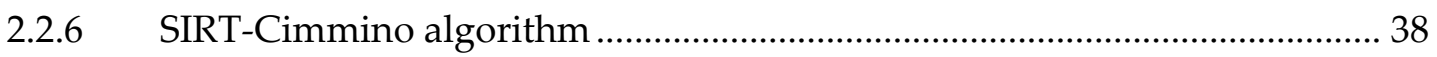

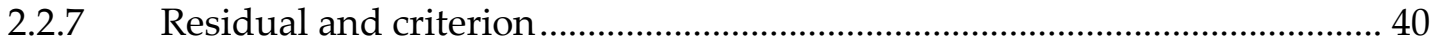

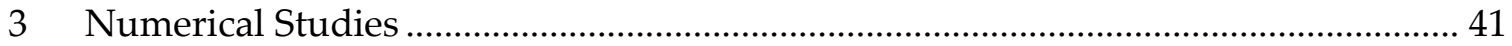

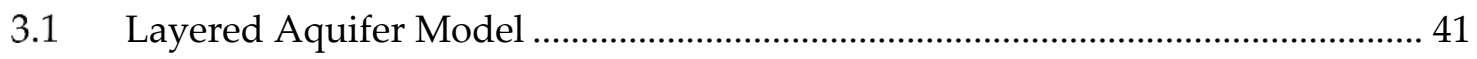

3.1.1 Predefined diffusivity distribution and model setup................................... 41

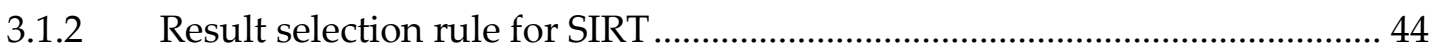

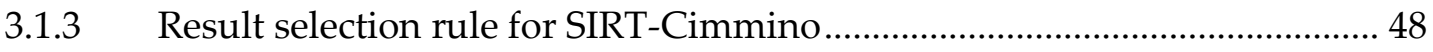

3.1.4 Reconstruction comparison of SIRT and SIRT-Cimmino of the model ...... 49

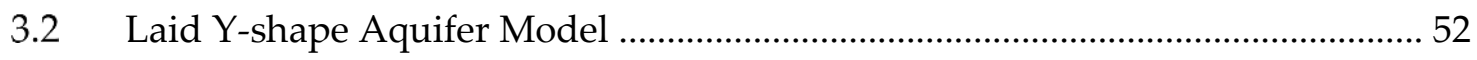

3.2.1 Predefined diffusivity distribution and model setup ................................. 52 
3.2.2 Reconstruction comparison of SIRT and SIRT-Cimmino of the model ...... 53

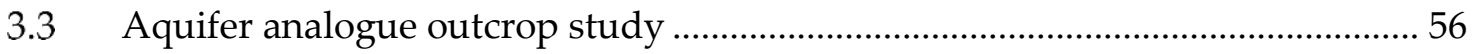

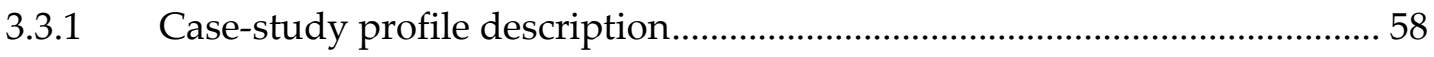

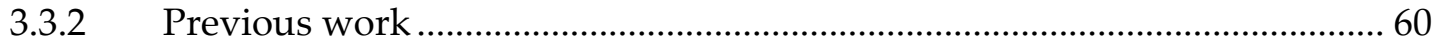

3.3.3 Numerical simulation of short term pumping tests ...................................... 63

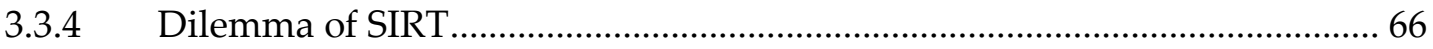

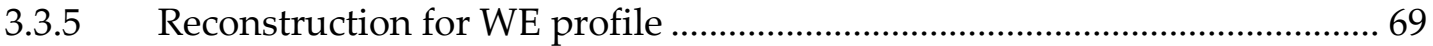

3.3.6 Reconstruction for SN profile ................................................................... 74

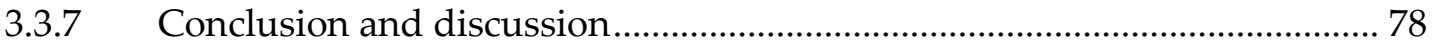

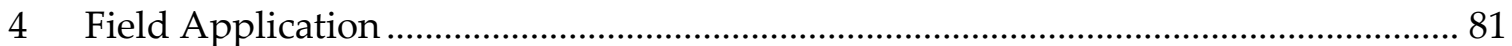

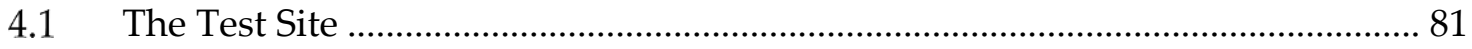

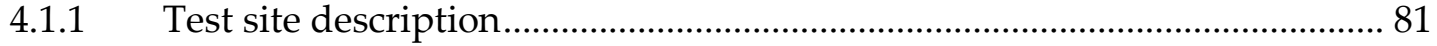

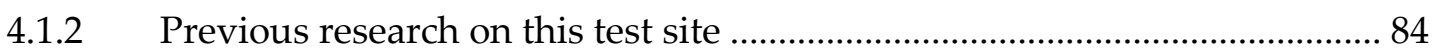

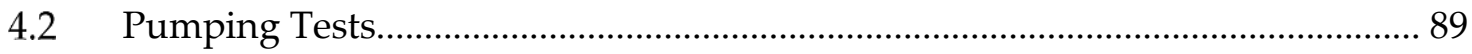

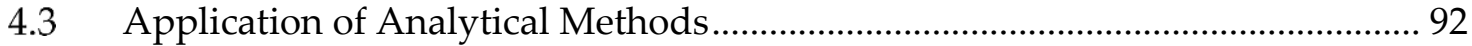

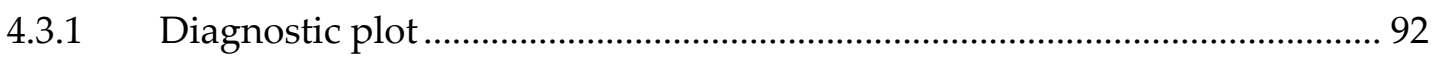

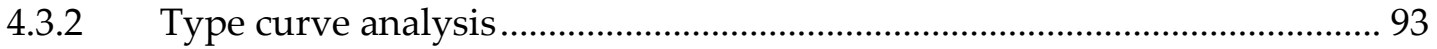

4.4 Application of Travel Time Based Hydraulic Tomography............................... 96

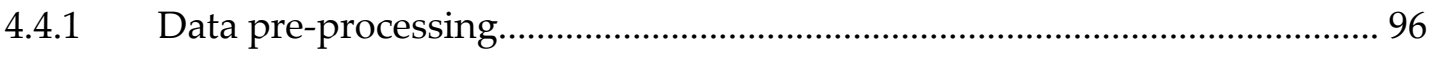

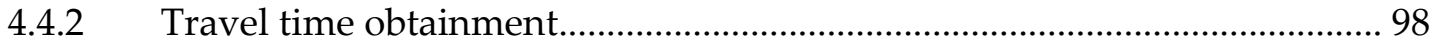

4.4.3 Diffusivity reconstruction by using SIRT algorithm .................................. 99

4.4.4 Diffusivity reconstruction by using SIRT-Cimmino algorithm ................. 101

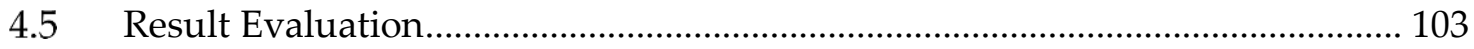

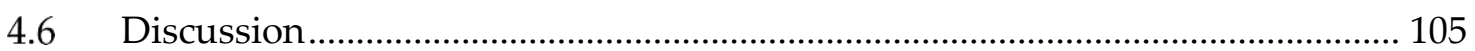

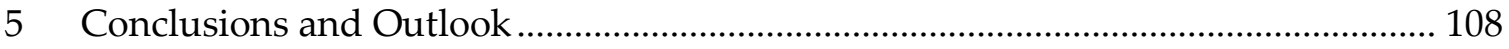

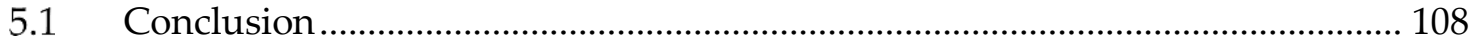

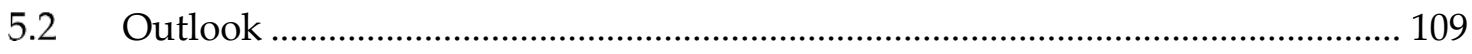

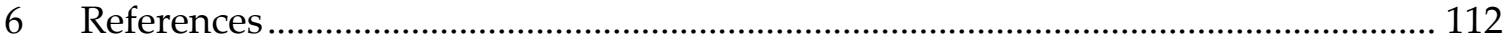

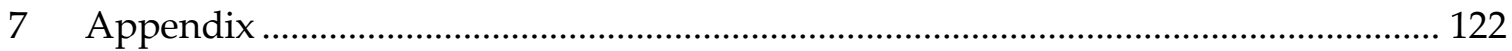

7.1 The transformation of diffusion equation by applying Fourier Transform ..... 122

7.2 Helmholtz equation degrades to the eikonal equation ..................................... 124 


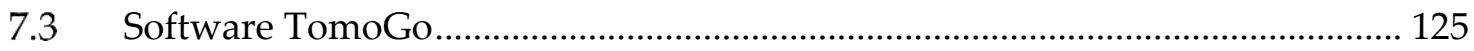

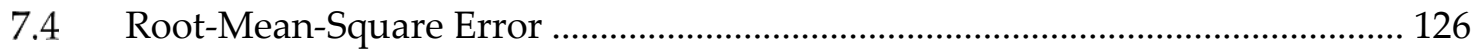

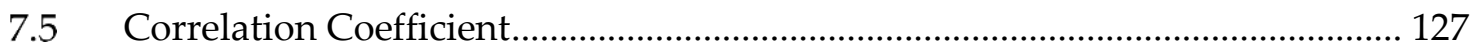

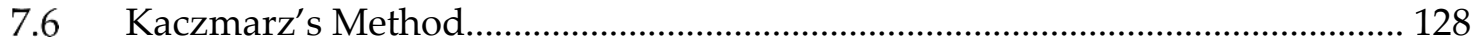

$7.7 \quad$ The travel times for West-East profile .............................................................. 129

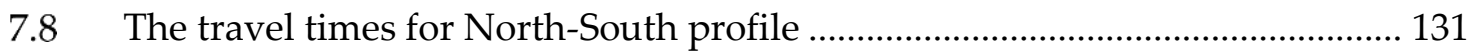





\section{List of Figures}

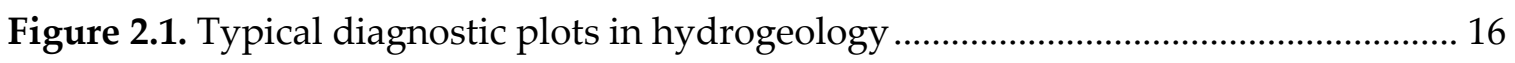

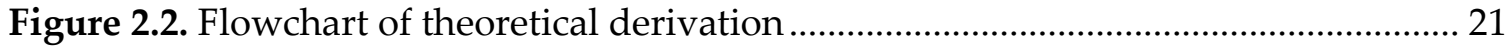

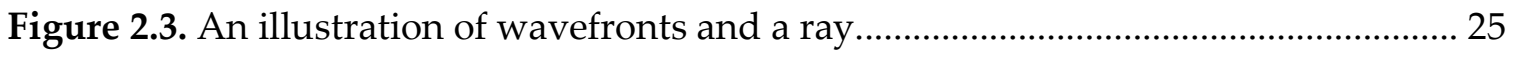

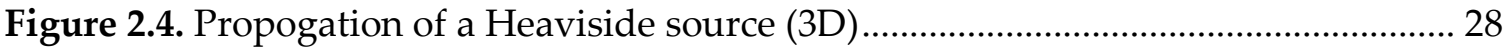

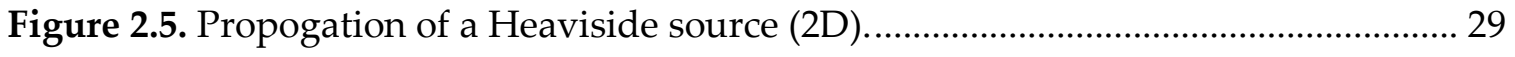

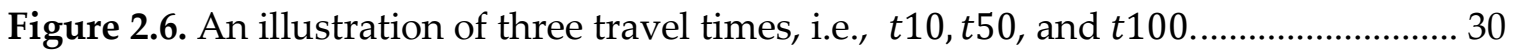

Figure 2.7. Trahectory of a ray travelling through a discrete area .................................... 31

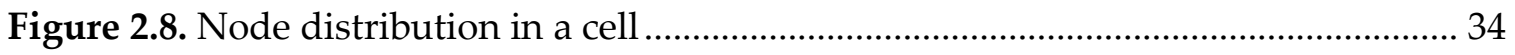

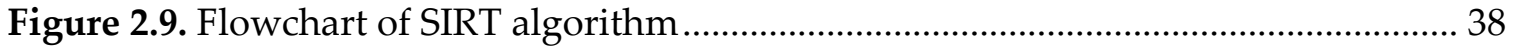

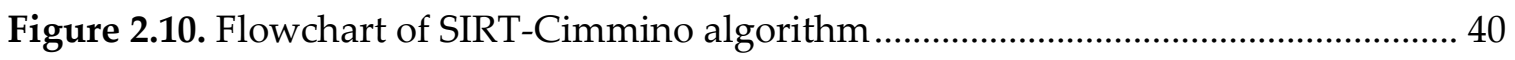

Figure 3.1. Geometry of two 2D axisymmetric models ...................................................... 43

Figure 3.2. The layered model: predefined diffusivity distribution. ................................. 43

Figure 3.3. The layered model: the drawdowns during a simulated pumping test......... 44

Figure 3.4. The layered model: an illustration of travel time computation. ...................... 44

Figure 3.5. The layered model: iteration residuals by using SIRT................................... 45

Figure 3.6. The layered model: SIRT reconstructions at different iteration steps ............. 47

Figure 3.7. The layered model: iteration residuals by using SIRT-Cimmino..................... 49

Figure 3.8. The layered model: predefined diffusivity distribution (for comparison) ..... 50

Figure 3.9. The layered model: result comparison under $8 \times 6$ resolution........................ 50

Figure 3.10. The layered model: result comparison under $8 \times 8$ resolution....................... 50

Figure 3.11. The layered model: result comparison under $12 \times 12$ resolution................... 51

Figure 3.12. The layered model: an illustration of ray trajectories S2R3 and S5R3 ......... 51

Figure 3.13. The laid Y-shape model: predefined diffusivity distribution.......................... 53

Figure 3.14. The laid Y-shape model: predefined diffusivity distribution (for comparison).

Figure 3.15. The laid Y-shape model: result comparison under $8 \times 6$ resolution. ............. 54

Figure 3.16. The laid Y-shape model: result comparison under $8 \times 8$ resolution.............. 55 
Figure 3.17. The laid Y-shape model: result comparison under $12 \times 12$ resolution

Figure 3.18. The laid Y-shape model: an illustration of ray trajectories S1R1, S4R2, S4R8 and S8R8. 56

Figure 3.19. The aquifer analogue outcrop: $3 \mathrm{D}$ distribution of hydraulic diffusivity ...... 57

Figure 3.20. The aquifer analogue outcrop: five-spot well pattern.................................. 59

Figure 3.21. The aquifer analogue outcrop: the diffusivity distribution of WE profile.... 59

Figure 3.22. The aquifer analogue outcrop: the diffusivity distribution of SN profile..... 60

Figure 3.23. The aquifer analogue outcrop: the inversion results in $\mathrm{Hu}$ et al. (2011)...... 62

Figure 3.24. The aquifer analogue outcrop: the notation of screen sections 64

Figure 3.25. The aquifer analogue outcrop: the well arrangement and model overview.65

Figure 3.26. WE profile: an illustration of travel time computation. 65

Figure 3.27. WE profile: iteration residuals by using SIRT. 67

Figure 3.28. WE profile: RMSE of the inversion results by using SIRT. 67

Figure 3.29. WE profile: SIRT reconstructions at different iteration steps 69

Figure 3.30. WE profile: the comparison of inversion results by using SIRT-Cimmino and SIRT . 70

Figure 3.31. WE profile: SIRT-Cimmino reconstructions under high resolutions. ........... 73

Figure 3.32. WE profile: an illustration of ray trajectories from W5 to E4 - E12 ….......... 74

Figure 3.33. SN profile: the comparison of inversion results by using SIRT-Cimmino and SIRT. 75

Figure 3.34. SN profile: SIRT-Cimmino reconstructions under high resolutions............. 77

Figure 3.35. SN profile: an illustration of ray trajectories from S12 to N6 - N12 ............. 78

Figure 3.36. WE profile: the RMSE with respect to the number of cells in the inversion. 79 Figure 3.37. SN profile: the RMSE with respect to the number of cells in the inversion . 80

Figure 4.1. The arrangement of the wells and the location of the field test site 81

Figure 4.2. Sketch of the well construction 83

Figure 4.3. The vertical deflection of wells $\mathrm{N}, \mathrm{O}, \mathrm{S}$, and $\mathrm{M}$ 85

Figure 4.4. The result of the previous thermal tracer test. 87

Figure 4.5. Sketch of the cross-well multi-level short term pumping tests 90 
Figure 4.6. Sketch of double packer system in pumping and observation wells............. 90

Figure 4.7. Schematic of pumping test set-up ................................................................... 91

Figure 4.8. The diagnostic plot for the test O4M4 ............................................................ 93

Figure 4.9. Type curve matching for the test O4M4 …...................................................... 94

Figure 4.10. Travel time computation by using the drawdown in the pumping phase of

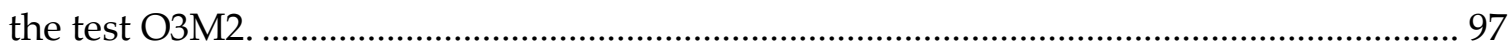

Figure 4.11. Travel time computation by using the drawdown in the recovery phase of the

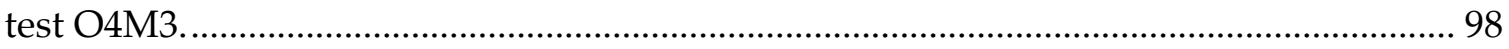

Figure 4.12. The obtained travel times map for the test site............................................. 99

Figure 4.13. Iteration residuals by using SIRT under $9 \times 6$ resolution............................. 100

Figure 4.14. Diffusivity tomograms by using SIRT under $9 \times 6$ resolution at different steps.

Figure 4.15. Iteration residuals by using SIRT-Cimmino under $9 \times 6$ resolution............ 102

Figure 4.16. Field inversion result by using SIRT Cimmino under resolution $9 \times 6 \ldots \ldots .102$

Figure 4.17. Field inversion result by using SIRT Cimmino under resolutions $9 \times 8$ and $9 \times$

9

Figure 4.18. The comparison of the SIRT-Cimmino inversion result under resolution $9 \times 8$ and the previous thermal tracer test result 



\section{List of Tables}

Table 2.1. Analytical models in nonleaky confined aquifers ............................................. 17

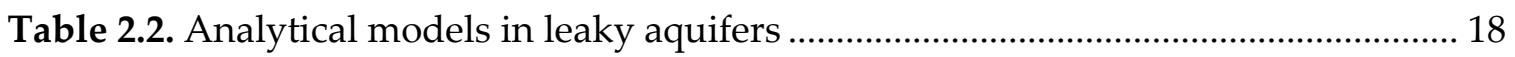

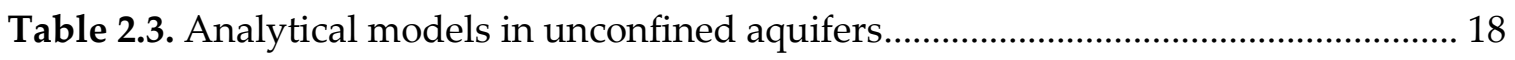

Table 2.4. Analytical models in fractured aquifers ............................................................ 19

Table 3.1. The layered model: optimal iteration step of SIRT under three different

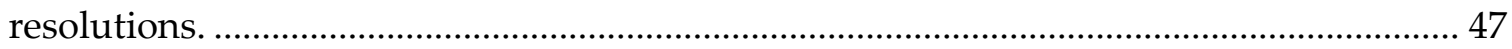

Table 3.2. The layered model: RMSE and correlation coefficient of the results using SIRT-

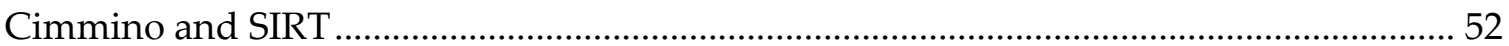

Table 3.3. The laid Y-shape model: optimal iteration step of SIRT under three different resolutions. .53

Table 3.4. The laid Y-shape model: RMSE and correlation coefficient of the results using

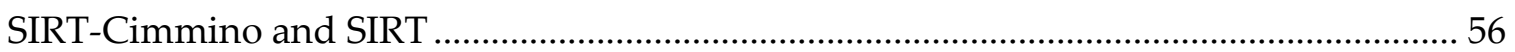

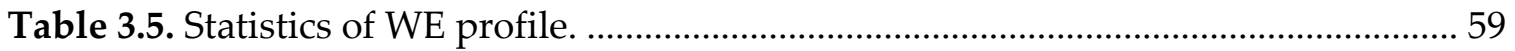

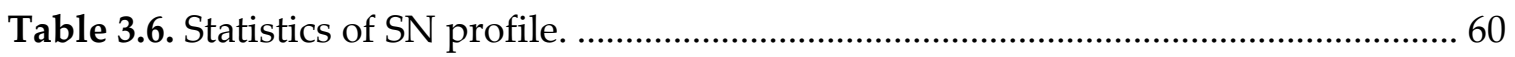

Table 3.7. The comparison of experimental details between $\mathrm{Hu}$ et al. (2011) and this work

Table 3.8. The comparison of average diffusivities of different layers in true distribution of WE profile, the reconstructed distribution by using SIRT (Hu et al. 2011), and the reconstructed distribution by using SIRT-Cimmino.

Table 3.9. The comparison of average diffusivities of different layers in true distribution of SN profile, the reconstructed distribution by using SIRT (Hu et al. 2011), and the reconstructed distribution by using SIRT-Cimmino. 75

Table 4.1. Result of cross-well pumping test at the first screen of five wells. 87

Table 4.2. Result of type curve matching for the cross-well integral pumping test......... 95

Table 4.3. Result of type curve matching for the cross-well multi-level pumping tests.. 95

Table 4.4. Result of type curve matching for the single-well multi-level pumping tests. 96 
Table 4.5. The arithmetic average diffusivities of the results by using SIRT-Cimmino and

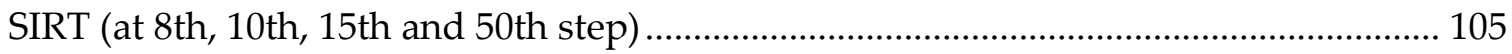

Table 7.1. The travel times of West-East profile .............................................................. 129

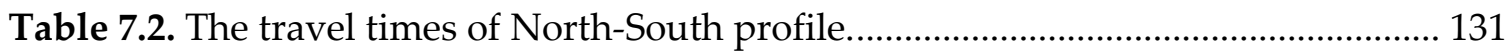




\section{List of Symbols}

\begin{tabular}{|c|c|c|}
\hline [L]: length & {$[\mathrm{M}]$ : mass } & [T]: time \\
\hline$A$ & & matrix related to travel time $(b=A x)$ \\
\hline$A_{0}$ & [L] & $\begin{array}{l}\text { amplitude of the leading term in series expansion used in } \\
\text { asymptotic approach }\end{array}$ \\
\hline$A_{n}$ & [L] & $\begin{array}{l}\text { amplitude of the } n \text {-th term in series expansion used in } \\
\text { asymptotic approach }\end{array}$ \\
\hline$b$ & & vector related to travel time $(b=A x)$ \\
\hline$c_{t}$ & {$\left[\mathrm{~T}^{2} \mathrm{~L} \cdot \mathrm{M}^{-1}\right]$} & total compressibility \\
\hline$D$ & {$\left[\mathrm{~L} \cdot \mathrm{T}^{-2}\right]$} & hydraulic diffusivity \\
\hline$D f$ & {$\left[\mathrm{~L} \cdot \mathrm{T}^{-2}\right]$} & hydraulic diffusivity in fracture \\
\hline$e$ & {$[-]$} & natural logarithm \\
\hline$f$ & & transformation factor \\
\hline$g$ & {$\left[\mathrm{~L} \cdot \mathrm{T}^{-2}\right]$} & gravitational acceleration \\
\hline$i$ & & imaginary unit used in Fourier transform \\
\hline$K$ & {$\left[\mathrm{~L} \cdot \mathrm{T}^{-1}\right]$} & hydraulic conductivity \\
\hline$K f$ & {$\left[\mathrm{~L} \cdot \mathrm{T}^{-1}\right]$} & hydraulic conductivity in fracture \\
\hline$K m$ & {$\left[{\left.\mathrm{~L} \cdot \mathrm{T}^{-1}\right]}^{-1}\right.$} & hydraulic conductivity in matrix \\
\hline$k$ & {$\left[\mathrm{~L}^{2}\right]$} & hydraulic permeability \\
\hline$M$ & & $\begin{array}{l}\text { diagonal matrix used for incremental correction of SIRT- } \\
\text { Cimmino algorithm }\end{array}$ \\
\hline$n$ & {$[-]$} & porosity \\
\hline$N$ & & $\begin{array}{l}\text { diagonal matrix used for incremental correction of SIRT } \\
\text { algorithm }\end{array}$ \\
\hline$P$ & {$\left[\mathrm{M} \cdot \mathrm{L}^{-1} \mathrm{~T}^{-2}\right]$} & pressure \\
\hline$S$ & {$[-]$} & storativity \\
\hline
\end{tabular}




\begin{tabular}{|c|c|c|}
\hline$S_{s}$ & {$\left[\mathrm{~L}^{-1}\right]$} & specific storage \\
\hline$S_{s} f$ & {$\left[\mathrm{~L}^{-1}\right]$} & specific storage in fracture \\
\hline$S_{s} m$ & {$\left[\mathrm{~L}^{-1}\right]$} & specific storage in matrix \\
\hline$S_{y}$ & {$[-]$} & specific yield \\
\hline$s$ & [L] & drawdown (only in Chapter 2.1.1) \\
\hline$s$ & [L] & length of a path in a cell (only in Chapter 2.2.2) \\
\hline$s$ & [L] & path of the propagation of a Dirac signal \\
\hline$T$ & {$\left[\mathrm{~L}^{2} \mathrm{~T}^{-1}\right]$} & hydraulic transmissivity \\
\hline$Q$ & {$\left[\mathrm{~L}^{3} \mathrm{~T}^{-1}\right]$} & pumping rate \\
\hline$t$ & [T] & time \\
\hline$t_{\text {peak }}$ & {$[\mathrm{T}]$} & travel time of the peak of a Dirac signal \\
\hline$w$ & {$\left[\mathrm{~T}^{-1}\right]$} & frequency used in Fourier transform \\
\hline$W$ & & $\begin{array}{l}\text { diagonal matrix used for incremental correction of SIRT } \\
\text { algorithm }\end{array}$ \\
\hline$x$ & & vector related to travel time $(b=A x)$ \\
\hline$x_{1}$ & [L] & coordinate of the source \\
\hline$x_{2}$ & [L] & coordinate of the receiver \\
\hline$\alpha$ & {$\left[\mathrm{T}^{2} \mathrm{~L} \cdot \mathrm{M}^{-1}\right]$} & compressibility of the aquifer skeleton \\
\hline$\beta$ & {$\left[\mathrm{T}^{2} \mathrm{~L} \cdot \mathrm{M}^{-1}\right]$} & compressibility of the groundwater \\
\hline$\lambda$ & {$[-]$} & relaxation parameter \\
\hline$\mu$ & {$\left[\mathrm{M} \cdot \mathrm{L}^{-1} \mathrm{~T}^{-1}\right]$} & viscosity \\
\hline$\rho$ & {$\left[\mathrm{M} \cdot \mathrm{L}^{-3}\right]$} & density of the groundwater \\
\hline$\tau$ & & "eikonal" \\
\hline$\Omega$ & {$\left[\mathrm{L}^{2}\right]$} & 2D domain in numerical discretization \\
\hline$\Omega_{i}$ & {$\left[\mathrm{~L}^{2}\right]$} & $i$-th cell in $\Omega$ \\
\hline
\end{tabular}





\section{Introduction}

Good quality freshwater is a critical issue for most of living organisms. Groundwater, as one of main sources of freshwater, are being either depleted or contaminated due to agricultural, municipal, and industrial activities in many parts of the world. A better management of groundwater resources and reliable predictions of contaminants transport are required to ensure the use of groundwater is sustainable without healthy risk. Numerical modelling has become a widely used tool to solve this problem (Liedl and Ptak 2003). The development of a reliable model needs precise aquifer characterization through various hydraulic investigations.

\subsection{Aquifer Characterization}

Aquifer characterization is broadly defined as processes to evaluate the subsurface structure, hydraulic properties, flow transport, and chemistry in the aquifer (Maliva 2016). The abovementioned features can be used to classify aquifers. In perspective of flow transport, aquifers have three main types: porous, fractured, and porous-fractured aquifers. Moreover, the features are interrelated. For instance, the heterogeneity of hydraulic properties has a great impact on the flow path. This dissertation investigates the spatial distribution of hydraulic diffusivity in the following three aquifer types (porous, fractured, and porousfractured).

\subsubsection{Porous aquifer}

Porous medium is a material containing pores. The material is usually a solid and the pores are filled with liquid or gas. Numerous substances such as soil, cements, ceramics, sand filters, and biological tissues can be considered as porous 
media. This concept is introduced into hydrogeology to describe the flow of groundwater in aquifers. Darcy's law is concluded from the result of experiments on the flow of a fluid through a porous medium. Apart from the composition of a solid and pores, a porous (media) aquifer should have three properties (a) multiphase matter, (b) relatively narrow void space, and (c) interconnected pores (Bear 1972).

For an efficient description of the fluid through porous media, continuum approach is applied to make a passage from the microscopic to the macroscopic level. The approach applies some parameters to enable the interpretation of flow within a porous medium. Porosity $\Phi$ measures the ratio of voids to the total medium volume (Heath 1983). In a porous aquifer, this parameter can be proportional to the hydraulic conductivity $K$, which describes the ability of the aquifer to conduct water through it. Storativity $S$ indicates the ability to release water and is defined as $S=S_{s} \cdot b+S_{y}$, where $S_{s}$ is the specific storage, $b$ is the aquifer thickness, and $S_{y}$ is specific yield. Both $S_{s}$ and $S_{y}$ are related to the porosity (Duffield 2007). Hydraulic diffusivity D, the main parameter in this thesis, is the ratio of $K$ and $S_{s}$ (or the ratio of transmissivity $T$ and $S$ ). In the transient flow equation, $D$ is proportional to the speed of a pressure pulse traveling through the porous aquifer (Shih 2018).

Various hydraulic tests are available to obtain above-mentioned parameters. The most widely used of these tests are slug and pumping tests. In a slug test, water level in a test well is changed suddenly (rise or fall) and the subsequent water level response is measured in the test well and/or surrounding observation wells (Duffield 2007). In a pumping test, the water level is changed by pumping the water out of the test well at a rate. As widely known, slug test only gives information of hydraulic properties in the vicinity of the test well. For a large scale investigation, pumping test is preferred due to the large size of the depression 
cone caused by the pumping process. Slug test does not require additional water injection or extraction, it is therefore widely used in contaminated site surveys (Butler and Zhan 2004, McElwee 2001).

Both tests are interpreted by using an analytical model to match the observed data. Depending on different assumptions, a number of models have been developed in the last eighty years. We take pumping test as an example, Theis (1935) is the first hydrologist to analyze transient flow to a fully penetrating

pumping well in a homogeneous, isotropic and confined aquifer. Cooper and Jacob (1946) used Taylor series to simplify Theis model for greater time values. Hantush (1961a) extended the model in a partially penetrating well. Hantush and Jacob (1955b) started the flow study in leaky aquifers. Due to the rapid development of IT technique, researchers are able to consider more details during the flow movement. The wellbore storage and skin effect was investigated by Agarwal (1970). The delayed yield response in unconfined aquifers was reported by Neuman (1974) and Neuman (1979).

\subsubsection{Fractured aquifer}

A fractured aquifer is interpreted as an aquifer contains sufficient fractures, cracks, joints and faults (Cook 2003). Fractures can be described through their aperture, width, length, orientation, density, surface roughness, etc. Compared with porous media, fractures are locally approximately planar and have wider diameter and larger scale variety.

As fractures play an important role in fluid flow, related research is strongly increased in numerous areas, e.g., petroleum industry (Nelson 2001, Golf-Racht 1982), geothermal reservoir exploration (Bauer et al. 2017, Müller et al. 2010), gas production (Thomas and Bennion 2007, Taherdangkoo et al. 2019, Zhang et al. 
2017), water supply reservoir (Hammond 2017, Carlson 1999), toxic waste disposal (Perkins and Keck 1994, Pusch 1994), high-level nuclear waste repository (Tsang et al. 2015, Braester 1999), mining (Wei et al. 2011, Evans and Pomeroy 1966), geological process (Gudmundsson 2011, Engelder 1987), geotechnical engineering (Vallejo and Liang 1994, Ismail et al. 2011), as well as underground and surface structures (Calayir and Karaton 2005, Saouma et al. 1991).

Characterization of fractured aquifers is one of the main tasks in hydrogeology. Over the last three or four decades, field, laboratory, and modelling studies have been carried out (Bear et al. 1993, NRC 1996, Evans et al. 2001, Faybishenko et al. 2000, Taherdangkoo and Abdideh 2016). Despite of that, a number of problems are raised due to the complexity of physics processes in the fracture system.

Fractured aquifer, as one of main aquifers, is widely distributed and investigated. A number of hydraulic methods (e.g., multi-level pumping test, borehole flowmeter, and tracer approach) can be used to estimate parameters. However, the flow characterization, particularly in well scales (less than 100 meter), is extremely difficult with existing techniques because the hydraulic properties are more related to local heterogeneity (Tsoflias et al. 2001, Clauser 1992). The groundwater flow direction and the preferential flow path can be related more to the orientation of the fractures than to the hydraulic head distributions. In such media, the diffusivity $D$ as the ratio of $K$ and $S_{s}$ becomes a very useful parameter to emphasize the contrast between fractures and matrix, due to the extremely large $K$ and small $S_{s}$ in fractures (Dietrich et al. 2004). 


\subsubsection{Porous-fractured aquifer}

A fractured medium consists two different populations, i.e., fracture zones and matrix blocks. As the filling material (e.g., clay, minerals) in apertures affects the fracture porosity and permeability, Streltsova (1977) classified the fractured medium into (a) purely fractured medium, (b) double porosity medium, and (c) heterogeneous medium. In a purely fractured medium, the porosity and permeability depend on the interconnected fractures as blocks are impervious. In a double porosity medium, both fractures and matrix blocks contribute to groundwater flow, but fractures are the main contributors. In a situation, when fractures are filled with clay or silty material, the fracture permeability is considerably reduced, and such a medium is termed as heterogeneous. The porous-fractured aquifer belongs to the category (b), i.e., double porosity medium. Equivalent porous medium model and double porosity model are two widely used descriptions of the flow in such aquifers.

Equivalent porous medium model is a simple approach in estimating flow and transport, as it does not characterize the fractures. It is demonstrated that flow through fractured medium can be represented by the flow through a porous medium, i.e., by an equivalent continuum model under specific conditions: high fracture density, constant fracture aperture, distributed orientation, and large sample size (Long et al. 1982).

Due to the conflicting conclusion in seepage research under condition of porous media, Barenblatt et al. (1960) first developed double porosity model to quantify flow in a porous-fractured aquifer. Two classes of porosity are described as an equivalent continuum of low-permeability, primary porosity blocks and an equivalent continuum of high-permeability, secondary porosity fractures (Warren and Root 1963). Flow mechanism in double porosity model differs from that in equivalent porous medium. We take pumping test as an example. At early times, 
the removed water is mainly from the fracture because of its high permeability. At intermediate times, the dewatering happens in both fracture and matrix, and there is an exchange process between fracture and matrix (Dietrich et al. 2004). The drawdown remains nearly constant during this period, and this behavior is similar to the delayed yield in the unconfined aquifer (Boulton and Streltsova 1978). At late times, the flow is close to that in a homogeneous porous medium with fracture permeability. Overall, the initial transient stage is the only difference between this model and the ordinary porous medium. Kinds of analytical solutions are derived taking different conditions and assumptions into account. Moench solution addresses the delay effect of fracture skin at the fracture-block interface as a result of mineral deposition and alteration (Moench 1984). Barker solution generalizes the flow dimension to nonintegral values depending on the distribution and connectivity of the conductive fractures (Barker 1988).

The aforementioned analytical solutions are precise mathematical solutions under specific conditions, which can generally not meet in the practice. Furthermore, the analytical solutions provide only the mean of hydraulic parameters and can not give the spatial distribution of hydraulic parameters.

\subsection{Hydraulic Tomography}

Tomography is a technique for imaging sections of objects by using penetrating waves. The variation of wave signals between a transmitter and detector can be analyzed and utilized to reconstruct the distribution of relevant parameters within the investigated object. Since the 1970s, Computed Tomography (CT) has become an important medical tool (Scudder 1978). During a CT experiment, $\mathrm{X}$-rays are absorbed to varying degrees when passing through different human body parts, and the attenuation of the X-ray radiation is measured 
and used to image the scanned body part. Seismic tomography is another application of the tomographic principle. Seismic waves (P-waves, S-waves and surface waves) are generated by earthquake or explosions. They travel through the geological media and are captured in seismic stations. The velocity and the absorption of the waves are strongly dependent on the media properties. Analysis of the travel time and amplitude can be used to infer the subsurface structures (Nolet 2008). Cross-well seismic tomography is an application of seismic tomography for reservoir (e.g., gas or oil) exploration and development by using two drilled wells, where the sources of seismic waves are placed in one well and the receivers are installed in the other well. By using the tomography, the velocity field between the two wells are determined and the reservoir can thus be characterized (Lo and Inderwiesen 1994). A notable difference between CT and seismic tomography is that the $\mathrm{X}$-ray radiation travels through a human body along a straight line, while seismic waves are reflected and refracted in tectonic structures (Tarantola 2005).

Over the past two decades, hydraulic tomography has been developed to determine the spatial distribution of aquifer hydraulic parameters (Hao et al. 2008, Hochstetler et al. 2016, Illman et al. 2008, Illman et al. 2009, Sun et al. 2013, Xiang et al. 2009, Yeh et al. 2014, Zhu and Yeh 2005, Berg and Illman 2011, Berg and Illman 2015, Berg and Illman 2012, Zhao et al. 2015, Cardiff et al. 2013, Cardiff and Barrash 2011, Illman 2014, Illman 2015, Illman et al. 2007, Zha et al. 2018, Zha et al. 2017, Yeh and Liu 2000, Brauchler et al. 2011, Brauchler et al. 2013b, Brauchler et al. 2010, Hu et al. 2015, Hu et al. 2011). The first step of hydraulic tomography is a series of hydraulic tests (e.g., pumping tests and slug tests) conducted in a tomographic configuration. During each test, an interval of the test well is isolated by using some instruments (e.g., a double packer system). Water is allowed to be pumped out or injected into the aquifer only through this interval (e.g., the interval 
between the packers in the double packer system). The hydraulic response (e.g., the groundwater head) are recorded by the pressure transducers, which are placed at different depth of the observation well and isolated by instruments. As the location of the pumping interval varies, a large number of response data can be collected. With appropriate inversion algorithms, the spatial distribution of hydraulic properties can be reconstructed.

Two main geostatistical approaches were frequently used for hydraulic tomography: the Quasi-Linear Geostatistical Approach (QLGA) and the Successive Linear Estimator (SLE). They were developed by Kitanidis (1995) and Yeh et al. (1996), respectively. Both approaches have been evaluated by numerical experiments (Hughson and Yeh 2000, Yeh and Liu 2000, Fienen et al. 2008, Cardiff et al. 2013), laboratory sandboxes (Illman et al. 2007, Liu et al. 2002, Liu et al. 2007, Yeh and Liu 2000, Berg and Illman 2012, Zhao et al. 2015), and used in field tests (Berg and Illman 2011, Berg and Illman 2015, Hochstetler et al. 2016). The results reveal that the approaches are feasible for the characterization of hydraulic parameters $\left(K\right.$ and $\left.S_{s}\right)$. However, the application for 3D inversion face difficulties because of the heavy computational cost caused by the increased number of unknown parameters and higher demands on noise reduction (Zha et al. 2018). Lee and Kitanidis (2014), Zha et al. (2017), and Zha et al. (2018) therefore optimized the parameter representation and the matrix operation to improve the computational efficiency.

\subsection{Travel Time Based Hydraulic Tomography}

Travel Time based Hydraulic Tomography (TTHT) is another approach, which differs from the aforementioned two geostatistical approaches, in that it applies an asymptotic approach to transform the groundwater flow equation into 
the eikonal equation. Ray-tracing techniques can then be utilized to describe the transient pressure propagation and solve the eikonal equation. With this strategy, a line integral is derived, which relates the travel time of the transient pressure signals to a hydraulic diffusivity distribution (Brauchler et al. 2012, Brauchler et al. 2011, Brauchler et al. 2003, Hu 2011, He et al. 2006, Schöniger et al. 2012, Vasco et al. 2000, Hu et al. 2010, Hu et al. 2011). In seismic tomography, the travel time of waves between a source and a receiver have a line integral relationship with the velocity field within the structure. This is similar to $\mathrm{CT}$, where the decimal percent drop in X-ray intensity is linearly related to the attenuation as a line integral (Lo and Inderwiesen 1994). These similarities imply the feasibility of using SIRT and ART algorithms for hydraulic studies (Brauchler et al. 2003, Vasco et al. 2000).

The advantages of TTHT (following the one used for seismic tomography) are rapid data acquisition, boundary independence, high computational efficiency and robustness. Sources can be generated by short-term hydraulic tests (e.g., slug test and short-term pumping test). A steady state is not necessary, and a test can be completed within several minutes. Depends on the field scale, travel times range from seconds to several minutes, thus boundary conditions of the test field can generally not be reached. Huge data sets with thousands of travel times can be handled on a common computer. Following the principles of seismic tomography, a line integral can be derived relating the square root of the pressure response arrival time directly to the square root of the reciprocal of diffusivity. The similarity between the hydraulic line integral and seismic line integral will be exploited by using the same inversion techniques.

Two inversion algorithms are widely used in TTHT: Algebraic Reconstruction Technique (ART) and Simultaneous Iterative Reconstruction Technique (SIRT). ART updates the reconstruction after analysis of a single travel time, while SIRT updates the reconstruction after analysis of the whole set of travel times. 
Compared to ART, SIRT has higher computational stability and is less sensitive to initial values and measurement errors (Gilbert 1972, Gordon et al. 1970, Qiao et al. 2013). Its main disadvantage is higher computational cost. However, considering the rapid development of computational hardware, the relevance of this disadvantage has been greatly reduced. In SIRT, the residual represents the difference between the calculated travel time and the observed travel time. This value reflects the convergence of the algorithm towards a possible solution. Related studies imply that even though the residual may be already convergent, the reconstruction results are highly dependent on the number of iteration steps (NIS), and the applied number of iterations is usually determined (or given) empirically (Hu 2011). To the best of our knowledge, a reliable result selection rule to determine NIS has not yet been developed for TTHT.

\subsection{Aim of the Work}

This thesis focuses on two aspects.

The first focus is the development of the SIRT-Cimmino algorithm coupled with a result selection rule. We have developed this algorithm based on the SIRT algorithm and modified the core iterative part with the idea of Cimmino iteration strategy. The result selection rule aims at solving the NIS problems during the use of SIRT. The algorithm and rule are added as a feature in software TomoGo, which was developed by Qiu et al. (2019). As travel time based hydraulic tomography is advantageous for reconstructing high contrast diffusivity distribution, numerical aquifer models with different high- $D$ features are carried out. The first goal of the numerical studies is the feasibility validation of SIRT-Cimmino in porous aquifers, and the second goal is the comparison of SIRT-Cimmino and SIRT results. 
The second focus is the field application of SIRT-Cimmino. To the best of our knowledge, it would be the first use of travel time based hydraulic tomography in a porous-fractured aquifer. As the true distribution of the field is not available, independent works (i.e., the previous research and our depth-orient hydraulic tests) play a critical role for verification of the inversion result. Apart from that, various experimental uncertainties surrounding the test site can be expected.

\subsection{Outline}

This dissertation is organized as follows.

Chapter 1 introduces the concept of hydraulic tomography as well as its related research methods. As the basis of this thesis, the derivation and utilization of travel time based hydraulic tomography are reviewed.

Chapter 2 gives details of the theoretical background of the travel time based hydraulic tomography, and the discretization of the main equation. The motivation behind the development of SIRT-Cimmino algorithm, and problems often observed in the use of SIRT are described. As the main modifications, the iterative increment and a relaxation parameter are explained. Moreover, a new result selection rule is proposed to play a role as a criterion.

Chapter 3 provides three numerical aquifer models with predefined diffusivity distributions ("truth distributions") to verify the algorithm. The first model represents an inclined stratified aquifer, the second model represents a more complex lying Y-shaped aquifer, and the third model is derived from a highly heterogeneous aquifer analogue outcrop. Based on equivalent porous medium approach, pumping tests are simulated within three models, and the calculated groundwater heads (observations) are analyzed to obtain hydraulic travel times. The SIRT-Cimmino and SIRT algorithms are used to reconstruct the diffusivity distribution. The SIRT tomograms at various steps are compared with 
the true distribution to determine the optimal NIS, while the SIRT-Cimmino uses the result selection rule to determine the optimal NIS. The sensibilities of two algorithms to the NIS are investigated. Distribution similarity and value accuracy are two main measurements to evaluate the inversion results.

Chapter 4 reports the application of SIRT-Cimmino algorithm on a field test site in Göttingen, Germany. The test site and related previous research are first briefly described. For the data achievement from the field, a series of cross-well multi-level pumping tests are carried out. Due to potential high noise during the test, long travel times are computed from the recovery period while small travel times are computed from the pumping period. SIRT-Cimmino and SIRT algorithms are used to reconstruct the diffusivity distribution. To verify the inversion result, we use type curve matching to obtain the diffusivity depth orientated diffusivity variation with appropriate analytical solutions which are suggested by diagnostic plot.

Chapter 5 summarizes the contents of this study and provides outlook for the potential improvement in the future.

Chapters 6 and 7 are reference and appendix, respectively. 


\section{Methodology}

\subsection{Analytical Methods}

\subsubsection{Diagnostic plot (derivative analysis)}

Diagnostic plot (or derivative analysis) is a simultaneous plot of drawdown and the logarithmic derivative of drawdown with respect to time, and is considered a robust tool for evaluating the influence of wellbore storage and skin effect, identifying aquifer type and boundary conditions (Renard et al. 2008). The method was first developed for the petroleum industry (Bourdet et al. 1983, Bourdet et al. 1989, Gringarten et al. 1979, Cinco-Ley and Samaniego-V. 1981) and has been used in the field of hydrogeology for many years (Spane and Wurstner 1993, Beauheim et al. 2004, Sun et al. 2015, Xiao and Xu 2014). The logarithmic derivative is defined as follows

$$
\frac{\partial s}{\partial \ln t}=t \frac{\partial s}{\partial t},
$$

where $s$ is the drawdown in pumping well and $t$ is time. In practice, the derivative using Eq. 2.1 can contain excessive noise. Various numerical differentiation schemes and denoising techniques have been utilized to improve the accuracy of the derivative (Horne 1994, Spane and Wurstner 1993, Duffield 2007).

Compared to the drawdown, the logarithmic derivative is more sensitive to the subsurface properties and it can uncover the subtle variations which are hard to observe in the drawdown. Figure 2.1 illustrates the diagnostic plots of typical aquifer models in log-log graphs.

At the early time of a pumping process, all the pumped water are from the water stored in the well borehole, and the drawdown and the derivative follow the same straight line with a unit slope (Figure 2.1 (b), (c), (i) and (h)). Skin effect is an additional head loss, which occurs during the penetration of groundwater 
into the screen and the gravel pack. For a damaged well (e.g. plugged perforation, mud invasion) or the gravel pack with a lower permeability than the reservoir, the skin effect is positive, and it enhances the hump subsequent to the wellbore storage effect in the derivative curve. For a stimulated well or the gravel pack with a higher permeability than the reservoir, the skin effect is negative, and it weakens the hump. The comparison of the early times of Figure 2.1 (b) and (c) reveals the enhancement of the hump due to the positive skin effect.

The intermediate time of the diagnostic plot is utilized to identify the type of aquifer. We summarize the features of some typical aquifers (confined homogenous aquifer, unconfined aquifer, leaky aquifer, fractured aquifer). The derivative value in a confined homogenous aquifer is a nonzero constant and is shown as a horizontal line in the graph (Figure 2.1 (a)), the mathematical derivation is referred to Renard et al. (2008). The homogeneity in one part of the aquifer causes also horizontal derivative for a period.

The delayed yield in an unconfined aquifer leads to an inflection point (or short interval) in the drawdown curve. This feature is shown as a prominent hole in the derivative (Figure 2.1 (e) and (f)). A similar diagnostic plot can be found in equivalent homogeneous double porosity aquifer, where the hole is caused by the delayed supply from the second component of the aquifer.

Due to the recharge from the low-permeability aquitards, the diagnostic plot of leaky aquifer (Figure 2.1 (d)) is similar to the confined aquifer with a constant head boundary. These two types are difficult to distinguish in the practice, as the difference is that the derivative in a leaky aquifer decreases faster.

The diagnostic plot of fractured aquifers can be very different based on the applied models. Moench (1997) and Barker (1988) developed solutions for double porosity models consisting of low-permeability, primary porosity blocks and high-permeability, secondary porosity fractures (Figure 2.1 (h)). Because of the 
fracture system and the delayed supply from the matrix, a negative slope following the hump and a hole are caused in the derivative. Gringarten and Witherspoon (1972) developed a solution for the model with an infinite conductivity vertical fracture intersecting the pumped well. The drawdown corresponds two times of derivative value in the early time (Figure 2.1 (i)). CincoLey and Samaniego-V. (1981) analyzed flow in a finite conductivity vertical fracture intersecting the pumped well. They demonstrated that the presence of bilinear flow in the fracture system can be identified by a straight line with a slope of 0.25 after the linear flow in the vertical fracture with a slope of 0.5 .

Boundary conditions can be detected and identified by the late time in the diagnostic plot. For instance, the increase of drawdown and derivative indicates a no-flow boundary (Figure 2.1 (b)), while horizontal drawdown and reduction of derivative (to zero) imply a constant head boundary (Figure 2.1 (c)). 
(a)

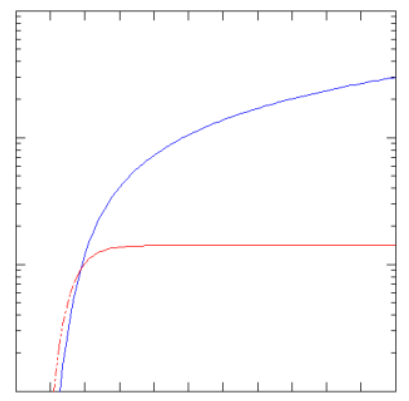

(d)

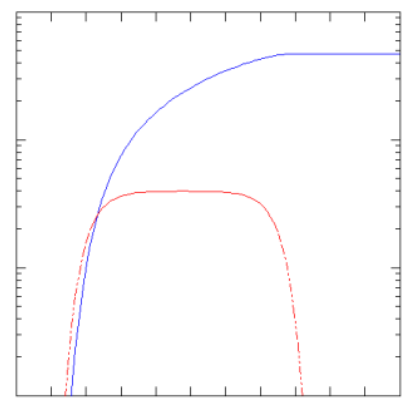

$(\mathrm{g})$

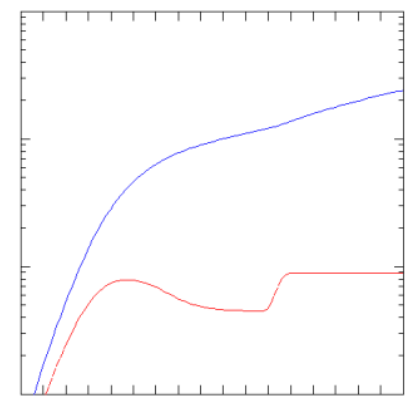

(b)

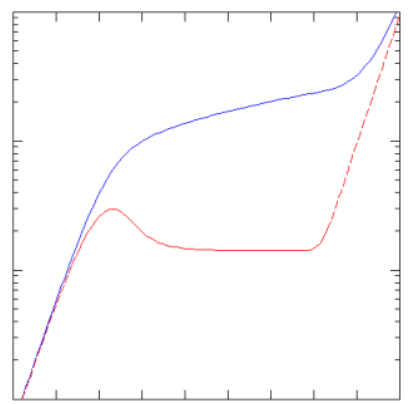

(e)

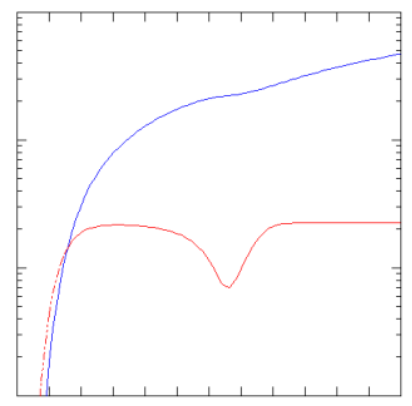

(h)

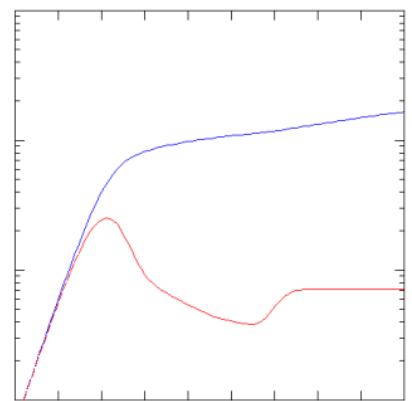

(c)

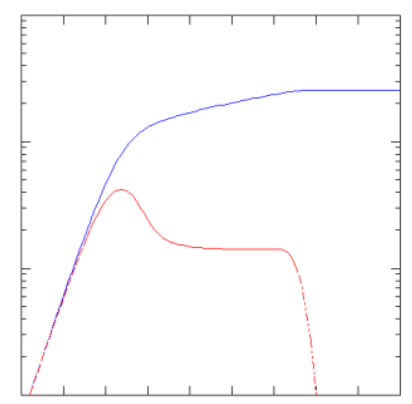

$(\mathbf{f})$

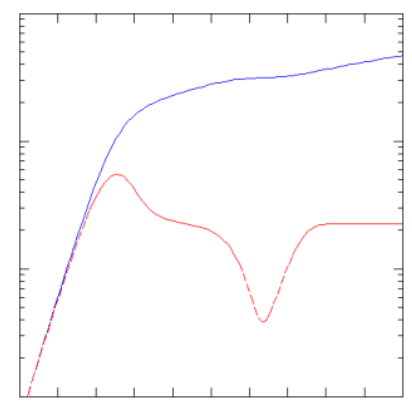

(i)

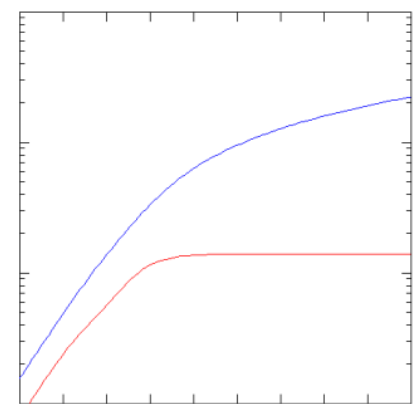

Figure 2.1. Some typical diagnostic plots in hydrogeology by using Aqtesolv. The blue curve is drawdown, the red curve is logarithmic derivative, all curves are illustrated in log-log graphs. (a) Confined aquifer (Theis 1935); (b) confined aquifer with wellbore storage and no-flow boundary (Dougherty and Babu 1984); (c) confined aquifer with wellbore storage, skin effect (positive) and recharge boundary (Dougherty and Babu 1984); (d) leaky aquifer (Hantush and Jacob 1955a); (e) unconfined aquifer (Neuman 1974); (f) unconfined aquifer with wellbore storage (Moench 1997); (g) fractured aquifer (Moench 1997); (h) fractured aquifer with wellbore storage and skin effect (Moench 1997); (i) infinite conductivity vertical fracture aquifer (Gringarten and Witherspoon 1972) 


\subsubsection{Type curve analysis}

A type curve is a visual representation of the analytical response during a test of an interpretation model that characterizes the well and the reservoir. Type curve analysis is aimed to find a type curve that graphically matches the actual response of the well and the reservoir during the test. The parameters, such as permeability, storativity, conductivity and skin factor can be calculated or identified from the "matched" type curve (Gringarten 1987). This method has been used for more than 50 years in the oil industry and for more than 70 years in hydrogeology.

As this thesis focuses on the short-term pumping test, the use of type curve analysis under transient pumping conditions is introduced in this chapter. The first step of the analysis is to find the most appropriate model that represents the flow behavior in the aquifer during the test. The most efficient way to identify the model is use of diagnostic plot, which is explained in detail in the previous subchapter. Since Theis (1935) developed the first rigorous mathematical model, many types of models have been given depending on the condition of the wells and aquifers. We summarize the commonly used models for four most frequently encountered aquifer types, namely, confined (Table 2.1), leaky (Table 2.2), unconfined (Table 2.3) and fractured aquifers (Table 2.4).

Table 2.1. Analytical models in nonleaky confined aquifers ${ }^{1}$

\begin{tabular}{cccc}
\hline & $\begin{array}{c}\text { wellbore } \\
\text { storage }\end{array}$ & skin & Description \\
\hline Theis (1935) & no & no & $\begin{array}{c}\text { It is well known as the first analytical } \\
\text { model. }\end{array}$ \\
$\begin{array}{c}\text { Cooper and Jacob } \\
(1946)^{2}\end{array}$ & no & no & $\begin{array}{c}\text { This model is the late-time } \\
\text { approximation of Theis model. Small } \\
\text { well diameter and large time are } \\
\text { assumed. Simplicity is the main } \\
\text { advantage. }\end{array}$ \\
\hline
\end{tabular}




\begin{tabular}{cccc}
\hline $\begin{array}{c}\text { Papadopulos and } \\
\text { Cooper (1967) }\end{array}$ & yes & no $^{3}$ & $\begin{array}{c}\text { A less restrictive boundary condition is } \\
\text { added at the test well. }\end{array}$ \\
\hline Barker $(1988)^{3}$ & yes & yes & $\begin{array}{c}\text { Flow dimension is introduced for } \\
\text { characterization. }\end{array}$ \\
\hline $\begin{array}{c}\text { Dougherty and } \\
\text { Babu (1984) }\end{array}$ & yes & yes & $\begin{array}{c}\text { The model accounts for (a) Partial } \\
\text { penetration and (b) hydraulic } \\
\text { conductivity anisotropy ratio. }\end{array}$ \\
\hline
\end{tabular}

${ }^{1}$ Estimated parameters are transmissivity and storativity of the aquifer. ${ }^{2}$ This solution is also known as straight-line method. ${ }^{3}$ The skin effect can be integrated into effective radius. ${ }^{4}$ Single porosity is assumed for the model in confined aquifers.

Table 2.2. Analytical models in leaky aquifers ${ }^{1,2}$

\begin{tabular}{|c|c|c|c|}
\hline & $\begin{array}{l}\text { wellbore } \\
\text { storage }\end{array}$ & skin & Description \\
\hline $\begin{array}{l}\text { Hantush and } \\
\text { Jacob }(1955 b)\end{array}$ & no & no & $\begin{array}{c}\text { Aquitards are incompressible (no } \\
\text { storage) }\end{array}$ \\
\hline $\begin{array}{l}\text { Hantush }(1961 b), \\
\text { Hantush }(1961 a)^{2}\end{array}$ & no & no & $\begin{array}{l}\text { (a) Partial penetrating well is accounted, } \\
\text { and (b) aquitards are incompressible (no } \\
\text { storage) }\end{array}$ \\
\hline $\begin{array}{l}\text { Neuman and } \\
\text { Witherspoon } \\
\quad(1969)\end{array}$ & no & no & $\begin{array}{l}\text { The model accounts for (a) Drawdown } \\
\text { in the unpumped aquifer and estimates } \\
\text { (b) uniform storativity and vertical } \\
\text { conductivity in aquitards. }\end{array}$ \\
\hline Moench 1985 & yes & yes & $\begin{array}{l}\text { Storativities and vertical conductivities } \\
\text { in two aquitards can be individually } \\
\text { estimated. }\end{array}$ \\
\hline \multicolumn{4}{|c|}{$\begin{array}{l}{ }^{1} \text { Basic estimated parameters: transmissivity and storativity of the aquifer; vertical hydraulic } \\
\text { conductivity of aquitards. }{ }^{2} \text { Assuming zero conductivity in aquitards, Hantush model can be } \\
\text { used for confined aquifers with partial penetrating well. }\end{array}$} \\
\hline \multicolumn{4}{|c|}{ Table 2.3. Analytical models in unconfined aquifers ${ }^{1,2}$} \\
\hline & $\begin{array}{l}\text { wellbore } \\
\text { storage }\end{array}$ & skin & features \\
\hline Neuman (1974) & no & no & Partial penetration is accounted. \\
\hline Moench (1997) & yes & yes & Partial penetration is accounted. \\
\hline $\begin{array}{l}\text { Tartakovsky and } \\
\text { Neuman (2007) }\end{array}$ & no & no & $\begin{array}{l}\text { The model accounts for (a) partial } \\
\text { penetration and (b) unsaturated flow } \\
\text { above the water table. }\end{array}$ \\
\hline
\end{tabular}


${ }^{1}$ Basic estimated parameters: transmissivity, elastic storage coefficient, specific yield and vertical hydraulic conductivity. ${ }^{2}$ Theis and Jacob-Cooper models can be used in unconfined aquifers by a replacement $\mathrm{s}^{\prime}=\mathrm{s}-\mathrm{s} 2 / \mathrm{b}$, where $\mathrm{s}^{\prime}$ is corrected drawdown, $\mathrm{s}$ is the observed drawdown, and $\mathrm{b}$ is the saturated thickness (Kruseman and Ridder 1994).

Table 2.4. Analytical models in fractured aquifers

\begin{tabular}{|c|c|c|c|}
\hline & $\begin{array}{l}\text { wellbore } \\
\text { storage }\end{array}$ & skin & features \\
\hline Barker (1988) & yes & yes & $\begin{array}{l}\text { This solution uses double porosity } \\
\text { model and flow dimension. }\end{array}$ \\
\hline Moench (1988) & yes & yes & $\begin{array}{l}\text { Double porosity model is assumed with } \\
\text { slab or spherical matrix blocks and } \\
\text { fracture skin. Partial penetration is } \\
\text { accounted. }\end{array}$ \\
\hline $\begin{array}{l}\text { Gringarten and } \\
\text { Witherspoon } \\
\text { (1972) }\end{array}$ & no & no & $\begin{array}{l}\text { The test well is intersected with a single } \\
\text { vertical plane fracture. }\end{array}$ \\
\hline $\begin{array}{l}\text { Gringarten and } \\
\text { Ramey (1974) }\end{array}$ & no & no & $\begin{array}{l}\text { The test well is intersected with a single } \\
\text { horizontal plane fracture. }\end{array}$ \\
\hline
\end{tabular}

Three advantages of type curve analysis are summarized.

- Due to the simplicity, various number of software and programs are available to implement this method. The high development of IT technique significantly reduces the time cost on the curve fitting process.

- All data in the test (pumping and recovery periods) are used. The influence of random errors, mainly caused by electronic noise in the circuit of electrical devices during the test, can be limited.

- The reliability of the estimated parameters is evaluated by goodness of fit between the analytical curve and observation drawdowns. This evaluation can be carried out visually.

Analytical models are found on the basis of very restrictive assumptions, which lead to following disadvantages. 
- Due to the assumption of homogeneity and isotropy, the estimated parameters represent the whole aquifer. The heterogenous and anisotropic information can not be obtained.

- It is assumed that the water is released instantaneously from the storage when the hydraulic head declines. This assumption causes the drawdown deviation in the beginning phase.

- Darcy's law is used to describe the flow mechanics in models, where aquifers are approached through equivalent porous media. Darcy's law is only valid for laminar flow, but turbulence can not be ignored in certain materials, e.g., very coarse-grained sediment. Hence more head loss is caused and contributes to the deviation.

- In practice, the aquifer can not be clearly classified through the use of diagnostic plot. More than one model can be used to interpret the investigated aquifer. This non-uniqueness leads to a parameter misestimation.

To overcome the drawbacks, we suggest combining the type curve analysis with some other geological surveys, for example, core sampling-based geological, mineralogical and structure geological research work, as well as geophysical measurements.

\subsection{Travel Time Based Hydraulic Tomography}

\subsubsection{Theoretical background}

We derive the following line integral in a constant rate cross-well pumping test

$$
\sqrt{t_{\text {peak }}\left(x_{2}\right)}=\frac{1}{\sqrt{c}} \int_{x_{1}}^{x_{2}} \frac{d s}{\sqrt{D(s)}}
$$


where $x_{1}$ is the pumping point, $x_{2}$ is the observation point, $c$ is a dimensional dependent parameter, $D$ is the hydraulic diffusivity, and $t_{\text {peak }}$ is the time when the slope of the drawdown reaches its maximum value (at observation point $x_{2}$ ). Figure 2.2 shows the theoretical derivation briefly.

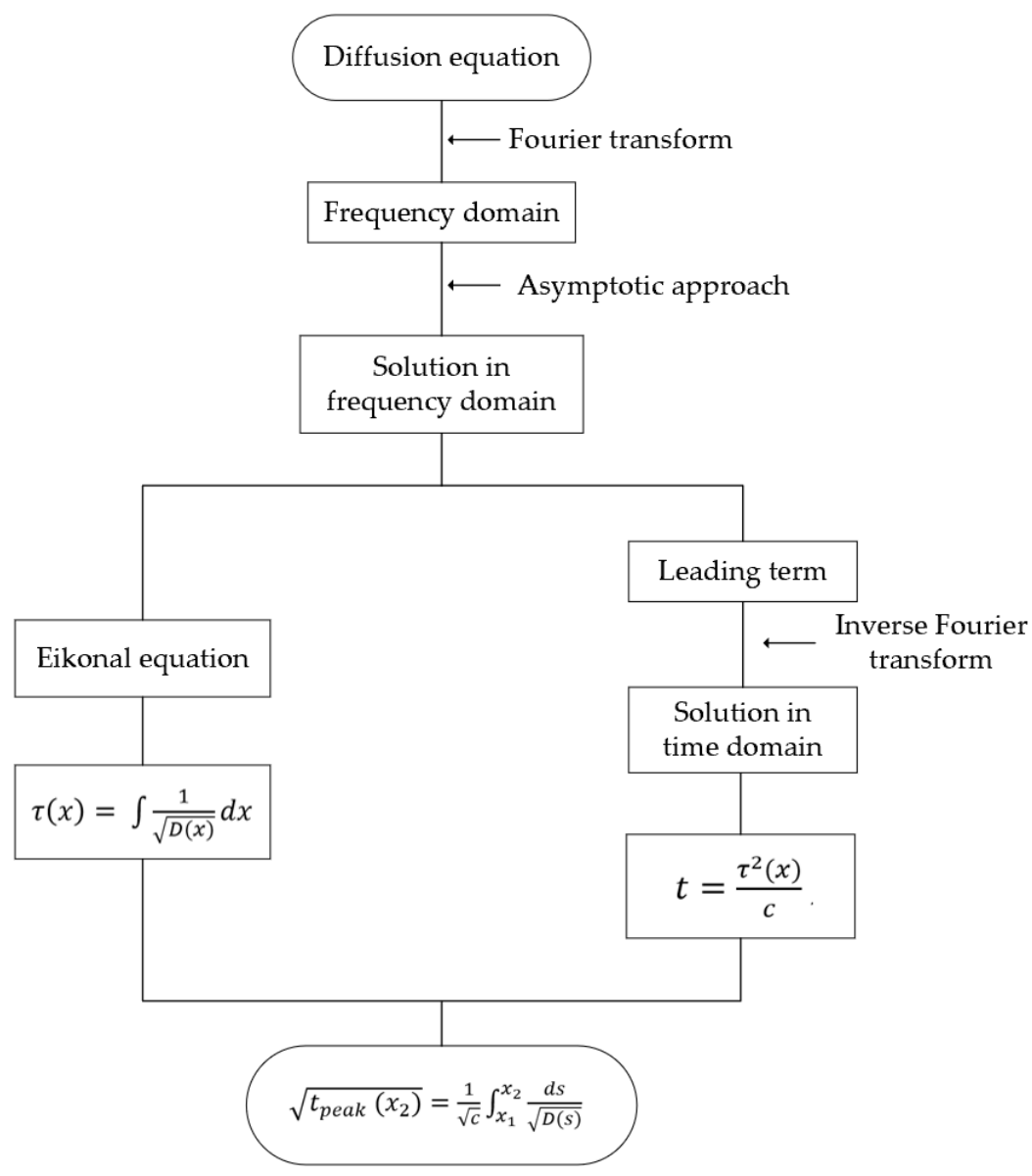

Figure 2.2. Flowchart of theoretical derivation.

We start from the diffusion equation for transient pressure response in a heterogeneous, fully saturated porous medium. It is given with boundary conditions (Streltsova 1988),

$$
\begin{gathered}
n(x) \mu c_{t} \frac{\partial P(x, t)}{\partial t}-\nabla \cdot(k(x) \nabla P(x, t))=0, \\
P( \pm \infty, t)=0,
\end{gathered}
$$


where $P(x, t)$ is the pressure, $n(x)$ is the porosity, $k(x)$ is the permeability, $\mu$ is the viscosity, and $c_{t}$ is the total compressibility. For convenience, $P, n$, and $k$ denote $P(x, t), n(x)$ and $k(x)$, respectively.

We recall some definitions from hydrogeology. The hydraulic conductivity $K$ is a measure of the capacity of an aquifer to transmit water and is determined from Darcy's law. It is related to the hydraulic permeability $k$ through

$$
K=k \frac{\rho g}{\mu} .
$$

In a confined aquifer, specific storage is a characterization of the capacity of an aquifer to release groundwater, and is defined as

$$
S_{s}=\rho g(\alpha+n \beta) \text {, }
$$

where $\rho$ is the density of groundwater, $g$ is the acceleration of gravity, $\alpha$ is the compressibility of the aquifer skeleton, $n$ is the porosity, and $\beta$ is the compressibility of groundwater (Duffield 2007). Hydraulic diffusivity $D$ is defined as the ratio of conductivity and specific storage

$$
D=\frac{K}{S_{S}} .
$$

Note that both $K$ and $D$ are spatial variables and denote $K(x)$ and $D(x)$, respectively.

In a confined and heterogeneous aquifer, the skeleton is assumed to be incompressible ( $\alpha=0$ in Eq. 2.6). The diffusion equation Eq. 2.3 can be written simply in terms of conductivity and diffusivity by using the definitions in Eq. 2.5, Eq. 2.6 and Eq. 2.7

$$
\triangle P+\frac{\nabla k}{k} \cdot \nabla P-\frac{1}{D} \frac{\partial P}{\partial t}=0 .
$$

Due to the complex of solving partial differential equations in the time domain, we transform the equation into the frequency domain by first using the Fourier 
transform, then using an asymptotic series to approach the solution (in the frequency domain), and lastly transforming the solution back to the time domain by using the inverse Fourier transform. The detailed derivation of the transformation into frequency domain using the Fourier transform is available in Appendix 7.1, and the equation in the frequency domain is derived

$$
\triangle \widehat{P}+\frac{\nabla k}{k} \cdot \nabla \hat{P}-\frac{1}{D} i \omega \hat{P}=0,
$$

where $\hat{P}$ notates the Fourier transform of $P$.

An asymptotic approach is proposed to solve Eq. 2.9 by Fatemi et al. (1995). They assume the solution can be approached in the form of an asymptotic series

$$
\hat{P} \approx e^{-\sqrt{-i \omega} \tau(x)} \sum_{j=0}^{\infty} \frac{A_{j}(x)}{(\sqrt{-i \omega})^{j}} .
$$

Virieux et al. (1994) explained the expansion differs from the conventional asymptotic expansion, as it uses $\sqrt{-i \omega}$ instead of $\omega$ in order to reduce the solution in a homogeneous medium. This asymptotic expression is not unique. We can use other expressions (Dingle 1973), for example,

$$
\hat{P} \approx \omega^{-\varepsilon} e^{i \omega \tau(x)} \sum_{j=0}^{\infty} \frac{A_{j}(x)}{\omega^{j}},
$$

where the constant $\varepsilon$ is chosen to make the leading-order equation nontrivial (Chapman et al. 1999).

The idea behind the asymptotic approach is the WKB theory, which is a method for approximating the differential equation whose highest derivative is multiplied by a small parameter. The strategy of WKB is to substitute a series expansion into the original equation (the right side of the equation is always zero), compare the behavior of the terms, as the small parameter approaches to zero, then obtain the dominant balance which usually is related to the leading term in the series expression (Dingle 1973). In our case, let all terms in Eq. 2.9 be divided by 
$\omega$, so that the coefficient of $\triangle \hat{P}$ becomes $\omega^{-1}$ which can be considered the small parameter, since $\omega^{-1} \rightarrow 0$ when $\omega \rightarrow \infty$. We then apply the WKB theory and obtain Eq. 2.10. The solution is called a high frequency solution since the solution is obtained when $\omega \rightarrow \infty$. Interested readers are referred to Dingle (1973) and Olver (1997).

We substitute Eq. (2.1.9) into Eq. (2.1.8) to obtain an expression with an infinite number of terms, each containing $\sqrt{-i \omega}$ to some order. Vasco et al. (2000) considered the sets of terms for any given order and studied the terms of $(\sqrt{-i \omega})^{2}$, obtaining the following equations for $\tau(x)$

$$
\begin{gathered}
\nabla \tau(x) \cdot \nabla \tau(x)-\frac{1}{D(x)}=0, \\
\nabla \tau(x)=\frac{1}{\sqrt{D(x)}}, \\
\tau(x)=\int \frac{1}{\sqrt{D(x)}} d x,
\end{gathered}
$$

where the $\tau(x)$ is the so-called "eikonal" and Eq. 2.12 is known as the eikonal equation.

In order to understand the physical meaning of the eikonal equation, we introduce two concepts: wavefront and ray. A wavefront is the set of all points where a wave has the same phase at a given instant of time. A ray meanwhile is a geometrical vector that is perpendicular to the wavefront. In other words, a ray path shows the propagation of a wavefront (Figure 2.3). 


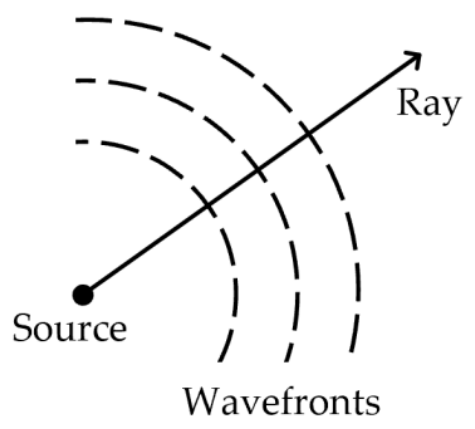

Figure 2.3. An illustration of wavefronts and a ray.

In physics, the Helmholtz equation describes mechanical waves, for example, light (Born and Wolf 1999), sound (Bruneau 2006) and seismic waves (Lo and Inderwiesen 1994). As Appendix 7.2 shows, the high frequency Helmholtz equation degrades to the eikonal equation, therefore the solution of the eikonal equation describes the propagation of a high frequency wave. Eq. 2.14 represents a ray passing through a point $x$ in a medium at time $\tau(x)$ with velocity $\sqrt{D(x)}$ (Zhao 2004).

The relationship between the eikonal and the hydraulic parameter diffusivity is established in Eq. 2.14, we will now build the link between the eikonal and transient pressure. We transform the leading term

$$
\widehat{P}=e^{-\sqrt{-i \omega} \tau(x)} A_{0}(x)
$$

directly back to the time domain by using the inverse Fourier transform. A detailed derivation of this process is described by Vasco et al. (2000). For an impulse source, the corresponding solution in the two-dimensional case is (Kulkarni et al. 2001)

$$
P=A_{0}(x) \frac{\tau(x)}{2 \sqrt{\pi} t} e^{-\frac{\tau^{2}(x)}{4 t}}
$$

and in the three-dimensional case is

$$
P=A_{0}(x) \frac{\tau(x)}{2 \sqrt{\pi t^{3}}} e^{-\frac{\tau^{2}(x)}{4 t}} .
$$


We consider the maximized pressure response at a fixed position $x$, which indicates the derivative of $P$ with respect to time $t$ equals 0 :

$$
\frac{\partial P(x, t)}{\partial t}=0 \rightarrow t_{\max }=\frac{\tau^{2}(x)}{c},
$$

where $c$ is a dimension dependent coefficient. For the two-dimensional case $c$ is 4 and for the three-dimensional case $c$ is 6 (Kulkarni et al. 2001, Vasco et al. 2000). The eikonal is now linked to "time", which can be determined by the pressure curve. The theoretical work of domain transformation may appear complicated to a non-mathematician, Kulkarni et al. (2001) presented the same result based on the well-known Theis drawdown equation, which is much more understandable to the majority of readers. Even so, the derivation with asymptotic approach offers more physical significance.

If we assume maximized pressure responses are observed at position $x_{1}$ and time $t_{1}$, and also at $x_{2}$ and time $t_{2}$, the relationship between $t$ and $D$ can be derived by combining Eq. (2.1.13) and Eq. (2.1.16),

$$
\sqrt{t_{2}-t_{1}}=\frac{1}{\sqrt{c}} \int_{x_{1}}^{x_{2}} \frac{1}{\sqrt{D(x)}} d x .
$$

Note that this line integral is only related to diffusivity $D(x)$. The derived response is caused by an impulse source that can be described as a delta function. In hydraulic tests, pneumatic slug tests cause a pressure drop in a very short time and therefore can be considered an impulse source. Assuming that a cross-well slug test takes place at time zero and position $x_{1}$, and the drawdown peak (maximized pressure) is at time $t_{\text {peak }}$ and observation position $x_{2}$, then the Eq. 2.19 is written as

$$
\sqrt{t_{\text {peak }}}=\frac{1}{\sqrt{c}} \int_{x_{1}}^{x_{2}} \frac{1}{\sqrt{D(x)}} d x .
$$


Due to the scale limitation of slug test, we are more interested in pumping test. The constant rate pumping test commences at time zero and continues at a constant rate. This process behaves as a step function, which is categorized as a Heaviside source. To investigate the pressure response to a Heaviside source, we use the fact that the derivative of a step function is a delta function, and obtain the pressure response in the two-dimensional case

$$
\frac{\partial P}{\partial t}=A_{0}(x) \frac{\tau(x)}{2 \sqrt{\pi} t} e^{-\frac{\tau^{2}(x)}{4 t}},
$$

and in the three-dimensional case

$$
\frac{\partial P}{\partial t}=A_{0}(x) \frac{\tau(x)}{2 \sqrt{\pi t^{3}}} e^{-\frac{\tau^{2}(x)}{4 t}} .
$$

A detailed derivation is covered by Vasco et al. (2000). It is interesting that Eq. 2.21 and Eq. 2.22 are of the same form as Eq. 2.16 and Eq. 2.17, respectively. The difference is on the left side, where the pressure response from an impulse source corresponds to the slope of the pressure response from a Heaviside source. For convenience, we use "slope" to denote the first derivative of pressure with respect to time. We calculate the second derivative of pressure with respect to time to obtain the time when the slope is maximized (i.e., $\frac{\partial P}{\partial t}$ is maximized),

$$
\frac{\partial^{2} P(x, t)}{\partial t^{2}}=0 \rightarrow t=\frac{\tau^{2}(x)}{c},
$$

where $c$ is the same parameter in Eq. 2.18. Assuming that the pumping test starts at time zero and position $x_{1}$, and the slope of drawdown is maximized at time $t_{\text {peak }}\left(x_{2}\right)$ and position $x_{2}$, a similar equation can be derived

$$
\sqrt{t_{\text {peak }}\left(x_{2}\right)}=\frac{1}{\sqrt{c}} \int_{x_{1}}^{x_{2}} \frac{d s}{\sqrt{D(s)}} .
$$

Figure 2.4 shows the diffusion process of an impulse source in a homogeneous medium, where the z-axis represents the transient pressure distribution at two 
different times. For a Heaviside source, the z-axis represents the slope distribution of transient pressure. Both diffusions propagate radially, and the value along the $\mathrm{z}$-axis in each figure reaches a maximum (with dark red color). By comparing times $t_{1}$ and $t_{2}$ (where $t_{1}<t_{2}$ ), the amplitude decreases as the time increases, which can be explained as the decay of wave propagation. In this thesis, we are interested in the wavefront with the maximum amplitude. Figure 2.5 shows the profile of these two diffusions at $t_{1}$ and $t_{2}$.

(a)

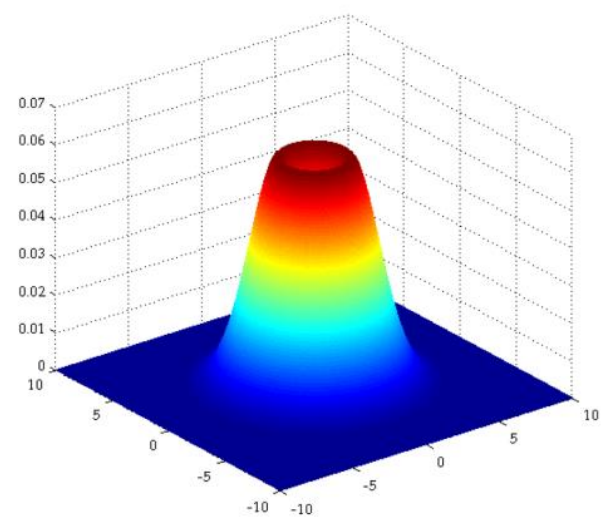

(b)

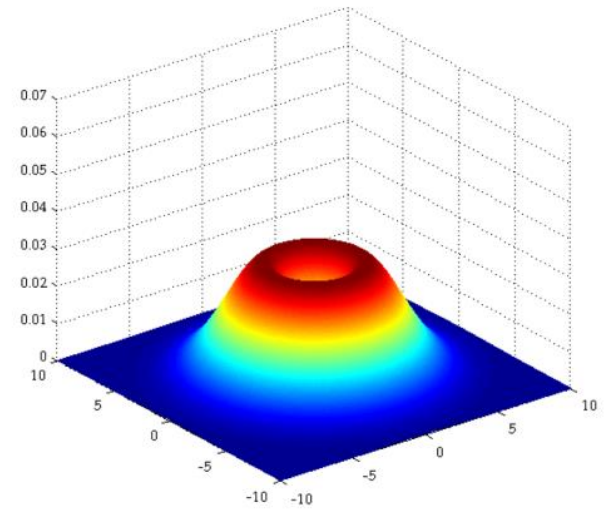

Figure 2.4. For an impulse source at the origin $(x=0, y=0)$, the $z$-axis represents the transient pressure. For a Heaviside source, the z-axis represents the slope of transient pressure, (a) at time $t_{1},(\mathbf{b})$ at time $t_{2}$, where $t_{2}>t_{1}$. 


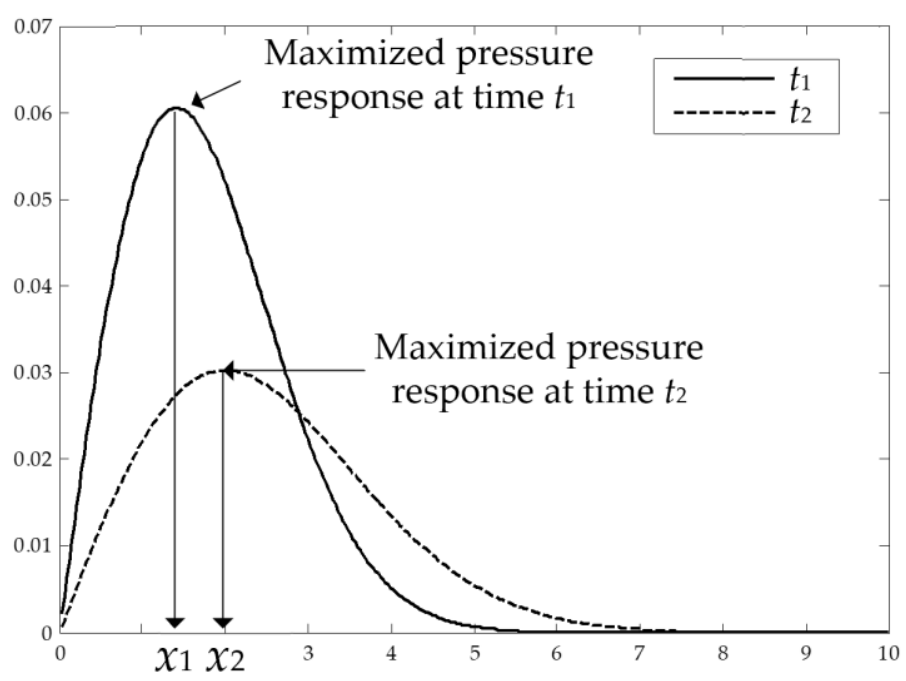

Figure 2.5. Profile of the pressure (for the impulse source) or slope of pressure (for the Heaviside source) in Figure $2.4(y=0, x \in[0,10])$, maximum z-axis values at $x_{1}$ and time $t_{1}$, then at $x_{2}$ and time $t_{2}$.

In a cross-well pumping test, $t_{\text {peak }}$ is the travel time which refers to the time when the slope of drawdown reaches a maximum. The concept of different travel times is generalized through the introduction of travel time diagnostic (Brauchler et al. 2003, Brauchler et al. 2011, Hu et al. 2011). As an example, the $t_{10}$ diagnostic is the time when the slope rises to $10 \%$ of its maximum amplitude. Following this definition, $t_{\text {peak }}$ is defined as $t_{100}$. Figure 2.6 shows three travel times: $t_{10}, t_{50}$, and $t_{100}$. 


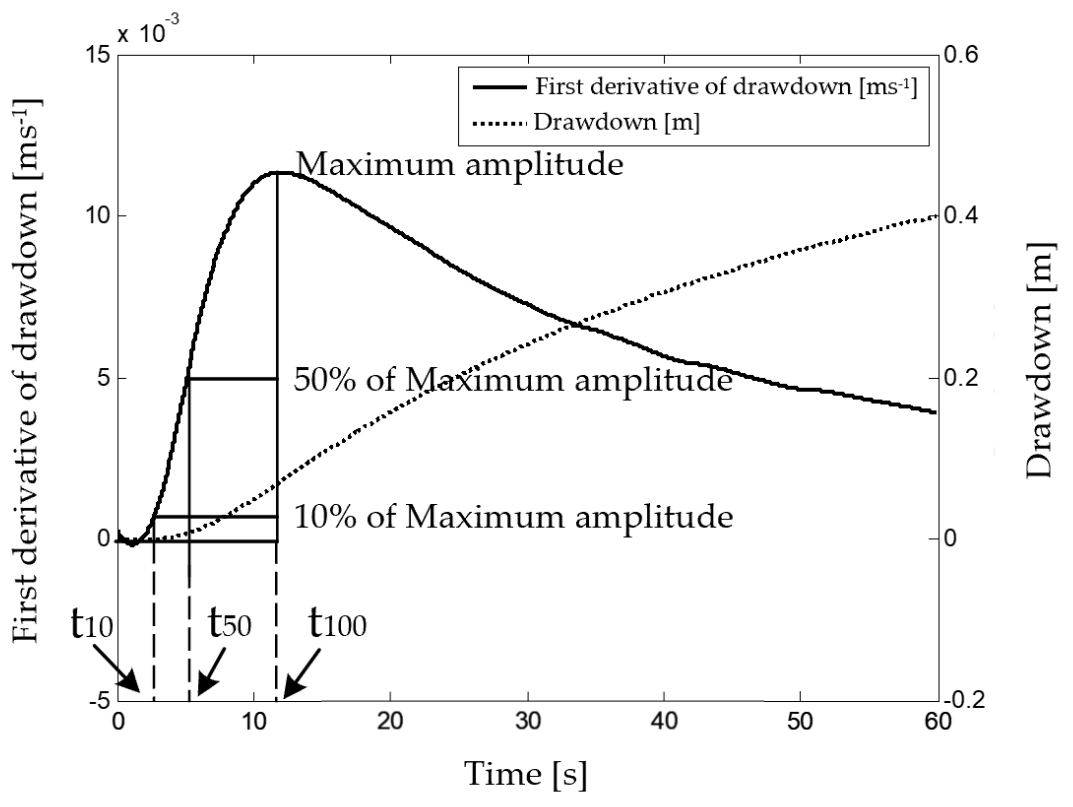

Figure 2.6. Three travel times $t_{10}, t_{50}$, and $t_{100}$ (Brauchler et al. 2003).

A transformation factor $f$ was introduced by Brauchler et al. (2003), and the relationship between travel time diagnostics and diffusivity can be described as

$$
\sqrt{t}=\frac{1}{\sqrt{c f}} \int_{x_{1}}^{x_{2}} \frac{d s}{\sqrt{D(s)}}
$$

where $t$ is the travel time diagnostic and $f$ is the transformation factor, which is a travel time diagnostic related to Lambert's $\mathrm{W}$ function and can be determined numerically. As this thesis focuses on the performance of the reconstruction algorithm, only $t_{100}$ is utilized for the inversion. For more information about the transformation factor, readers are referred to Brauchler et al. (2003) and Hu (2011).

The relationship between drawdown and diffusivity has been found, the next step is solving the eikonal equation. As Figure 2.3 shows, the ray trajectory indicates the propagation of a wavefront that is described by the eikonal equation, therefore the problem can transform to a determination of the ray trajectory based on the hydraulic diffusivity. Various numerical methods are available to 
determine ray trajectories (Brokešová 2006). Ray-tracing technique is used in this thesis and will be described thoroughly in subsection 2.2.3.

\subsubsection{Numerical discretization}

The investigation domain/profile $(\Omega)$ between a pumping well and an observation well is divided into a grid of $n$ small rectangular cells $\left(\Omega_{1}, \cdots, \Omega_{n}\right) . D_{j}$ is defined as the mean diffusivity in cell $\Omega_{j}$. Herein, the cells are distributed as a matrix, and the distribution resolution is defined as the number of rows multiplied by the number of columns. Therefore, the resolution is $4 \times 6=24$ and $n=$ 24 (Figure 2.7). A ray travels along a curve from the source through $\Omega$ to the receiver.

\begin{tabular}{|c|c|c|c|c|c|c|c|}
\hline \multirow[b]{2}{*}{$Z$} & $\Omega_{1}$ & $\Omega_{2}$ & $\Omega_{3}$ & $\Omega_{4}$ & $\Omega_{5}$ & $\Omega_{6}$ & \multirow[b]{2}{*}{ Receiver } \\
\hline & $\Omega_{7}$ & $\Omega_{8}$ & $\Omega_{9}$ & $\Omega_{10}$ & $\Omega_{1}$ & $\overrightarrow{\Omega_{12}} \longrightarrow$ & \\
\hline \multirow[t]{2}{*}{ Source } & $\Omega_{13}$ & $\Omega_{14}$ & $\Omega_{45}$ & $\Omega_{16}$ & $\Omega_{17}$ & $\Omega_{18}$ & \\
\hline & $\Omega_{19}$ & $\Omega_{20}$ & $\Omega_{21}$ & $\Omega_{22}$ & $\Omega_{23}$ & $\Omega_{24}$ & \\
\hline
\end{tabular}

Figure 2.7. Ray travels through a discrete area.

We assume that $m$ pumping tests are performed (i.e., $m$ different locations of sources or receivers), in other words, $m$ travel times can be sampled. By using the Ray-tracing technique, $m$ ray trajectories are determined. Let $s_{i j}$ be the length of the $i$ th ray trajectory in $\Omega_{j}$. If this $i$ th trajectory does not traverse $\Omega_{j}$, then $s_{i j}=0$.

Eq. 2.24 is thus rewritten as

$$
\sqrt{c t_{100}}=\sum_{j=1}^{n} \sqrt{\frac{1}{D_{j}}} s_{i j}, \quad i=1, \cdots, m .
$$

In matrix form, Eq. 2.26 becomes 


$$
\begin{gathered}
b=A x \\
b_{i}=\sqrt{c t_{100}}, \quad A_{i j}=s_{i j}, x_{j}=\sqrt{\frac{1}{D_{j}}} \\
i=1, \cdots, m, \quad j=1, \cdots, n,
\end{gathered}
$$

where $b$ is an $m$-vector consisting of $m$ observed travel times, and $A$ is an $m \times n$ matrix. The matrix element represents the trajectory length within each cell, and each row represents a ray trajectory. That is to say, $A$ records the ray paths in the investigation domain. $x$ is an $n$-vector, where each vector element is related to the diffusivity of each cell, and the vector $x$ shows the diffusivity distribution of the investigation domain.

\subsubsection{Ray-tracing technique}

The Fermat principle, also known as the principle of the shortest travel time path, asserts the path taken by a ray between two points is the path that can be traversed in the least amount of time. It is proven that the rays, defined by the eikonal equation, are identical to the curves defined by the Fermat principle (Lo and Inderwiesen 1994, Bruneau 2006, Born and Wolf 1999, Brokešová 2006). This principle describes various physical phenomena, e.g., reflection and refraction of light, the curve of fastest descent, and medical imaging using ultrasound.

Ray-tracing is a method to describe the ray trajectory numerically and has three main approaches: straight rays, ray bending, and network theory with

minimum-time paths. The first approach approximates every trajectory as a straight line. This approach is the fastest but is generally the least accurate, especially when the aquifer is heterogeneous. The ray bending approach adjusts ray paths by calculating local gradients. This method is fast and more accurate but 
may lead to a ray path with a local minimum time instead of a global minimum time. Network theory is based on a fixed grid of nodes. Every node can link to other nodes nearby through straight line segments. The trajectory from one node to another node is approximated using the connection of these line segments. All possible connections between source and receiver are considered and the trajectory with the minimum travel time will be chosen. This method takes the longest calculation time but guarantees a global minimum time (Jackson and Tweeton 1996). As it is more accurate, our experiments apply this approach to find the ray path of a hydraulic pressure signal.

Figure 2.8 show a grid that was proposed by Moser (1991). Figure 2.8a presents the distribution of nodes in one cell. Each cell has two nodes on every edge. Each node can only connect with other nodes that are in the same cell but not on the same edge. As Figure $2.8 \mathrm{~b}$ shows, the cells spanned by line segments form a network, so that a path from $S$ to $R$ can be approximated. The Dijkstra algorithm is widely used to find the shortest path in a network. The Dijkstra algorithm can be adapted by replacing distance with travel time. Further information about this algorithm is available from Dijkstra (1959) and Nakanishi and Yamaguchi (1986). Note that the accuracy of the network theory is strongly dependent on the density of nodes in a single cell and the density of cells in a grid (the reconstruction resolution). Accuracy can be improved by increasing either of the two densities, but at the cost of a more time-consuming computation. Hence, a small travel time path may not even be uniquely determined. For example, assuming that the investigation domain in Figure $2.8 \mathrm{~b}$ is homogeneous, and every line segment has equal weight, the orange path has the same travel time as the blue path. This problem would occur more often in heterogeneous cases, since each line segment could have a different weight. 
According to Snell's law, the rays are refracted at the interface of two materials due to different velocities in different materials. Herein, the rays travel along the lines spanned by limited and fixed nodes, they cannot follow the Snell's law at the interface of two cells with different diffusivities. However, both Snell's law and the ray-tracing technique follow Fermat principle. Therefore, the trajectories can better follow Snell's law if the nodes used in the ray-tracing technique are more densely distributed.

(a)

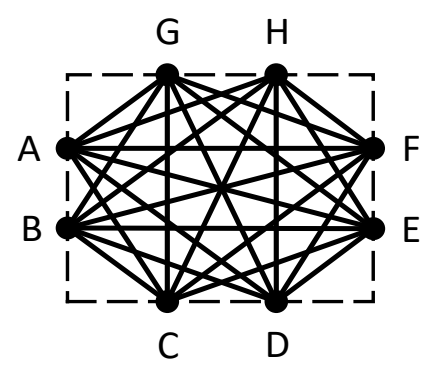

(b)

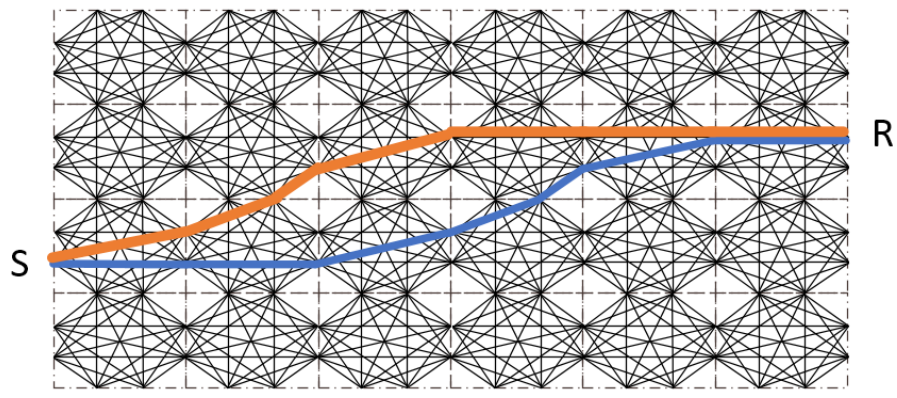

Figure 2.8. (a) Node distribution in a cell, (b) Two possible paths (orange and blue) with identical travel time from $S$ to $R$ in a network.

\subsubsection{Problem formulation}

The hydraulic problem is now transferred into a mathematical nonlinear problem after the discretization. The problem is described to find the solution of follow matrix equation

$$
b=A x,
$$

where the $b$ is a known vector, $A$ is an unknown matrix, and $x$ is the solution we want to approach.

In general, this equation is unsolvable if both $A$ and $x$ are unknown. However, signals preferentially penetrate cells with high diffusivity, since higher diffusivity leads to less travel time according to Eq. 2.24. By using ray-tracing technique, the 
ray trajectory can be determined by diffusivity distribution. In other words, $A$ is derived from $x$ in Eq. 2.28.

Note that, the relationship between $A$ and $x$ suggests that the Eq. 2.28 is not a linear problem and should be categorized as a nonlinear problem (Lo and Inderwiesen 1994). Two algorithms (i.e., SIRT and SIRT-Cimmino) are introduced to solve this nonlinear problem in the following subsections.

\subsubsection{SIRT algorithm}

Simultaneous Iterative Reconstruction Technique (SIRT) is a common implementation of Kaczmarz's method (Lo and Inderwiesen 1994), which is an iterative method for solving a system of linear equation and widely used in image processing and computed tomography. An introduction to Kaczmarz's method is provided in Appendix 7.6. The convergence of the iteration process, as well as the existence and uniqueness of the solution to this nonlinear problem have not been mathematically proven.

Given an $n$-vector $x$, the Euclidean norm of $x$ is denoted as $\|x\|=\sqrt{x^{T} x}$ and the $M$-norm of $x$ is denoted as $\|x\|_{M}=\sqrt{x^{T} M x}$, where $M$ is an $n \times n$-matrix. In this thesis, when discussing an iterative algorithm, the superscript with parenthesis stands for the number of iterations and the subscript stand for the position of

element, e.g., $x_{i}^{(k)}$ is the $i$ th element of $x$ in the $k$ th iteration, and $A_{i j}^{(k)}$ is the element in the $i$ th row and the $j$ th column of $A$ in the $k$ th iteration. $b$ is derived from observed travel time and is the only known variable in the following algorithm. We use $b^{o b s}$ instead of $b$ to distinguish other unknown variables.

The SIRT algorithm for travel time based hydraulic tomography can be introduced with the following steps (Figure 2.9): 
Step 1. Initialization. An initial vector $x^{\text {init }}$ is set. In the absence of prior information on diffusivity distribution, a homogeneous diffusivity distribution (i.e., all elements of $x^{\text {init }}$ are equal) is suggested. All signals thus travel along straight lines through the domain of interest.

Step 2. Utilization of ray-tracing technique. Ray-tracing is a tool to determine the path of signal propagation. This process reconstructs $A^{(k)}$ based on $x^{(k)}$,

$$
x^{(k)} \rightarrow A^{(k)}
$$

Step 3. Forward calculation with

$$
b^{(k)}=A^{(k)} x^{(k)}
$$

Step 4. Residual $\left(\Delta b^{(k)}\right)$ calculation with

$$
\Delta b^{(k)}=b^{o b s}-b^{(k)}
$$

Step 5. Criterion. Two criteria are feasible in this algorithm: either a fixed residual-dependent tolerance (e.g., $\left|\Delta b^{(k)}\right|<e$, where $e$ is a tolerance) or a fixed number of iterations. The algorithm performance at different iteration numbers is the focus of this study, so the second criterion is chosen.

Step 6. Incremental correction. To approach the solution, an incremental correction $\Delta x^{(k)}$ is embedded into each iteration with:

$$
\Delta x^{(k)}=W^{(k)} A^{(k)^{T}} N^{(k)} \Delta b^{(k)},
$$

where the diagonal matrix $W^{(k)}=\operatorname{diag}\left(1 / w_{1}, \cdots, 1 / w_{n}\right)$, and $w_{j}$ is the number of nonzero values in the $j$ th column of $A^{(k)}$; the diagonal matrix $N^{(k)}=$ $\operatorname{diag}\left(1 /\left\|a_{1}\right\|^{2}, \cdots, 1 /\left\|a_{m}\right\|^{2}\right)$, with $a_{i}$ representing the $i$ th row vector of $A^{(k)}$ and $\left\|a_{i}\right\|$ representing the Euclidean norm of $a_{i}$. Both $W^{(k)}$ and $N^{(k)}$ are derived from $A^{(k)}$ depending on the iteration step $k$. Hence, $W^{(k)}$ and $N^{(k)}$ are iteration dependent. 
Step 7. Iteration updates. The diffusivity vector $x$ is updated based on $x^{(k)}$ and incremental correction $\Delta x^{(k)}$ with:

$$
x^{(k+1)}=x^{(k)}+\Delta x^{(k)}
$$

The algorithm stops if $k+1$ meets the iteration step criterion in Step 1. Otherwise the calculation continues to the next iteration.

In this application, matrix $A$ is rebuilt every iteration with minimal influence on the algorithm convergence. Due to the specific iterative form in Eq. 2.32, two drawbacks could cause inversion failure: (a) if there is a cell $\Omega_{j}$ that is not traversed by any ray, then the $j$ th column of $A$ is a zero vector, $w_{j}=0$, and $W^{(k)}=$ $\operatorname{diag}\left(1 / w_{1}, \cdots, 1 / w_{n}\right)$ would not be generated since the denominator of $1 / w_{j}$ is zero; and (b) the result (diffusivity distribution) at one iteration step differs from the results at other iterations. In practice, the number of steps cannot be determined only by the residual convergence. Studies have shown that fewer iterations with a higher residual may possibly lead to better solutions and more iterations with a lower residual may lead to higher deviation (Brauchler et al. 2013a). Thus, a reliable and feasible rule to determine the optimal number of iteration steps and the selection of the best reconstruction is needed. 


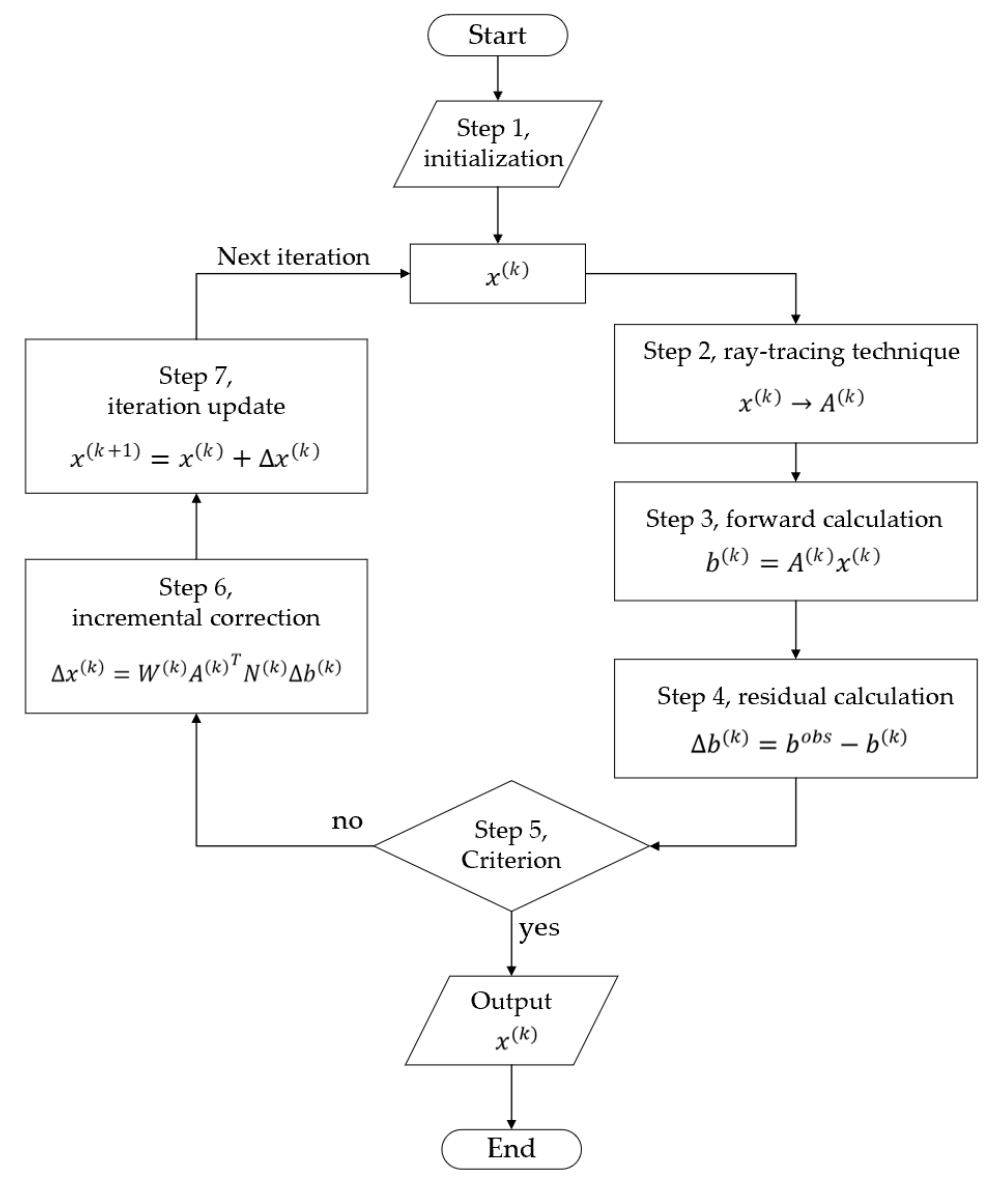

Figure 2.9. Flowchart of SIRT algorithm.

\subsubsection{SIRT-Cimmino algorithm}

In general, residuals show the convergence by applying SIRT to solve the nonlinear problem. However, even with the same degree of residuals, results with a different number of iteration steps (NIS) show large differences. In the literature, researchers estimate the optimal NIS empirically. Hence, a clearly defined result selection rule (or criterion) is required for the determination of NIS.

To overcome the drawbacks, we propose SIRT-Cimmino algorithm, which is based on SIRT but replaces the core iteration by Cimmino iteration. Figure 2.10 provides the overview of SIRT-Cimmino algorithm (Elble et al. 2010, Cimmino 1938). The Cimmino iteration modifies the incremental correction to avoid the case 
of division by zero (Eq. 2.32). An iterative dependent relaxation parameter is introduced to adjust the convergence velocity. Coupled with the new algorithm, a result selection rule is suggested. This rule is presented in the next chapter, and its feasibility will be proven through numerical tests.

Step 6 in SIRT is modified by following

Step 6. Incremental correction,

$$
\Delta x^{(k)}=\lambda_{k} A^{(k)^{T}} M^{(k)} \Delta b^{(k)},
$$

where matrix $M^{(k)}=\frac{1}{m} \operatorname{diag}\left(1 /\left\|a_{1}\right\|^{2}, \cdots, 1 /\left\|a_{m}\right\|^{2}\right)$, from which $a_{i}$ is the $i$ th row vector in $A^{(k)}, m$ is the number of rows in $A^{(k)}$, and $\lambda_{k}$ is the relaxation parameter defined below

$$
\lambda_{k}=\frac{\Delta b^{(k)^{T}} M^{(k)} \Delta b^{(k)}}{\left\|A^{(k)^{T}} M^{(k)} \Delta b^{(k)}\right\|_{2}^{2}} .
$$

The relaxation parameter in the iteration algorithm can be either a constant (iteration-independent) or a variable (iteration-dependent).

In $\mathrm{CT}, \mathrm{X}$-rays traverse human tissues and organs along straight lines, which implies that $A$ is a fixed matrix and iteration-independent. Thus, Eq. 2.28 becomes a linear problem and the SIRT-Cimmino converge to a solution $x^{*}$ of $\min \| A x-$ $b \|_{M}$, if $\lambda \in\left(0,2 / \sigma^{2}\right)$, where $\sigma$ denotes the maximum singular value of $A^{T} M A$ (Elfving et al. 2010). The Matrix $A$ is rebuilt in each iteration and varies while the diffusivity field is regenerated by incremental correction. Therefore, it is reasonable to define an $A$-dependent $\lambda$ considering convergence. Santos (1987) proposed the construction of $\lambda$ in Eq. 2.35, which minimized $\left\|x^{*}-x^{k}\right\|$ in each iteration, where $x^{*}$ is a solution of $A x=b$ (Elfving et al. 2010). 


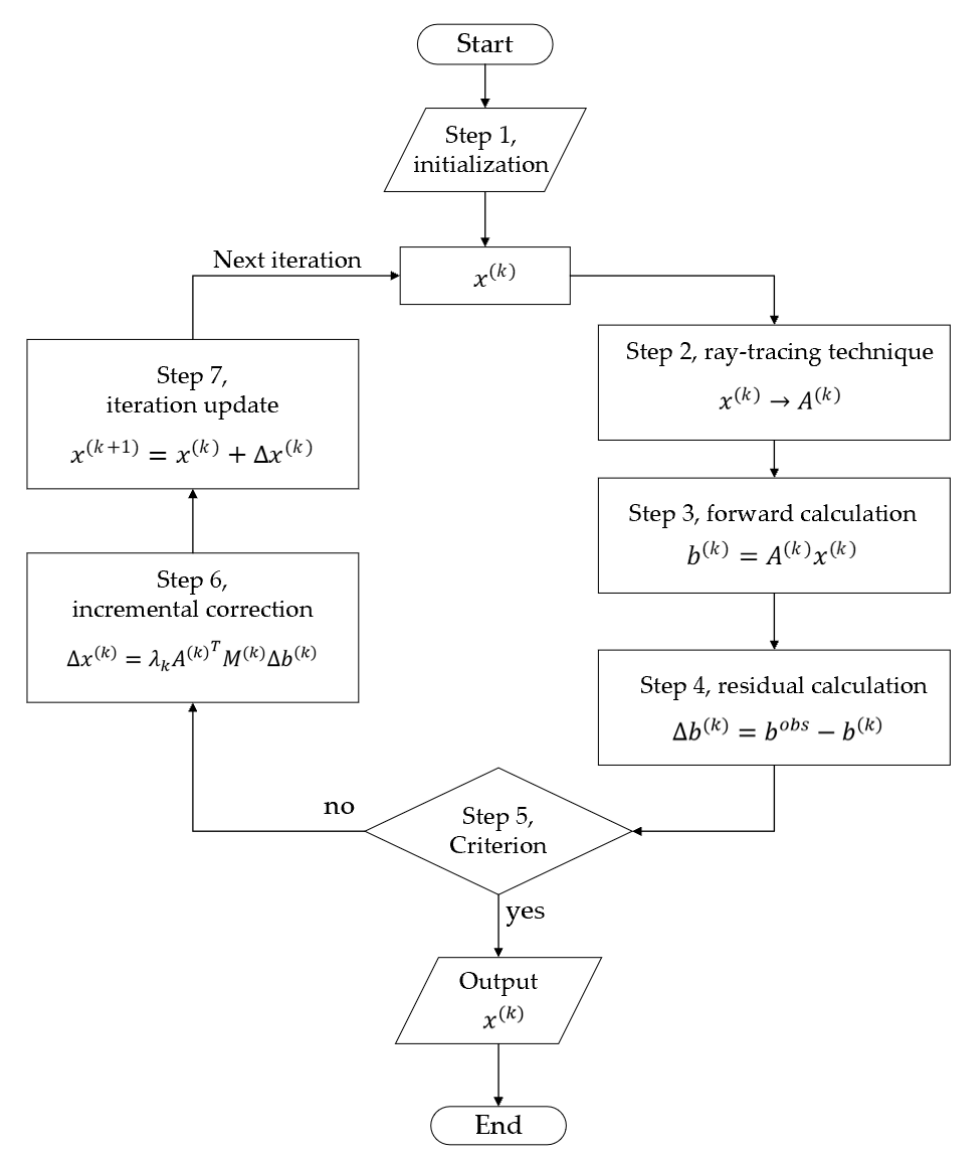

Figure 2.10. Flowchart of SIRT-Cimmino algorithm.

\subsubsection{Residual and criterion}

Residuals describe the difference between observed travel times and approximated travel times from ray-tracing in each iteration. The definition is given by:

$$
R=\frac{\sqrt{\sum_{i=1}^{m}\left(\sqrt{t_{i}^{(k)}}-\sqrt{t_{i}}\right)^{2}}}{\sum \sqrt{t_{i}}}
$$

where $t_{i}^{(k)}$ denotes the approximated travel time of the $i$ th signal in the $k$ th iteration, and $t_{i}$ denotes the observed travel times of the $i$ th signal (Brauchler et al. 2003, Hu et al. 2011). 


\section{Numerical Studies}

The feasibility verification of SIRT-Cimmino algorithm takes place first by numerical studies. Three numerical aquifer models with predefined diffusivity distributions ("truth distributions") are presented in this chapter. Flow characterization is based on the equivalent porous medium approach.

\subsection{Layered Aquifer Model}

\subsubsection{Predefined diffusivity distribution and model setup}

A 2D axisymmetric numerical groundwater flow model was built using the finite element software COMSOL Multiphysics ${ }^{\circledR}$ to simulate a series of cross-well multi-level short-term pumping tests with transient flow conditions. The model consists of three parts: the area of interest for parameter estimation $(4 \mathrm{~m} \times 3.2 \mathrm{~m}$, blue zone in Figure 3.1), the homogeneous zone $(10 \mathrm{~m} \times 3.2 \mathrm{~m}$, dark grey in Figure $3.1)$, and the homogeneous surrounding domain $(24 \mathrm{~m} \times 30 \mathrm{~m}$, light grey in Figure 3.1).

For convenience, high-diffusivity and low-diffusivity are notated by high- $D$ and low- $D$, respectively. This model is provided with an inclined high- $D$ band (Figure 3.2), the remaining background low-D zone is homogenous. Regarding the possible range of hydraulic parameters of the fluvio-sedimentary aquifer, the diffusivity value is set to $10 \mathrm{~m}^{2} \mathrm{~s}^{-1}$ within the high- $D$ area (green band in Figure 3.2) and $0.2 \mathrm{~m}^{2} \mathrm{~s}^{-1}$ within the low-D zone (blue zone in Figure 3.2), based on earlier studies (Hu et al. 2011, Bayer and Finkel 2004). The diffusivity contrast ratio is 50.

To eliminate the influence of boundary effects during the simulation, an infinite element domain with a scaling factor of 1000 is added to the right of the study zone with constant head (Figure 3.1, with only the first $30 \mathrm{~m}$ shown). In this domain, rational coordinate scaling is utilized to stretch the finite element domain 
where the dependent variables vary less with radial distance. The surrounding area is designated as a homogenous isotropic material, to maintain the continuity of diffusivity at the boundary of the area of interest. According to the measured value of hydraulic parameters in a fluvio-sedimentary aquifer by $\mathrm{Hu}(\mathrm{Hu} 2011)$, the hydraulic conductivity, specific storage and porosity are set as $K=8 \times 10^{-5} \mathrm{~ms}^{-}$ ${ }^{1}, S_{S}=4 \times 10^{-4} \mathrm{~m}^{-1}$, and $0.2(-)$, respectively. The diffusivity $D$ is therefore set as 0.2 $\mathrm{m}^{2} \mathrm{~s}^{-1}$, since $D=K S_{s}^{-1}$.

Each simulated pumping test had a pumping duration of ten minutes, and the head sampling interval is $0.02 \mathrm{~s}$. The area of interest size and the pumping and observation positions are shown in Figure 3.2. Eight pumping and eight observation positions are represented by $\mathrm{S} 1, \ldots, \mathrm{S} 8$ and $\mathrm{R} 1, \ldots, \mathrm{R} 8$, respectively. The radius of the pumping well and observation well is $0.05 \mathrm{~m}$. The initial head of the aquifer is set to $10 \mathrm{~m}$. The pumping tests are simulated sequentially at each pumping position while the head changes are recorded at all eight observation points throughout each test. This resulted in $8 \times 8$ drawdown data sets. As an example, Figure 3.3 shows the head data recorded at the eight observation points when the pumping test is performed at S1. During each simulated pumping test, the constant head boundary is not reached.

Figure 3.4 shows the travel time of the signal is sent $\mathrm{S} 1$ and is captured at R3. The pressure signal travel time is derived by applying first order differentiation to the recorded drawdown curves. The inversion is implemented with software TomoGo v1.06 (Qiu et al. 2019). A detail description of TomoGo is available in Appendix 7.3.

The resolution is mainly determined on the number of signals. A reconstruction under high resolution but with less non-uniqueness and uncertainty is an aim of the inversion. Three kinds of resolutions are considered in our experiments. 


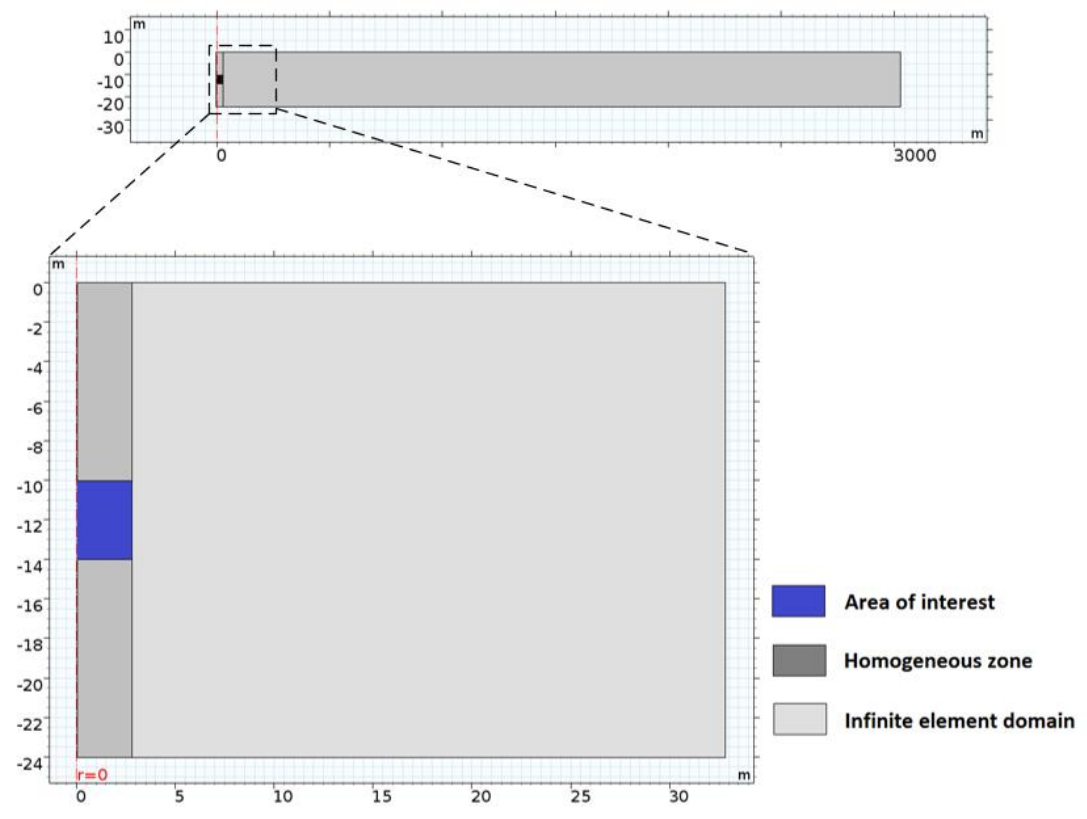

Figure 3.1. The geometry of the 2D axisymmetric model.

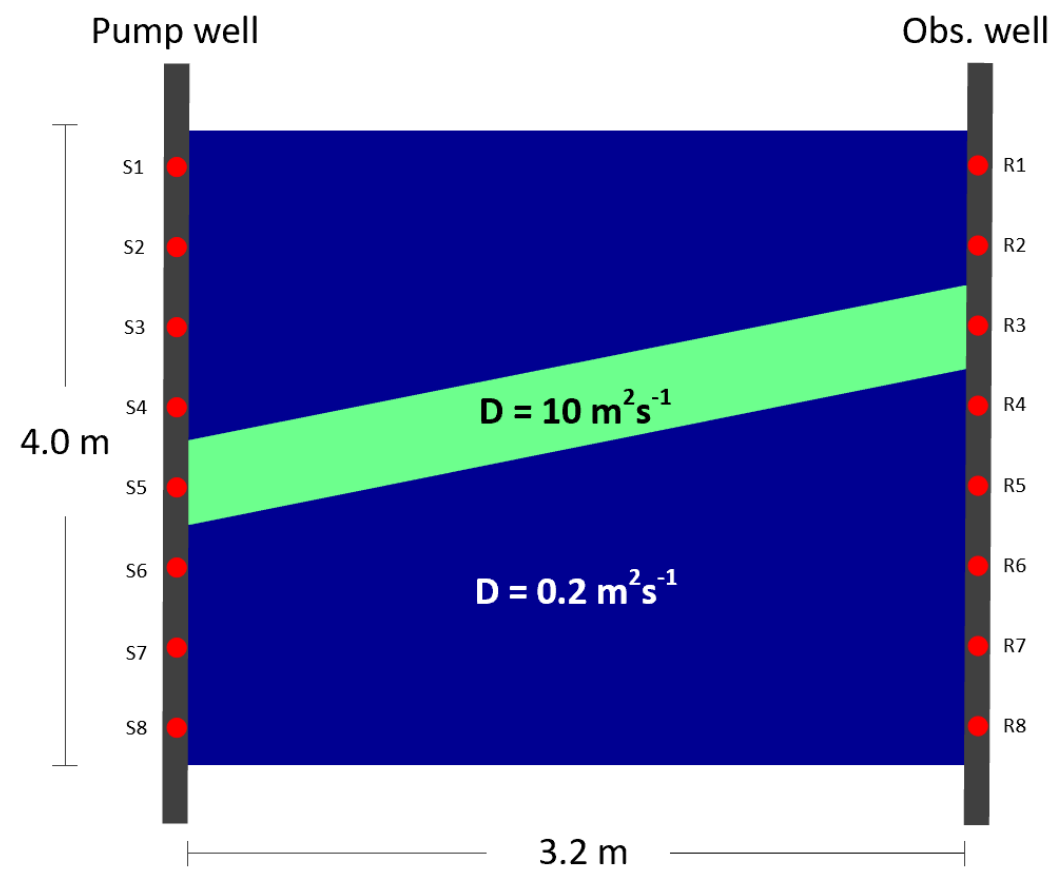

Figure 3.2. Predefined diffusivity distribution. 


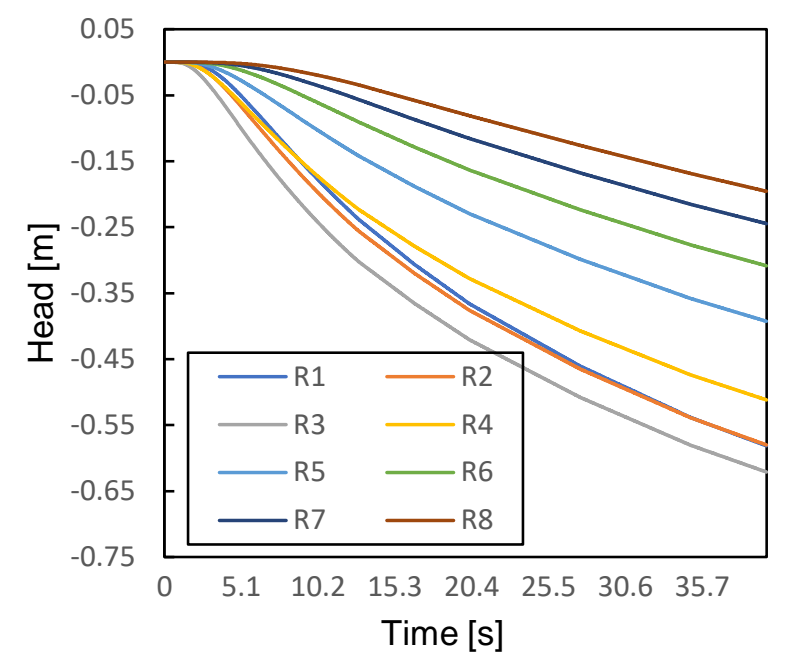

Figure 3.3. Head drawdown recorded at R1-R8 while pumping at S1.

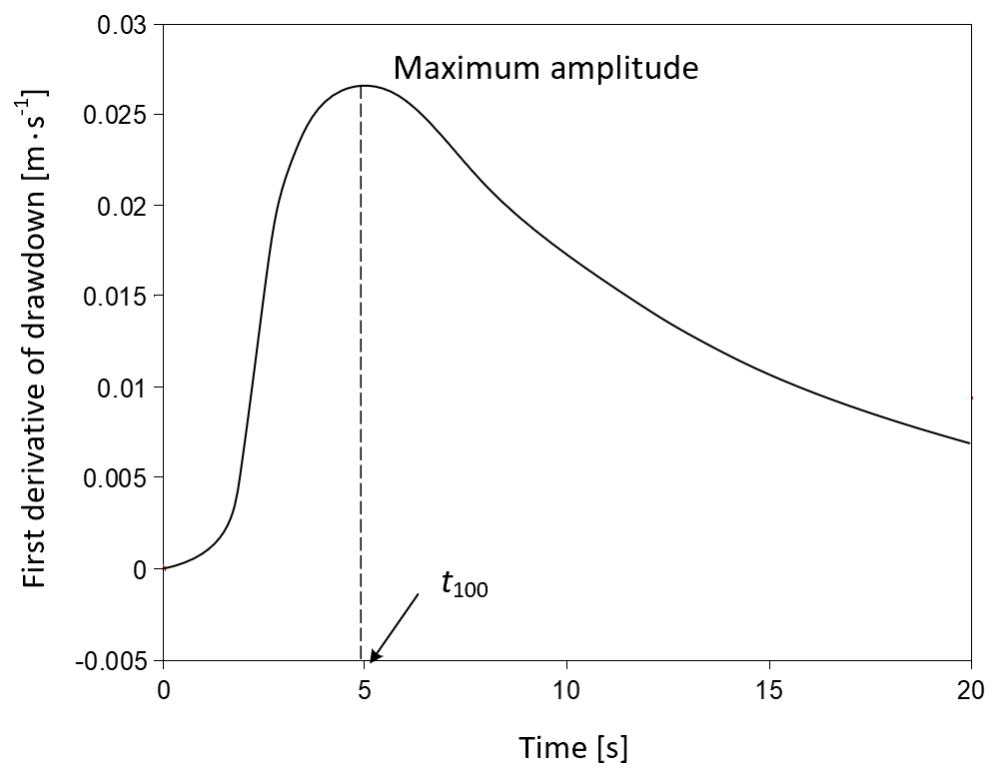

Figure 3.4. First derivative of drawdown recorded at R3, and the correspondent $t_{100}$ while pumping at $S 1$.

\subsubsection{Result selection rule for SIRT}

In each iteration, a diffusivity tomogram is reconstructed, and it differs from that in different iteration. Therefore, the number of iteration steps (NIS) is a key 
parameter for the reconstruction process, and a rule to determine an appropriate NIS must be defined and verified.

As NIS increases, the residuals in SIRT are reduced and converge to a nonzero constant (Figure 3.5). Due to the complex of non-linearity, a standard NIS cannot be determined mathematically. Brauchler et al. (2003) empirically utilized an NIS of 10, and $\mathrm{Hu}(2011)$ utilized an NIS of 8.

For the inversion constraint, we first assume that the investigation domain is homogeneous, and every trajectory in this domain is a straight line. By using the above-mentioned straight ray inversion approach as the first inversion step, a vector with uniform diffusivity value is obtained. This diffusivity value is set as the average value of the elements in the vector, i.e., the initial value of diffusivity for the heterogeneous domain in the following inversion steps. In our case, a range for diffusivity during the inversion calculation is set with lower and upper limits defined as 0.01 times and 100 times the initial diffusivity, respectively. In the model, the initial diffusivity is set as $0.78 \mathrm{~m}^{2} \mathrm{~s}^{-1}$, the lower and upper limits are $0.0078 \mathrm{~m}^{2} \mathrm{~s}^{-1}$ and $78 \mathrm{~m}^{2} \mathrm{~s}^{-1}$, respectively.

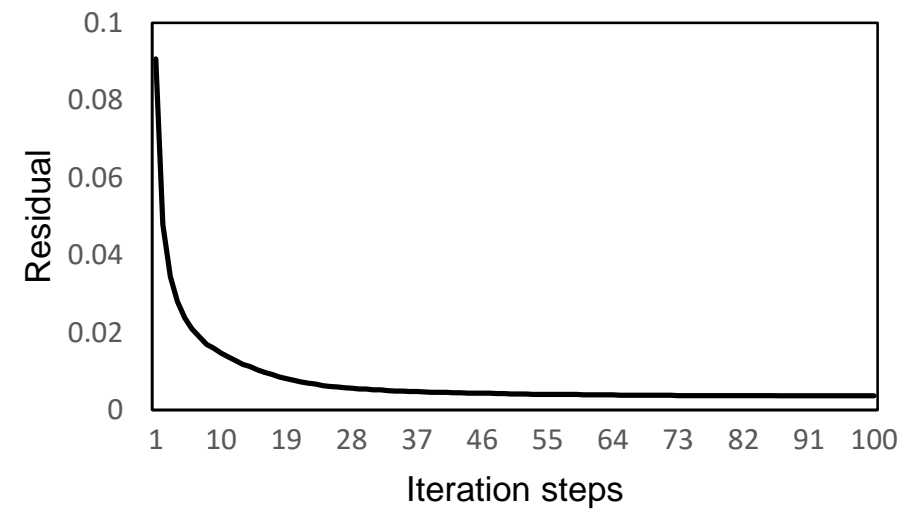

Figure 3.5. Residual for 100 iteration steps by using SIRT under $8 \times 8$ resolution in the model. 
The inversion results at the 10th, 25th, and 100th steps are shown in Figure $3.6 \mathrm{~b}-\mathrm{d}$. Each of these three distributions indicates the existence of a high- $D$ zone connecting S5 and R3. Comparison with the predefined distribution (Figure 3.6a) shows that the diffusivity values in the high- $D$ zones are lower than 10 . Diffusivity in the high- $D$ zone increases as NIS increases, but is still too low in the center after 100 iterations, despite convergence of the residual after 25 iteration steps. After 25 steps, the diffusivity near S5 and R3 increases so rapidly that it reached the upper limit $\left(78.49 \mathrm{~m}^{2} \mathrm{~s}^{-1}\right)$. This is in agreement with the findings by Brauchler et al. (2003), who mentioned that a deviation may occur with a large NIS. Mathematically, the solution non-uniqueness is a possible explanation, and SIRT might approach to a wrong solution. After comparison between the inversion result at every iteration step (within 100 steps) and the "true" distribution in our case, the result at the 10th step is considered the best reconstruction, and 10 is considered an appropriate value for NIS. Similarly, the optimal NIS for the model with different inversion resolutions is determined (Table 3.1). 
(a)

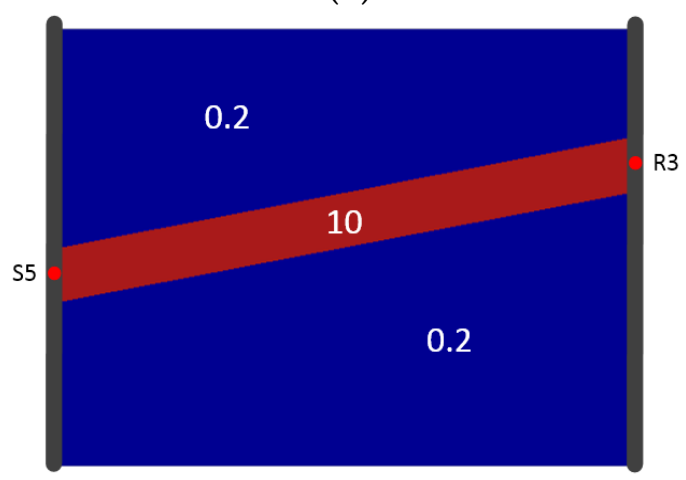

(c)

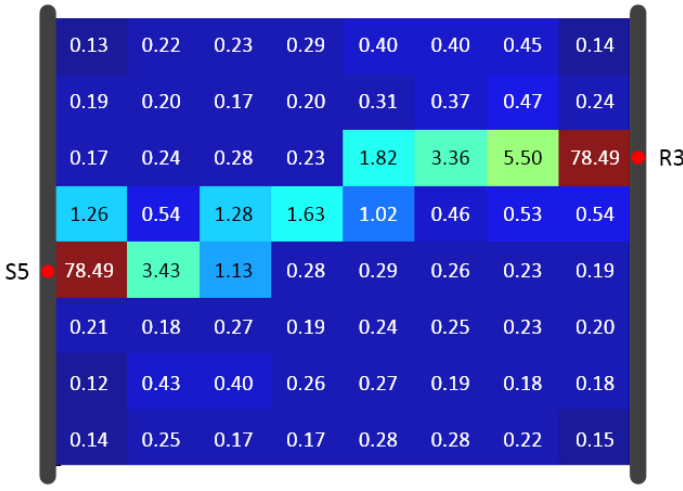

(b)

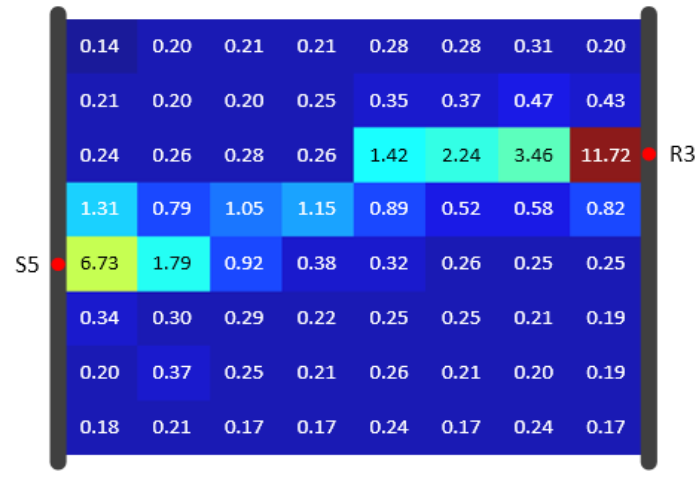

(d)

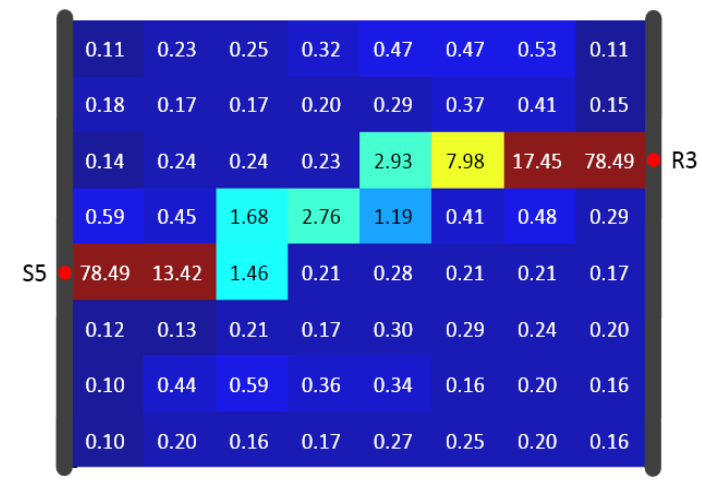

Figure 3.6. Diffusivity $\left[\mathrm{m}^{2} \mathrm{~s}^{-1}\right]$ tomograms based on the inversion of $t_{100}$ and SIRT under $8 \times 8$ resolution with different numbers of iterations. (a) Predefined diffusivity ("truth") distribution; and (b-d) inversion results after 10, 25, and 100 iterations, respectively.

Table 3.1. Optimal number of iteration steps for using SIRT under three different model resolutions.

\begin{tabular}{cccc}
\hline & \multicolumn{3}{c}{ SIRT } \\
\cline { 2 - 4 } & $\mathbf{8} \times \mathbf{6}$ & $\mathbf{8} \times \mathbf{8}$ & $\mathbf{1 2} \times \mathbf{1 2}$ \\
\hline$t_{100}$ & 11 & 10 & 5 \\
\hline
\end{tabular}

As shown in Table 3.1, the optimal NIS when using SIRT is dependent on the model resolution. The influence of the travel time type (e.g., $t_{100}, t_{50}$ and $\left.t_{10}\right)$ is not investigated in this work. In practice, when the prior information on hydraulic parameters within the investigated area is insufficient, it is hard for SIRT users to determine the optimal NIS and subsequently obtain the best result. 


\subsubsection{Result selection rule for SIRT-Cimmino}

Figure 3.7 shows the residuals after 50 iteration steps when using SIRTCimmino in the Model. Oscillation is found and the convergence is not easily determined. Mathematically, this behavior can be explained by the rebuilding of matrix $A$ at each iteration and the non-uniqueness of the solution. Rebuilding matrix $A$ disturbs the residual convergence and even leads to a separate solution.

As shown in Figure 3.5, the residual is stabilized after several steps, as the SIRT algorithm trends toward a solution. This means that the residual values belong to a single solution. The divergent behavior in Figure 3.7 therefore indicates several solution approaches, which are represented by subsequences. For instance, the green subsequence in the Figure indicates a possible solution approach.

The selection of a result for SIRT-Cimmino is proposed through the following steps:

(1). Calculating 50 steps of iteration (due to computational time);

(2). Selecting a convergent subsequence with a low residual if convergent subsequences exist;

(3). Choosing the step with the lowest residual in this convergent subsequence as the optimal NIS and the corresponding result as the SIRT-Cimmino reconstruction.

In Figure 3.7, the step marked with black diamond is chosen as the optimal iteration step. 


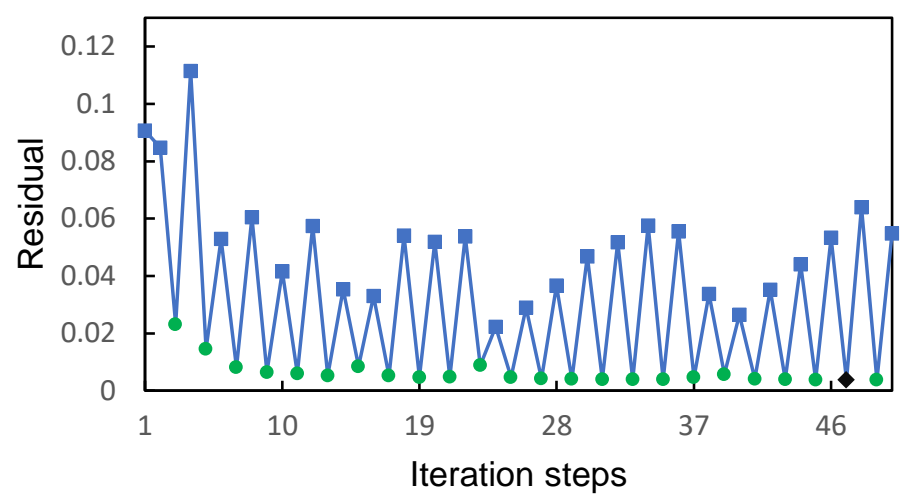

Figure 3.7. Residual of travel time for 50 iteration steps by using $t_{100}$ and SIRTCimmino under $8 \times 8$ resolution in the model.

\subsubsection{Reconstruction comparison of SIRT and SIRT-Cimmino of the model}

Both algorithms use $t_{100}$ data for reconstruction of the diffusivity distribution. Figure 3.9-17 show the inversion results with resolutions of $8 \times 6,8 \times 8$, and $12 \times 12$ (using the same color scale). In each SIRT result (Figure 3.9a, 16a, and 17a), the values in the high- $D$ zones are nearly one. These values do not clearly distinguish the high- $D$ zone from the background. In comparison, each SIRT-Cimmino result shows a clear high-D zone with better connectivity.

RMSE and the correlation coefficient are calculated and listed in Table 3.2. The comparison shows that both algorithms have similar RMSE values. The SIRTCimmino has better performance with respect to the correlation coefficient. That is to say, the SIRT-Cimmino result delivers a higher similarity to the predefined distribution. In addition, the correlation coefficient increases as resolution increases, since the higher resolution improves the description of the high- $D$ zone (the main structural feature). In other words, the correlation coefficient is extremely sensitive to this zone. 


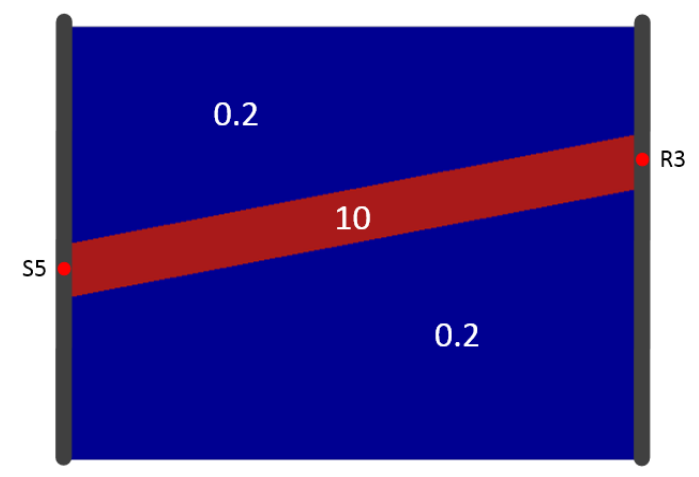

Figure 3.8. Predefined diffusivity ("truth") $\left[\mathrm{m}^{2} \mathrm{~s}^{-1}\right]$ distribution of the model.

(a)

\begin{tabular}{|cccccc}
0.15 & 0.22 & 0.22 & 0.29 & 0.25 & 0.22 \\
0.20 & 0.17 & 0.22 & 0.33 & 0.43 & 0.41 \\
\hline 0.24 & 0.26 & 0.27 & 1.41 & 2.96 & 13.11 \\
\hline S5 R3 \\
\hline 1.17 & 0.96 & 1.09 & 0.82 & 0.47 & 0.81 \\
\hline 5.34 & 1.28 & 0.37 & 0.29 & 0.23 & 0.24 \\
\hline 0.32 & 0.46 & 0.25 & 0.25 & 0.22 & 0.20 \\
0.20 & 0.34 & 0.23 & 0.24 & 0.19 & 0.19 \\
0.18 & 0.19 & 0.17 & 0.25 & 0.24 & 0.18
\end{tabular}

(b)

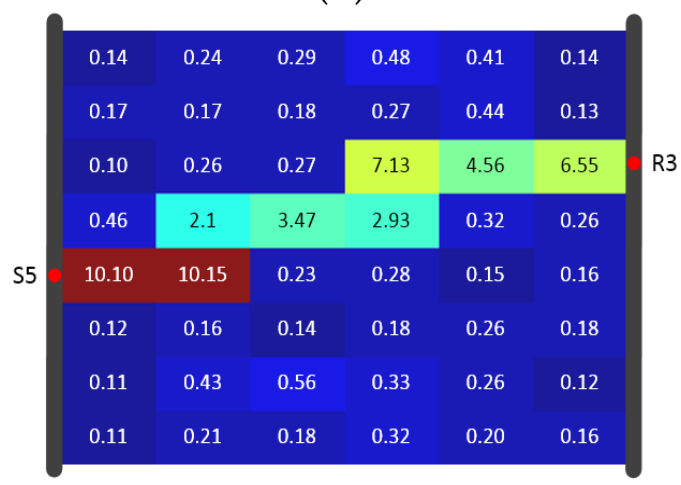

Figure 3.9. Algorithm result comparison for the model under $8 \times 6$ resolution of the (a) SIRT result and (b) SIRT-Cimmino result, shown in diffusivity $\left[\mathrm{m}^{2} \mathrm{~s}^{-1}\right]$.

(a)

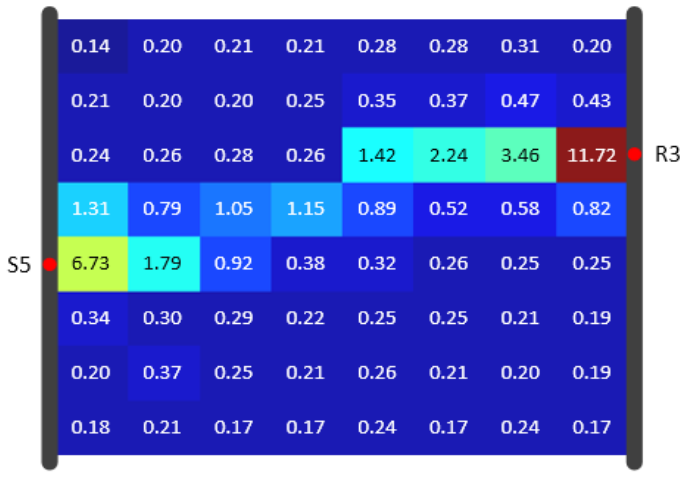

(b)

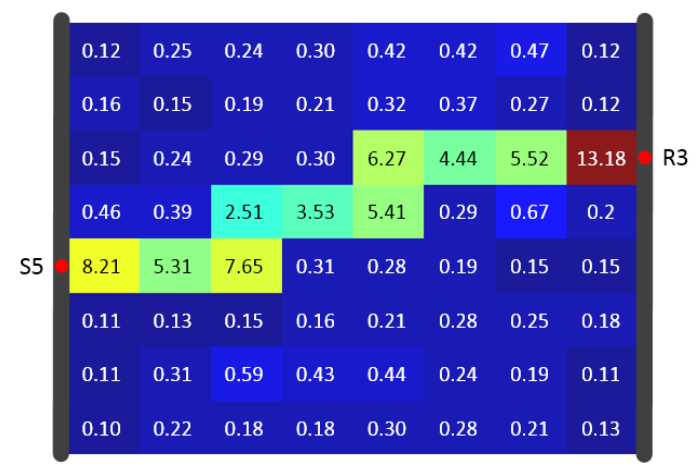

Figure 3.10. Algorithm result comparison for the model under $8 \times 8$ resolution of the (a) SIRT result and (b) SIRT-Cimmino result, shown in diffusivity $\left[\mathrm{m}^{2} \mathrm{~s}^{-1}\right]$. 
(a)

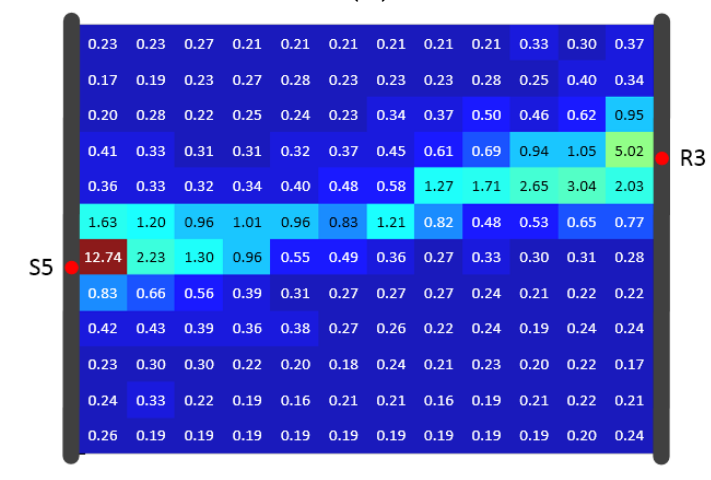

(b)

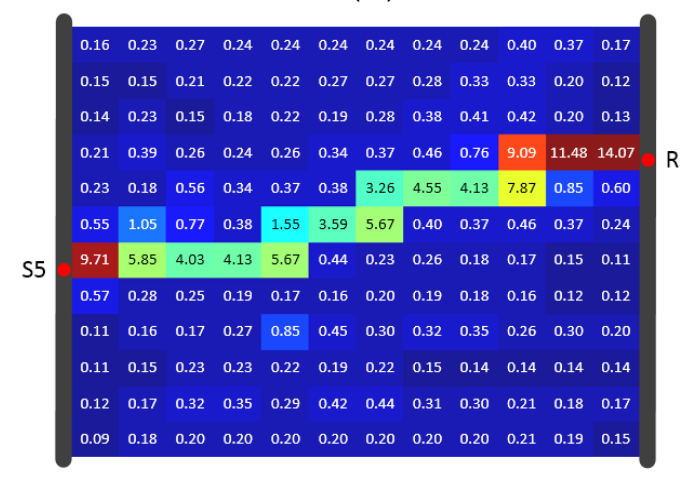

Figure 3.11. Algorithm result comparison for the model under $12 \times 12$ resolution of the (a) SIRT result and (b) SIRT-Cimmino result, shown in diffusivity $\left[\mathrm{m}^{2} \mathrm{~s}^{-1}\right]$.

(a)

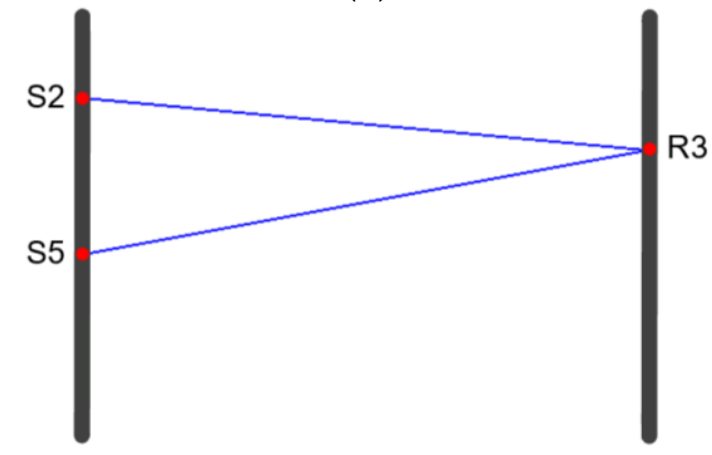

(c)

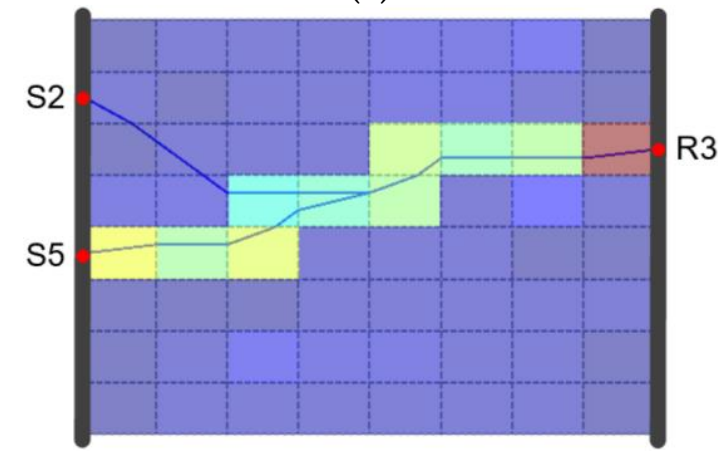

(b)

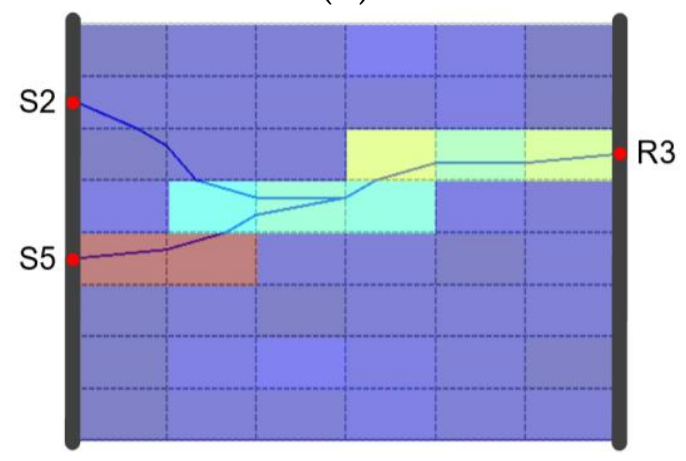

(d)

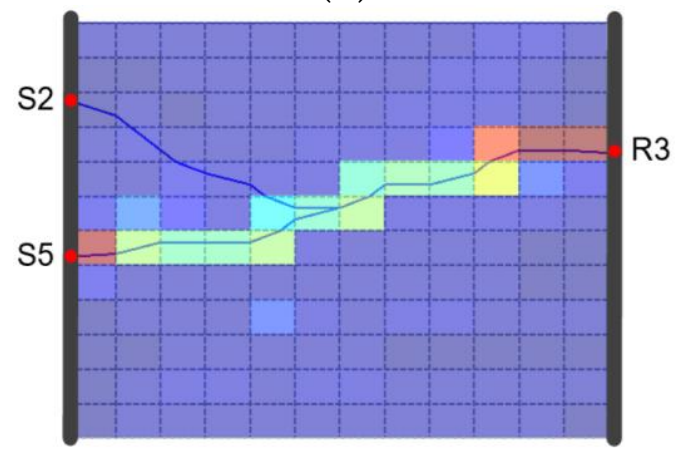

Figure 3.12. The trajectories of ray S2R3 and S5R3 based on the inversion of $t_{100}$ and SIRT-Cimmino under different resolutions. (a) The ray trajectories in homogeneous aquifer, (b) the ray trajectories in $8 \times 6$ resolution, (c) the ray trajectories in $8 \times 8$ resolution, $(\mathbf{d})$ the ray trajectories in $12 \times 12$ resolution. 
Table 3.2. RMSE (Root Mean Square Errors) and correlation coefficient of the model inversion using SIRT and SIRT-Cimmino.

\begin{tabular}{ccccccc}
\hline & \multicolumn{3}{c}{ RMSE } & \multicolumn{3}{c}{ Correlation Coefficient } \\
\cline { 2 - 7 } & $8 \times 6$ & $8 \times 8$ & $12 \times 12$ & $8 \times 6$ & $8 \times 8$ & $12 \times 12$ \\
\hline SIRT & 4.04 & 3.55 & 6.41 & 0.70 & 0.70 & 0.77 \\
\hline SIRT-Cimmino & 2.86 & 3.77 & 4.24 & 0.73 & 0.72 & 0.79 \\
\hline
\end{tabular}

\subsection{Laid Y-shape Aquifer Model}

\subsubsection{Predefined diffusivity distribution and model setup}

The performance of inversion algorithms is evaluated in a laid Y-shape model. We built this model with the same setup to the layered model except the predefined diffusivity distribution within the area of interest. This model has a lying Y-shaped high-diffusivity zone (Figure 3.13). The diffusivities in the high- $D$ and low- $D$ zone are $10 \mathrm{~m}^{2} \mathrm{~s}^{-1}$ and $0.2 \mathrm{~m}^{2} \mathrm{~s}^{-1}$, respectively.

Similar to previous processing on the layered model, a series of cross-well multi-level pumping tests are simulated, travel time are derived, NIS for using SIRT under different resolution are estimated and shown in Table 3.3. 


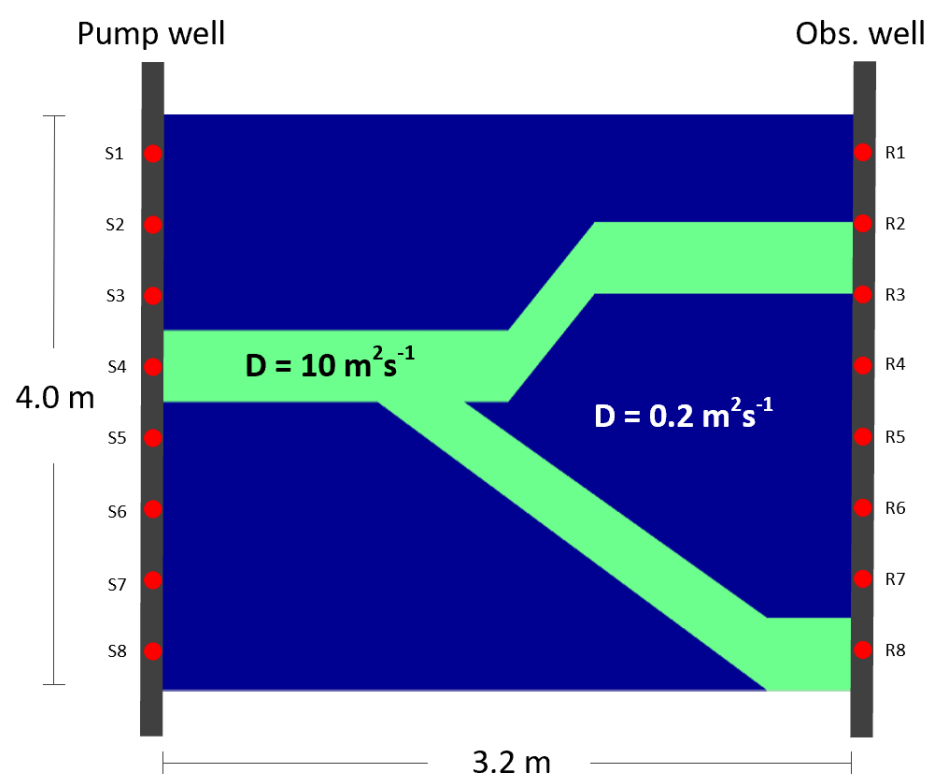

Figure 3.13. Predefined diffusivity distribution.

Table 3.3. Optimal number of iteration steps for using SIRT under three different model resolutions.

\begin{tabular}{cccc}
\hline & \multicolumn{3}{c}{ SIRT } \\
\cline { 2 - 4 } & $8 \times 6$ & $8 \times 8$ & $12 \times 12$ \\
\hline$t_{100}$ & 5 & 5 & 3 \\
\hline
\end{tabular}

\subsubsection{Reconstruction comparison of SIRT and SIRT-Cimmino of the model}

Both algorithms use $t_{100}$ data for reconstruction of the diffusivity distribution given in Figure 3.14. Figure 3.15-22 show the inversion results with resolutions of $8 \times 6,8 \times 8$, and $12 \times 12$ (using the same color scale), respectively. Comparison with the "true" diffusivity distribution (Figure 3.14) reveals that the lying Y-shaped high-D zone could be reconstructed. The reconstructions of SIRT are generally worse than the reconstructions from SIRT-Cimmino. This visual assessment coincides with the correlation coefficient calculation, since the correlation coefficient of SIRT-Cimmino is overall larger than that of SIRT, especially at the 12 $\times 12$ resolution (Table 3.4). 
The RMSE calculation does not show any advantage for SIRT-Cimmino in either Model A or Model B. There are two possible reasons. First, the discretization method assumes that the research area is divided into rectangles, which cannot approximate the inclined edge (shape) of the high- $D$ zone perfectly. Second, the high values near R8 in SIRT-Cimmino influence the overall RMSE.

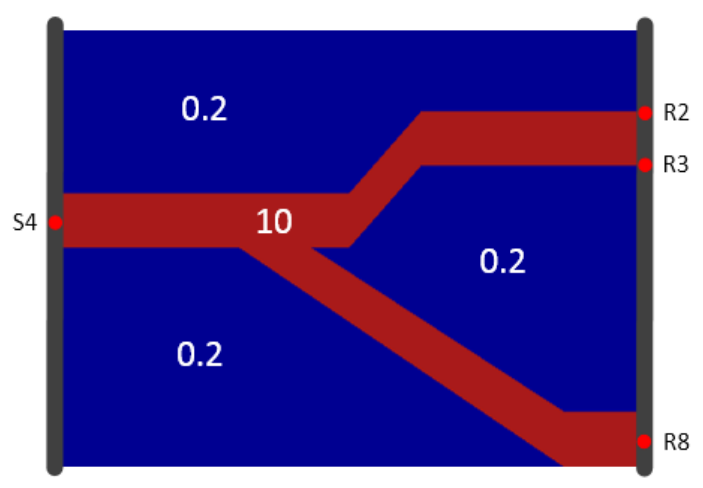

Figure 3.14. Predefined diffusivity distribution $\left[\mathrm{m}^{2} / \mathrm{s}\right]$ of the model.

(a)

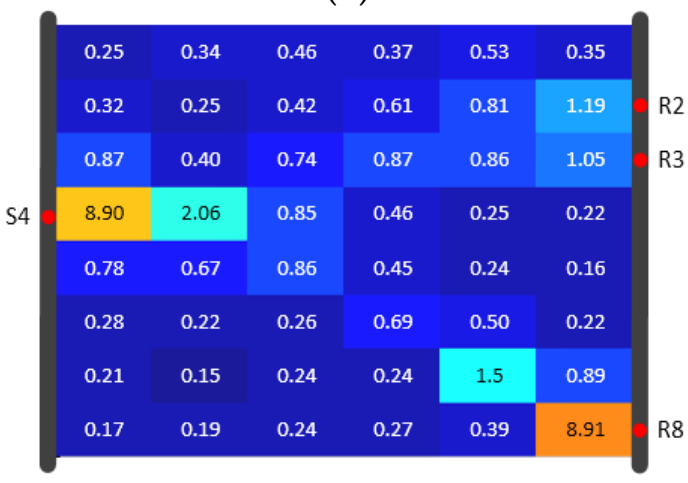

(b)

\begin{tabular}{|c|c|c|c|c|c|}
\hline 0.12 & 0.39 & 0.68 & 0.42 & 0.53 & 0.04 \\
\hline 0.07 & 0.18 & 0.28 & 0.59 & 0.97 & 7.48 \\
\hline 0.19 & 0.84 & 4.68 & 3.55 & 3.55 & 4.37 \\
\hline 2.83 & 1.88 & 2.14 & 0.20 & 0.20 & 0.03 \\
\hline 0.24 & 0.25 & 4.31 & 1.02 & 0.16 & 0.10 \\
\hline 0.10 & 0.21 & 0.17 & 6.53 & 0.93 & 0.09 \\
\hline 0.10 & 0.12 & 0.20 & 0.33 & 12.2 & 0.20 \\
\hline 0.12 & 0.21 & 0.32 & 0.29 & 0.51 & 17.14 \\
\hline
\end{tabular}

Figure 3.15. Algorithm result comparison for the model under $8 \times 6$ resolution of the (a) SIRT result and (b) SIRT-Cimmino result, shown in diffusivity $\left[\mathrm{m}^{2} \mathrm{~s}^{-1}\right]$. 
(a)

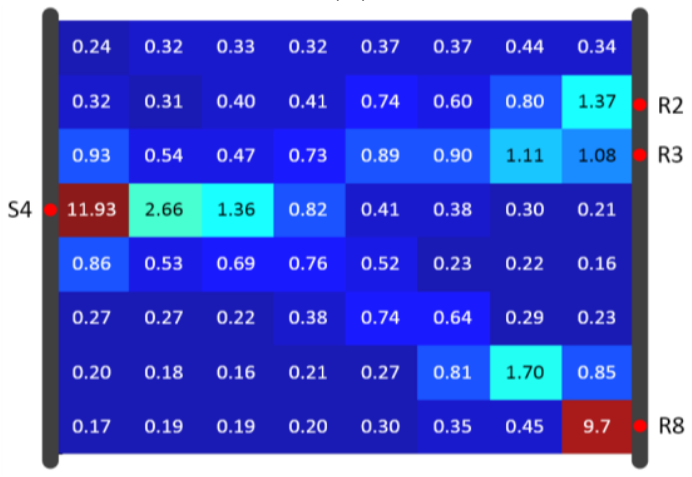

(b)

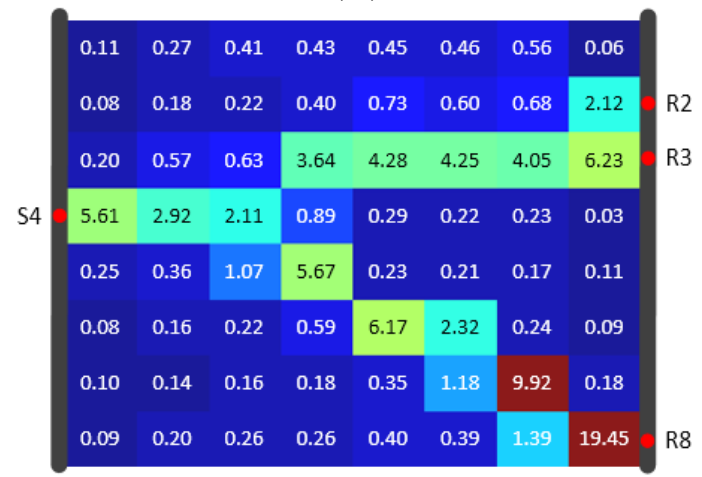

Figure 3.16. Algorithm result comparison for the model under $8 \times 8$ resolution of the (a) SIRT result and (b) SIRT-Cimmino result, shown in diffusivity $\left[\mathrm{m}^{2} \mathrm{~s}^{-1}\right]$.

(a)

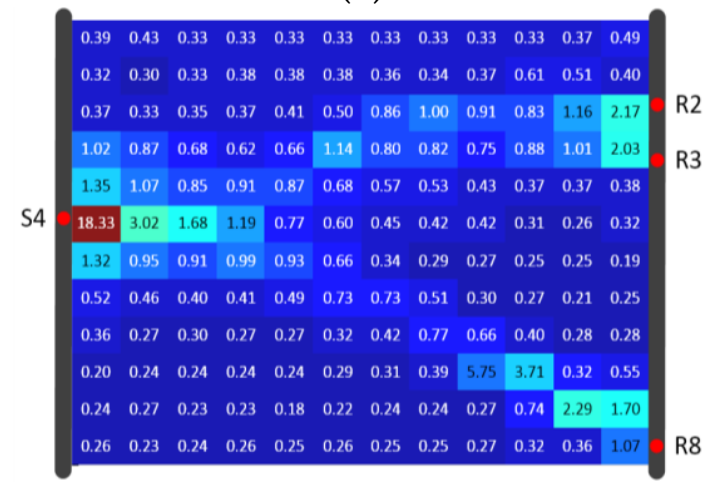

(b)

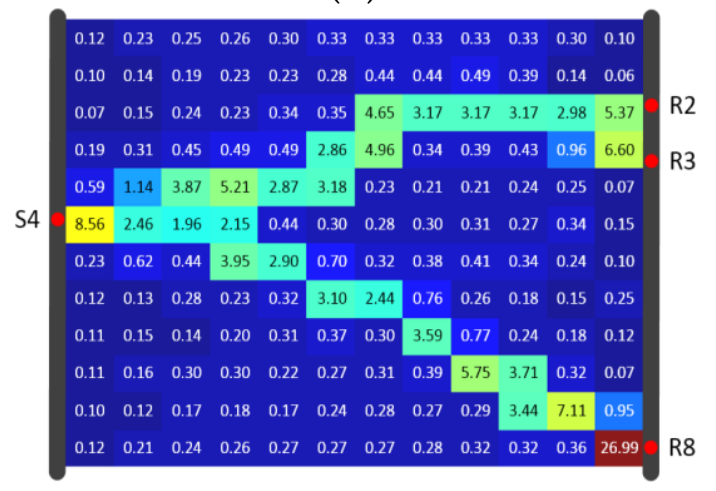

Figure 3.17. Algorithm result comparison for the model under $12 \times 12$ resolution of the (a) SIRT result and (b) SIRT-Cimmino result, shown in diffusivity $\left[\mathrm{m}^{2} \mathrm{~s}^{-1}\right]$. 
(a)

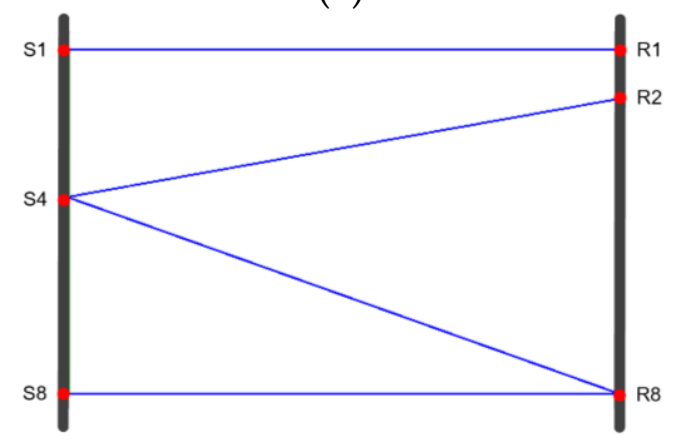

(c)

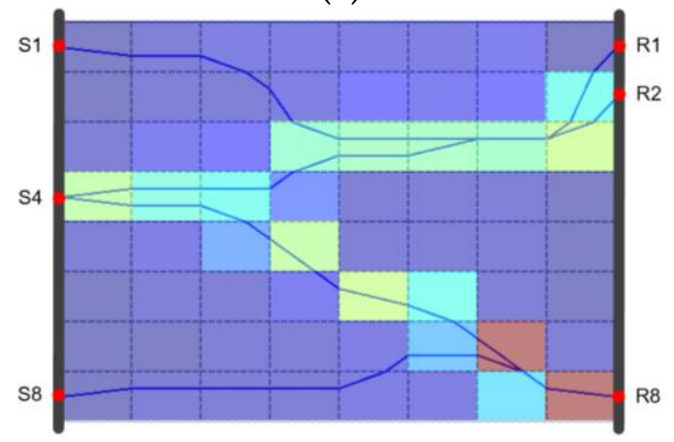

(b)

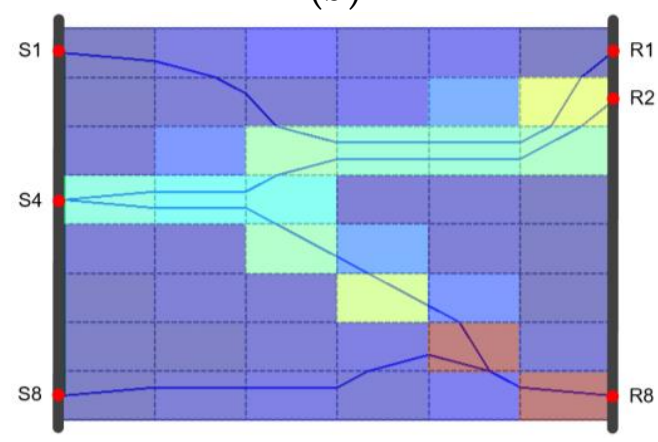

(d)

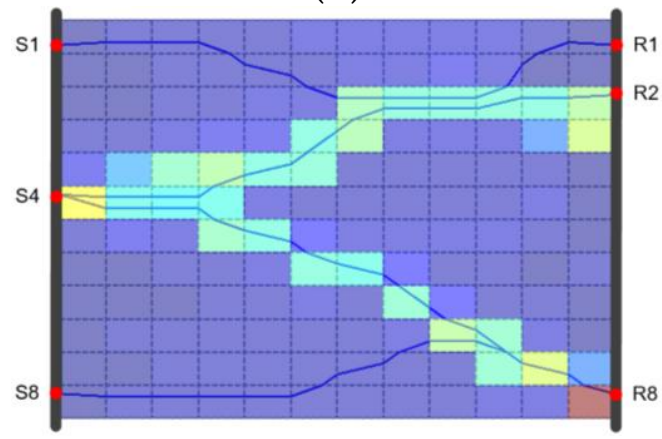

Figure 3.18. The trajectories of ray S1R1, S4R2, S4R8 and S8R8 based on the inversion of $t_{100}$ and SIRT-Cimmino under different resolutions. (a) The ray trajectories in homogeneous aquifer, (b) the ray trajectories in $8 \times 6$ resolution, (c) the ray trajectories in $8 \times 8$ resolution, (d) the ray trajectories in $12 \times 12$ resolution.

Table 3.4. RMSE and correlation coefficient of the model inversion using SIRT and SIRT-Cimmino.

\begin{tabular}{ccccccc}
\hline & \multicolumn{3}{c}{ RMSE } & \multicolumn{3}{c}{ Correlation Coefficient } \\
\cline { 2 - 7 } & $8 \times 6$ & $8 \times 8$ & $12 \times 12$ & $8 \times 6$ & $8 \times 8$ & $12 \times 12$ \\
\hline SIRT & 10.39 & 4.66 & 7.11 & 0.64 & 0.63 & 0.61 \\
\hline SIRT-Cimmino & 7.51 & 8.04 & 10.77 & 0.65 & 0.66 & 0.66 \\
\hline
\end{tabular}

\subsection{Aquifer analogue outcrop study}

The two previous numerical models employed for validating purposes are relatively simple compared to an actual aquifer. We thus use an aquifer analogue outcrop to validate SIRT-Cimmino algorithm. The aquifer analogue outcrop has a size of $16 \mathrm{~m} \times 10 \mathrm{~m} \times 7 \mathrm{~m}$ and is from Herten village in the southwest Germany. The outcrop was first introduced by Bayer (1999). The laboratory measurements 
were carried out by Heinz et al. (2003) and Kostic et al. (2005) to obtain the hydraulic parameters. A geostatistical analysis was conducted by Maji and Sudicky (2008) to yield the 3D hydraulic parameter distribution, which has a fine resolution of $0.05 \mathrm{~m} \times 0.05 \mathrm{~m} \times 0.05 \mathrm{~m}$. For computational reasons, the derived data set was scaled up to $0.1 \mathrm{~m} \times 0.1 \mathrm{~m} \times 0.1 \mathrm{~m}$ and the number of cells reaches ca. 1 million. Figure 3.19 shows a 3D representation of the upscaled distribution of hydraulic diffusivity.

Hu et al. (2011) built a 3D model based on the upscaled data and simulated short-term pumping tests using MODFLOW. They reconstructed the diffusivity distribution of two selected profiles using GeoTom3D software, which is based on SIRT. In this section, TomoGo software is used to implement SIRT-Cimmino algorithm. The performance of SIRT-Cimmino is evaluated and compared with the result of Hu et al. (2011).

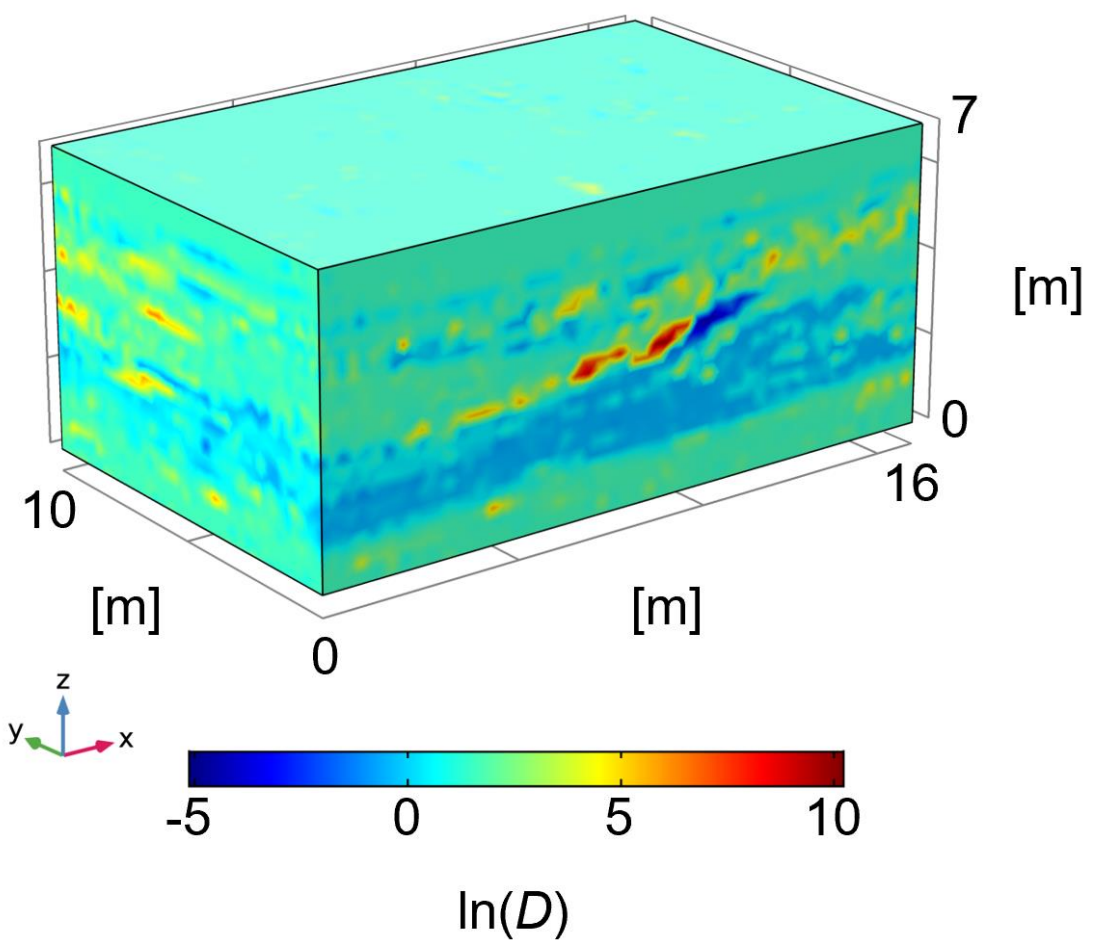

Figure 3.19. The upscaled distribution of the natural logarithm of hydraulic diffusivity $\left[\mathrm{m}^{2} \mathrm{~s}^{-1}\right]$ of the Herten aquifer analogue outcrop with a size of $16 \mathrm{~m} \times 10$ $\mathrm{m} \times 7 \mathrm{~m}$. Visualization effect through software Comsol. 


\subsubsection{Case-study profile description}

We study the same area presented in Hu et al. (2011). As shown in Figure 3.20, four wells (i.e. E, W, S and N) are located at the corners of a square and well C sits in the center with a distance of $2.5 \mathrm{~m}$ from other wells. The diameter and the depth of each well are 0.05 and $7 \mathrm{~m}$, respectively. The study areas are WE profile (between wells $\mathrm{W}$ and $\mathrm{E}$ ) and SN profile (between wells $\mathrm{S}$ and N). Both profiles have a size of $5 \mathrm{~m} \times 7 \mathrm{~m}$ and are characterized by $51 \times 69$ arrays (= 3519 pixels).

As shown in Figure 3.21 and Figure 3.22, both profiles are highly heterogeneous and stratally structured. High diffusivities $\left(>300 \mathrm{~m}^{2} \mathrm{~s}^{-1}\right)$ are mainly distributed in high- $D$ continua (between 3 and $4 \mathrm{~m}$ depth), which are shown in brownish color and highlighted by white lines. The continuum in WE profile is slightly inclined while the continuum in SN profile is horizontal. Low- $D$ zones are found under the high- $D$ continua (i.e., layers we 4 and sn 4 with diffusivity about 1 $\mathrm{m}^{2} \mathrm{~s}^{-1}$ ). The bottom layers (we5 and sn5) and second top layers (we2 and sn2) are mixed with red and green color, and have moderate diffusivity, which is a bit higher than that of the light blue layers at the top of profiles (we1 and sn1). The high- $D$ continuum is considered the primary feature and the four aforementioned layers are secondary features. Apart from this, some inferior features can be neglected during the inversion, for instance, a thin horizontal layer with dark blue color at $6 \mathrm{~m}$ depth of WE profile, and a small dark blue area between 4 and $5 \mathrm{~m}$ depth near well $\mathrm{W}$.

Statistical values of two profiles are listed in Table 3.5 and Table 3.6. The pixels with extremely high diffusivity $\left(>100 \mathrm{~m}^{2} \mathrm{~s}^{-1}\right)$ in high- $D$ continua significantly increase the overall mean and variance, although the profiles are quantitatively dominated by the pixels with diffusivity less than $10 \mathrm{~m}^{2} \mathrm{~s}^{-1}$. 
(a)

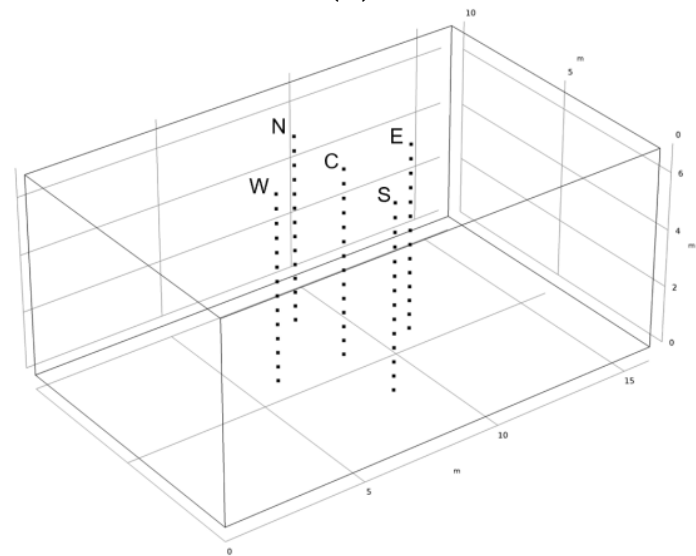

(b)

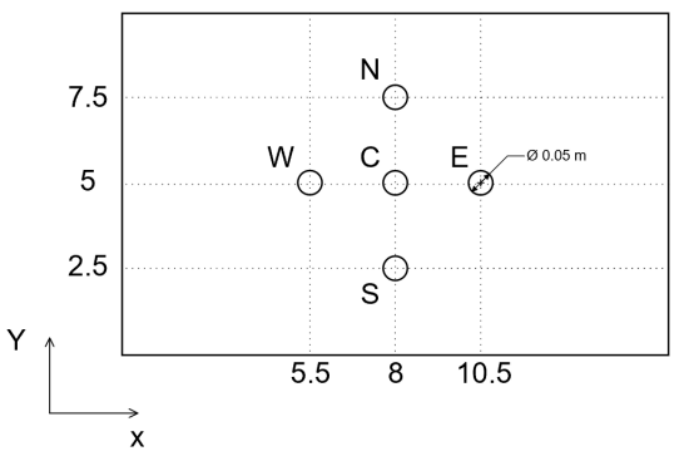

Figure 3.20. (a) Well locations in the studied area. Each well is installed with 14 screens, which are represented by the black squares. (b) The top view of the fivespot well pattern in the studied area.

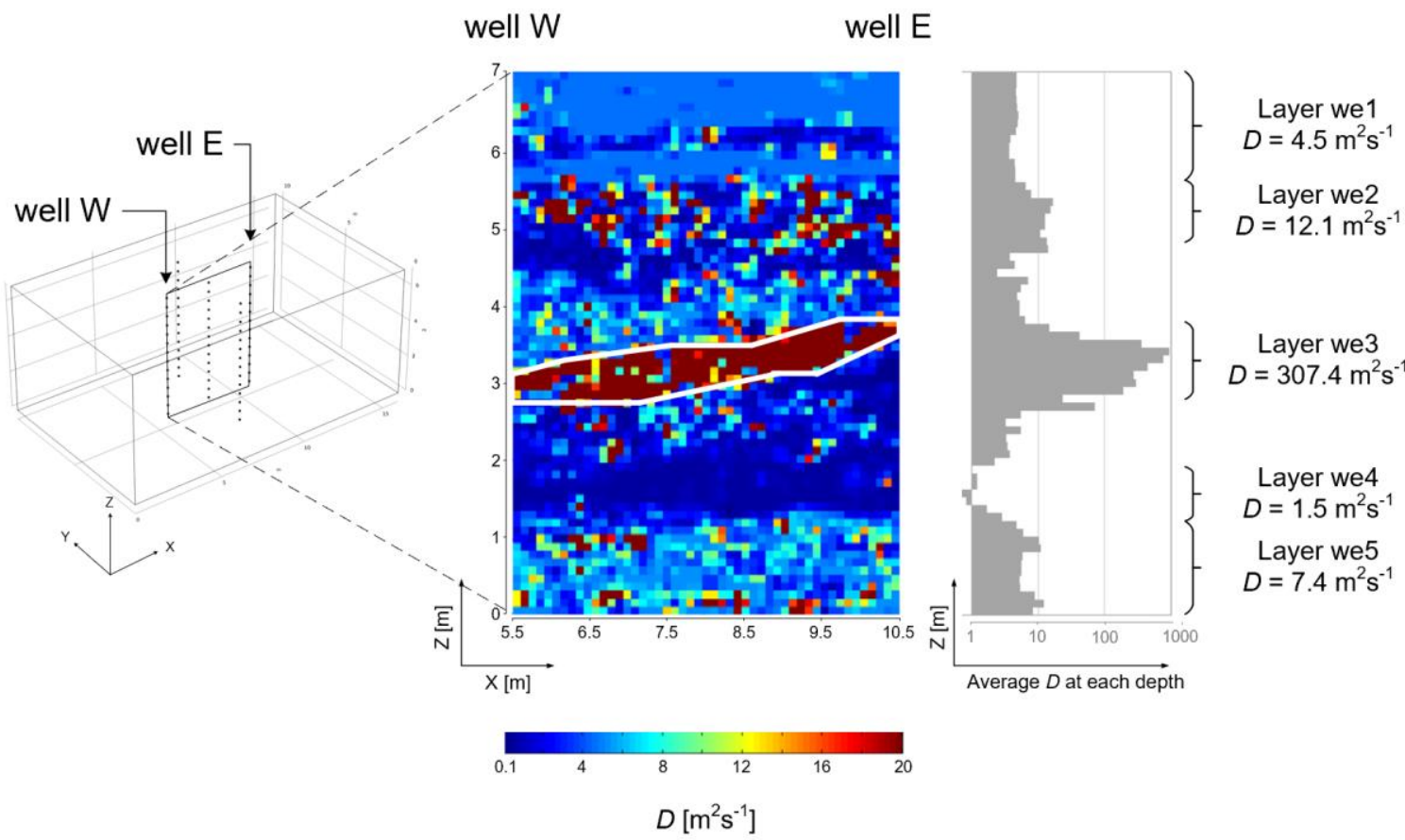

Figure 3.21. The diffusivity distribution of WE profile. The diffusivity values larger than $20 \mathrm{~m}^{2} \mathrm{~s}^{-1}$ are shown in brown. The histogram on the right side shows the average diffusivity at each depth. The inclined high- $D$ continuum highlighted by white lines is the main feature of this profile.

Table 3.5. Statistical values of WE profile.

\begin{tabular}{c|c|c|c}
\hline mean & minimum & maximum & variance \\
\hline $54 \mathrm{~m}^{2} \mathrm{~s}^{-1}$ & $0.1 \mathrm{~m}^{2} \mathrm{~s}^{-1}$ & $1.5 \times 10^{4} \mathrm{~m}^{2} \mathrm{~s}^{-1}$ & $3.2 \times 10^{5} \mathrm{~m}^{4} \mathrm{~s}^{-2}$ \\
\hline
\end{tabular}




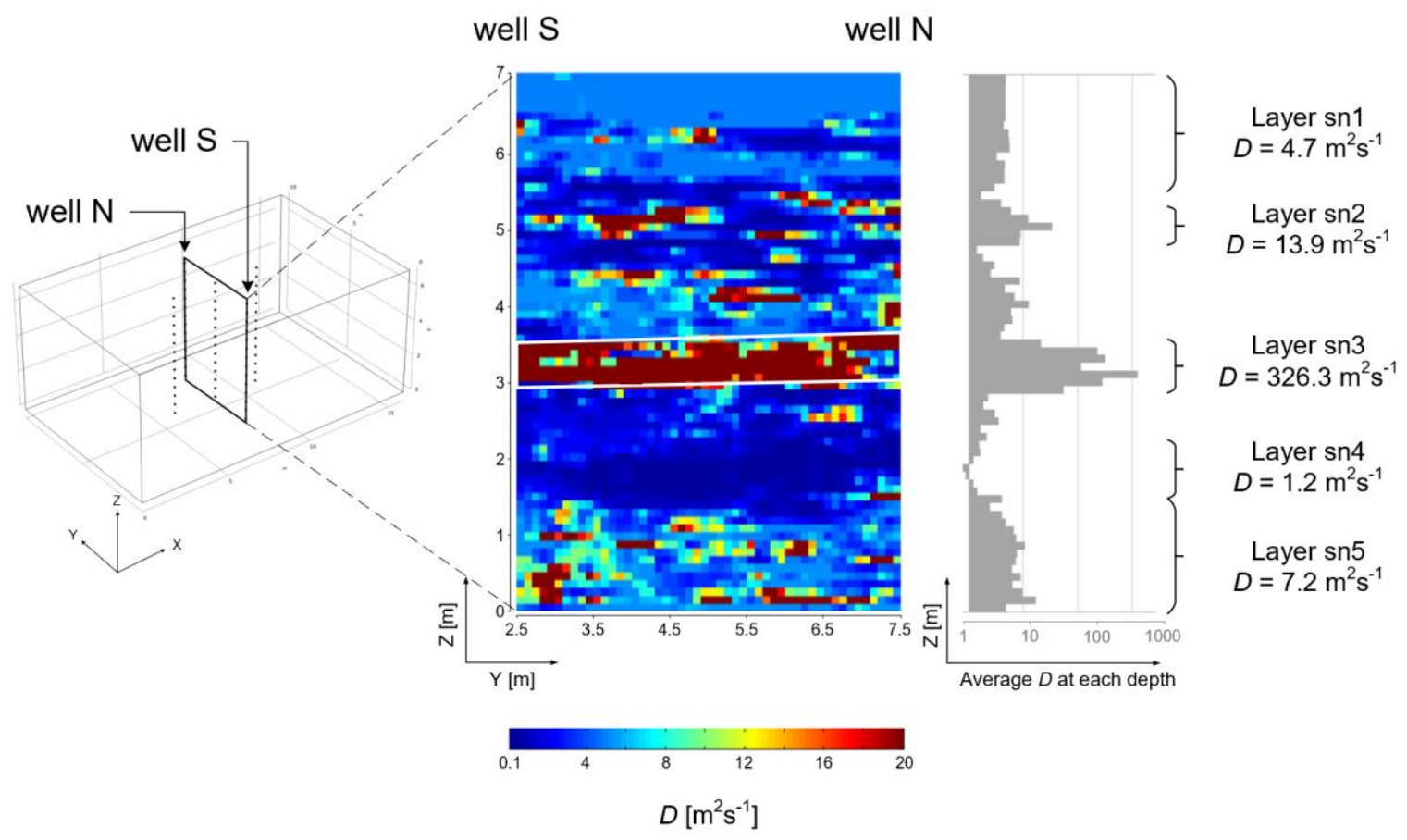

Figure 3.22. The diffusivity distribution of SN profile. The diffusivity values larger than $20 \mathrm{~m}^{2} \mathrm{~s}^{-1}$ are shown in brown. The histogram on the right side shows the average diffusivity at each depth. The horizontal high- $D$ continuum highlighted by white lines is the main feature of this profile.

Table 3.6. Statistical values of SN profile.

\begin{tabular}{c|c|c|c}
\hline mean & minimum & maximum & variance \\
\hline $38 \mathrm{~m}^{2} \mathrm{~s}^{-1}$ & $0.2 \mathrm{~m}^{2} \mathrm{~s}^{-1}$ & $2.1 \times 10^{4} \mathrm{~m}^{2} \mathrm{~s}^{-1}$ & $1.9 \times 10^{5} \mathrm{~m}^{4} \mathrm{~s}^{-2}$ \\
\hline
\end{tabular}

\subsubsection{Previous work}

Hu et al. (2011) installed 14 screen sections in each well (Figure 3.20 (a)) and simulated pumping tests by using software MODFLOW. During each pumping test, water was pumped from a screen section of well C, and the drawdown at all screen sections of four surrounding wells were recorded. $56(14 \times 4)$ drawdown data were obtained from a single test. The pumping interval was then shifted to the adjacent screen section of well $\mathrm{C}$ in subsequent tests. In total, 14 pumping tests were conducted and 784 drawdown data were obtained. The data set was then used to compute travel times and reconstruct the profiles. 
The inversion process was performed by using geophysical software GeoTom3D, which was developed for seismic ray tomography (Jackson and Tweeton 1996). GeoTom3D applies SIRT algorithm to invert seismic wave travel time and compute the seismic wave velocity map by using following integral:

$$
t=\int_{x_{1}}^{x_{2}} \frac{d s}{v(s)}
$$

where $t$ is the seismic wave travel time, $v(s)$ is the velocity at $s, x_{1}$ is the source position, $x_{2}$ is the receiver position (Lo and Inderwiesen 1994). The integral is identical to Eq. 2.24 by replacing $t$ with $\sqrt{c \cdot t_{\text {peak }}}$ and $v(s)$ with $\sqrt{D(s)}$, respectively. The similarity on the formulation validates the application of GeoTom3D on our inversion. A comparison of experimental details between $\mathrm{Hu}$ et al. (2011) and this work is present in Table 3.7.

To determine the proper iteration step, reconstructions at steps 5, 8, 10 and 15 were compared to the true distribution. Under resolution of $14 \times 10$, the visual comparison revealed that the proper inversion step is 8 . Based on this fact, all reconstructions were thus conducted with 8 steps and are shown in Figure 3.23. The evaluation of reconstructions is conducted in sections 3.3.5 and 3.3.6, together with the reconstructions by using SIRT-Cimmino. 
Table 3.7. The comparison of experimental details between Hu et al. (2011) and this work.

\begin{tabular}{c|c|c}
\hline & Hu et al 2011 & this work \\
\hline Pumping well & well C & wells W and N \\
\hline Observation well & wells E, W, S and N & wells E and S \\
\hline Simulation software & MODFLOW & COMSOL \\
\hline Inversion software & GeoTom3D & TomoGo \\
\hline Inversion algorithm & SIRT & $\begin{array}{c}\text { SIRT, SIRT-Cimmino } \\
\text { Ray-tracing } \\
\text { technique }\end{array}$ \\
\hline
\end{tabular}
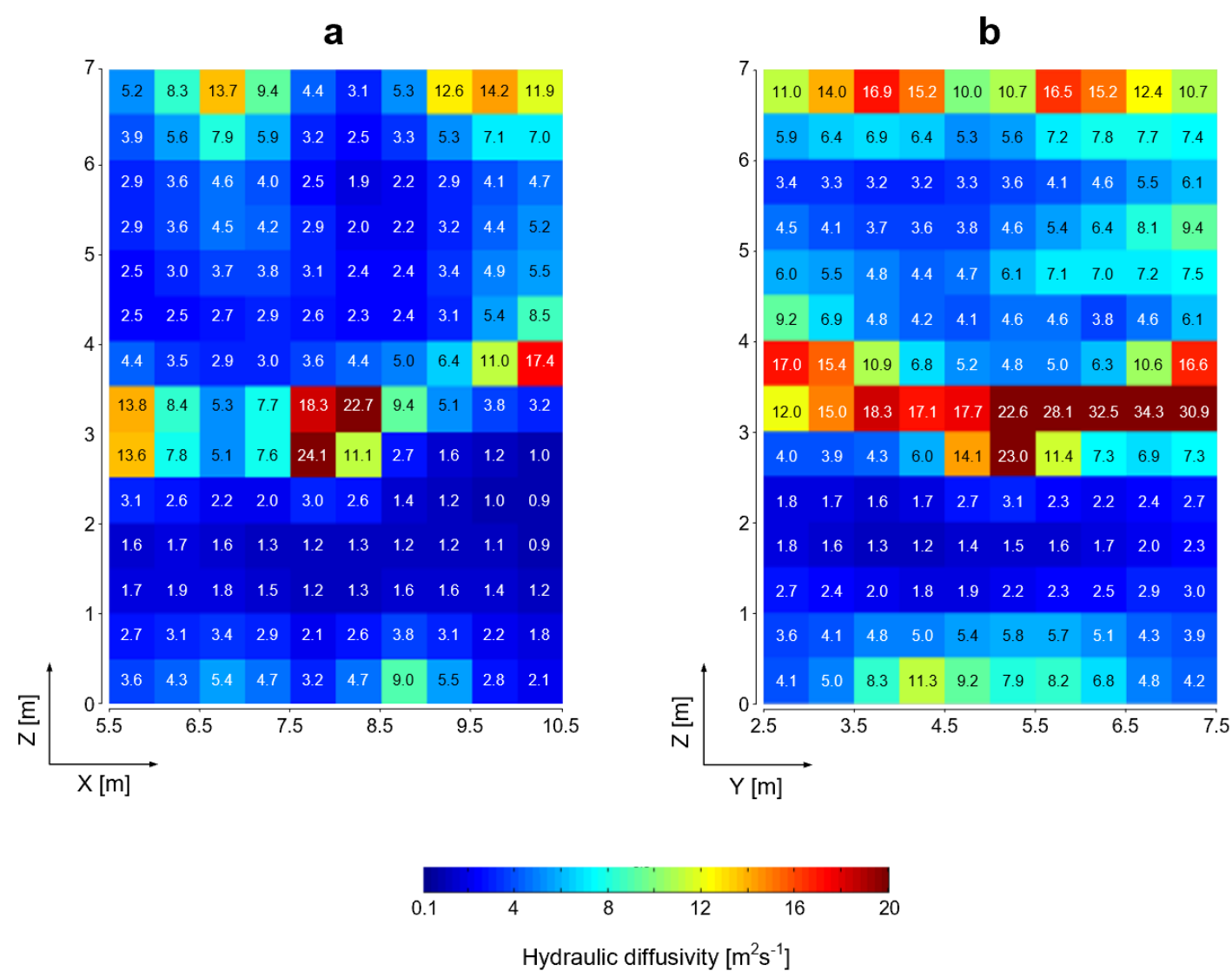

Figure 3.23. The inversion results in Hu et al. (2011). (a) Reconstructed diffusivity of WE profile using $t_{100}$, (b) reconstructed distribution of SN profile using $t_{50}$. 


\subsubsection{Numerical simulation of short term pumping tests}

To compare the result with the previous work, the same areas (profiles WE and $\mathrm{SN}$ ) are studied in this work. For the sake of convenient description, the screens in the wells are numbered from bottom to top, we take well $\mathrm{W}$ as an example, W1 and W14 represent the screen at the bottom and the screen at the top, respectively (Figure 3.24).

The high- $D$ continuum leads to quick pressure response if the pumping and observation sections are located near the high- $D$ continuum $(3 \mathrm{~m}<$ depth $<4 \mathrm{~m})$. The travel time is thus extremely small and close to zero. For instance, the travel times of W9-E8, W9-E7 and W8-E7 are smaller than 0.02 second (Appendix 7.7). As a consequence, numerical differentiation errors are caused. To warrant the accuracy, extra fine mesh and short time step (0.001 second) are used in the simulation. Furthermore, we use wells $\mathrm{W}$ and $\mathrm{N}$ as pumping well, wells $\mathrm{E}$ and $\mathrm{S}$ as observation well, and disuse well C (Figure 3.25). This kind of well setting elongates the distance between pumping and observation wells, and thus avoids extremely small travel times.

Infinite element domain is established in $\mathrm{X}$ - and $\mathrm{Y}$-directions surrounding the outcrop model for the elimination of boundary effect (Figure 3.25), in other words, the depression cone caused by short-term pumping tests can not reach the outer boundaries. The initial head and the constant head at the boundaries are set $0.2 \mathrm{~m}$ above the aquifer top to meet the confined condition. Each test lasts 5 seconds, and the drawdown is recorded every 0.001 second.

The travel times are computed using the numerical differentiation function in MATLAB (Figure 3.26) and listed in Appendixes 7.7 and 7.8. They vary from 0.006 to 2.302 second for WE profile, and from 0.002 to 1.661 second for SN profile. 

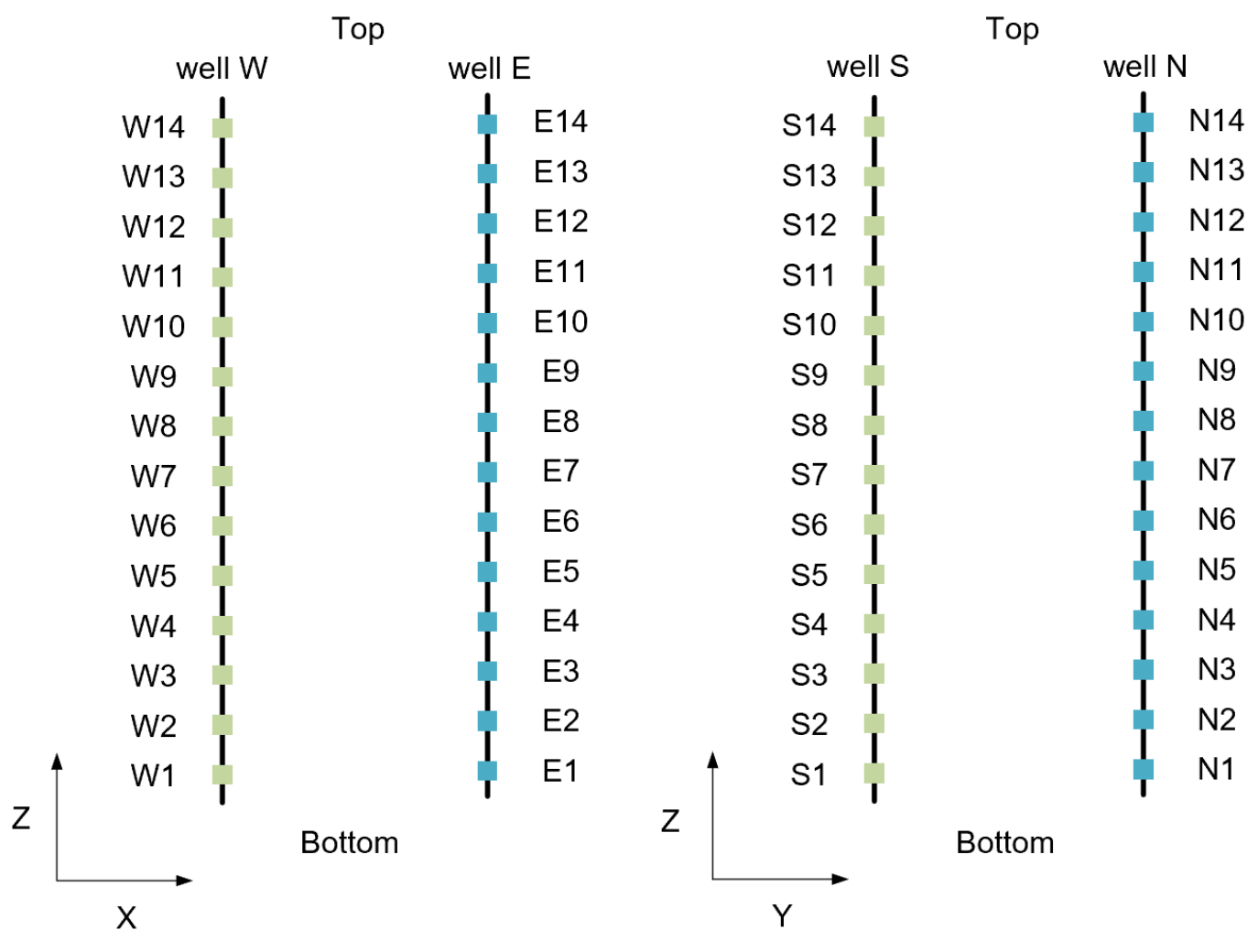

Pumping location

Oberservation location

Figure 3.24. The screens are numbered from bottom to top. The green squares represent the pumping screen sections while the blue squares represent the observation screen sections. Well C is not used and thus not shown in this figure. 


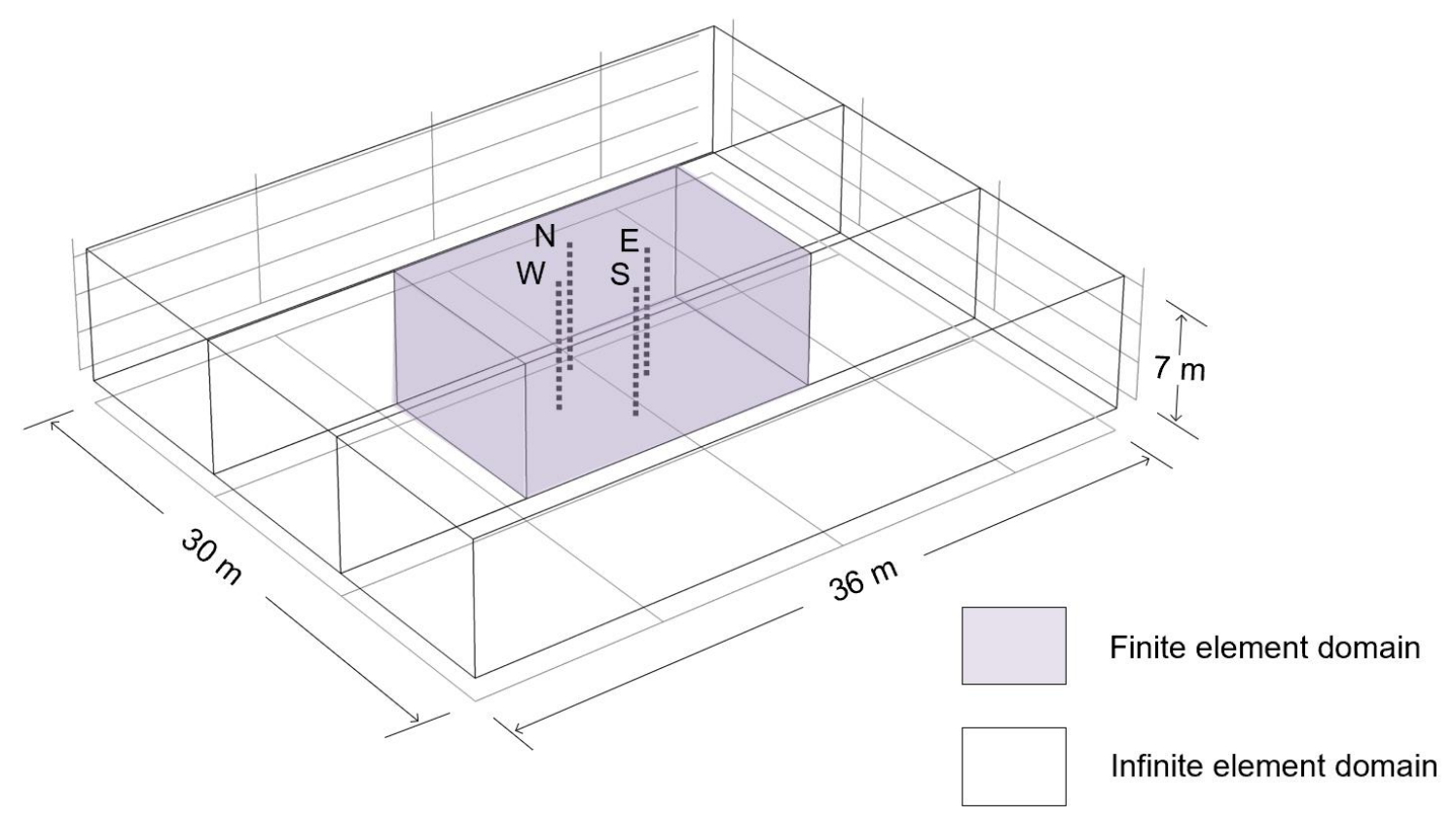

Figure 3.25. The arrangement of four wells (i.e. W, E, N and S). The model is characterized by finite elements (in light purple color) and surrounded by the infinite element domain (in transparence). The outer size of the domain is $36 \mathrm{~m} \times$ $30 \mathrm{~m} \times 7 \mathrm{~m}$.

a

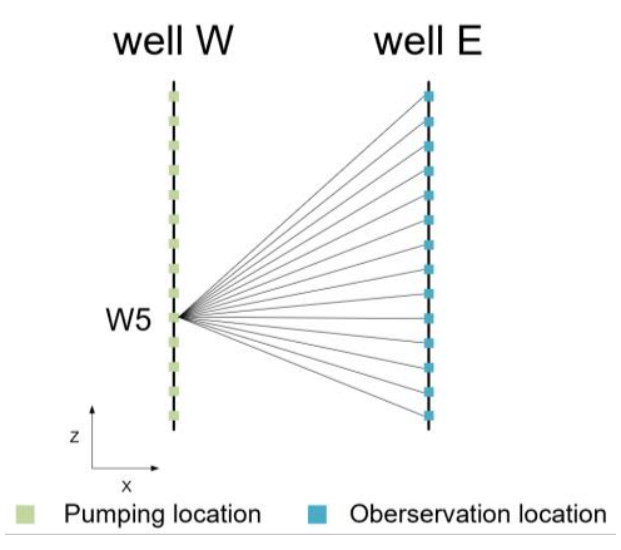

b

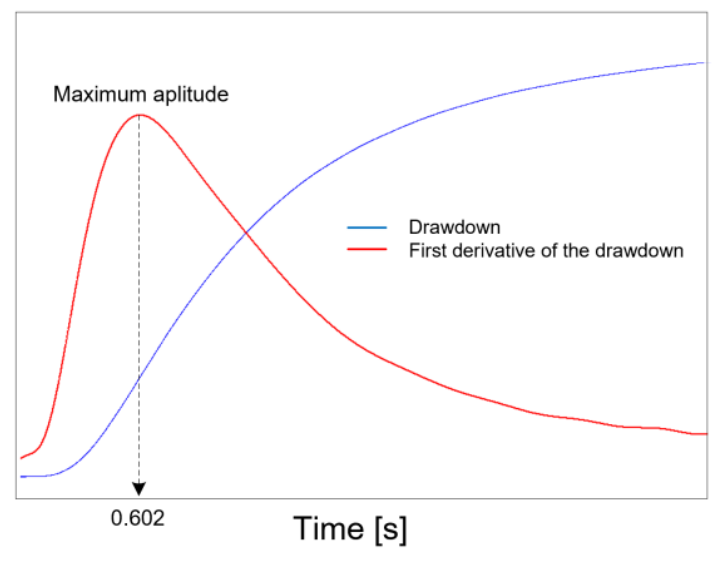

Figure 3.26. (a) A scheme of the cross-well multi-level pumping test at W5 (pumping screen section) and 14 observations in well E. (b) The computation of the travel time (0.602 s) from the drawdown observed at E9. 


\subsubsection{Dilemma of SIRT}

This section examines the dependence of reconstructions on the number of iteration steps (NIS). We take the inversion for WE profile as an example. The mean $\left(54 \mathrm{~m}^{2} \mathrm{~s}^{-1}\right)$, minimum $\left(0.1 \mathrm{~m}^{2} \mathrm{~s}^{-1}\right)$ and maximum $\left(1.5 \times 10^{4} \mathrm{~m}^{2} \mathrm{~s}^{-1}\right)$ are assigned to the initial value, min-constraint and max-constraint in the inversion, respectively. This setting strategy restricts the inversion process and voids the occurrence of uncertainty (e.g., extremely large $D$ ). Note that this setting only suits theoretical analysis, since it is probably impossible to obtain min and max values in practice.

Straight ray method is used in the 1st iteration, and network method is used in the subsequent 50 iterations. Figure 3.27 illustrates residuals with respect to the 50 iteration steps. The residual decreases rapidly and converges to a nonzero. However, RMSE between the true distribution and reconstructed distribution increases in a staged manner as the iteration proceeds (Figure 3.28). A reasonable explanation for the staged manner is the max-constraint. As iteration processes, the value of cell A (the position of cell A is shown in Figure 3.29 (f)) increases until it reaches the maximum at step 18. Due to the max-constraint, the value equals the maximum in the subsequent iterations. At steps 30 and 49, the same problem occurs in cells B and C, respectively (the positions of cells B and C are shown in Figure 3.29 (f)). In other words, a cell has maximum value between steps 18 and 29, two cells have maximum value between steps 30 and 48, and after step 49, three cells have maximum value. These three step-intervals correspond the $2 \mathrm{nd}$, $3 \mathrm{rd}$ and 4th stages in Figure 3.28. It indicates that extremely high values can rise RMSE

significantly, meanwhile the max-constraint restricts the RMSE and leads to a staged increase. On the account of RMSE, the inversion should be conducted within 10 steps. 


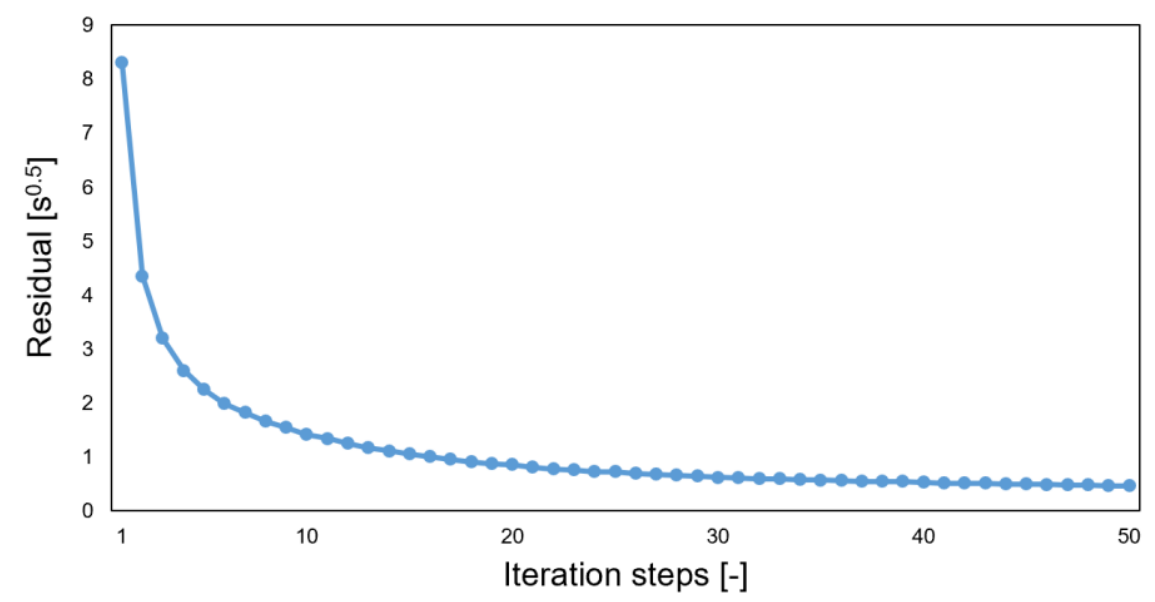

Figure 3.27. Residuals for 50 iteration steps by using SIRT under $14 \times 14$ resolution for WE profile reconstruction.

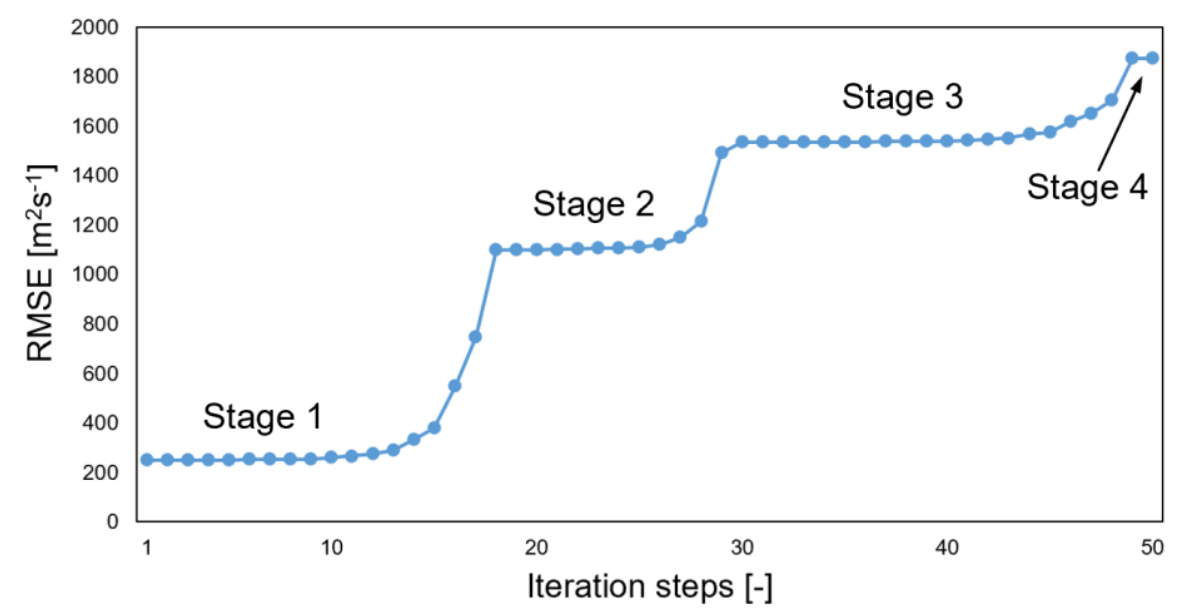

Figure 3.28. RMSE of the true distribution and SIRT based reconstruction at each step. The RMSE shows a staged manner, where first steps of $2 \mathrm{nd}, 3 \mathrm{rd}$ and $4^{\text {th }}$ stages are 18, 30 and 49, respectively.

Figure 3.29 presents the reconstructed diffusivity tomograms at steps 5, 8 10, 15 , and 50. The reconstructions at steps 5, 8 and 10 can not exhibit the continuity of the high- $D$ continuum because the values in the center are much lower than that near the wells. The reconstruction at step 15 shows an opaque image of the continuity where the central cells rise to ca. $15 \mathrm{~m}^{2} \mathrm{~s}^{-1}$. The reconstruction at step 50 exhibits a clear high- $D$ continuum. Based on this fact, the inversion should be iterated more than 15 steps. 
Less steps $(<10)$ yields a small RMSE, meanwhile more steps $(>15)$ gives better primary feature. This dilemma is the main drawback of SIRT algorithm. Due to the complexity of nonlinear system, the existence and uniqueness of the solution under a general condition can not be proven theoretically. It is very likely that the nonlinear system converges to a wrong solution, which has a low residual, high values near the wells (a high RMSE), and low values in the center (an opaque high$D$ continuum). 
(a) True distribution

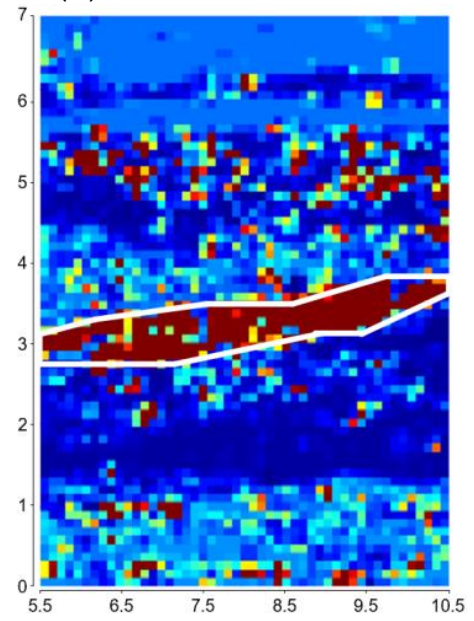

(d) 10 steps

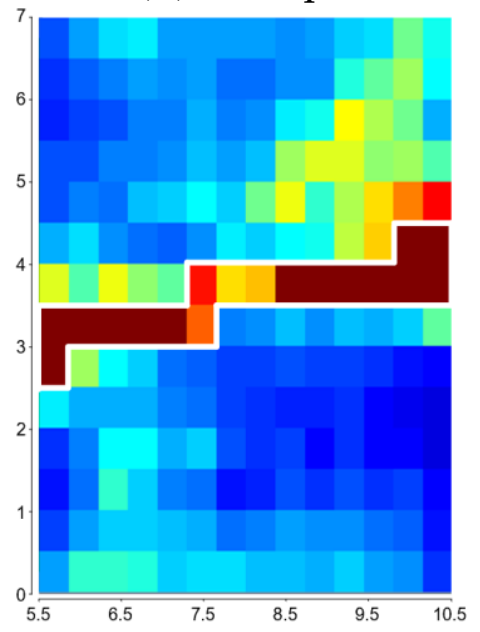

(b) 5 steps

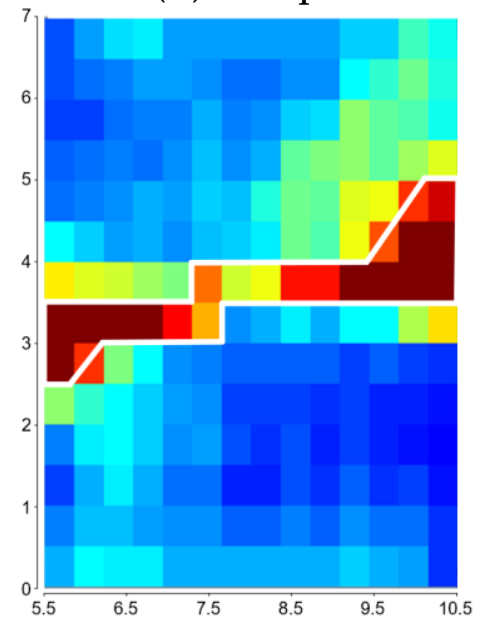

(e) 15 steps

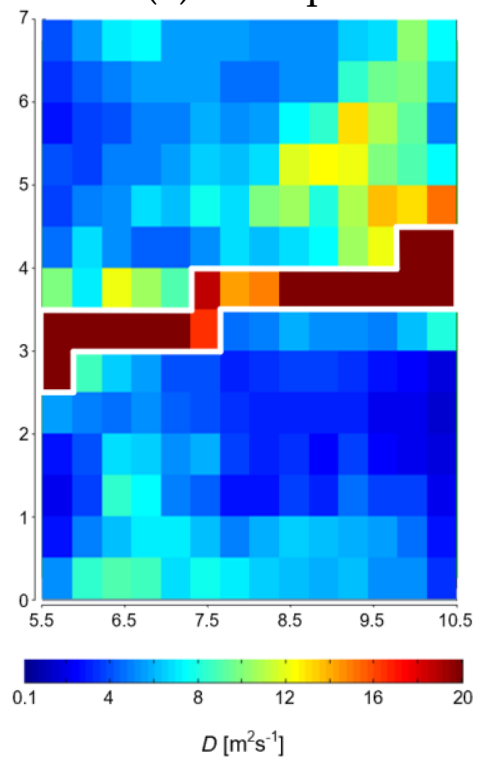

(c) 8 steps

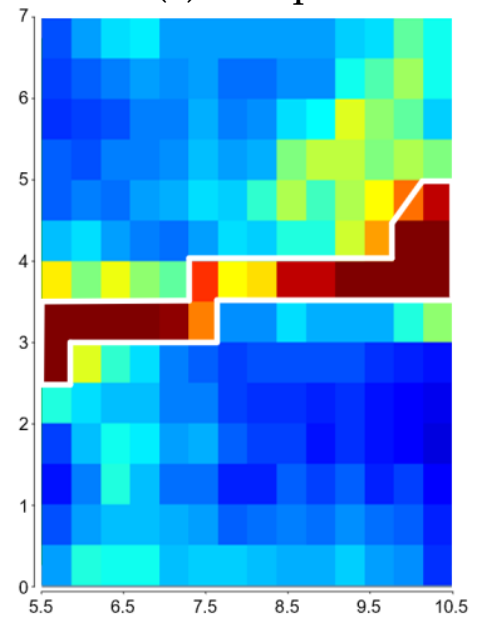

(f) 50 steps

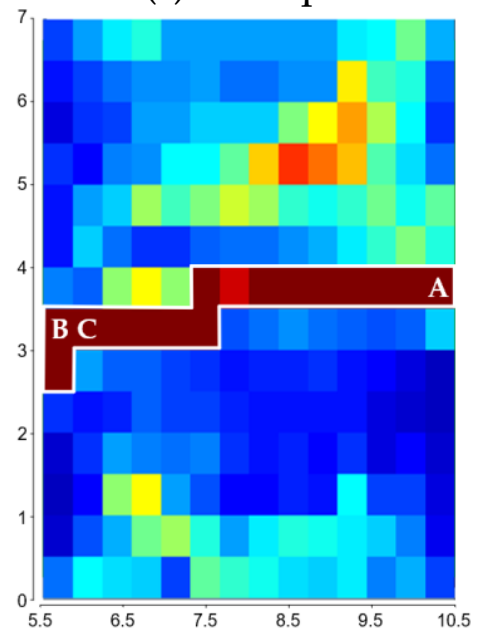

Figure 3.29. Diffusivity $\left[\mathrm{m}^{2} \mathrm{~s}^{-1}\right]$ tomograms of WE profile based on SIRT with different NIS under resolution $14 \times 14$. (a) The true distribution, $(\mathbf{b})-(\mathbf{f})$ the reconstructions at steps $5,8,10,15$ and 50 , respectively. In (f), the values of the cells $\mathrm{A}, \mathrm{B}$ and $\mathrm{C}$ reach the max-constraint, i.e. $1.5 \times 10^{4} \mathrm{~m}^{2} \mathrm{~s}^{-1}$.

\subsubsection{Reconstruction for WE profile}

This section exhibits the results for WE profile by using SIRT-Cimmino. The initial value and constraints are set according to the statistic, i.e. initial value 54 $\mathrm{m}^{2} \mathrm{~s}^{-1}$, min-constraint $0.1 \mathrm{~m}^{2} \mathrm{~s}^{-1}$, max-constraint $1.5 \times 10^{4} \mathrm{~m}^{2} \mathrm{~s}^{-1}$. The result selection 
rule is applied to determine the proper NIS from 51 iteration steps (straight ray method $\times 1$, network method $\times 50$ ).

We first compare the reconstructions under resolution $14 \times 10$ with the previous study and then exhibit the reconstructions under high resolutions. Figure 3.30 shows the true distribution, result of previous work (by using SIRT) and the result of this work (by using SIRT-Cimmino). Table 3.8 shows the average diffusivities in five abovementioned layers. The combination of Figure 3.30 and Table 3.8 provides two comparison ways: structural similarity and the numerical accuracy.

(a) true distribution

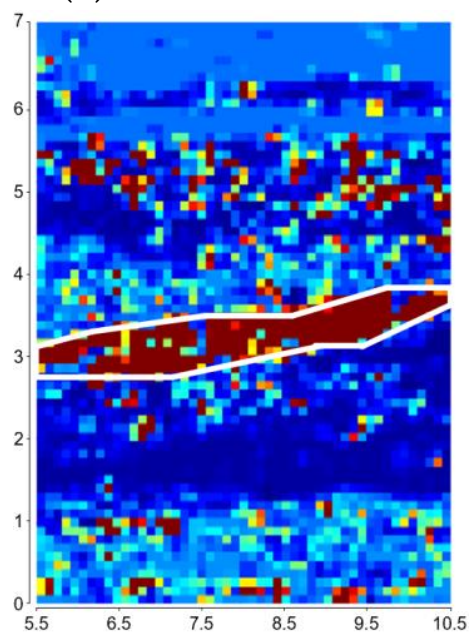

(b) SIRT (Hu et al. 2011)
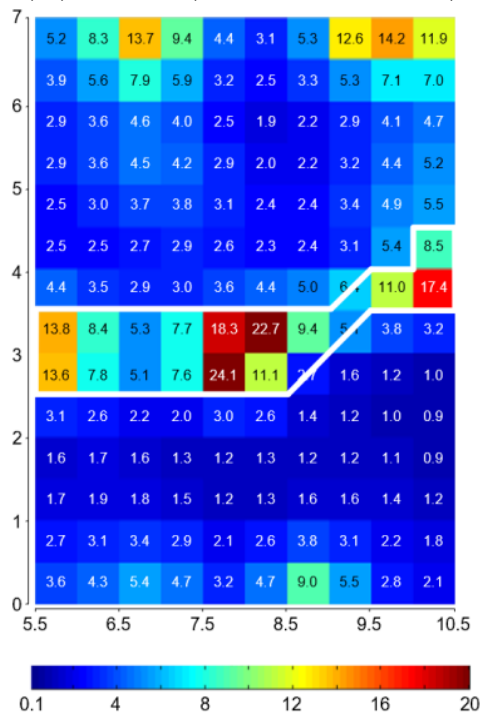

$D\left[\mathrm{~m}^{2} \mathrm{~s}^{-1}\right]$ (c) SIRT-Cimmino

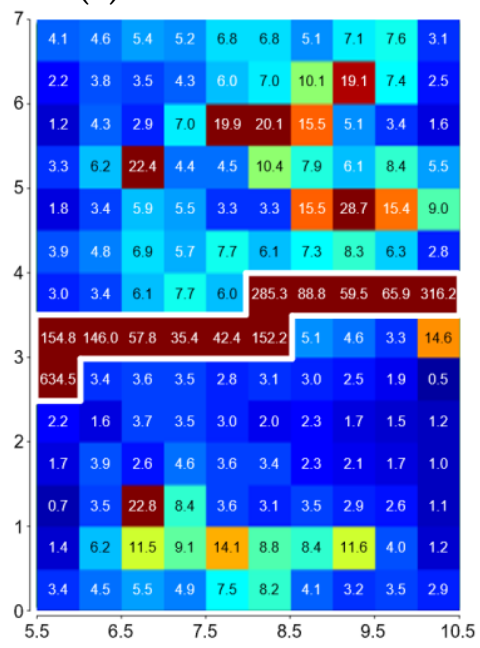

Figure 3.30. The comparison of (a) true distribution of profile WE, (b) the reconstructed distribution by using SIRT (Hu et al. 2011), and (c) the reconstructed distribution by using SIRT-Cimmino. 
Table 3.8. The comparison of average diffusivities $\left[\mathrm{m}^{2} \mathrm{~s}^{-1}\right]$ of different layers in true distribution of WE profile, the reconstructed distribution by using SIRT (Hu et al. 2011), and the reconstructed distribution by using SIRT-Cimmino.

\begin{tabular}{c|c|c|c}
\hline Layer & $\begin{array}{c}\text { True } \\
\text { distribution }\end{array}$ & $\begin{array}{c}\text { SIRT } \\
\text { (Hu et al. 2011) }\end{array}$ & $\begin{array}{c}\text { SIRT-Cimmino } \\
\text { (this work) }\end{array}$ \\
\hline we1 & 4.5 & 5.8 & 6.8 \\
\hline we2 & 12.1 & 3.5 & 8.5 \\
\hline we3 & 307.4 & 7.8 & 70.6 \\
\hline we4 & 1.5 & 1.4 & 3.5 \\
\hline we5 & 7.4 & 3.2 & 6.0 \\
\hline
\end{tabular}

The evaluation can be summarized as follows:

- Primary feature. The inclined high-D continua in both tomograms are correctly located in spite of the coarse resolution. However, the continuity in SIRT result is damaged by low values of some cells within the continuum. The average diffusivity in the continuum (we3) is only 7.8 $\mathrm{m}^{2} \mathrm{~s}^{-1}$, while the true value is $307.4 \mathrm{~m}^{2} \mathrm{~s}^{-1}$. This numerical inaccuracy influences the contrast between the high- $D$ continuum and other layers.

- Secondary features. The low-D layers (we4) under the continua in both results are well rebuilt. The average diffusivity of SIRT result $\left(1.4 \mathrm{~m}^{2} \mathrm{~s}^{-1}\right)$ is slightly closer to the true value $\left(1.5 \mathrm{~m}^{2} \mathrm{~s}^{-1}\right)$, compared to that of SIRTCimmino result $\left(3.5 \mathrm{~m}^{2} \mathrm{~s}^{-1}\right)$. The thickness of the bottom layer (we5) in SIRT result is reduced to half of its true thickness. The layer we2 using SIRT does not show the heterogeneity within the layer. Moreover, this layer should have the second highest diffusivity, which is higher than that of the top layer (we1). These two numerical characteristics are present in the SIRT-Cimmino result but absent in the SIRT result. 
- Inferior features. The dark blue thin horizontal layer at $6 \mathrm{~m}$ depth and the blue small area at $5 \mathrm{~m}$ depth near well $\mathrm{W}$ can not be rebuilt using either of two algorithms.

The evaluation shows SIRT-Cimmino provides better structural similarity and higher numerical accuracy than SIRT.

The resolution plays a vital role for the tomogram quality. High resolution helps to detect the small scaled variability (e.g. inferior features, layer thickness, and layer dip) at the expense of the reconstruction reliability. Various high resolutions are tested, Figure 3.31 shows the reconstructions with the resolutions ranging from $14 \times 10$ to $21 \times 20$. 
(a) true distribution

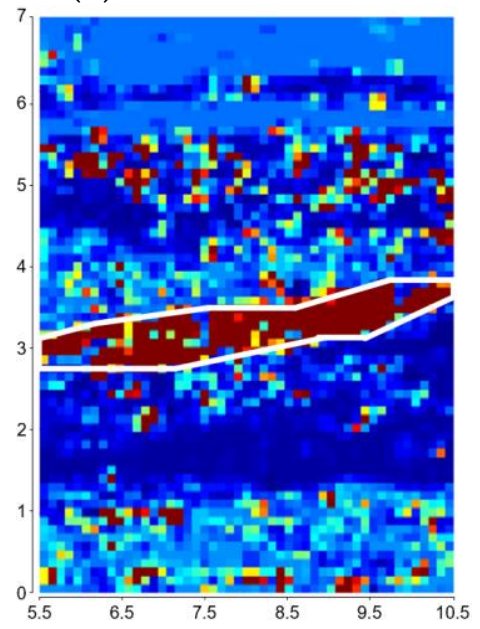

(d) $21 \times 10$

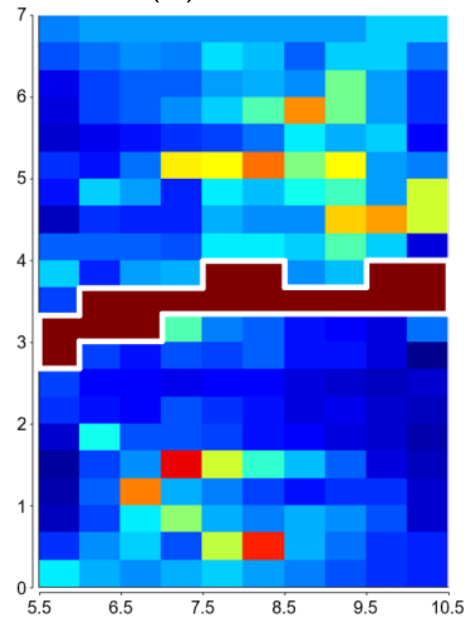

(b) $14 \times 10$

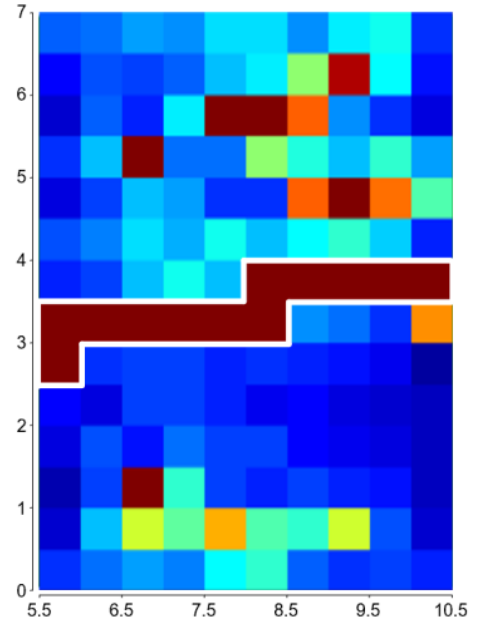

(e) $21 \times 15$

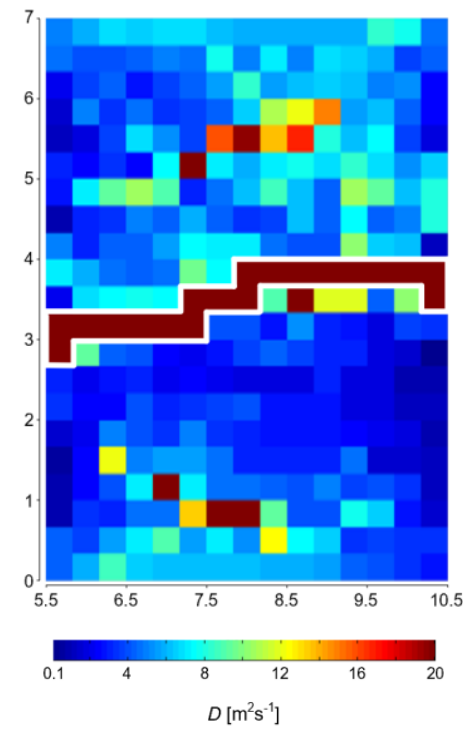

(c) $14 \times 14$

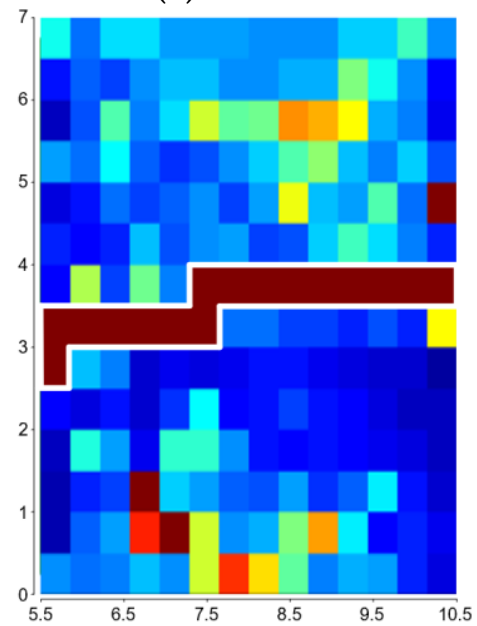

(f) $21 \times 20$

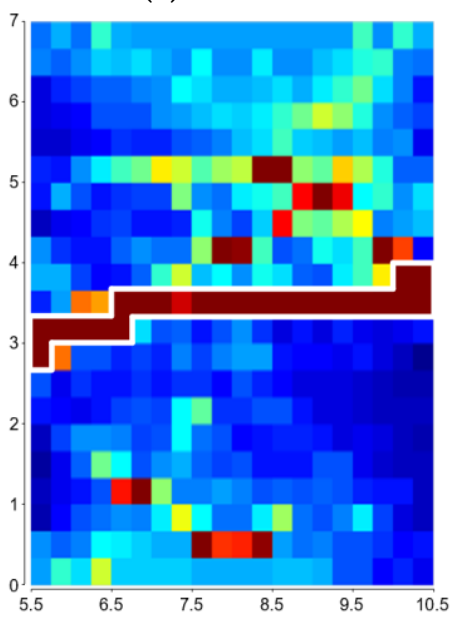

Figure 3.31. Comparison of (a) the true distribution of WE profile, and the reconstructed distributions under resolutions of (b) $14 \times 10$, (c) $14 \times 14$, (d) $21 \times$ 10, (e) $21 \times 15$ and (f) $21 \times 20$.

The high- $D$ continuum is presented in each tomogram, but the shape is slightly deviated in the vertical direction under resolutions $21 \times 10$ and $21 \times 15$. Secondary features are all clear rebuilt in each tomogram. As an inferior feature, the blue small area at $5 \mathrm{~m}$ depth near well $\mathrm{W}$ is detected under high resolutions, while the dark blue thin horizontal layer at the top, is not recognized under any resolutions. 
Software TomoGo visualizes the trajectory of the signal. As shown in Figure 3.32, the trajectories are fine described under higher resolutions.

(a) $14 \times 10$

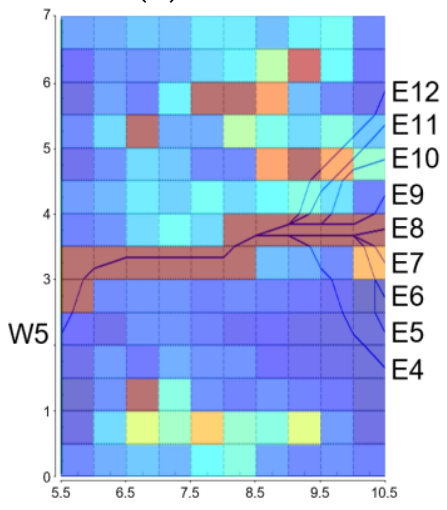

(b) $21 \times 10$

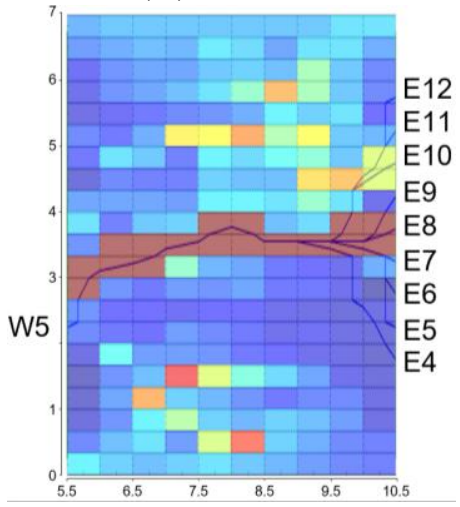

(c) $21 \times 20$

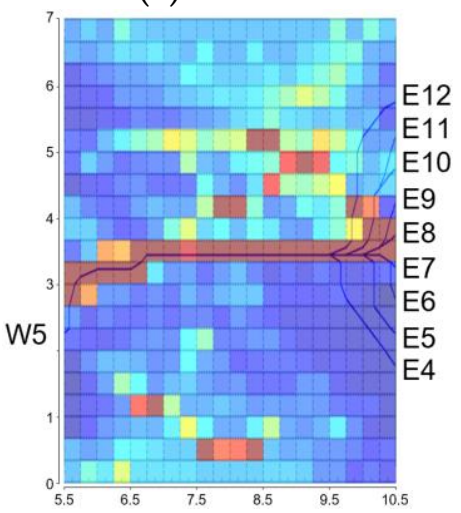

Figure 3.32. The trajectories from W5 to E4 - E12 in WE profile by using SIRTCimmino under three different resolutions, (a) $14 \times 10$, (b) $21 \times 10$ and (c) $21 \times 20$.

\subsubsection{Reconstruction for $\mathrm{SN}$ profile}

This section exhibits the results for SN profile by using SIRT-Cimmino. According to the statistics, the initial value, min-constraint and max-constraint are set as $38 \mathrm{~m}^{2} \mathrm{~s}^{-1}, 0.20 \mathrm{~m}^{2} \mathrm{~s}^{-1}$ and $2.10 \times 10^{4} \mathrm{~m}^{2} \mathrm{~s}^{-1}$ respectively. The result selection rule is applied to determine the proper NIS from 51 iteration steps (straight ray method $\times 1$, network method $\times 50$ ).

We first compare the reconstructions under resolution $14 \times 10$ with the previous study and then exhibit the reconstructions under high resolutions. Figure 3.30 shows the true distribution, result of Hu et al. (2011) (by using SIRT) and the result of this work (by using SIRT-Cimmino). Table 3.9 shows the average diffusivities in five layers. The combination of Figure 3.30 and Table 3.9 provides comparison regarding to the structural similarity and the numerical accuracy. 
(a) true distribution

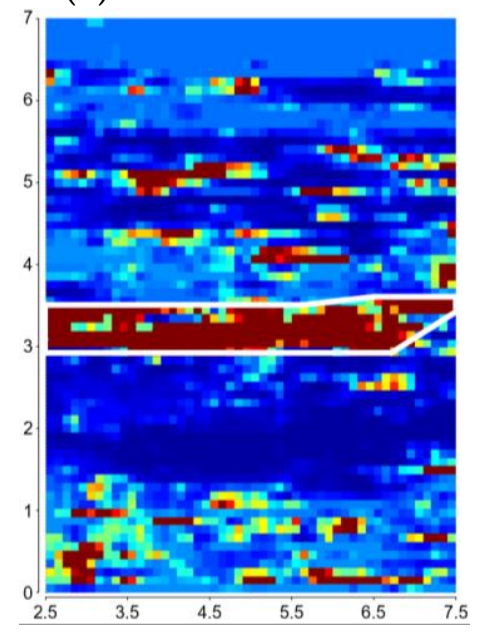

(b) SIRT (Hu et al. 2011)

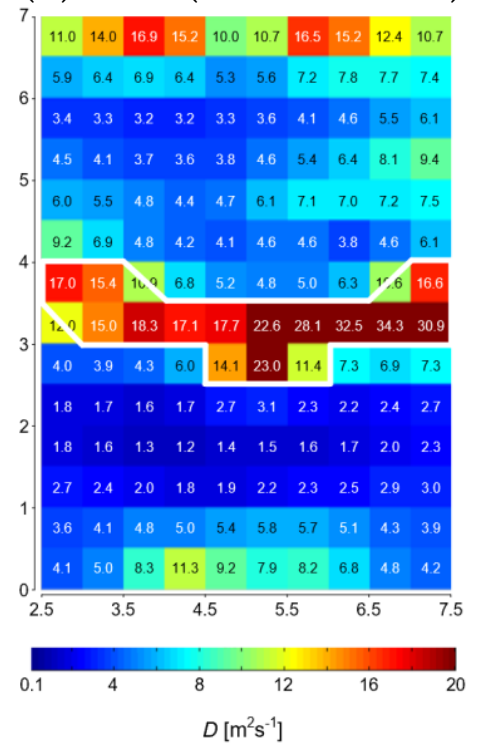

(c) SIRT-Cimmino

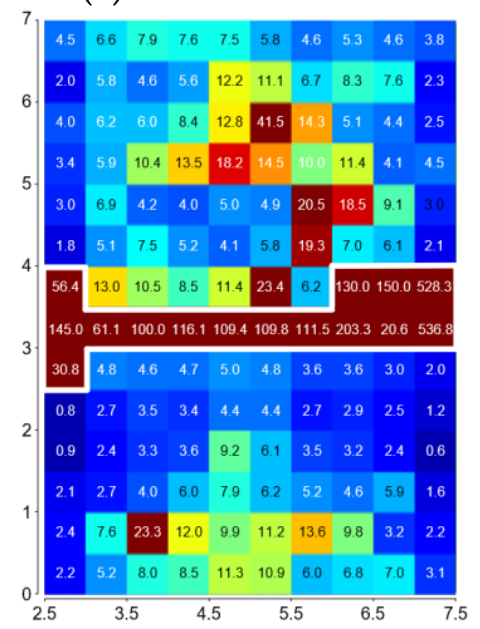

Figure 3.33. The comparison of (a) true distribution of $\mathrm{SN}$ profile, (b) the reconstructed distribution by using SIRT (Hu et al. 2011), and (c) the reconstructed distribution by using SIRT-Cimmino.

Table 3.9. The comparison of average diffusivities $\left[\mathrm{m}^{2} \mathrm{~s}^{-1}\right]$ of different layers in true distribution of SN profile, the reconstructed distribution by using SIRT ( $\mathrm{Hu}$ et al. 2011), and the reconstructed distribution by using SIRT-Cimmino.

\begin{tabular}{c|c|c|c}
\hline Layer & $\begin{array}{c}\text { True } \\
\text { distribution }\end{array}$ & $\begin{array}{c}\text { SIRT } \\
\text { (Hu et al. 2011) }\end{array}$ & $\begin{array}{c}\text { SIRT-Cimmino } \\
\text { (this work) }\end{array}$ \\
\hline sn1 & 4.7 & 7.9 & 6.2 \\
\hline sn2 & 13.9 & 5.7 & 9.5 \\
\hline sn3 & 326.3 & 16.4 & 129.3 \\
\hline sn4 & 1.2 & 1.9 & 3.7 \\
\hline sn5 & 7.2 & 4.7 & 6.9 \\
\hline
\end{tabular}

The evaluation can be summarized as follows:

- Primary feature. The horizontal high-D continua in both tomograms are correctly located in the middle depth, but none of them show the precise shape, especially, the shape of the joints to two wells. From the perspective of numerical values, the average diffusivity in the continuum 
(we3) of SIRT result is only $16.4 \mathrm{~m}^{2} \mathrm{~s}^{-1}$, while the true value is $326.3 \mathrm{~m}^{2} \mathrm{~s}^{-1}$. Due to low values within the reconstructed continuum, the top layer might be mistaken for another high- $D$ continuum.

- Secondary features. SIRT result rebuilds the low-D layer (we4) with a better shape. The layer we2 using SIRT does not show the heterogeneity within the layer. Moreover, this layer should have the second highest diffusivity, which is higher than that of the top layer (we1). These two numerical characteristics are present in the SIRT-Cimmino result but absent in the SIRT result. None of the tomograms rebuild the upper part of the top layer (we1), i.e., the first row of the cells, correctly.

The evaluation shows SIRT-Cimmino provides better structural similarity and higher numerical accuracy than SIRT.

Figure 3.34 shows the reconstructions with the resolutions ranging from $14 \times$ 10 to $21 \times 20$. Each resolution presents the high- $D$ continuum with correct direction. The shape of joints to the wells can not precisely estimated. Under the resolution $21 \times 20$, the joint to well $\mathrm{N}$ even presents a shape of "fist with a hole", which consist of three cells with yellowish color. The cause can be explained through the visualization of the trajectories (Figure 3.35 (c)). Due to the strong nonuniqueness under high resolution, the signals do not traverse through these three cells, where the diffusivities are low. As an secondary feature, the low- $D$ zone beneath the high- $D$ continuum is too vague under each resolution. Fortunately, thanks to the high value contrast, we still can distinguish this low- $D$ layer. 
(a) true distribution

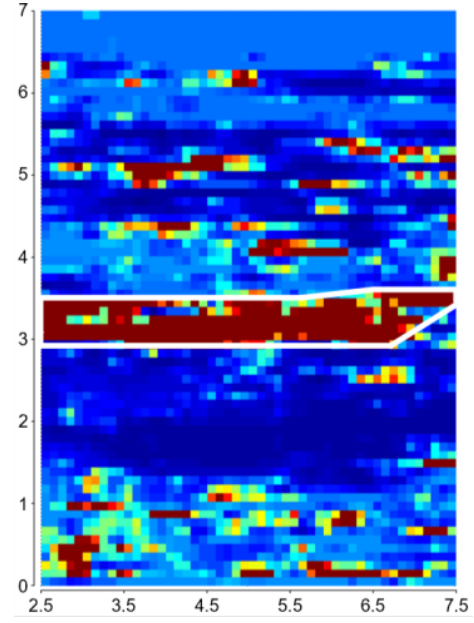

(d) $21 \times 10$

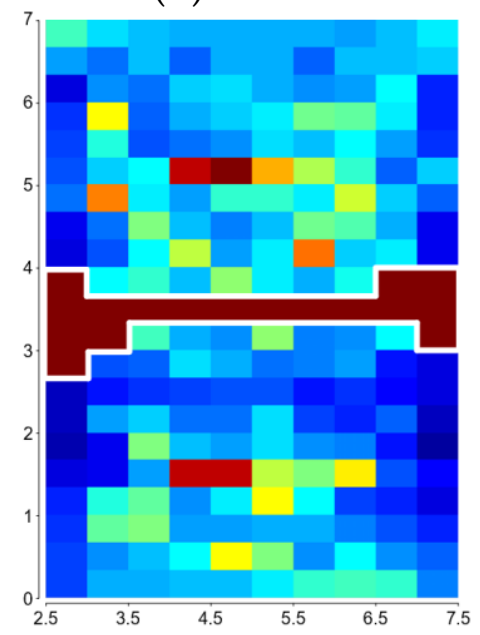

(b) $14 \times 10$

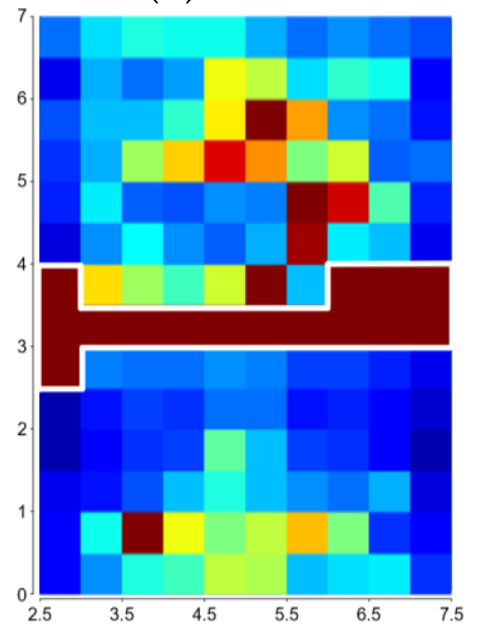

(e) $21 \times 15$

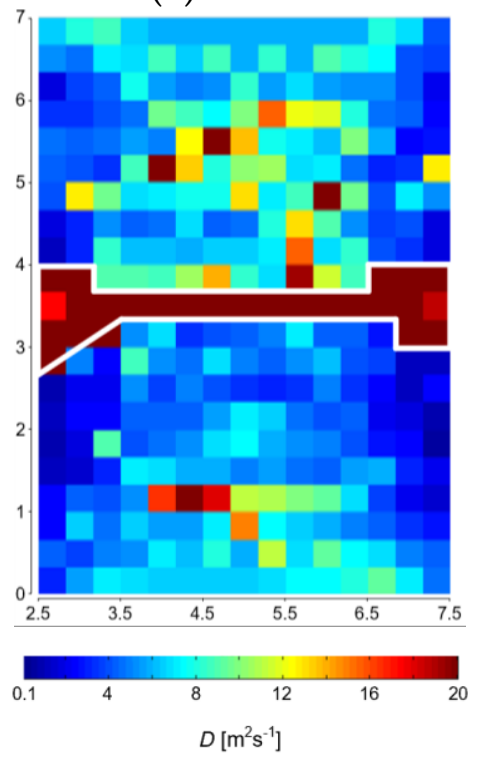

(c) $14 \times 14$

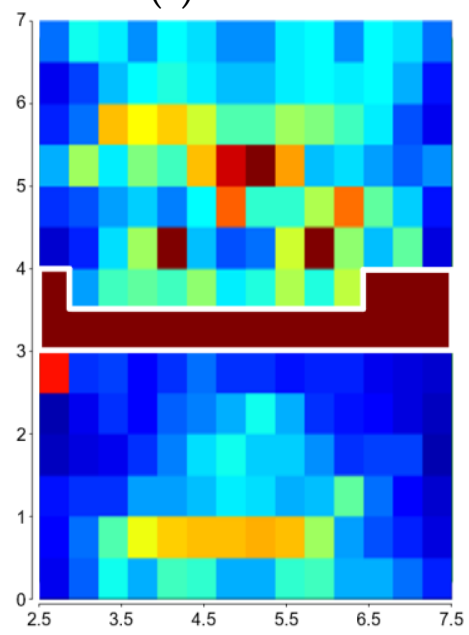

(f) $21 \times 20$

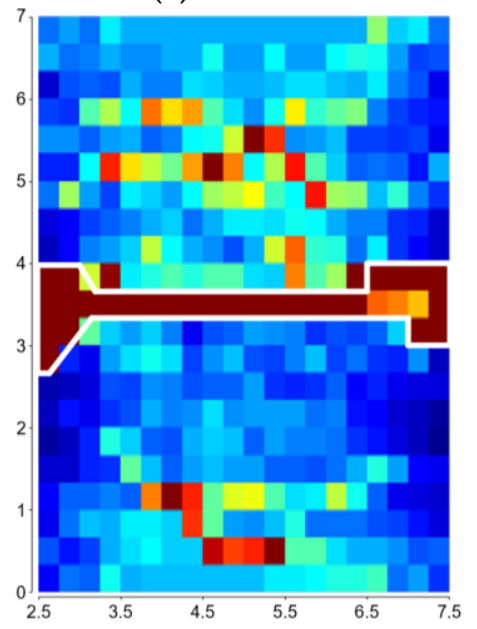

Figure 3.34. Diffusivity tomograms for SN profile by using SIRT-Cimmino algorithm under different resolutions. (a) The true distribution, $(\mathbf{b})-(\mathbf{f})$ the reconstruction under resolutions $14 \times 10,14 \times 15,21 \times 10,21 \times 15$, and $21 \times 20$, respectively. 
(a) $14 \times 10$

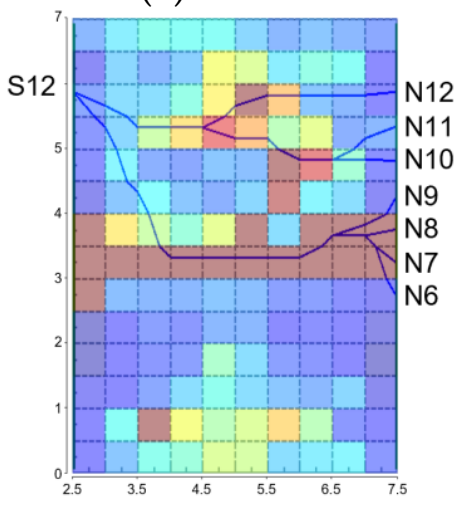

(b) $21 \times 10$

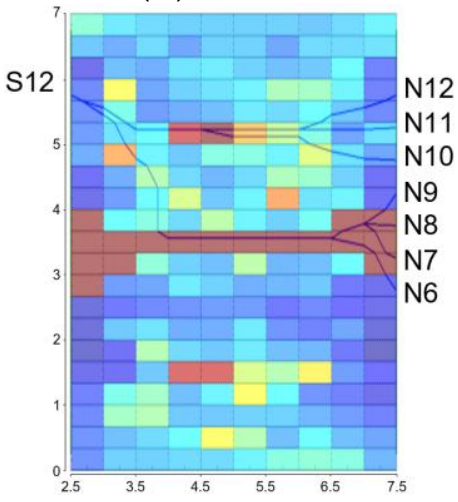

(c) $21 \times 20$

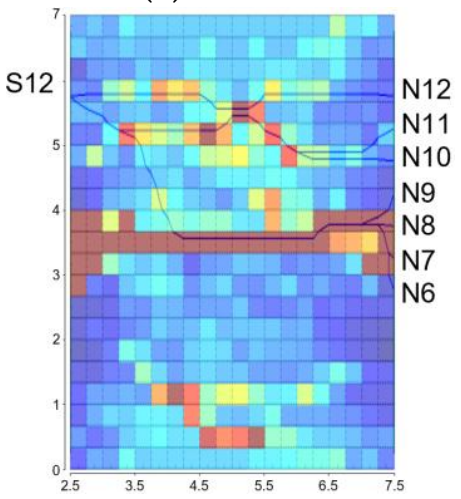

Figure 3.35. The trajectories from S12 to N6 - N12 in SN profile by using SIRTCimmino under different resolutions, (a) $14 \times 10$, (b) $21 \times 10$ and (c) $21 \times 20$.

\subsubsection{Conclusions and discussion}

The travel time based hydraulic tomography is investigated with an aquifer analog outcrop model. A dilemma is found during the application of SIRT algorithm. RMSE analysis suggests less iteration steps $(<10)$, while visual comparison indicates more iteration steps $(>15)$. Probable reasons might be the high sensitivity of the result to steps and the non-uniqueness of the solution.

SIRT-Cimmino algorithm is applied to compare the previous work. The evaluation shows that SIRT-Cimmino has a better performance than SIRT regarding structural similarity (i.e., feature reconstruction) and numerical accuracy.

High resolution distributions are tested by using SIRT-Cimmino. However, we do not see any improvement in the reconstruction of the primary feature - the high-D continuum. This could be attributed to three factors:

(a) The high non-uniqueness under high resolutions.

(b) Under high resolution, the height of the cell is smaller than half of the width of the continuum, the signal thus has at least two paths due to the property that signal prefers to traverse through high- $D$ cells. It is difficult 
to place identical number of trajectories into two paths, therefore one path could become the main path and the other is downgraded to moderate diffusivity layer. After iterations, the reconstructed continuum becomes thinner compared to the true distribution, for instance, in Figure 3.34 (d), (e) and (f).

(c) The distance between two wells are doubled by abandoning the central well, the travel times near the high- $D$ continuum are still very small. Even with the help of mesh refinement and short timestep in simulation software, the accuracy of the travel time computation is not promised. The travel times are too close to distinguish. This problem might lead to a slight vertical shift of the high- $D$ continuum, for example, in Figure 3.31 (e), the right part of the high- $D$ continuum is shifted upwards "one-cell-unit".

RMSE between the true distribution and reconstructed distributions with respect to the number of cells are computed in both profile studies, (Figure 3.36 and Figure 3.37). RMSE increases slightly as the number of cells increases. Does this result indicate the presence of error under high resolutions? To answer this question, more tests under different resolutions but with the same number of cells should be performed.

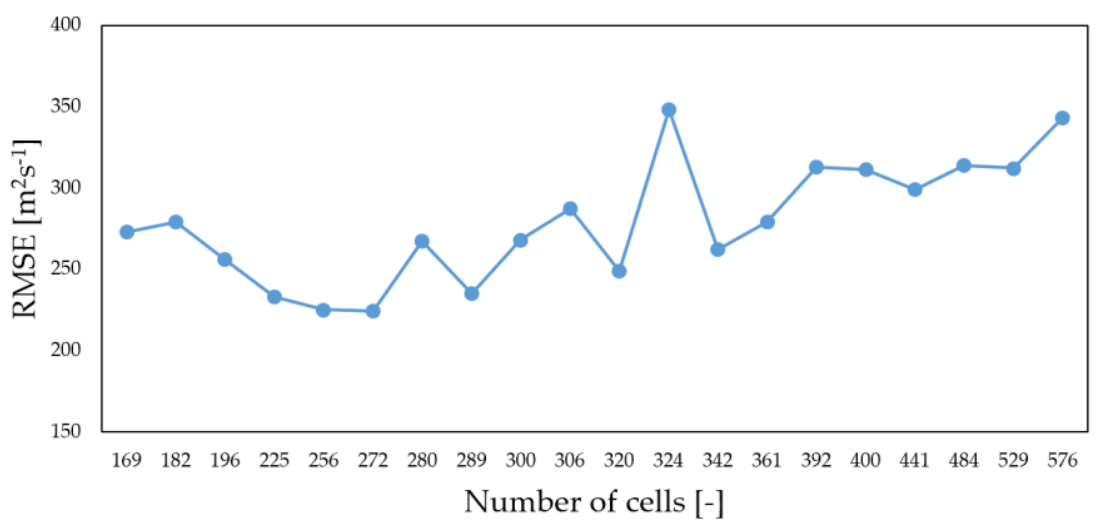

Figure 3.36. The RMSE with respect to the number of cells in the inversion for WE profile. 


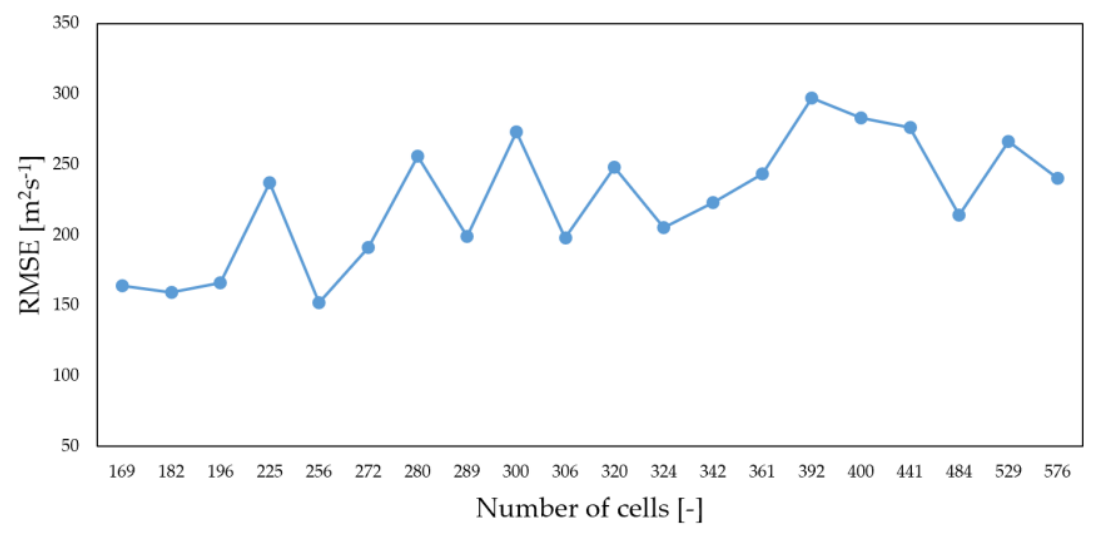

Figure 3.37. The RMSE with respect to the number of cells in the inversion for SN profile.

The visualization shows that WE profile is better reconstructed compared to SN profile. Two profiles have similar structures except the dip angle of the high$D$ continuum. Does the SIRT-Cimmino algorithm perform better in an inclined structure than in a horizontal structure? This hypothesis should be considered in future research. 


\section{Field Application}

\subsection{The Test Site}

\subsubsection{Test site description}

The test site is located at the north campus of University of Göttingen, Germany, near the Faculty of Geoscience and Geography. The test site is surrounded by a parking lot in the east, Robert-Koch-Straße in the west and Goldschmidtstraße in the north (Figure 4.1). A narrow stream (Lutter stream) is passing around 95 meters south of the site. A small pond, on the east side, and a building, on the southeast side, are located near the test area.

The site area is about $25 \mathrm{~m}^{2}$ and has six wells labeled as $\mathrm{O}, \mathrm{W}, \mathrm{S}, \mathrm{N}, \mathrm{M}$, and BHE (Figure 4.1). The first five wells are named by their locations $(\mathrm{O}, \mathrm{W}, \mathrm{S}, \mathrm{N}$, and $\mathrm{M}$ stand for east, west, south, north, and middle, respectively) and well BHE stands for geothermal borehole heat exchanger. Well BHE was drilled together with well W in 2008, while the other wells were drilled in 2012.

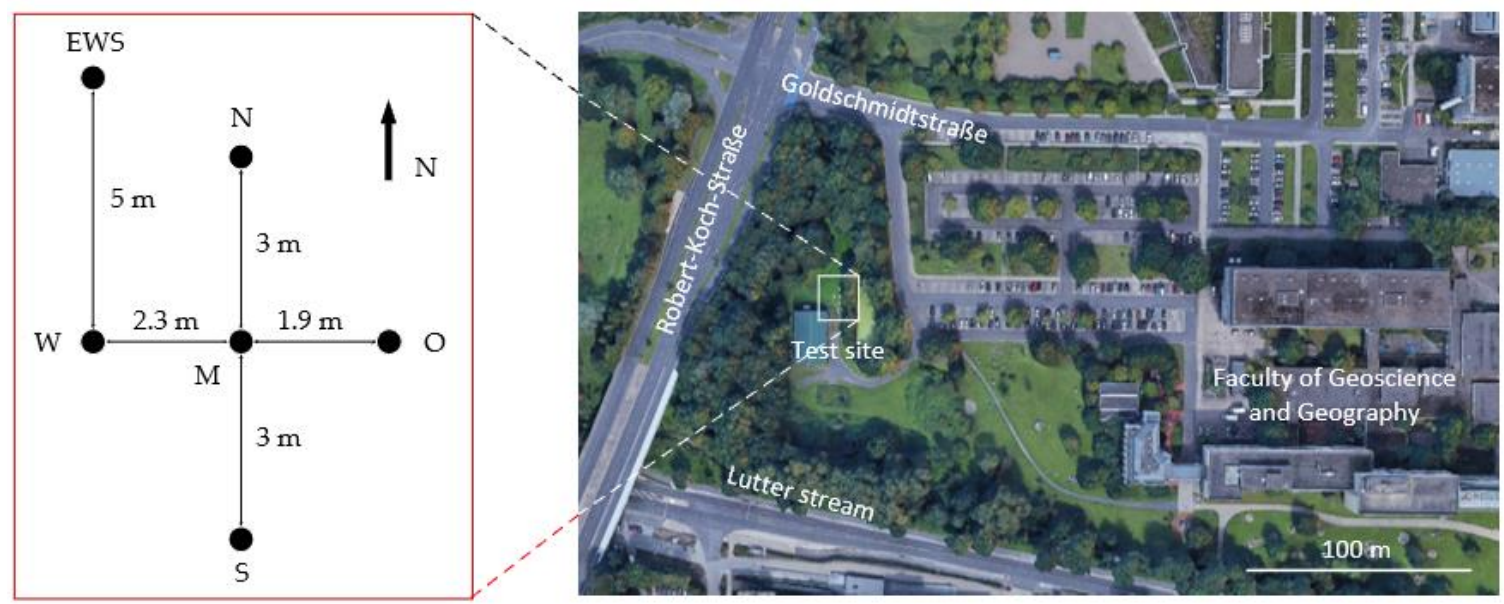

Figure 4.1. The arrangement of the wells (left), and the location of the test site (source: google map)

Wells $(\mathrm{O}, \mathrm{W}, \mathrm{S}, \mathrm{N}$, and $\mathrm{M}$ ) are $78 \mathrm{~m}$ deep and have similar construction (Figure 4.2). The well has 9 slotted PE pipe sections (i.e., screen, length each $5 \mathrm{~m}$, diameter $7.6 \mathrm{~cm}$ ) and 9 full cased PE pipe section (length each $3 \mathrm{~m}$, diameter $7.6 \mathrm{~cm}$ ) 
assembled in alternating order. We number the screens from the surface to the bottom. That is, in well $\mathrm{O}, \mathrm{O} 1$ is the first screen (close to the surface), and $\mathrm{O} 9$ is the ninth screen (at the bottom). A $6 \mathrm{~m}$ long and $4.5 \mathrm{~cm}$ thick high permeable gravel pack overlaps each screen section and fills up the space between each screen and well wall. A $2 \mathrm{~m}$ long and $4.5 \mathrm{~cm}$ thick high dense clay filling with low permeability takes up the space between each fully cased section and well wall. Due to this construction design, the well is hydraulically connected to different geological strata (Oberdorfer et al. 2013). 


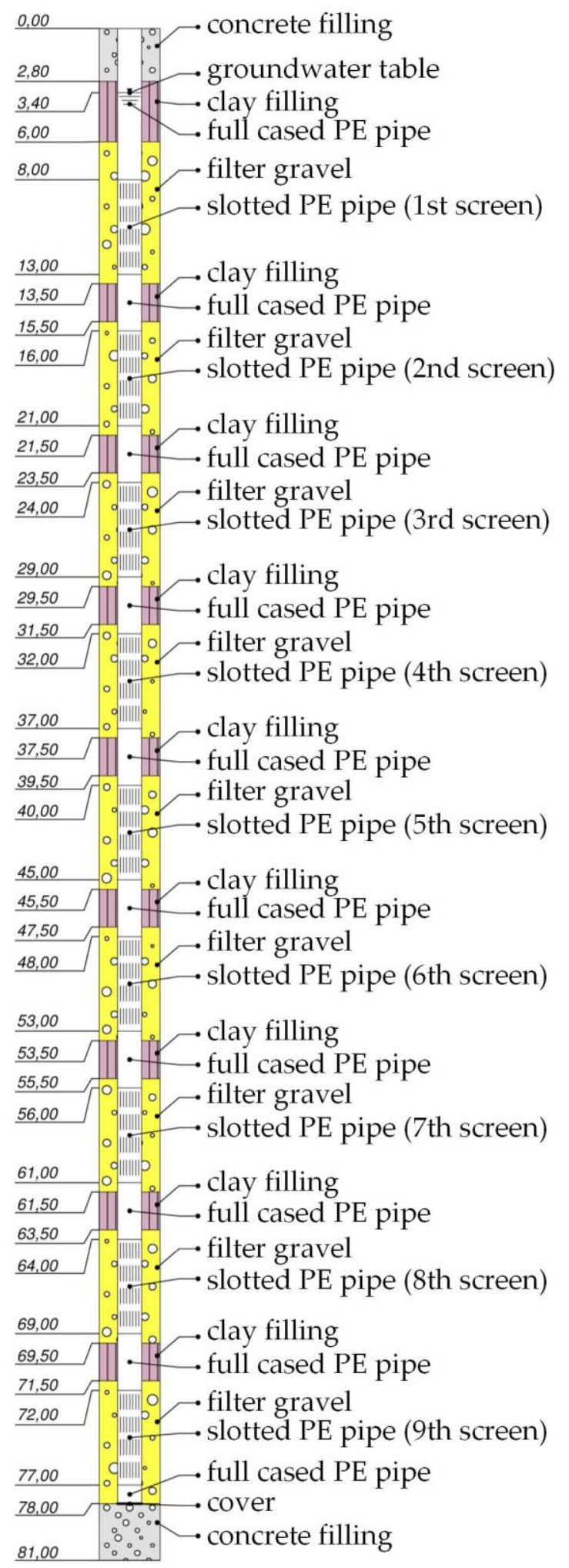

Figure 4.2. Sketch of the well construction. All five wells $\mathrm{O}, \mathrm{W}, \mathrm{S}, \mathrm{N}$, and $\mathrm{M}$ are constructed equally in this way (modified from Piecha 2008, Oberdorfer et al. 2013). 


\subsubsection{Previous research on this test site}

Various geophysical measurements, thermal and hydraulic experiments are conducted since constructing the test site.

LIAG measured geophysical characteristics of well $\mathrm{O}, \mathrm{W}, \mathrm{S}, \mathrm{N}$ and $\mathrm{M}$ using acoustic/optical televiewer, spectral gamma ray, vertical deflection, etc. The acoustic and optical scanning combined with gamma ray logs indicated a good correlation between well $\mathrm{O}, \mathrm{W}, \mathrm{N}$ and $\mathrm{M}$ at 35 to $70 \mathrm{~m}$ depth. Based on their results, Werner (2013) suggested a bedding plane with a dip angle of $75^{\circ}$ towards the south-east. The correlation can also be explained as layer duplication or other fault processes. Vertical deflection measurement showed that wells are deflected from their original location on the surface with the depth increment (Figure 4.3). The vertical deflection is an important factor and should be considered alongside other parameters in future field data evaluation. The azimuth of calcite veins indicated strong changes in geological parameters at the core scale which indicates heterogeneity of hydraulic parameters. 

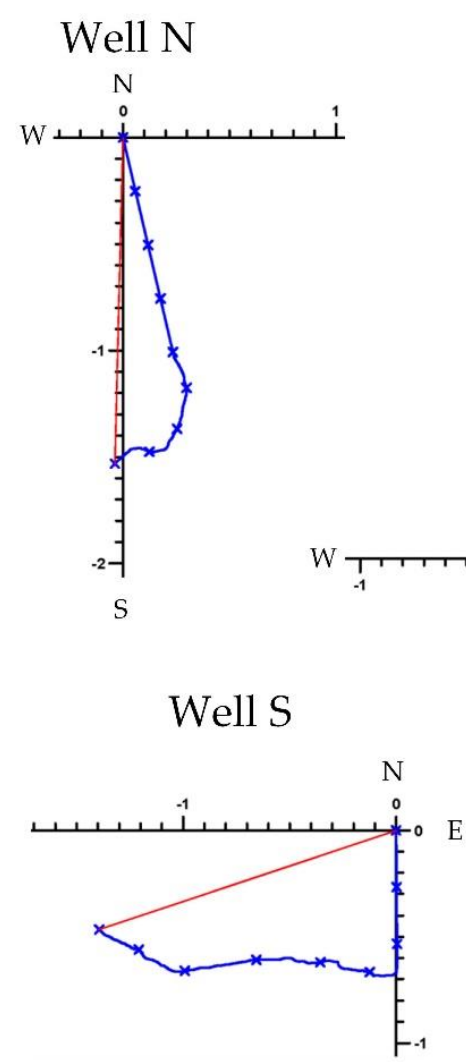

Well O
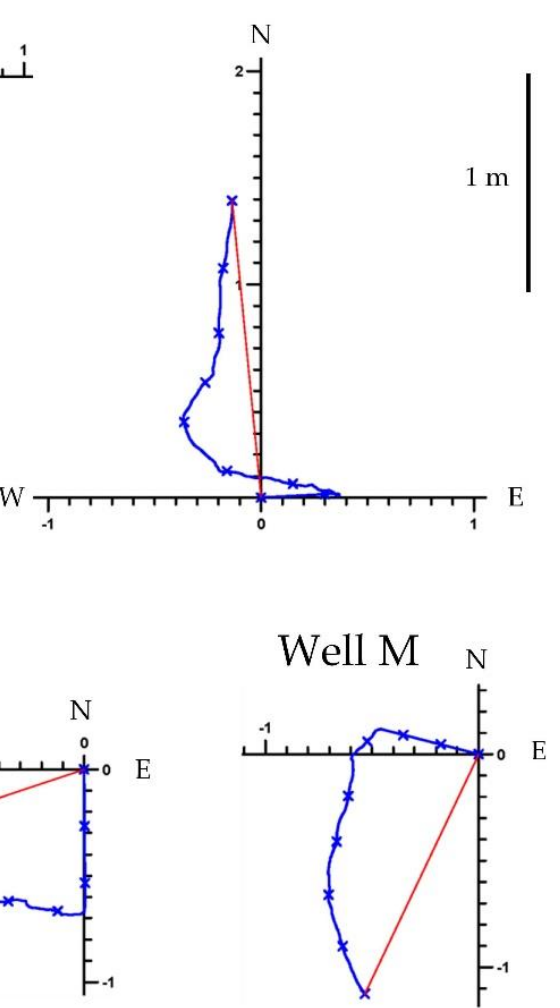

Figure 4.3. The vertical deflection of wells N, O, S, and M (Werner 2013).

Schuster (2015) measured the texture and fracture of the drilling core materials from well $\mathrm{N}$ and performed laboratory experiments to derive the hydraulic conductivity and anisotropies of the permeability. The hydraulic conductivities range from $1 \times 10^{-6} \mathrm{~m} \cdot \mathrm{s}^{-1}$ and $1 \times 10^{-10} \mathrm{~m} \cdot \mathrm{s}^{-1}$ and strongly depend on the texture and fracture geometry of the core sample material. In addition, he stressed that the result gave a qualitative understanding in a small scale and can not be transferred to a larger scale.

Piecha (2008) performed a thermal response test to investigate the thermal conductivity of the site. He derived a depth-oriented thermal conductivity profile between injection well (W) and observation well (BHE). The thermal conductivity ranges between 1.62 and $1.95 \mathrm{Wm}^{-1} \mathrm{~K}^{-1}$ and indicates a general thermal homogeneity. The author declared 2.5\% measurements errors, and their results showed a slightly higher thermal conductivity at $42 \leqslant$ depth $\leqslant 47 \mathrm{~m}$. In addition, 
Piecha modeled a number of scenarios with assuming different fracture widths $(0.1,1,3$, and $5 \mathrm{~cm})$ and hydraulic conductivities $\left(7.23 \times 10^{-5}, 7.23 \times 10^{-3}, 6.57 \times 10^{-}\right.$ ${ }^{2}$, and $1.8 \times 10^{-1} \mathrm{~m} \cdot \mathrm{s}^{-1}$ ) to study their influence on the heat transport. They suggested that the fracture width has a significant influence on heat transport when it is larger than $3 \mathrm{~cm}$.

Bätzel (2017) performed a thermal tracer test to study the aquifer heterogeneity since the construction of wells $\mathrm{O}, \mathrm{S}, \mathrm{N}$, and M in 2012. The warm water was injected in well $\mathrm{M}$ and the temperatures in wells $\mathrm{O}, \mathrm{W}, \mathrm{S}$ and $\mathrm{N}$ were measured. After 15 days, a packer was installed at $17 \mathrm{~m}$ depth in injection well to force heat tracer to move through the deep layers. After 17 days, the packer was moved to depth of $15 \mathrm{~m}$. The derived temperature profile in the observation wells verified the aquifer heterogeneity of the test site and proved the existence of highly conductive fractures. In the temperature profile of well $\mathrm{O}$ (Figure 4.4), three quick response are captured at depth of 12, 18 and $27 \mathrm{~m}$ within screens $\mathrm{O} 1, \mathrm{O} 2$, and $\mathrm{O} 3$, respectively. After the installation of the pack at $15 \mathrm{~m}$, the response at depth of 12 $\mathrm{m}$ declines while the responses at depth of 18 and $27 \mathrm{~m}$ intensify. The absent response implies a good thermal connection between the part of well M (above 15 $\mathrm{m}$ ) and the screen O1. Besides, the result revealed a better thermal connection in West-East direction compared to the North-South direction. 


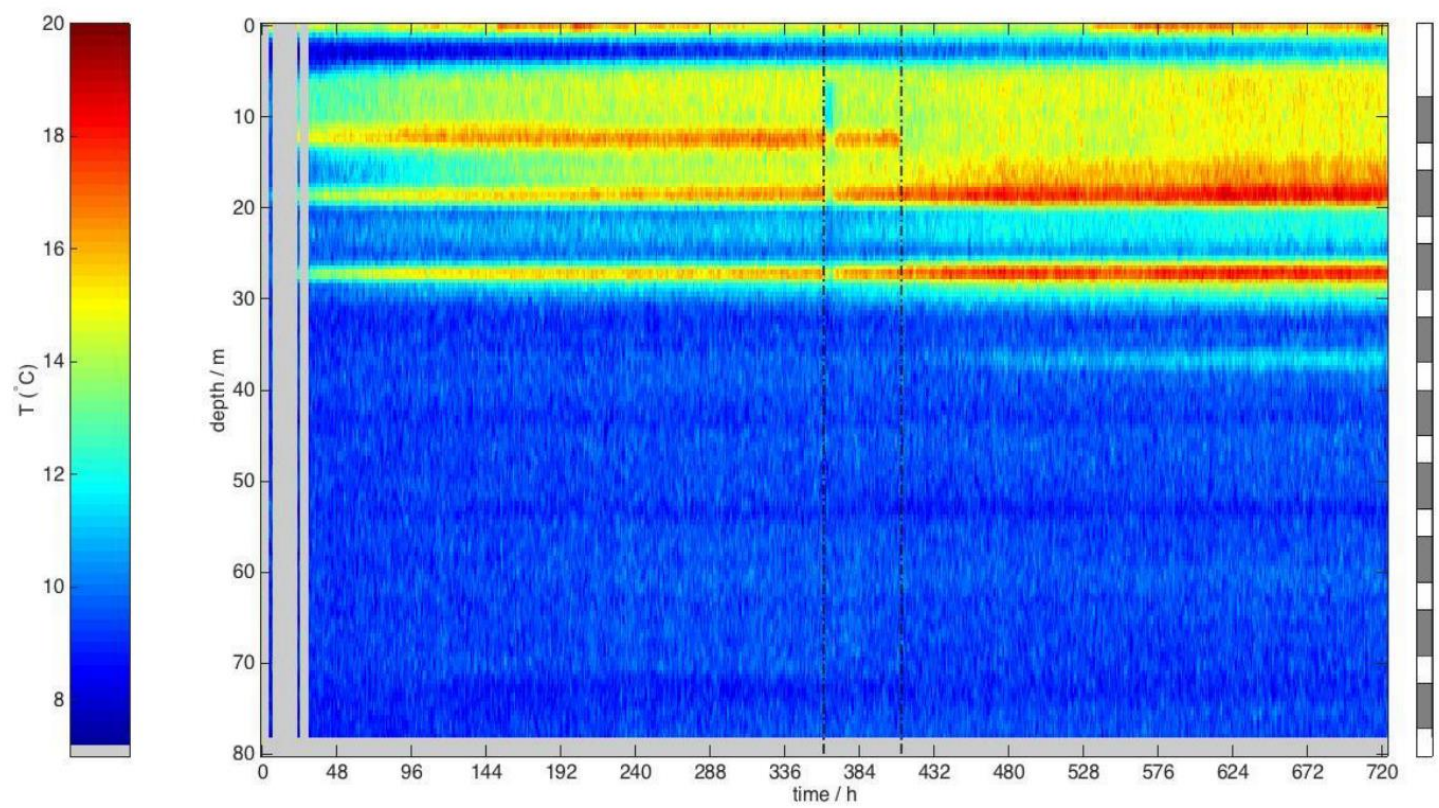

Figure 4.4. Temperature development in well O. Dash lines at 360 and $408 \mathrm{~h}$ mark the installation of the packer at the depth of 17 and $15 \mathrm{~m}$, respectively. The bar on the right side represents the filter (grey) and full pipe sections (white) (after Bätzel 2017).

Oberdorfer et al. (2013) presented the well configuration of the five well cluster $(\mathrm{O}, \mathrm{W}, \mathrm{S}, \mathrm{N}$, and $\mathrm{M})$ and performed cross-well multi-level pumping tests at the first screen interval of each well. The installation and feature of the cross-well multi-well pumping test will be introduced in the next subsection. They analyzed the data using Theis method (Theis 1935) and Cooper \& Jacob method (Cooper and Jacob 1946) and estimate the hydraulic conductivity $K$ and specific storage $S_{s .}$ Table 4.1 shows that $K$ values are almost similar in all wells, while $S_{s}$ near well O is significantly smaller compared to the other wells. The result suggests the presence of a highly fractured aquifer close to well $\mathrm{O}$.

Table 4.1. Result of cross-well pumping test at the first screen of five wells (Oberdorfer et al. 2013).

\begin{tabular}{cccccc}
\hline & $\mathrm{N}$ & $\mathrm{N}$ & $\mathrm{O}$ & $\mathrm{S}$ & $\mathrm{W}$ \\
\hline$K\left[\mathrm{~m} \cdot \mathrm{s}^{-1}\right]$ & $3 \times 10^{-4}$ & $5 \times 10^{-4}$ & $3 \times 10^{-4}$ & $4 \times 10^{-4}$ & $5 \times 10^{-4}$ \\
$S_{s}\left[\mathrm{~m}^{-1}\right]$ & & $4 \times 10^{-4}$ & $2 \times 10^{-6}$ & $1 \times 10^{-3}$ & $6 \times 10^{-4}$ \\
\hline
\end{tabular}


Shrestha (2013), Wen (2014) and Tan (2015) performed cross-well integral pumping tests and cross-well multi-level pumping tests. Integral pumping tests were conducted in wells $\mathrm{O}, \mathrm{W}, \mathrm{S}, \mathrm{N}$ and $\mathrm{M}$. One well was pumped, and the drawdown was observed in four neighboring wells. The pumping lasted 3 to 4 hours and the drawdown was recorded by pressure transducers. They considered the aquifer is confined and applied Cooper \& Jacob straight-line method (Cooper and Jacob 1946) to match the early time drawdown (10s - $100 \mathrm{~s})$ and late time drawdown (after $1000 \mathrm{~s}$ ). The evaluated hydraulic conductivities were $4.5 \times 10^{-5}$ $\mathrm{m} \cdot \mathrm{s}^{-1}$ (early time) and $7.6 \times 10^{-5} \mathrm{~m} \cdot \mathrm{s}^{-1}$ (late time). The estimated storativity was $2.2 \times$ $10^{-3}$ (early time) and $1.1 \times 10^{-5}$ (late time).

Cross-well multi-level pumping tests were carried out in two directions: North-South and West-East. Double packer system was used for both pumping and observation wells to isolate the screen section at the same depth. Cooper \& Jacob (Cooper and Jacob 1946) and Theis / Hantush solution (Theis 1935, Hantush 1961a) were utilized for matching the drawdown. For convenience, the aquifer area between $i$ th screens is notated as i-i layer. Shrestha (2013) indicates, the hydraulic conductivity and the specific storage do not vary largely to show a significant difference in principle, however, it is sufficient to represent the heterogeneities of the subsurface. Based on the result, the 9-9 layer in North-South direction is properly connected than the other layers or is closer to the fracture.

Travel times based hydraulic tomography inversion was performed by using geophysical software GeoTomCG. According to the reconstructed diffusivity distribution, Wen (2014) and Tan (2015) demonstrated the presence of a high diffusivity zone between $36 \mathrm{~m}$ and $63 \mathrm{~m}$ depth in the North-South direction.

The above-mentioned geophysical measurements and thermal response tests indicated the existence and complex of heterogeneity of the test site. The analytical solution used in Oberdorfer et al. (2013) is not suitable for a heterogenous aquifer 
and perhaps leads to a misestimation. Based on the schematic diagram of the packer system in Shrestha (2013), Wen (2014) and Tan (2015), the screen is not isolated, since the packer system $(3.4 \mathrm{~m})$ is $1.6 \mathrm{~m}$ shorter than the screen $(5 \mathrm{~m})$. A reliable characterization of the heterogeneity for the test site is needed.

\subsection{Pumping Tests}

Wells $\mathrm{O}$ and $\mathrm{M}$ serve as pumping well and observation well, respectively. Due to the equipment limitation, only the aquifer between wells $\mathrm{M}$ and $\mathrm{O}$ from the 2nd screen to the 5th screen was investigated (Figure 4.5). Fully penetrating (integral) cross-well pumping tests were first conducted to estimate the hydraulic conductivity and specific storage. To investigate the vertical variation of hydraulic parameters, we performed a series of cross-well multi-well short-term pumping tests that are partially penetrating. In this profile, 16 cross-well multi-level pumping tests were designed. Each test is notated by the screens where pumping and observation take place. For instance, the pumping test with pumping section $\mathrm{O} 4$ and observation section $\mathrm{M} 3$ is notated as $\mathrm{O} 4 \mathrm{M} 3$.

To isolate the screen in the filter gravel section, an inflatable double packer system was used in pumping and observation wells (Figure 4.6). The inflated packers were placed above and below the filter gravel section to stop the vertical flow in the well. 


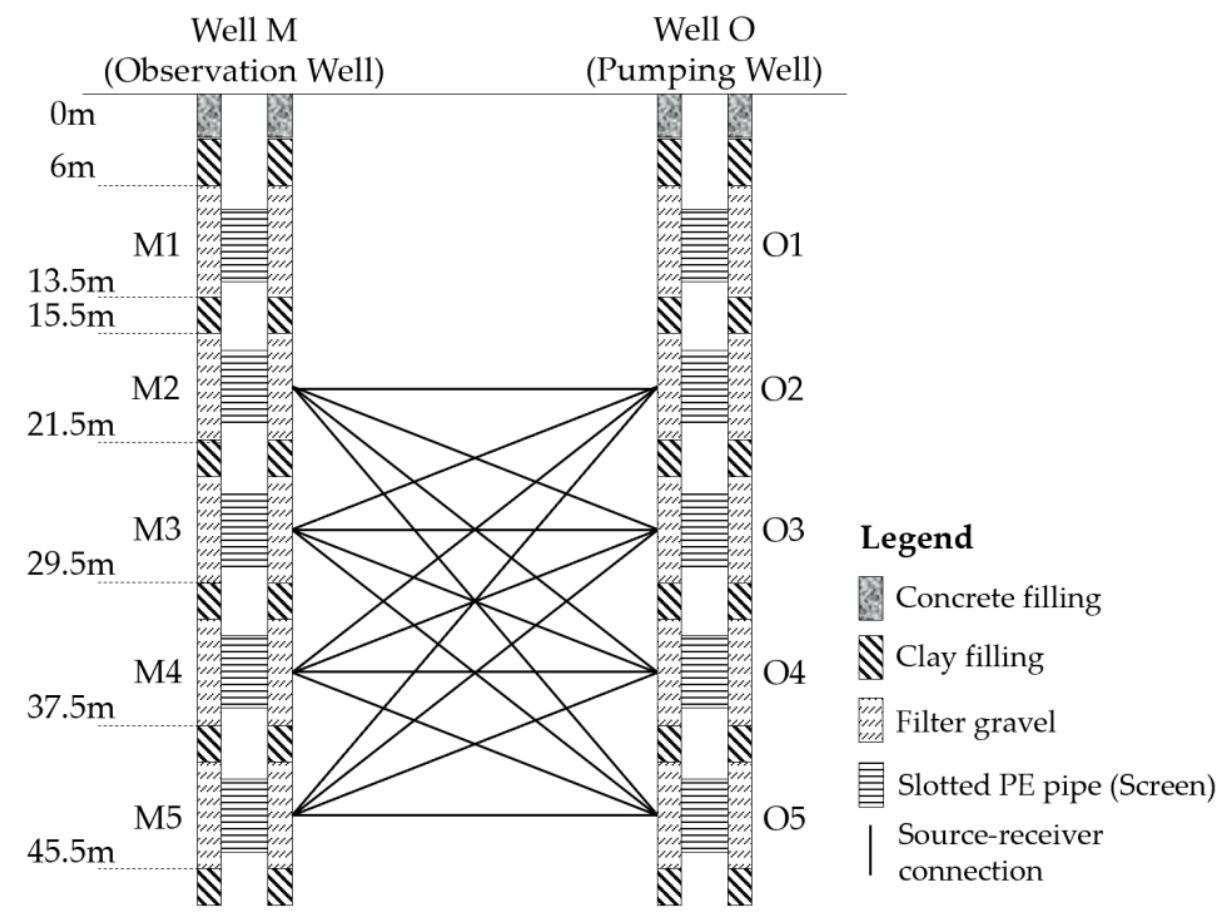

Figure 4.5. Sketch of the cross-well multi-level short term pumping tests. Note that the source-receiver connections are not the real flow path, they demonstrate the configuration of sources (right endpoint of the connection, as the pumping point) and receivers (left endpoint of the connection, as the observation point). This sketch is modified from Yang (2019).

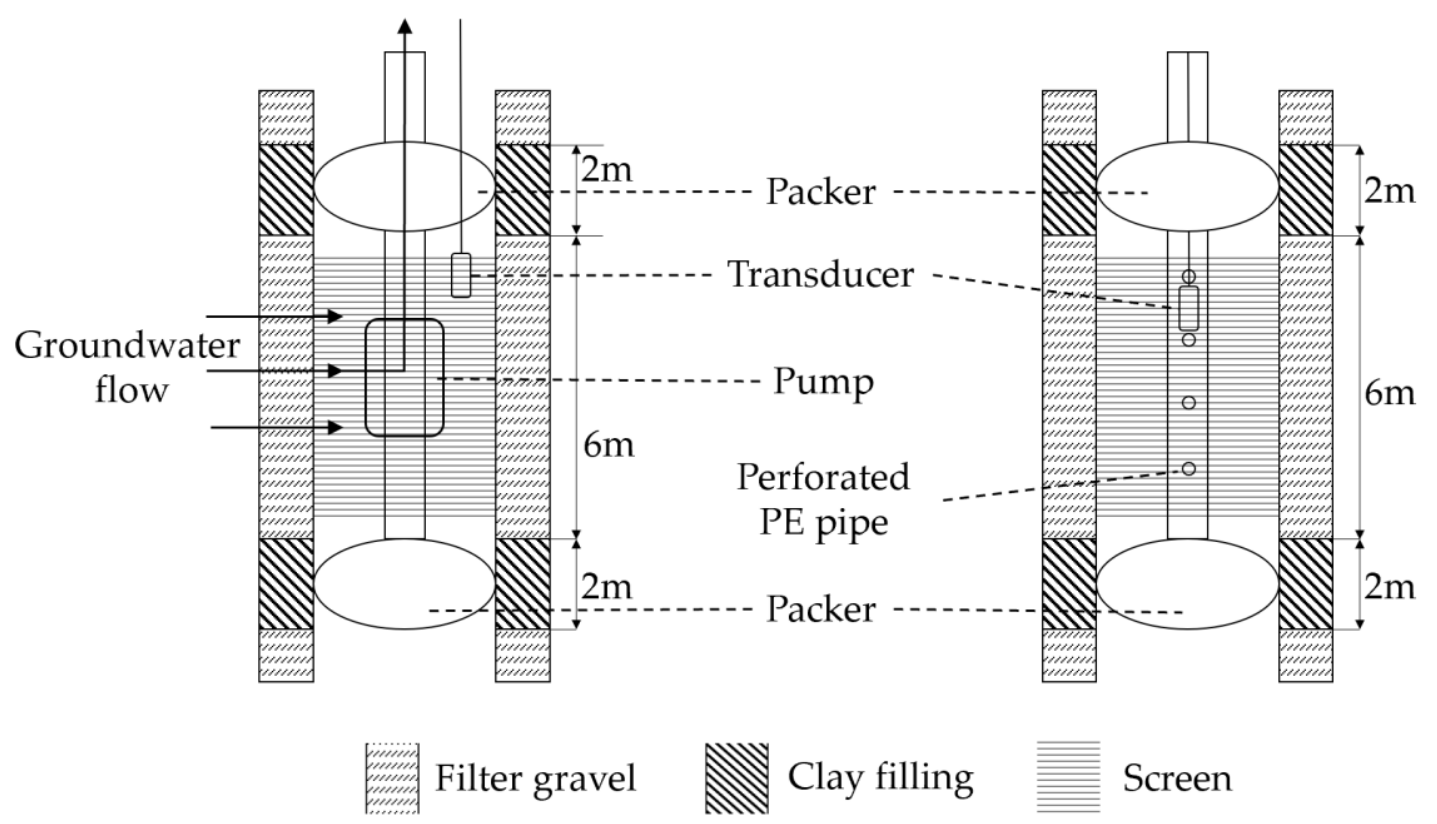

Figure 4.6. Sketch of double packer system in pumping well (left) and observation well (right). 
The groundwater is withdrawn by a submersible pump (Grundfos MP1) with a flow rate up to $40 \mathrm{~L} \cdot \mathrm{min}^{-1}$. A frequency controller is used to adjust the flow rate to produce the highest drawdown (at various depths) to reduce the relative noise. Transducer PDCR 1830 (GE) records the drawdown in well O while transducer PDCR 35/D (GE) and Diver (vanEssen Micro-Diver) were placed in well M. PDRC 35/D is synchronized with PDCR 1830 through the datalogger (Campbell Scientific CR3000 Micrologger) to provide data with sufficient accuracy. Diver works individually and has a high noise resistance. The combined utilization of these three transducers compromises the reliability of the measurement. The pumping and recovery periods of $\mathrm{O} 2 \mathrm{M} 2, \mathrm{O} 3 \mathrm{M} 3, \mathrm{O} 4 \mathrm{M} 4$, and $\mathrm{O} 5 \mathrm{M} 5$ tests last 30 and 90 minutes, respectively. In other tests, the pumping and recovery periods last 5 and 45 minutes, respectively.

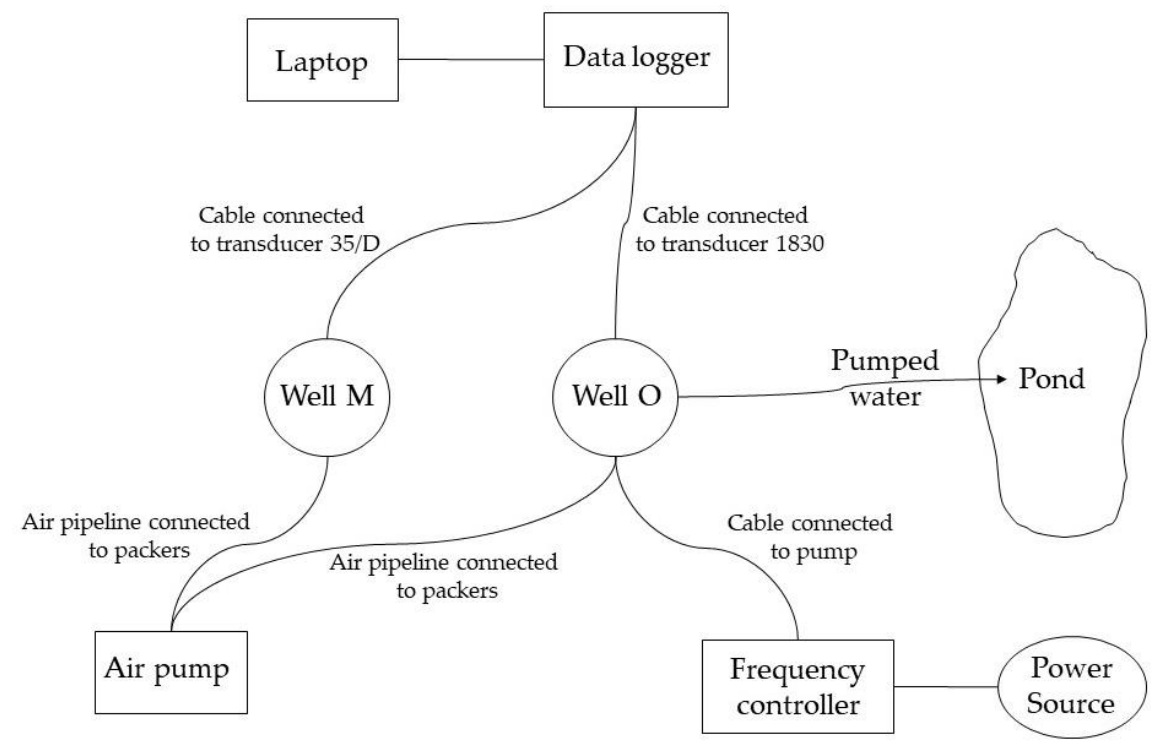

Figure 4.7. Schematic of pumping test set-up. Transducer Diver is not shown in the schematic (modified from Yang (2019)). 


\subsection{Application of Analytical Methods}

\subsubsection{Diagnostic plot}

Figure 4.8 shows the diagnostic plot of drawdown in O4 (from test O4M4). In the early time, the drawdown and the derivative roughly follow the same straight line with unit slope. This behavior indicates the wellbore storage effect and excludes the existence of vertical fracture with infinite conductivity. The delayed response, from 6th to 20th second (shown in the red square), is most likely from the instantaneous dewatering of gravel pack. The horizontal derivative between 100th and 2000th second implies the presence of a homogenous area. The derivative becomes zero at the end of the experiment, but the pumping time is too short to identify the boundary type. However, the boundary condition is not necessary for tomography inversion. The combination of the intermediate time behavior and the tendency towards zero suggests the presence of a leaky aquifer. Therefore, unconfined, leaky and (fractured) double porosity models are suitable models for parameter estimation.

The same analysis is applied to examine the drawdown data at $\mathrm{O} 2, \mathrm{O} 3$, and O5. Wellbore storage and skin effect during the early time are found in each screen. Horizontal drawdown and decreased derivative are detected in the late time. The bilinear flow (in fractures) can be identified by the slope of drawdown during the intermediate time at $\mathrm{O} 2$ and $\mathrm{O} 3$. The diagnostic results combined with the previous geological survey suggest that fractures are the most pronounced feature of the studied area. The following type curve matching analysis applies (fractured) double porosity model for all tests. 


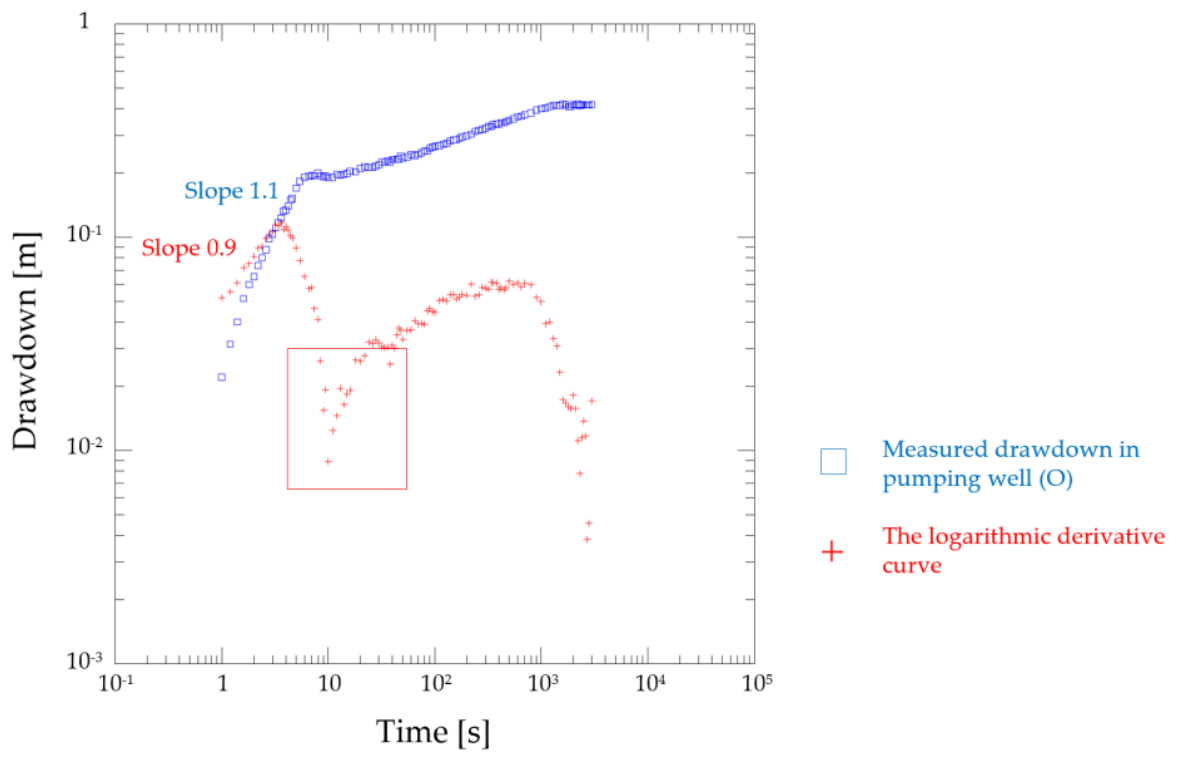

Figure 4.8. The diagnostic plot for the test O4M4 by using Aqtesolv. The blue squares are the measured drawdown in the pumping well $(\mathrm{O})$, and the red crosses are the logarithmic derivative by using Bourdet differentiation method (Bourdet et al. 1989).

\subsubsection{Type curve analysis}

Due to the test conditions, i.e., partial penetrating wells, wellbore storage, and skin effect, Moench (fractured) double porosity solution is utilized to fit the drawdown in each test. This analytical solution was developed by Moench (1997) and describes the unsteady flow to a finite-diameter well in an isotropic fractured aquifer assuming a double-porosity model. Figure 4.9 shows the simultaneous fitting of drawdown in pumping and observation wells. Four main parameters were estimated: fracture conductivity $K f$, fracture specific storage $S_{s m}$, matrix conductivity $\mathrm{Km}$ and matrix specific storage $S_{s m}$. Two values were introduced based on parameters: $K f / K m$ (the ratio of $K f$ and $K m$ ) and $D f$ (fracture diffusivity, the ratio of $K f$ and $\left.S_{s f}\right) . K f / K m$ scales the heterogeneity of the aquifer and characterizes the density of the fracture (Warren and Root 1963). $D f$ is directly 
related to travel time, because the fractures, as the primary porosity, are highly interconnected and contribute the main transport in the model.

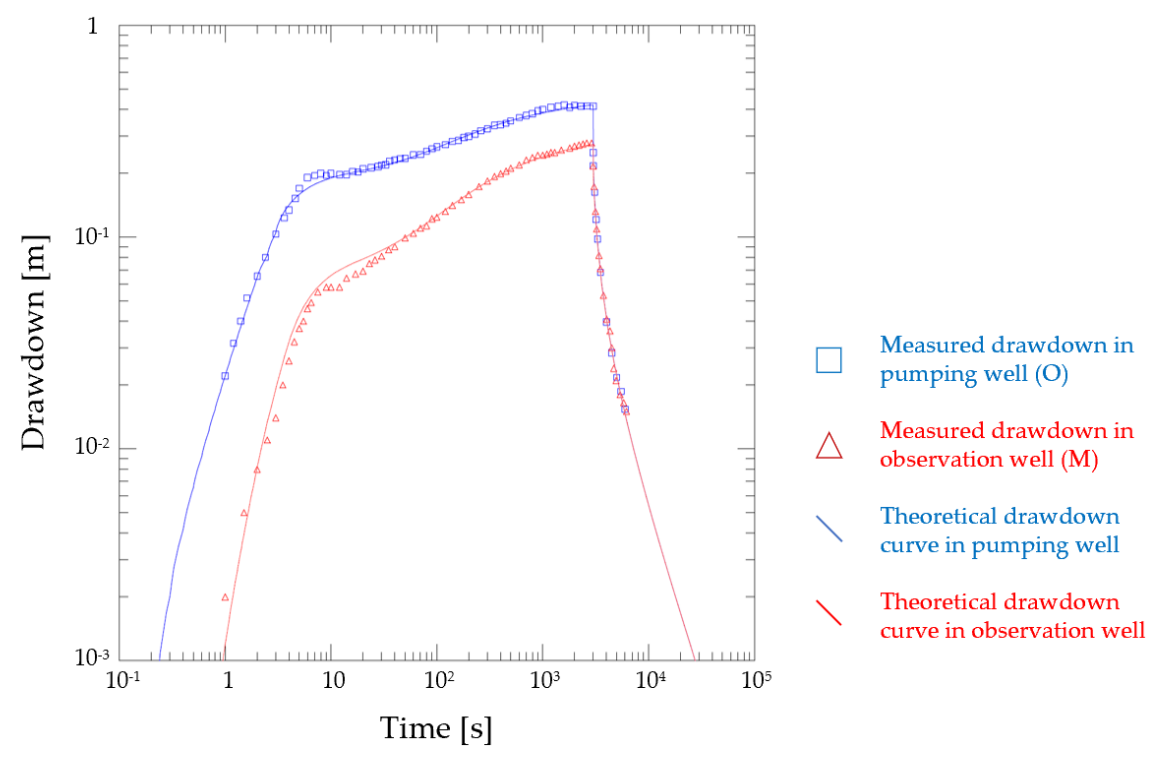

Figure 4.9. Type curve matching for the test $\mathrm{O} 4 \mathrm{M} 4$ by using Moench (fractured) double porosity solution (software: Aqtesolv).

Three kinds of tests are analyzed: cross-well integral test, cross-well multilevel pumping test, and single-well multi-level pumping test. The result of the integral test is shown in Table 4.2 and reveals the general information of the aquifer. The fracture conductivity $K f$ and specific storage $S_{s} f$ are close to the values evaluated through early time drawdown (10 - 100s) in the work of Shrestha (2013), Wen (2014) and Tan (2015). The evaluated $D f$ can be used to verify the diffusivity obtained from the travel times and substantiate the initial values for the inversion.

Thirteen cross-well multi-level pumping tests were successfully conducted, and the results are listed in Table 4.3. It is found that all $\mathrm{Kf}$ have the same order of magnitude. The ranges of $K f$ and $K m$ are consistent with Schuster (2015). Based on $\mathrm{K} / \mathrm{Km}$, the upper half of the investigation area has a high fracture density, while the area between $\mathrm{O} 5$ and M5 has a low fracture density. Furthermore, the order of magnitude of the ratio implies that the fractures in the upper half of the area strongly perturb flow and carry as much of it as the matrix (Matthäi and Belayneh 
2004). The range of the estimated diffusivity $\left(0.2-270 \mathrm{~m}^{2} \mathrm{~s}^{-1}\right)$ helps us to determine the min and max constraints of the inversion.

We use the drawdown of the pumping well as a single well test to estimate the parameters in the close proximity. As shown in Table 4.4, $\mathrm{Kf}$ and $\mathrm{Km}$ are much larger than those in Table 4.2 and Table 4.3, while $\mathrm{Kf} / \mathrm{Km}$ near O2, $\mathrm{O} 3$ and $\mathrm{O} 4$ are relatively smaller. The diffusivity values are not available since the specific storage can not be determined by the drawdown of the pumping well individually (Wu et al. 2005).

Table 4.2. The result of type curve matching for the cross-well integral pumping test.

\begin{tabular}{ccccccc}
\hline & $K f$ & $S_{s} f$ & $K m$ & $S_{s} m$ & $K f / K m$ & $D f$ \\
\hline & {$\left[\mathrm{m}^{-1} \mathrm{~s}^{-1}\right]$} & {$\left[\mathrm{m}^{-1}\right]$} & {$\left[\mathrm{m}^{-1} \mathrm{~s}^{-1}\right]$} & {$\left[\mathrm{m}^{-1}\right]$} & {$[-]$} & {$\left[\mathrm{m}^{2} \mathrm{~s}^{-1}\right]$} \\
\hline integral & $7.5 \times 10^{-5}$ & $1.0 \times 10^{-6}$ & $6.5 \times 10^{-9}$ & $5.1 \times 10^{-3}$ & $1.2 \times 10^{4}$ & $7.5 \times 10^{1}$ \\
\hline
\end{tabular}

Table 4.3. The result of type curve matching for the cross-well multi-level pumping tests.

\begin{tabular}{ccccccc}
\hline & $\mathrm{Kf}$ & $S_{s} f$ & $\mathrm{Km}$ & $S_{s m}$ & $\mathrm{Kf} / \mathrm{Km}$ & $\mathrm{Df}$ \\
\hline & {$\left[\mathrm{m} \cdot \mathrm{s}^{-1}\right]$} & {$\left[\mathrm{m}^{-1}\right]$} & {$\left[{\left.\mathrm{m} \cdot \mathrm{s}^{-1}\right]}^{-5}\right.$} & {$\left[\mathrm{m}^{-1}\right]$} & {$[-]$} & {$\left[\mathrm{m}^{2} \mathrm{~s}^{-1}\right]$} \\
\hline O2M2 & $5.5 \times 10^{-5}$ & $5.4 \times 10^{-6}$ & $4.0 \times 10^{-9}$ & $1.6 \times 10^{-3}$ & $1.4 \times 10^{4}$ & $1.0 \times 10^{1}$ \\
\hline O2M3 & $5.2 \times 10^{-5}$ & $1.1 \times 10^{-6}$ & $3.7 \times 10^{-9}$ & $1.5 \times 10^{-3}$ & $1.4 \times 10^{4}$ & $4.7 \times 10^{1}$ \\
\hline O2M4 & $4.6 \times 10^{-5}$ & $3.0 \times 10^{-6}$ & $7.1 \times 10^{-10}$ & $1.4 \times 10^{-3}$ & $6.5 \times 10^{4}$ & $1.5 \times 10^{1}$ \\
\hline O3M2 & $4.1 \times 10^{-5}$ & $5.8 \times 10^{-6}$ & $6.0 \times 10^{-9}$ & $1.5 \times 10^{-3}$ & $6.8 \times 10^{3}$ & $7.1 \times 10^{0}$ \\
\hline O3M3 & $5.7 \times 10^{-5}$ & $2.9 \times 10^{-6}$ & $2.7 \times 10^{-8}$ & $1.7 \times 10^{-3}$ & $2.1 \times 10^{3}$ & $2.0 \times 10^{1}$ \\
\hline O3M4 & $5.2 \times 10^{-5}$ & $3.2 \times 10^{-6}$ & $7.5 \times 10^{-10}$ & $2.1 \times 10^{-3}$ & $6.9 \times 10^{4}$ & $1.6 \times 10^{1}$ \\
\hline O4M2 & $4.2 \times 10^{-5}$ & $2.7 \times 10^{-6}$ & $4.2 \times 10^{-10}$ & $1.9 \times 10^{-3}$ & $1.0 \times 10^{5}$ & $1.6 \times 10^{1}$ \\
\hline O4M3 & $5.1 \times 10^{-5}$ & $1.9 \times 10^{-7}$ & $2.7 \times 10^{-9}$ & $2.5 \times 10^{-3}$ & $1.9 \times 10^{4}$ & $2.7 \times 10^{2}$ \\
\hline O4M4 & $5.3 \times 10^{-5}$ & $2.5 \times 10^{-4}$ & $8.6 \times 10^{-7}$ & $2.2 \times 10^{-3}$ & $6.2 \times 10^{1}$ & $2.1 \times 10^{-1}$ \\
\hline O5M2 & $6.7 \times 10^{-5}$ & $1.0 \times 10^{-6}$ & $1.5 \times 10^{-9}$ & $3.7 \times 10^{-3}$ & $4.5 \times 10^{4}$ & $6.7 \times 10^{1}$ \\
\hline O5M3 & $2.3 \times 10^{-5}$ & $1.3 \times 10^{-4}$ & $9.6 \times 10^{-8}$ & $6.1 \times 10^{-4}$ & $2.4 \times 10^{2}$ & $1.8 \times 10^{-1}$ \\
\hline O5M4 & $3.1 \times 10^{-5}$ & $1.8 \times 10^{-4}$ & $3.9 \times 10^{-7}$ & $1.0 \times 10^{-3}$ & $7.9 \times 10^{1}$ & $1.7 \times 10^{-1}$ \\
\hline O5M5 & $5.1 \times 10^{-5}$ & $1.6 \times 10^{-5}$ & $7.7 \times 10^{-8}$ & $1.2 \times 10^{-3}$ & $6.6 \times 10^{2}$ & $3.2 \times 10^{0}$ \\
\hline & & & & & &
\end{tabular}


Table 4.4. The result of type curve matching for the single-well multi-level pumping tests.

\begin{tabular}{cccc}
\hline & $\mathrm{Kf}$ & $\mathrm{Km}$ & $\mathrm{Kf} / \mathrm{Km}$ \\
\hline & {$\left[\mathrm{m} \cdot \mathrm{s}^{-1}\right]$} & {$\left[\mathrm{m} \cdot \mathrm{s}^{-1}\right]$} & {$[-]$} \\
\hline O2 & $2.0 \times 10^{-4}$ & $1.3 \times 10^{-6}$ & $1.5 \times 10^{2}$ \\
\hline O3 & $1.2 \times 10^{-4}$ & $1.2 \times 10^{-6}$ & $1.0 \times 10^{2}$ \\
\hline O4 & $1.2 \times 10^{-4}$ & $4.7 \times 10^{-7}$ & $2.6 \times 10^{2}$ \\
\hline O5 & $1.1 \times 10^{-4}$ & $3.4 \times 10^{-7}$ & $3.2 \times 10^{2}$ \\
\hline
\end{tabular}

\subsection{Application of Travel Time Based Hydraulic Tomography}

\subsubsection{Data pre-processing}

In our field experiment, the main sources of the noise are the environment, vibration of the pump and electromagnetic induction. The environmental noise results from transportation, earth tide, weather condition, groundwater flow, etc. The pump vibration that propagates through the pipes disturbs the transducer measurement in the pumping well, furthermore, it propagates through the aquifer and affect the measurement accuracy in the observation well. The electromagnetic induction is generated by AC power cables connecting to electric driven devices and the controller switching the pumping rate. Our transducers measure the pressure by converting the mechanical pressure into an electrical signal, but this conversion process is sensitive to the electromagnetic induction. To reduce the noise, the experiments were carried out on cloudy days, unnecessary devices were turned off during the test, the controller was placed about 50 meters away from the site and power cables were hung in the air.

The drawdown during the pumping and recovery phases are monitored. The data of the pumping phase is visibly affected by noise (Figure 4.10). We use wavelet method and polynomial regression to remove the noise and rebuild the original drawdown. The methodology, application, and evaluation of the denoising process is reported in Yang (2019). 
In the recovery phase, the shutdown of the pump also generates a kind of Heaviside signal, and the hydraulic response is a minimum of the drawdown derivative (Figure 4.11). Once the pump and the controller are shutdown, the absence of vibration and electromagnetic induction gives a noiseless observation. Thus, we use the recovery phase to compute travel time to achieve desired accuracy. Because of the high permeability of the gravel pack, the observation well is first supplied by the water from the gravel pack and then from the aquifer. This feature results in a derivative with two minimums ( $t_{a}$ and $t_{b}$ in Figure 4.11). The travel time $t_{b}$ is difficult to be distinguished from $t_{a}$, when the source and receiver are closely located or hydraulically well connected. Herein, the source and receiver of the same depth are in close proximity (only 1.9 meters), and thus the corresponding travel time can not be derived from the recovery phase.

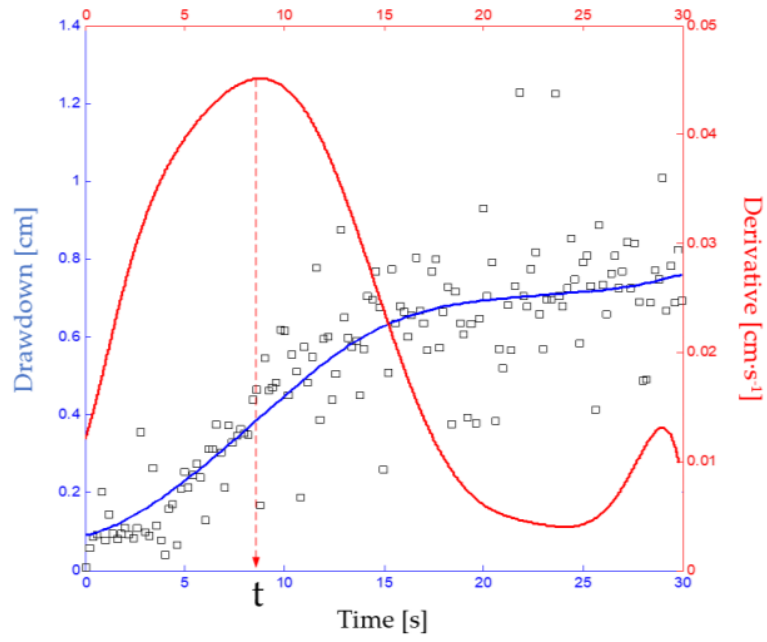

Measured drawdown in pumping phase Polynomial fitting after wavelet denoising First derivative of the polynomial

Figure 4.10. Travel time computation by using the drawdown in the pumping phase of the test O3M2. The black squares are the drawdown measured at M2, and the blue curve represents the data after wavelet denoising and polynomial regression. The red curve shows the first derivative of the polynomial (blue curve) and $t$ is the travel time. 


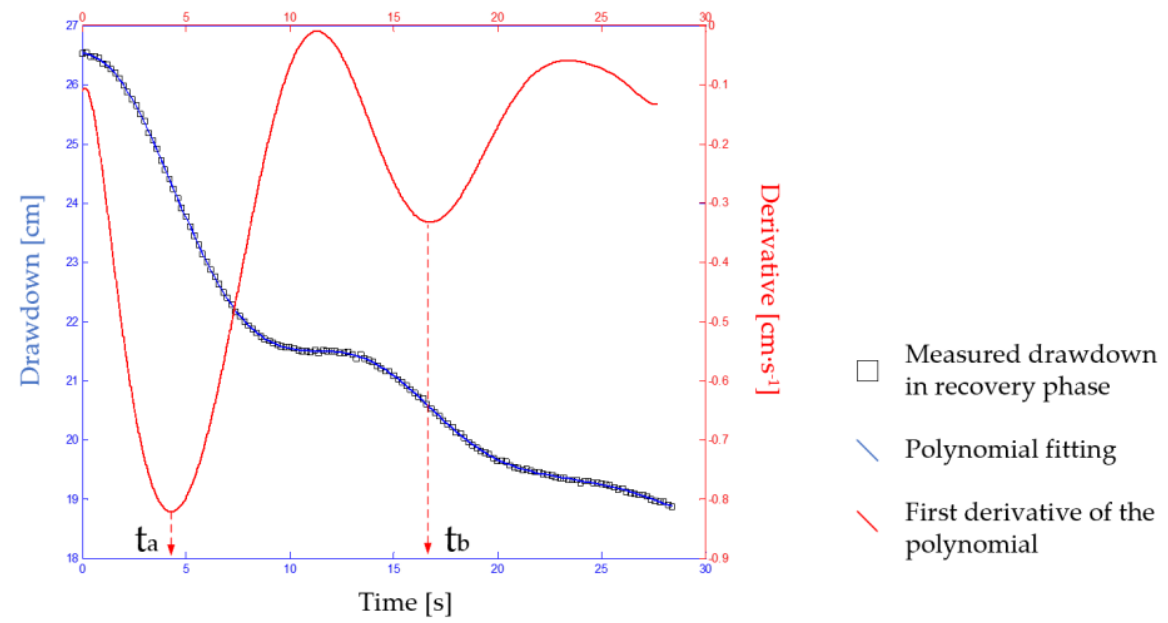

Figure 4.11. Travel time computation by using the drawdown in the recovery phase of the test O4M3. Compare to Figure 4.10, the measured drawdown (black squares) is less noisy, and wavelet denoising method was not utilized. Two minimums ( $t_{a}$ and $t_{b}$ ) are detected in the derivative (red curve), $t_{a}$ is caused by the water released from the fractures, while $t_{b}$ is the travel time of pressure response that propagates through the aquifer between two wells.

\subsubsection{Travel time obtainment}

Figure 4.12 shows the obtained travel times. Note that the locations of source and receiver pairs differ from those in the design (Figure 4.5). The modification is based on two reasons, (a) the pump can only be installed at the top of screen due to the equipment limitation in the practice, (b) the water in the borehole is assumed incompressible, the signal thus travels in the borehole with an infinite high speed and the travel time in the screen can be neglected. 
(a)

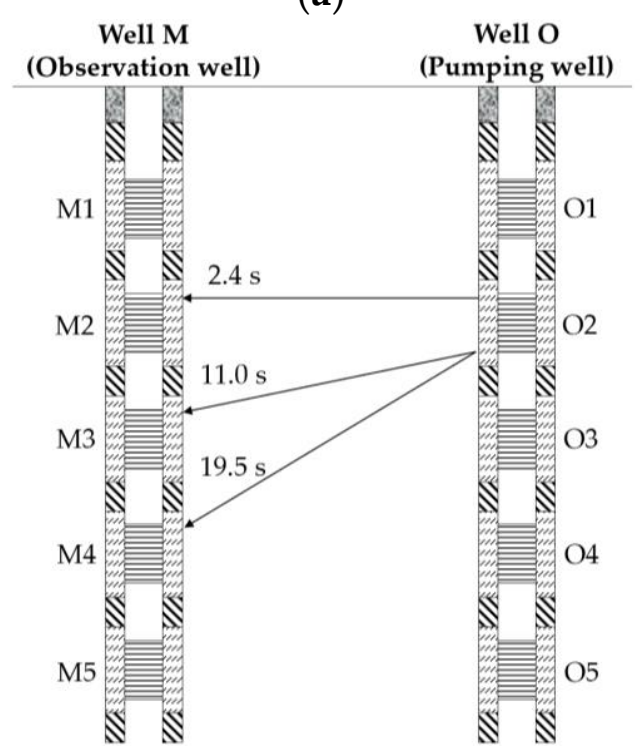

(c)

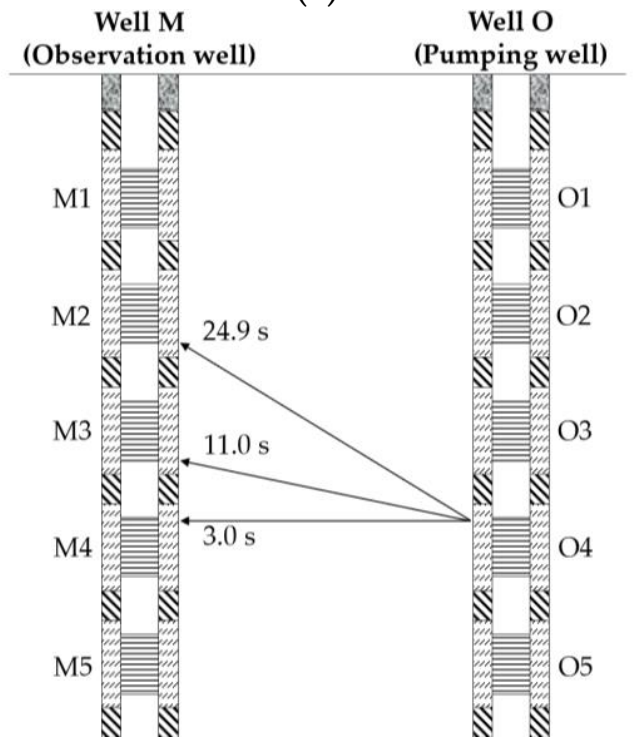

(b)

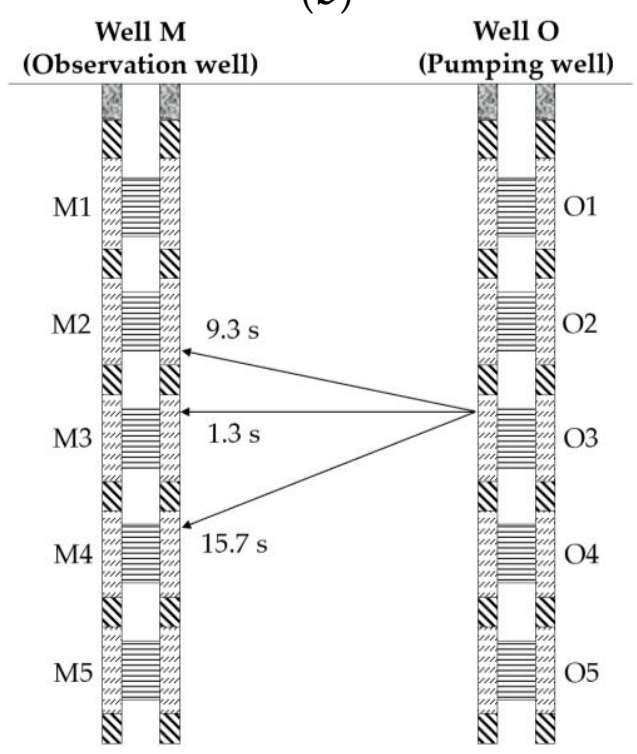

(d)

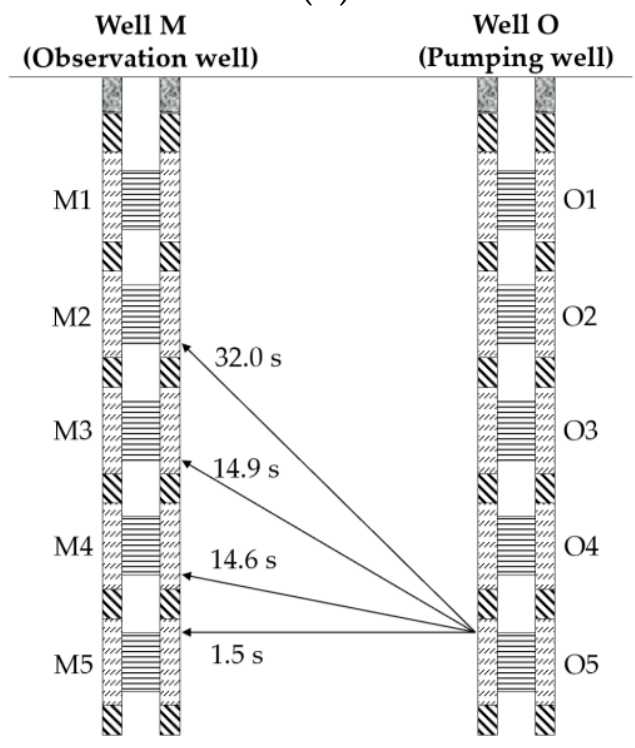

Figure 4.12. The obtained travel times map for the test site. Note that the locations of source and receiver pairs are changed (compared with Figure 4.5).

\subsubsection{Diffusivity reconstruction by using SIRT algorithm}

The inversion uses default initial value and min-constraint $\left(0.59 \mathrm{~m}^{2} \mathrm{~s}^{-1}\right.$ and $0.0059 \mathrm{~m}^{2} \mathrm{~s}^{-1}$, respectively). Considering the highest $\mathrm{Df}$ in Table 4.3, the maxconstraint (upper limit) is adjusted to $1000 \mathrm{~m}^{2} \mathrm{~s}^{-1}$, rather than $59 \mathrm{~m}^{2} \mathrm{~s}^{-1}$. Figure 4.13 shows the convergence of the residual for the first 50 steps under resolution $9 \times 6$. 
The tomograms at 8 th, 10th, 15th and 50th steps are illustrated in Figure 4.14. Each of these four distributions presents a horizontal high- $D$ layer at the middle depth. The layer connects to the bottom of $\mathrm{O} 3$ and occupies two thirds of the length between $\mathrm{O} 3$ and $\mathrm{M} 3$. Another feature is the high value near the bottom of $\mathrm{O} 2$. Every distribution has more than one cell reaching the upper limit.

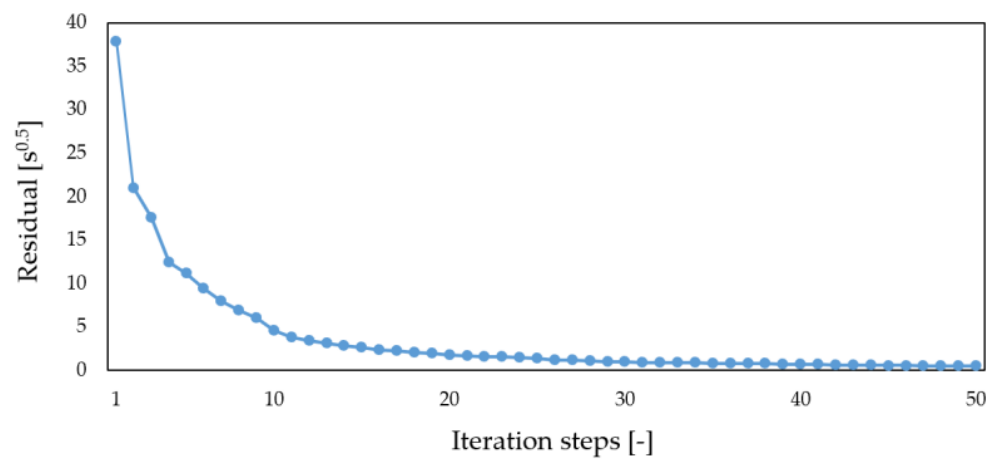

Figure 4.13. Residual of travel time for 50 iteration steps by using SIRT under $9 \times$ 6 resolution. 
(a) step 8

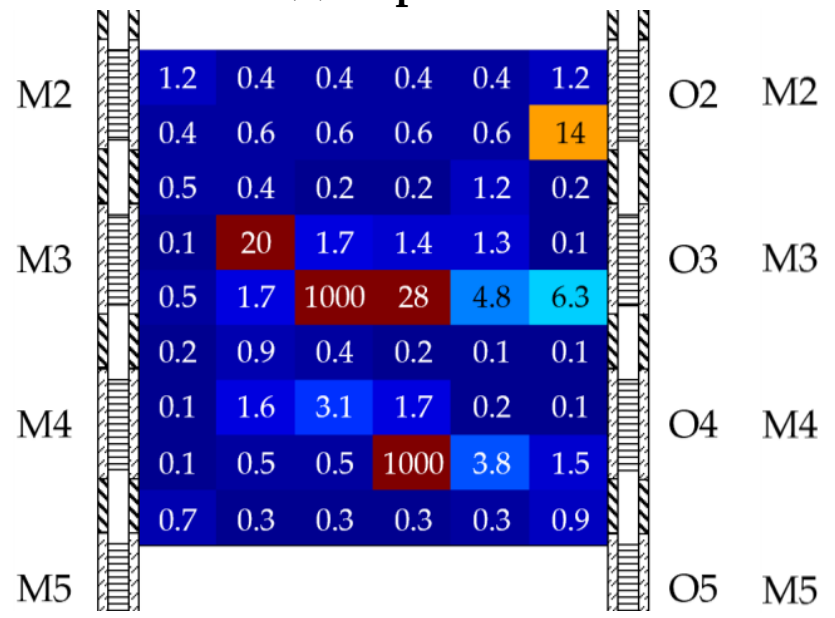

(c) step 15

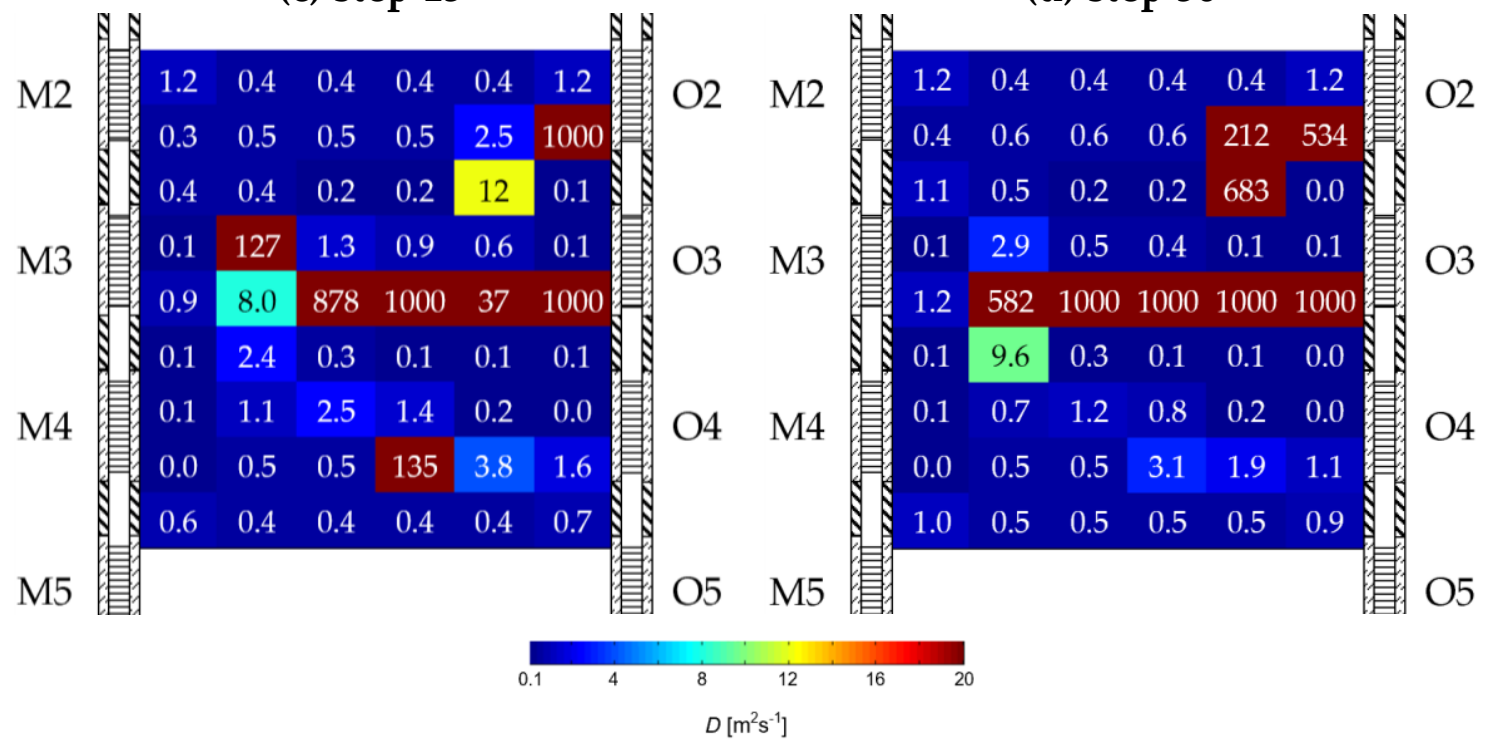

Figure 4.14. Diffusivity tomograms based on the inversion by using SIRT under $9 \times 6$ resolution at (a) 8, (b) 10, (c) 15, and (d) 50 steps, respectively.

\subsubsection{Diffusivity reconstruction by using SIRT-Cimmino algorithm}

The initial setting is the same as the inversion with SIRT. Figure 4.15 shows the residual for the first 50 steps under resolution $9 \times 6$. According to the result selection rule, the distribution at 50th step is considered the inversion result. The features are similar, but the upper limit is not reached. The inversion results under resolutions $9 \times 8$ and $9 \times 9$ are exhibited in Figure 4.17. Note that a clear high $D$ continuum is detected between $\mathrm{O} 2$ and M2. 


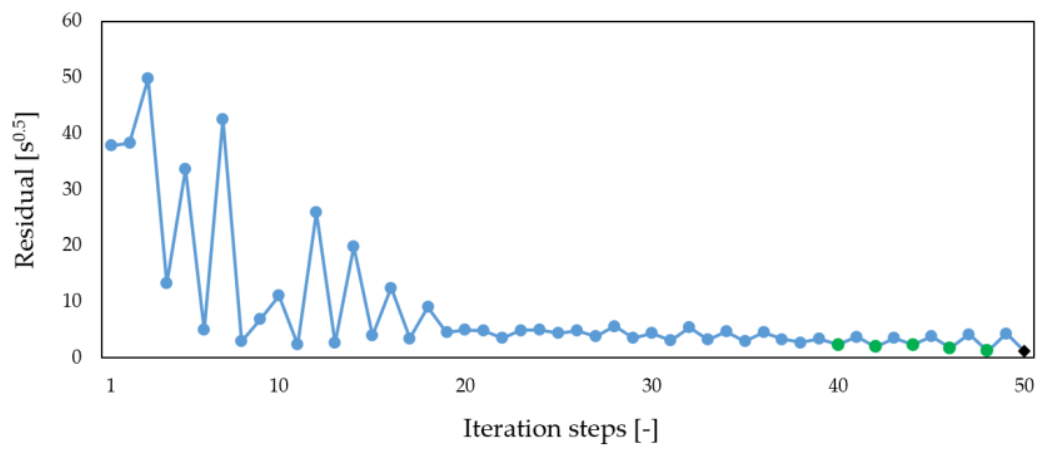

Figure 4.15. Residual of travel time for 50 iteration steps by using SIRT-Cimmino under $9 \times 6$ resolution.

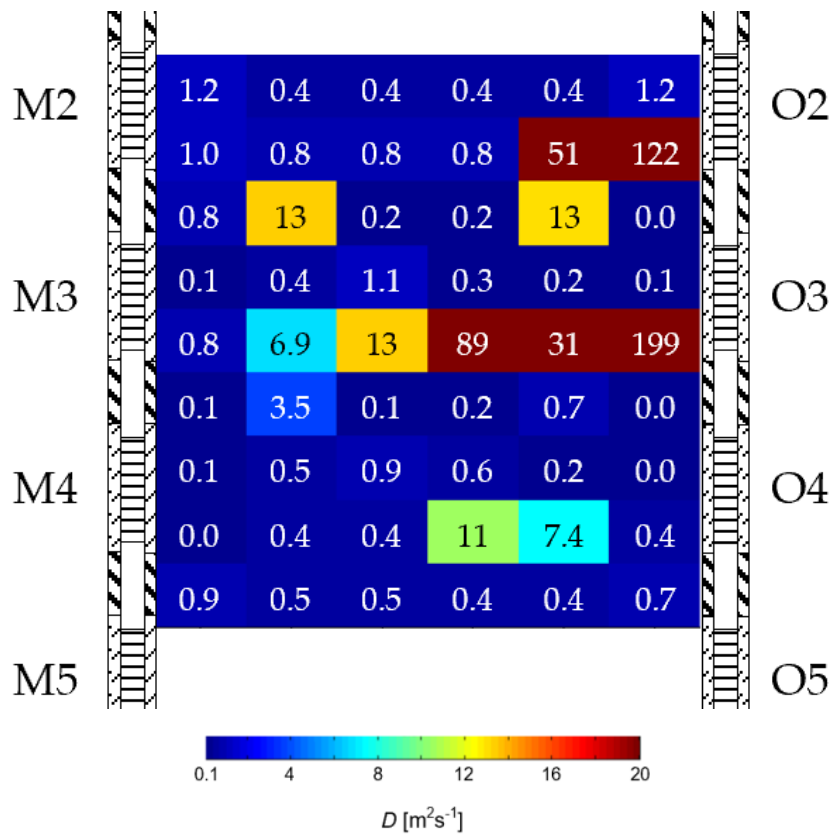

Figure 4.16. Inversion result by using SIRT Cimmino under resolution $9 \times 6$. 


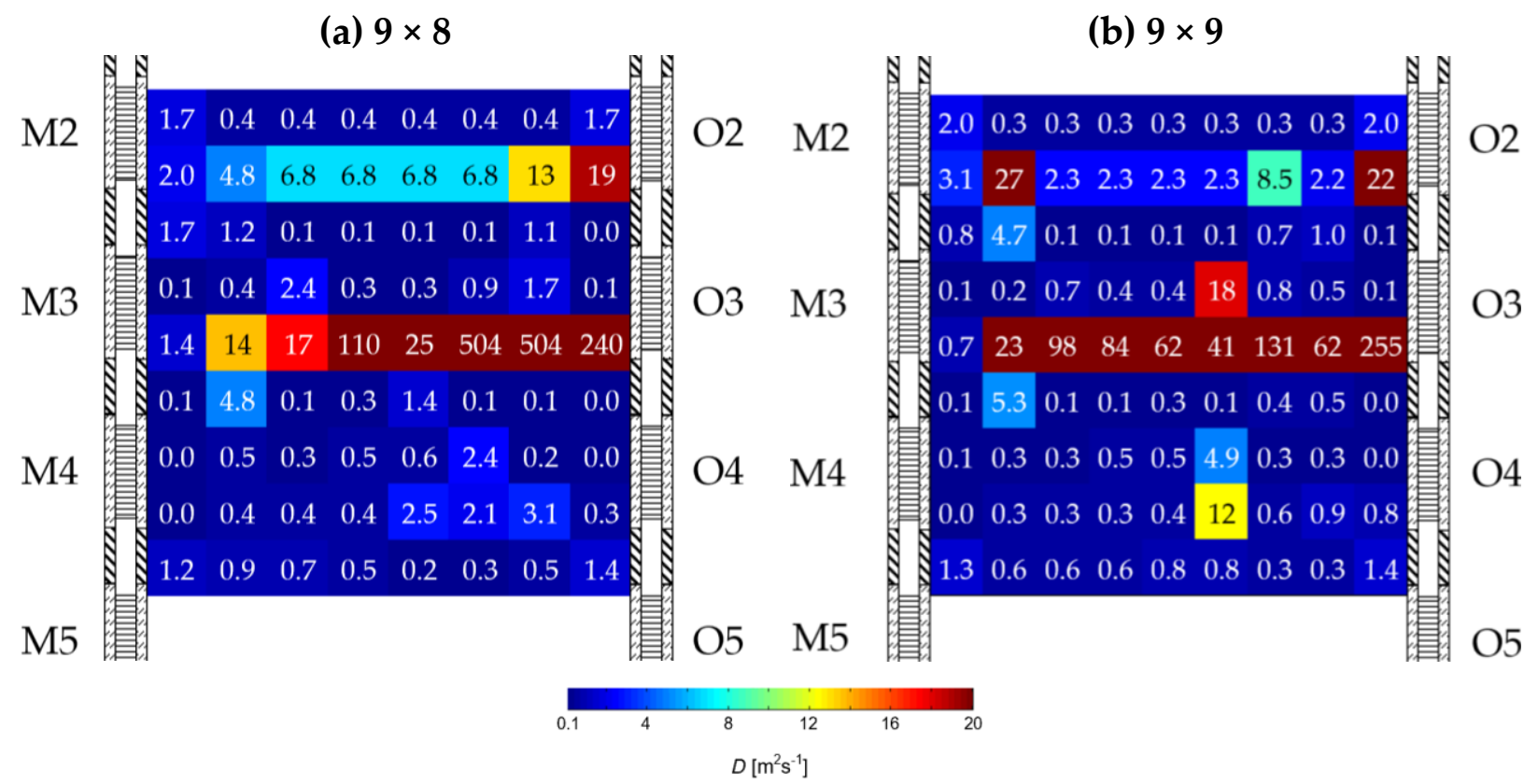

Figure 4.17. Inversion result by using SIRT Cimmino (a) under resolution $9 \times 8$ and $(\mathbf{b})$ under resolution $9 \times 9$.

\subsection{Result Evaluation}

We compare the inversion results with the type curve matching analysis and previous research. First of all, the most pronounced feature in all inversion results is a high- $D$ layer in the middle depth of the investigation area, where the type curve matching analysis gives high $D f$ (see tests $\mathrm{O} 4 \mathrm{M} 3, \mathrm{O} 3 \mathrm{M} 3$ and O3M4). Two inversion results in Figure 4.17 reconstruct a secondary high- $D$ continuum connecting $\mathrm{O} 2$ and M2. This feature can be explained by the slight large $D f$ in test O2M3. The positions of both high-D zones are consistent with the result of the thermal tracer test which is conducted by Bätzel (2017), where two possible hydraulic connections at depth of $18 \mathrm{~m}$ and $27 \mathrm{~m}$ are predicted (Figure 4.18). Furthermore, the reconstruction in Figure 4.17 even implies a diagonal hydraulic connection between O5 and M2. This connection is supported by the high $D f$ of test O5M2. 
(a) Inversion result

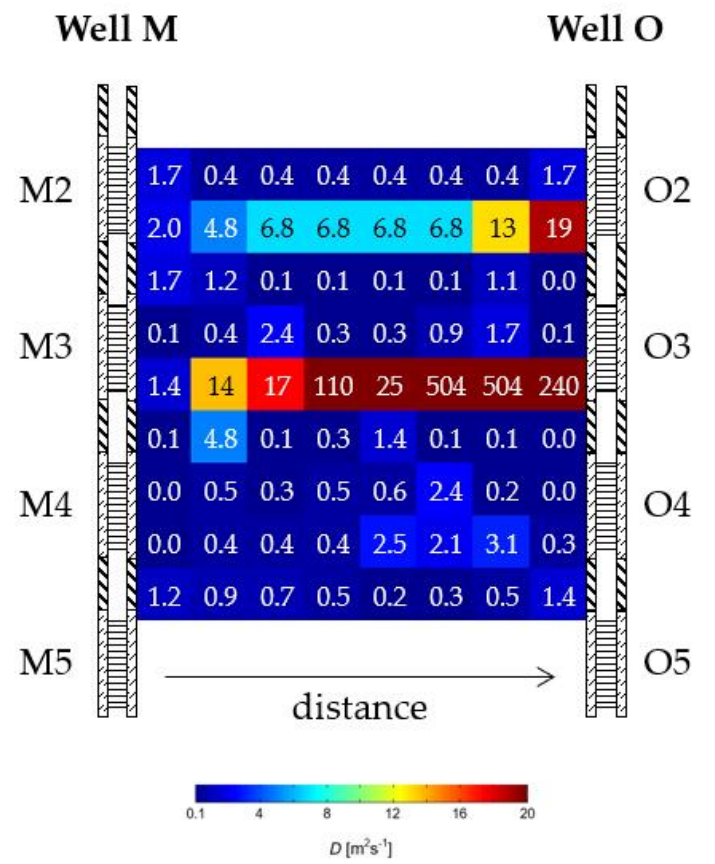

(b) Thermal tracer test result

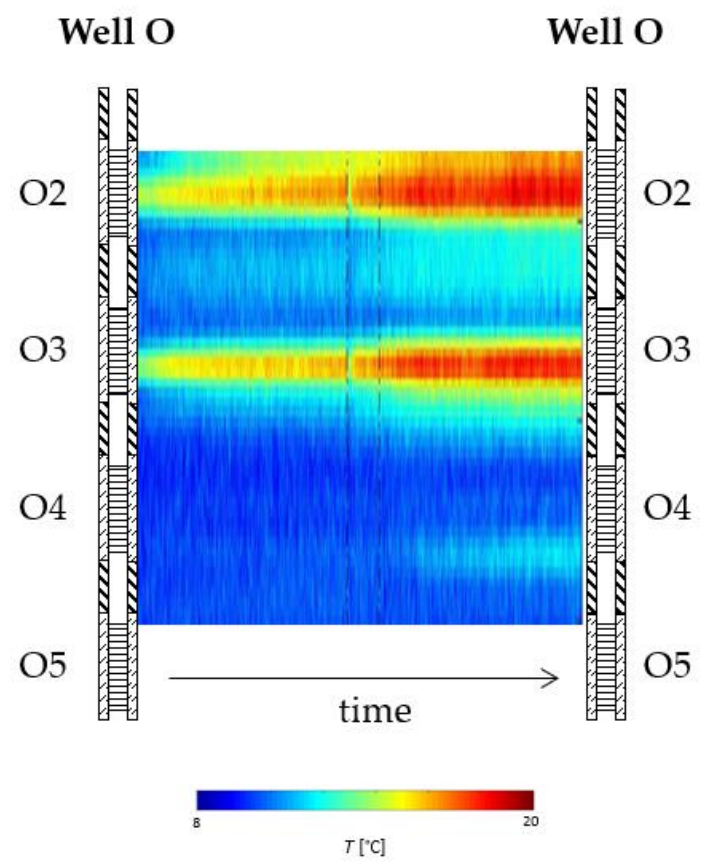

Figure 4.18. The comparison between (a) the SIRT-Cimmino inversion result under resolution $9 \times 8$ and (b) thermal tracer test result by Bätzel (2017). Note that, the inversion result shows the spatial diffusivity distribution while the thermal tracer test result shows the temperature development in well $\mathrm{O}$.

The $K f / K m$ and $D f$ values in Table 4.3 indicate a higher fracture density in the upper half of the investigation area. The SIRT-Common results confirm this density distribution in spite of the lack of information between O5 and M5.

In the end, the arithmetic average diffusivities are calculated (Table 4.5) and they have the same order of magnitude with the diffusivity obtained from the integral test $\left(7.5 \times 10^{1} \mathrm{~m}^{2} \mathrm{~s}^{-1}\right)$. Surprisingly, compared to SIRT-Cimmino result, all four SIRT results are closer to the estimation by using integral test. The reason is the setting of the max constraint. The average diffusivity will increase very fast when the SIRT inversion is performed under identical conditions but with a higher upper limit or without max constraint. Conversely, the min and max constraints do not affect the SIRT-Cimmino inversion result. 
From the abovementioned evaluation, we reach three following conclusions. (a) The high- $D$ zones in the reconstruction can be verified by type curve matching analysis. (b) The inversion using SIRT-Cimmino under high resolutions can exhibit secondly hydraulic connections. (c) The values in the distribution using SIRT-Cimmino are more reliable and accurate.

Table 4.5. The arithmetic average diffusivities $\left(D\left[\mathrm{~m}^{2} \mathrm{~s}^{-1}\right]\right)$ of the results by using SIRT (at 8th, 10th, 15th and 50th step) and SIRT-Cimmino.

\begin{tabular}{cccccc}
\hline \multicolumn{5}{c}{ SIRT } & SIRT-Cimmino \\
\hline & step 8 & step 10 & step 15 & step 50 & - \\
\hline Average $D$ & 39.0 & 65.7 & 78.3 & 112.0 & 10.7 \\
\hline
\end{tabular}

\subsection{Discussion}

Comparison with the type curve method and previous research (mainly thermal tracer test and geological survey) not only verifies the performance of algorithms, but also shows the advantage of the inversion on spatial distribution characterization. The validation of geological survey and thermal tracer test is limited in the vicinity of test well. As shown in Figure 4.18 (b), the temperature development yields responses at two depths in well $\mathrm{O}$, but the place of the origins in well $\mathrm{M}$ are not determined. That is to say, the thermal transport path can not be discovered through the thermal tracer test.

The inversion using field data should take more uncertainties into account, compared with the inversion using synthetic data. For example, noise, pumping rate, wellbore storage, skin effect, length of the screen and the interference of other wells.

(a) The travel time is based on the differentiation, which is strongly sensitive to the noise. Deviation is inevitable in spite of the application of denoising methods. The travel time derived from the recovery phase is more reliable due to 
the lack of noise caused by pump vibration and electromagnetic induction. However, the feasibility has not been theoretically validated and the difference to the travel time derived from the pumping phase has not been discussed.

(b) The flowmeter measurement shows the pumping rate can not achieve the present rate immediately. This problem affects the drawdown in the early phase, and further influences the estimation of the fracture specific storage and the travel time. In our case, the growth of the rate takes about 5 seconds, and we assume the rate increases in a linear manner.

(c) At the beginning of a pumping test, water is pumped initially from the wellbore storage, then from the gravel pack (skin), and lastly from the aquifer. The existence of wellbore storage and skin effect leads to a misestimation on the travel time. Three means can be considered to reduce the influence. (1) Fit the drawdown with solutions including wellbore storage and skin effect and derive the travel time directly from the analytical solutions. This mean depends strongly on the suitability of the solution and the matching performance. (2) Embed the gravel pack into the mesh and consider it as a special cell with unknown diffusivity. This adaption increases the computational cost and requires the inversion software featuring customizable mesh. (3) Apply the empirical conductivity and specific storage of gravel to quantify diffusivity and estimate the travel time in the gravel pack. This mean is used in our case due to its simplicity. On the basis of Bear (1972), Domenico and Mifflin (1965) and Judge (2013), $K: 1 \times 10^{-2}-1 \mathrm{~m} \cdot \mathrm{s}^{-1}, S_{s}: 5 \times 10^{-5}-1 \times$ $10^{-4} \mathrm{~m}^{-1}$, the diffusivity thus ranges from $1 \times 10^{2}-2 \times 10^{4} \mathrm{~m}^{2} \mathrm{~s}^{-1}$ and the travel time through $4.5 \mathrm{~cm}$ thick gravel pack is $2 \times 10^{-4}-3 \times 10^{-2} \mathrm{~s}$, which can be neglected compared with the observed travel time.

(d) Water in the well is assumed incompressible, the signal travels in the screen section with an infinite speed and the travel time is thus neglected. This assumption implies that any depth of the pumping screen can be used as the 
source location, and any depth of the observation screen can be used as the receiver location. In the test site, the screen is $5 \mathrm{~m}$ long and the horizontal distance between two wells is just $1.9 \mathrm{~m}$. This geometry enlarges the likelihood of deviation occurrence.

(e) Both algorithms are developed for 2D reconstruction by using the data from 3D aquifer. Some problems could be caused by this dimensional gap. For instance, the inference of other wells. We assume O2 and M4 are strongly connected with S2 and S4, respectively. The trajectory of the signal in test O2M4 could be O2 - S2 - S4-M4, when O2 and M4 are poorly hydraulic connected.

(f) The tomographic setting in field test is different to the previous three numerical tests. Because of the equipment limitation and the geometrical structure of the screens, the angles of source and receiver are smaller than those in the design. This modification eliminates the abnormal shape of the layers. 


\section{Conclusions and Outlook}

As the main objective of this dissertation, an inversion algorithm, SIRTCimmino, is developed to solve the shortcomings of SIRT. SIRT-Cimmino adopts the Cimmino iteration strategy with adjusted incremental corrections and an iteration-dependent relaxation parameter. The algorithm is verified by three numerical experiments and applied in a field test site in Göttingen, Germany. The SIRT-Cimmino shows its advantage to traditional inversion algorithm (i.e., SIRT), and a great potential for a more accurate and reliable characterization of diffusivity spatial distribution. The development of the algorithm, the analysis of the iteration step sensitivity, and the application of the tomographical idea shed lights on the understanding bridging mathematical science and practical problems.

\subsection{Conclusion}

The feasibility of SIRT-Cimmino and the residual based result selection rule are verified by three numerical models with different diffusivity distributions, i.e., inclined high- $D$ layer, Y-shaped high- $D$ zone, and aquifer analogue outcrop. In total, 44 pumping tests are simulated, and 520 travel times are obtained. The inversion results are compared to the true distribution, and the following conclusions are drawn.

- SIRT-Cimmino rebuilds the main feature (i.e., the high-D continuum) with better continuity and more precise values than SIRT.

- SIRT-Cimmino resolves the difficulty in determining the optimal number of iteration steps (NIS). The NIS, determined from the result selection rule in SIRT-Cimmino, provides a good agreement between structural similarity and numerical accuracy. Meanwhile in SIRT, the NIS with a satisfactory structure is not consistent with the NIS with an acceptable 
accuracy. This advantage has a great impact on the field study due to the lack of reference distribution.

- The investigation of SIRT-Cimmino under high resolution indicates that (a) the reconstructions are generally stable and reliable; (b) SIRT-Cimmino faces difficulties on the reconstruction of horizontal features, and deviations on the shape might occur.

Differs from the numerical studies, the field study is the first time of using travel time based hydraulic tomography in a fractured porous aquifer. 13 crosswell multi-level short term pumping tests are performed, and 13 travel times are obtained. The field study demonstrates that the distribution obtained by SIRTCimmino has a higher agreement with the result of the type curve analysis and previous thermal tracer test. This demonstration is concluded from two following observations.

- SIRT-Cimmino exhibits two high-D continua, which were found in the previous thermal tracer test. SIRT only rebuilds one continuum, i.e., the continuum at the lower part of the third screens.

- The values by using SIRT-Cimmino range in the proper interval, which is estimated by the type curve analysis of the cross-well multi-level pumping tests. SIRT overestimates diffusivities in some cells.

In overall, the models and field studies reveal that SIRT-Cimmino can offer great benefits over the traditional SIRT.

\subsection{Outlook}

The thoroughly examined the SIRT-Cimmino algorithm allows us to further understand and improve the travel time based hydraulic tomography. In particular, three concerns from mathematical, software and field perspectives can be addressed as follows. 
First, the travel time based hydraulic tomography is a nonlinear and ill-posed inverse problem. Three questions remain unanswered: (a) does a solution exist? (b) is the solution unique? (c) how do the initial and boundary conditions affect the solution?

(a) We assume that the measurement is errorless, the mesh is fine enough, the ray-tracing technique simulates the trajectory precisely, thus the existence of the solution only depends on the line integral bridging the diffusivity and travel time. Note that the derivation of the line integral is based on the smooth varying hydraulic parameter field. The feasibility of travel time based hydraulic tomography in a highly heterogenous test site is not promised. (b) SIRT-Cimmino and SIRT provide different approaches with small residuals. It implies the nonuniqueness of the solutions. Moreover, the relationship between non-uniqueness and grid resolution becomes complex due to nonlinearity. The oscillating behaviour in the residual need to be explained theoretically. (c) In general, the inversion is beneficially influenced by the appropriate initialization and constraint setting, which can be established through hydraulic test, geological survey, and previous research.

Second, ray-bending technique and 3D inversion could improve the inversion performance. The ray-bending technique provides curve segment and fines the trajectory simulation. The use of multiple raying techniques not only increase the accuracy, but also accelerates the iteration. This work is limited to the 2D inversion for an aquifer profile, the estimated trajectory is therefore the projection of trajectory in the 3D aquifer on the 2D profile. This dimensional gap might lead to a mischaracterization.

Lastly, the field application of an algorithm contains a lot of challenges, which stem from the difference between theoretical and field works. Noise, well construction, interference of other wells, and geometry of the study profile are 
critical points. They restrict the utilization of some methods, which can improve the result quality in the numerical experiment. For instance, the early time diagnostic. In this dissertation, kinds of countermeasures are implemented to overcome the difficulties or reduce the influence, but some of them might not suit other test sites due to the different field conditions. 


\section{References}

Agarwal, R. G. (1970) An investigation of wellbore storage and skin effects in unsteady liquid flow:1. analytical treatment. Society of Petroleum Engineers Journal, 10, 279289.

Barenblatt, G. I., I. P. Zheltov \& I. N. Kochina (1960) Basic concepts in the theory of seepage of homogeneous liquids in fissured rocks [strata]. Journal of Applied Mathematics and Mechanics, 24, 1286-1303.

Barker, J. A. (1988) A generalized radial flow model for hydraulic tests in fractured rock. Water Resources Research, 24, 1796-1804.

Bätzel, K. 2017. Hydrogeological characterization of a fractured aquifer based on modelling and heat tracer experiment. In Faculity of Geoscience and Geography. University of Goettingen.

Bauer, J. F., M. Krumbholz, S. Meier \& D. C. Tanner (2017) Predictability of properties of a fractured geothermal reservoir: the opportunities and limitations of an outcrop analogue study. Geothermal Energy, 5-24.

Bayer, P. 1999. Aquifer-Analog-Studie in grobklastischen braided river Ablagerungen: Sedimentäre/hydrogeologische Wandkartierung und Kalibrierung von Georadarmessung. University of Tübingen.

Bayer, P. \& M. Finkel (2004) Evolutionary algorithms for the optimization of advective control of contaminated aquifer zones. Water Resources Research, 40.

Bear, J. 1972. Dynamics of Fluids in Porous Media. New York: Dover publications.

Bear, J., C.-F. Tsang \& G. D. Marsily. 1993. Dynamics of Fluids in Fractured Rock. Elsevier Science.

Beauheim, R. L., R. M. Roberts \& J. D. Avis (2004) Well testing in fractured media: flow dimensions and diagnostic plots. Journal of Hydraulic Research, 42, 69-76.

Berg, S. J. \& W. A. Illman (2011) Three-dimensional transient hydraulic tomography in a highly heterogeneous glaciofluvial aquifer-aquitard system. Water Resources Research, 47.

--- (2012) Improved predictions of saturated and unsaturated zone drawdowns in a heterogeneous unconfined aquifer via transient hydraulic tomography: Laboratory sandbox experiments. Journal of Hydrology, 470-471, 172-183.

Berg, S. J. \& W. A. Illman (2015) Comparison of hydraulic tomography with traditional methods at a highly heterogeneous site. Ground Water, 53, 71-89.

Born, M. \& E. Wolf. 1999. Principle of Optics. Cambridge University Press, Cambridge, UK.

Boulton, N. S. \& T. D. Streltsova (1978) Unsteady Flow to a Pumped Well in a Fissured Aquifer With a Free Surface Level Maintained Constant. Water Resources Research, $14,527-532$.

Bourdet, D., J. A. Ayoub \& Y. M. Pirard (1989) Use of pressure derivative in well-test interpretation. SPE Formation Evaluation, 4, 293-302.

Bourdet, D., T. M. Whittle, A. A. Douglas \& Y. M. Pirard (1983) A new set of type curves simplifies well test analysis. World Oil, 196, 95-106. 
Braester, C. 1999. Radioactive Waste Repositories in Fractured Rocks Formations: Hydrodynamic Aspects. In Energy and the Environment, 229-238. Dordrecht: Springer.

Brauchler, R., G. Böhm, C. Leven, P. Dietrich \& M. Sauter (2013a) A laboratory study of tracer tomography. Hydrogeology Journal, 21, 1265-1274.

Brauchler, R., J. Doetsch, P. Dietrich \& M. Sauter (2012) Derivation of site-specific relationships between hydraulic parameters and p-wave velocities based on hydraulic and seismic tomography. Water Resources Research, 48.

Brauchler, R., R. Hu, P. Dietrich \& M. Sauter (2011) A field assessment of high-resolution aquifer characterization based on hydraulic travel time and hydraulic attenuation tomography. Water Resources Research, 47.

Brauchler, R., R. Hu, L. Hu, S. Jiménez, P. Bayer, P. Dietrich \& T. Ptak (2013b) Rapid field application of hydraulic tomography for resolving aquifer heterogeneity in unconsolidated sediments. Water Resources Research, 49, 2013-2024.

Brauchler, R., R. Hu, T. Vogt, D. Al-Halbouni, T. Heinrichs, T. Ptak \& M. Sauter (2010) Cross-well slug interference tests: An effective characterization method for resolving aquifer heterogeneity. Journal of Hydrology, 384, 33-45.

Brauchler, R., R. Liedl \& P. Dietrich (2003) A travel time based hydraulic tomographic approach. Water Resources Research, 39.

Brokešová, J. 2006. Asymptotic ray method in seismology: A tutorial. Prague, Czech Republic: Matfyzpress.

Bruneau, M. 2006. Fundamentals of Acoustics. ISTE, London, UK.

Butler, J. J. J. \& X. Zhan (2004) Hydraulic tests in highly permeable aquifers. Water Resources Research, 40.

Calayir, Y. \& M. Karaton (2005) Seismic fracture analysis of concrete gravity dams including dam-reservoir interaction. Computers \& Structures, 83, 1595-1606.

Cardiff, M., T. Bakhos, P. K. Kitanidis \& W. Barrash (2013) Aquifer heterogeneity characterization with oscillatory pumping: Sensitivity analysis and imaging potential. Water Resources Research, 49, 5395-5410.

Cardiff, M. \& W. Barrash (2011) 3-D transient hydraulic tomography in unconfined aquifers with fast drainage response. Water Resources Research, 47.

Carlson, M. R. (1999) Reservoir Characterization Of Fractured Reservoirs In Western Canada. Journal of Canadian Petroleum Technology, 38.

Chai, T. \& R. R. Draxler (2014) Root mean square error (RMSE) or mean absolute error (MAE)? - Arguments against avoiding RMSE in the literature. Geoscientific Model Development, 7, 1247-1250.

Chapman, S. J., J. M. H. Lawry \& J. R. Ockendono (1999) Ray theory for high-Pécletnumber convection-diffusion. SIAM Journal on Applied Mathematics, 60, 121-135.

Cimmino, G. (1938) Calcolo approssimato per le soluzioni dei sistemi di equazioni lineari. La Ricerca Scientifica XVI, 9, 326-333.

Cinco-Ley, H. \& F. Samaniego-V. (1981) Transient Pressure Analysis for Fractured Wells. Journal of Petroleum Technology, 33, 1749-1766. 
Clauser, C. (1992) Permeability of crystalline rocks. Eos, Transactions, American Geophysical Union, 73, 233-238.

Cook, P. G. 2003. A Guide to Regional Groundwater Flow in Fractured Rock Aquifers. Australia: CSIRO Land and Water.

Cooper, H. H. \& C. E. Jacob (1946) A generalized graphical method for evaluating formation constants and summarizing well field history. Transactions of the American Geophysical Union, 27, 526-534.

Dietrich, P., R. Helmig, M. Sauter, H. Hötzl, J. Köngeter \& G. Teutsch. 2004. Flow and Transport in Fractured Porous Media. Springer.

Dijkstra, E. W. (1959) A Note on Two Prblems in Connexion with Graphys. Numerische Mathematik, 1, 269-271.

Dingle, R. B. 1973. Asymptotic Expansions: Their Derivation and Interpretation. Academic Press, London, UK.

Domenico, P. A. \& M. D. Mifflin (1965) Water from Low-Permeability Sediments and Land Subsidence. Water Resources Research, 1, 563-576.

Dougherty, D. E. \& D. K. Babu (1984) Flow to a Partially Penetrating Well in a DoublePorosity Reservoir. Water Resources Research, 20, 1116-1122.

AQTESOLV for Windwos Version 4.5 User's Guide. 4.5, HydroSOLVE Inc., Reston, VA, USA.

Eggermont, P. P. B., G. T. Herman \& A. Lent (1981) Iterative algorithm for large partitioned linear systems, with applications to image reconstruction. Linear Algebra and its Applications, 40, 37-67.

Elble, J. M., N. V. Sahinidis \& P. Vouzis (2010) GPU computing with Kaczmarz's and other iterative algorithms for linear systems. Parallel Comput, 36, 215-231.

Elfving, T., T. Nikazad \& P. C. Hansen (2010) Semi-convergence and relaxation parameters for a class of SIRT algorithms. Electronic Transactions on Numerical Analysis, 37, 321-336.

Engelder, T. 1987. Joints and Shear Fractures in Rock. In Fracture Mechanics of Rock, ed. B. K. Atkinson. Academic Press.

Eskicioglu, A. M. \& P. S. Fisher (1995) Image quality measures and their performance. IEEE Transactions on Communications, 43, 2959-2965.

Evans, D. D., T. J. Nicholson \& T. C. Rasmussen. 2001. Flow and Transport through Unsaturated Fractured Rock. Washington D.C.: American Geophysical Union.

Evans, I. \& C. D. Pomeroy. 1966. The strength, fracture and workability of coal: a monograph on basic work on coal winning carried out by the Mining Research Establishment, National Coal Board. Pergamon Press.

Fatemi, E., B. Engquist \& S. Osher (1995) Numerical solution of the high frequency asymptotic expansion for the scalar wave equation. Journal of Computational Physics, 120, 145-155.

Faybishenko, B., P. A. Witherspoon \& S. M. Benson. 2000. Dynamics of Fluids in Fractured Rock. Washington D. C.: American Geophysical Union.

Fienen, M. N., T. Clemo \& P. K. Kitanidis (2008) An interactive bayesian geostatistical inverse protocol for hydraulic tomography. Water Resources Research, 44. 
Gilbert, P. (1972) Iterative methods for the three-dimensional reconstruction of an object from projections. Journal of Theoretical Biology, 36, 105-117.

Golf-Racht, T. D. v. 1982. Fundamentals of Fractured Reservoir Engineering.

Gordon, R., R. Bender \& G. T. Herman (1970) Algebraic reconstruction techniques (ART) for three-dimensional electron microscopy and X-Ray photography. Journal of Theoretical Biology, 29, 471-481.

Gringarten, A. C. (1987) Type-Curve Analysis: What It Can and Cannot Do. Journal of Petroleum Technology, 39.

Gringarten, A. C., D. P. Bourdet, P. A. Landel \& V. J. Kniazeff. 1979. A comparison between different skin and wellbore storage type-curves for early-time transient analysis. In 54th Annual Fall Technique Conference and Exhibition of the Society of Petroleum Engineers of AIME. Las Vegas, Nevada.

Gringarten, A. C. \& H. J. Ramey (1974) Unsteady state pressure distributions created by a well with a single horizontal fracture, partial penetration or restricted entry. SPE Journal, 413-426.

Gringarten, A. C. \& P. A. Witherspoon. 1972. A method of analyzing pump test data from fractured aquifers. In Int. Soc. Rock Mechanics and Int. Assoc. Eng. Geol., 1-9. Stuttgart: Proc. Symp. Rock Mechanics.

Gudmundsson, A. 2011. Rock Fractures in Geological Processes. Cambridge University Press.

Hammond, P. A. (2017) Reliable yields of public water-supply wells in the fracturedrock aquifers of central Maryland, USA. Hydrogeology Journal, 26, 333-349.

Hansan, P. C. \& M. Saxild-Hansan (2012) AIR Tools - A MATLAB package of algebraic iterative reconstruction methods. Journal of Computational and Applied Mathematics, 236, 2167-2178.

Hantush, M. S. (1961a) Aquifer tests on partially penetrating wells. Journal of the Hydraulics Division, 87, 171-194.

--- (1961b) Drawdown around a partially penetrating well. Journal of the Hydraulics Division, 87, 83-98.

Hantush, M. S. \& C. E. Jacob (1955a) Non-steady Green's functions for an infinite strip of leaky aquifer. Trans. Am. Geophys. Union, 36, 101-112.

Hantush, M. S. \& C. E. Jacob (1955b) Non-steady radial flow in an infinite leaky aquifer. Am. Geophys. Union Trans., 36, 95-100.

Hao, Y., T. C. Yeh, B. Han, J. Xiang, F. Zhu \& C. Ni (2008) Imaging fracture connectivity hydraulic tomography. Hydrogeology E Engineering Geology, 6, 6-11.

He, Z., A. Datta-Gupta \& D. W. Vasco (2006) Rapid inverse modeling of pressure interference tests using trajectory-based traveltime and amplitude sensitivities. Water Resources Research, 42.

Heath, R. C. 1983. Basic ground-water hydrology. Reston, VA.

Heinz, J., S. Kleineidam, G. Teutsch \& T. Aigner (2003) Heterogeneity patterns of Quaternary glaciofluvial gravel bodies (WE-Germany): application to hydrogeology. Sedimentary Geology, 158, 1-23. 
Hochstetler, D. L., W. Barrash, C. Leven, M. Cardiff, F. Chidichimo \& P. K. Kitanidis (2016) Hydraulic tomography: continuity and discontinuity of high-K and low-K zones. Ground Water, 54, 171-85.

Horne, R. N. (1994) Advances in computer-aided well-test interpretation. Journal of Petroleum Technology, 46, 599-606.

Hu, L., P. Bayer, P. Alt-Epping, A. Tatomir, M. Sauter \& R. Brauchler (2015) Time-lapse pressure tomography for characterizing $\mathrm{CO} 2$ plume evolution in a deep saline aquifer. International Journal of Greenhouse Gas Control, 39, 91-106.

Hu, R. 2011. Hydraulic Tomography: A New Approach Coupling hydraulic Travel Time , Attenuation and Steady Shape Inversions for High-Spatial Resolution Aquifer Characterization. In Applied Geology. University of Goettingen.

Hu, R., R. Brauchler, M. Herold \& P. Bayer (2011) Hydraulic tomography analog outcrop study: Combining travel time and steady shape inversion. Journal of Hydrology, 409, 350-362.

Hu, R., W. Zhao \& R. Brauchler. 2010. An aquifer analogue study of high resolution aquifer characterization based on hydraulic tomography. In Geoshanghai 2010 International Conference. Shanghai: Geoenvironmental Engineering and Geotechnis.

Hughson, D. L. \& T.-C. J. Yeh (2000) An inverse model for three-dimensional flow in variably saturated porous media. Water Resources Research, 36, 829-839.

Illman, W. A. (2014) Hydraulic tomography offers improved imaging of heterogeneity in fractured rocks. Groundwater, 52, 659-684.

--- (2015) Lessons learned from hydraulic and pneumatic tomography in fractured rocks. Procedia Environmental Sciences, 25, 127-134.

Illman, W. A., A. J. Craig \& X. Liu (2008) Practical issues in imaging hydraulic conductivity through hydraulic tomography. Ground Water, 46, 120-32.

Illman, W. A., X. Liu \& A. Craig (2007) Steady-state hydraulic tomography in a laboratory aquifer with deterministic heterogeneity: Multi-method and multiscale validation of hydraulic conductivity tomograms. Journal of Hydrology, 341, 222-234.

Illman, W. A., X. Liu, S. Takeuchi, T.-C. J. Yeh, K. Ando \& H. Saegusa (2009) Hydraulic tomography in fractured granite: Mizunami underground research site, Japan. Water Resources Research, 45.

Ismail, S., S. Mansor, A. Rodsi \& B. K. Bujang (2011) Geotechnical modeling of fractures and cavities that are associated with geotechnical engineering problems in Kuala Lumpur limestone, Malaysia. Environmental Earth Sciences, 62, 61-68.

Jackson, M. J. \& D. R. Tweeton. 1996. 3DTOM: Three-dimensional geophysical tomography.

Judge, A. 2013. Measurement of the hydraulic conductivity of gravels using a laboratory permeameter and silty sands using field testing with observation wells. University of Massachusetts Amherst.

Kaczmarz, S. (1937) Angenäherte Auflösung von Systemen linearer Gleichungen. Bulletin International de l'Académie Polonaise des Sciences et des Lettres, 35, 355-357. 
Kitanidis, P. K. (1995) Quasi-linear geostatistical theory for inversing. Water Resources Research, 31, 2411-2419.

Kostic, B., A. Becht \& T. Aigner (2005) 3-D sedimentary architecture of a Quaternary gravel delta (SW-Germany): Implications for hydrostratigraphy. Sedimentary Geology, 181, 143-171.

Kruseman, G. P. \& N. A. d. Ridder. 1994. Analysis and Evaluation of Pumping Test Data. Wageningen, the Netherlands: Intern. Inst. for Land Reclamation and Improvement.

Kulkarni, K. N., A. Datta-Gupta \& D. W. Vasco. 2001. A streamline approach for integrating transient pressure data into high-resolution reservoir models. In 2000 SPE European Petroleum Conference. SPE Journal.

Lee, J. \& P. K. Kitanidis (2014) Large-scale hydraulic tomography and joint inversion of head and tracer data using the Principal Component Geostatistical Approach (PCGA). Water Resources Research, 50, 5410-5427.

Liedl, R. \& T. Ptak (2003) Modelling of diffusion-limited retardation of contaminants in hydraulically and lithologically nonuniform media. Journal of Contaminant Hydrology, 66, 239-259.

Liu, S., T. C. J. Yeh \& R. Gardiner (2002) Effectiveness of hydraulic tomography: Sandbox experiments. Water Resources Research, 38, 5-1-5-9.

Liu, X., W. A. Illman, A. J. Craig, J. Zhu \& T. C. J. Yeh (2007) Laboratory sandbox validation of transient hydraulic tomography. Water Resources Research, 43.

Lo, T.-w. \& P. L. Inderwiesen. 1994. Fundamentals of seismic tomography. In Geophysical Monograph Series. Society of Exploration Geophysicists.

Long, J. C. S., J. S. Remer, C. R. Wilson \& P. A. Witherspoon (1982) Porous Media Equivalents for Networks of Discontinuous Fractures. Water Resources Research, 18, 645-658.

Maji, R. \& E. A. Sudicky (2008) Influence of mass transfer characteristics for DNAPL source depletion and contaminant flux in a highly characterized glaciofluvial aquifer. Journal of Contaminant Hydrology 102, 105-119.

Maliva, R. G. 2016. Aquifer Characterization and Properties. In Aquifer Characterization Techniques, 1-24.

Matthäi, S. K. \& M. Belayneh (2004) Fluid flow partitioning between fractures and a permeable rock matrix. GEOPHYSICAL RESEARCH LETTERS, 31.

McElwee, C. D. (2001) Application of a Nonlinear Slug Test Model. Ground Water, 39, 737-744.

Moench, A. F. (1984) Double-porosity models for a fissured groundwater reservoir with fracture skin. Water Resources Research, 20, 831-846.

---. 1988. The response of partially penetrating wells to pumpage from double-porosity aquifers. In Symposium Proceedings of International Conference on Fluid Flow in Fractured Rocks. Atlanta, Georgia.

--- (1997) Flow to a well of finite diameter in a homogeneous, anisotropic water table aquifer. Water Resources Research, 33, 1397-1407.

Moser, T. J. (1991) Shortest path calculation of seismic rays. Geophysics, 56, 59-67. 
Müller, C., S. Siegesmund \& P. Blum (2010) Evaluation of the representative elementary volume (REV) of a fractured geothermal sandstone reservoir. Environmental Earth Sciences, 61, 1713-1724.

Nakanishi, I. \& K. Yamaguchi (1986) A numerical experiment on nonlinear image reconstruction from first-arrival times for two-dimensional island arc structure. Journal of Physics of the Earth, 34, 195-201.

Nelson, R. A. 2001. Geologic Analysis of Naturally Fractured Reservoirs. Elsevier.

Neuman, S. P. (1974) Effect of partial penetration on flow in unconfined aquifers considering delayed gravity response. Water Resources Research, 10, 303-312.

--- (1979) Perspective on 'Delayed Yield'. Water Resources Research, 15, 899-908.

Neuman, S. P. \& P. A. Witherspoon (1969) Theory of flow in a confined two aquifer system. Water Resources Research, 5, 803-816.

Nolet, G. 2008. A breviary of seismic tomography Cambridge University Press.

NRC, N. R. C. 1996. Rock Fractures and Fluid Flow: Contemporary Understanding and Applications. National Academy Press.

Oberdorfer, P., E. Holzbecher, R. Hu, T. Ptak \& M. Sauter. 2013. A five spot cluster for hydraulic and thermal tomography. In Thirty-Eighth Workshop on Geothermal Reservoir Engineering. Stanford University, Stanford, California.

Olver, F. W. J. 1997. Asymptotic and Special Functions. New York, USA: Academic Press.

Papadopulos, I. S. \& H. H. Cooper (1967) Drawdown in a well of large diameter. Water Resources Research, 3, 241-244.

Perkins, T. K. \& R. G. Keck. 1994. Waste disposal in hydraulically fractured earth formations. United States.

Piecha, B. 2008. Untersuchungen zur Wärmeleitfähigkeit des Göttinger Untergrunds im Rahmen der Nutzung oberflächennaher Erdwärme. In Faculty of Geoscience and Geography. University of Goettingen.

Pusch, R. 1994. Waste Disposal in Rock. Elsevier.

Qiao, L., L. Li \& Z. Chen (2013) Accelerated SIRT algorithm based on multigrid. CT Theory and Application, 22, 25-31.

Qiu, P., R. Hu, L. Hu, Q. Liu, Y. Xing, H. Yang, J. Qi \& T. Ptak (2019) A numerical study on travel time based hydraulic tomography using the SIRT algorithm with cimmino iteration. Water, 11, 909.

Renard, P., D. Glenz \& M. Mejias (2008) Understanding diagnostic plots for well-test interpretation. Hydrogeology Journal, 17, 589-600.

Santos, L. T. D. (1987) A parallel subgradient projections method for the convex feasibility problem. Journal of Computational and Applied Mathematics, 18, 307-320.

Saouma, V. E., J. J. Broz, E. Brühwiler \& H. L. Boggs (1991) Effect of Aggregate and Specimen Size on Fracture Properties of Dam Concrete. Journal of Materials in Civil Engineering, 3.

Schöniger, A., W. Nowak \& H. J. Hendricks Franssen (2012) Parameter estimation by ensemble Kalman filters with transformed data: Approach and application to hydraulic tomography. Water Resources Research, 48. 
Schuster, V. 2015. Untersuchungen zur hydraulischen Durchlässigkeit und kluftbedingter Durchlässigkeitsanisotropie von Bohrkernmaterial aus einem Geothermietestfeld. In Faculty of Geoscience and Geography. University of Goettingen.

Scudder, H. J. (1978) Introduction to computer aided tomography. Proceedings of the IEEE, 66.

Shih, D. C.-F. (2018) Hydraulic diffusivity in a coastal aquifer: spectral analysis of groundwater level in responses to marine system. Stochastic Environmental Research and Risk Assessment, 32, 311-320.

Shrestha, J. G. 2013. Design of thermal tomography tests on the basis of a heterogeneous flow model. In Faculty of Geoscience and Geography. University of Goettingen.

Spane, F. A., Jr. \& S. K. Wurstner (1993) DERIV: A computer program for calculating pressure derivatives for use in hydraulic test analysis. Ground Water, 31, 814-822.

Streltsova, T. D. (1977) Hydrodynamics of groundwater flow in a fractured formation. Water Resources Research, 12, 405-414.

Streltsova, T. D. 1988. Well testing in heterogeneous formations. United States: Wiley.

Sun, R., T.-C. J. Yeh, D. Mao, M. Jin, W. Lu \& Y. Hao (2013) A temporal sampling strategy for hydraulic tomography analysis. Water Resources Research, 49, 38813896.

Sun, X., Y. Xu \& L. Lin (2015) The diagnostic plot analysis of artesian aquifers with case studies in Table Mountain Group of South Africa. Hydrogeology Journal, 23, 567579 .

Taherdangkoo, R. \& M. Abdideh (2016) Fracture density estimation from well logs data using regression analysis: validation based on image logs (Case study: South West Iran). Int. J. Petroleum Engineering, 2, 289-301.

Taherdangkoo, R., A. Tatomir, T. Anighoro \& M. Sauter (2019) Modeling fate and transport of hydraulic fracturing fluid in the presence of abandoned wells. Journal of Contaminant Hydrology, 221, 58-68.

Tan, E. Y. Z. 2015. Hydraulic tomography of a fractured aquifer based on a ray tracing method. In Faculty of Geoscience and Geography. University of Goettingen.

Tarantola, A. 2005. Inverse problem theory. Society of Industrial and Applied Mathematics.

Tartakovsky, G. D. \& S. P. Neuman (2007) Three-dimensional saturated-unsaturated flow with axial symmetry to a partially penetrating well in a compressible unconfined aquifer. Water Resources Research, 43.

Theis, C. V. (1935) The relation between the lowering of the piezometric surface and the rate and duration of discharge of a well using groundwater storage. Transactions of the American Geophysical Union, 16, 519-524.

Thomas, F. B. \& D. B. Bennion (2007) Fractured Gas-Reservoir Performance. Journal of Canadian Petroleum Technology, 46.

Tsang, C. F., I. Neretnieks \& Y. Tsang (2015) Hydrologic issues associated with nuclear waste repositories. Water Resources Research, 51, 6923-6972. 
Tsoflias, G. P., T. Halihan \& J. M. S. Jr. (2001) Monitoring pumping test response in a fractured aquifer using ground-penetrating radar. Water Resources Research, 37, 1221-1229.

Vallejo, L. E. \& R. Y. Liang. 1994. Fracture mechanics applied to geotechnical engineering. In American Society of Civil Engineers in conjunction with the ASCE National Convention. Atlanta, Georgia: American Society of Civil Engineers.

Vasco, D. W., H. Keers \& K. Karasaki (2000) Estimation of reservoir properties using transient pressure data: An asymptotic approach. Water Resources Research, 36, 3447-3465.

Virieux, J., C. Flores-Luna \& D. Gibert (1994) Asymptotic theory for diffusive electromagnetic imaging. Geophysical Journal International, 119, 857-868.

Wang, Z., A. C. Bovik, H. R. Sheikh \& E. P. Simoncelli (2004) Image quality assessment: From error visibility to structural similarity. IEEE Transactions on Image Processing, 13, 600-612.

Warren, J. E. \& P. J. Root (1963) The Behavior of Naturally Fractured Reservoirs. Society of Petroleum Engineers, 3.

Wei, X.-q., H.-b. Bai, H.-r. Rong, Y. Jiao \& B.-y. Zhang (2011) Research on Mining Fracture of Overburden in Close Distance Multi-seam. Procedia Earth and Planetary Science, 2, 20-27.

Wen, C. 2014. Aquifer characterization based on hydraulic tomography with steady shape inversion approach. In Faculity of Geoscience and Geography. University of Goettingen.

Werner, H. 2013. Strukturgeologische Charakterisierung eines Geothermietestfeldes auf der Basis bohrlochgeophysikalischer Messdaten und Bohrkerngefügen auf dem Göttinger Nordcampus. In Geowissenschaftlichen Zentrum der Universität Göttingen. University of Goettingen.

Wu, C.-M., T.-C. J. Yeh, J. Zhu, T. H. Lee, N.-S. Hsu, C.-H. Chen \& A. F. Sancho (2005) Traditional analysis of aquifer tests: Comparing apples to oranges? Water Resources Research, 41.

Xiang, J., T.-C. J. Yeh, C.-H. Lee, K.-C. Hsu \& J.-C. Wen (2009) A simultaneous successive linear estimator and a guide for hydraulic tomography analysis. Water Resources Research, 45.

Xiao, L. \& Y. Xu (2014) Diagnostic Analysis of Pumping Tests Using Derivative of dlgs/dlgt with Case Study. Groundwater, 52, 208-217.

Yang, H. 2019. The application of travel-time based hydraulic tomography for resolving the heterogeneity of a porous-fractured aquifer. In Applied Geology. University of Göttingen.

Yeh, T.-C. J., M. Jin \& S. Hanna (1996) An iterative stochastic inverse method: Conditional transmissivity and hydraulic head fields. Water Resources Research, 32, 85-92.

Yeh, T. C., D. Mao, Y. Zha, K. C. Hsu, C. H. Lee, J. C. Wen, W. Lu \& J. Yang (2014) Why hydraulic tomography works? Ground Water, 52, 168-72. 
Yeh, T. C. J. \& S. Liu (2000) Hydraulic tomography: Development of a new aquifer test method. Water Resources Research, 36, 2095-2105.

Zha, Y., T.-C. J. Yeh, W. A. Illman, H. Onoe, C. M. W. Mok, J.-C. Wen, S.-Y. Huang \& W. Wang (2017) Incorporating geologic information into hydraulic tomography: A general framework based on geostatistical approach. Water Resources Research, 53, 2850-2876.

Zha, Y., T. C. J. Yeh, W. A. Illman, W. Zeng, Y. Zhang, F. Sun \& L. Shi (2018) A reducedorder successive linear estimator for geostatistical inversion and its application in hydraulic tomography. Water Resources Research, 54, 1616-1632.

Zhang, W., J. Xu \& R. Jiang (2017) Production forecast of fractured shale gas reservoir considering multi-scale gas flow. Journal of Petroleum Exploration and Production Technology, 7, 1071-1083.

Zhao, H. (2004) A fast sweeping method for eikonal equations. Mathematics of Computation, 74, 603-627.

Zhao, Z., W. A. Illman, T.-C. J. Yeh, S. J. Berg \& D. Mao (2015) Validation of hydraulic tomography in an unconfined aquifer: A controlled sandbox study. Water Resources Research, 51, 4137-4155.

Zhu, J. \& T.-C. J. Yeh (2005) Characterization of aquifer heterogeneity using transient hydraulic tomography. Water Resources Research, 41. 


\section{Appendix}

\subsection{The transformation of diffusion equation by applying Fourier Transform}

This derivation is provided for the transformation of the diffusion equation by applying the Fourier transform. The diffusion equation for transient pressure response in a heterogeneous permeable medium is given with the boundary conditions,

$$
\begin{gathered}
\Delta P+\frac{\nabla K(x)}{K(x)} \cdot \nabla P-\frac{1}{D(x)} \frac{\partial P(x, t)}{\partial t}=0, \\
P( \pm \infty, t)=0,
\end{gathered}
$$

where $P(x, t)$ is the pressure, $K(x)$ is the permeability, and $D(x)$ is the diffusivity. For convenience, we use $P, K$ and $D$ for $P(x, t), K(x)$ and $D(x)$, respectively.

The Fourier transform of a function $f$ is defined as

$$
\mathcal{F}(f)=\int_{-\infty}^{+\infty} f e^{-i \omega t} d t .
$$

In our case, the Fourier transform of the pressure function $P(x, t)$ is given as

$$
\mathcal{F}(P)=\hat{P}=\int_{-\infty}^{+\infty} P(x, t) e^{-i \omega t} d t .
$$

Three properties need to be introduced first:

$$
\begin{gathered}
\mathcal{F}\left(\frac{\partial P}{\partial t}\right)=i \omega \mathcal{F}(p), \\
\mathcal{F}(\nabla P)=\nabla \mathcal{F}(P), \\
\mathcal{F}(\triangle P)=\triangle \mathcal{F}(P) .
\end{gathered}
$$

Proof of P1, 


$$
\begin{aligned}
& \mathcal{F}\left(\frac{\partial P}{\partial t}\right)=\int_{-\infty}^{+\infty} \frac{\partial P(x, t)}{\partial t} e^{-i \omega t} d t \\
= & {\left[p(x, t) e^{-i \omega t}\right]_{-\infty}^{+\infty}-\int_{-\infty}^{+\infty} P(x, t) \frac{\partial e^{-i \omega t}}{\partial t} d t } \\
= & 0-(-i \omega) \int_{-\infty}^{+\infty} P(x, t) e^{-i \omega t} d t \\
= & i \omega \mathcal{F}(p),
\end{aligned}
$$

where integration by parts and boundary conditions are used.

Proof of P2,

$$
\mathcal{F}\left(\frac{\partial P}{\partial x}\right)=\int_{-\infty}^{+\infty} \frac{\partial P}{\partial x} e^{-i \omega t} d t=\int_{-\infty}^{+\infty} \frac{\partial P e^{-i \omega t}}{\partial x} d t=\frac{\partial}{\partial x} \int_{-\infty}^{+\infty} P e^{-i \omega t} d t=\frac{\partial}{\partial x} \mathcal{F}(P)
$$

By substituting variables

$$
\mathcal{F}\left(\frac{\partial P}{\partial y}\right)=\frac{\partial}{\partial y} \mathcal{F}(P), \mathcal{F}\left(\frac{\partial P}{\partial z}\right)=\frac{\partial}{\partial z} \mathcal{F}(P)
$$

Then,

$$
\mathcal{F}(\nabla P)=\mathcal{F}\left(\frac{\partial P}{\partial x}, \frac{\partial P}{\partial y}, \frac{\partial P}{\partial z}\right)=\left(\frac{\partial \mathcal{F}(P)}{\partial x}, \frac{\partial \mathcal{F}(P)}{\partial y}, \frac{\partial \mathcal{F}(P)}{\partial z}\right)=\nabla \mathcal{F}(P)
$$

Proof of P3,

$$
\mathcal{F}\left(\frac{\partial^{2} P}{\partial x^{2}}\right)=\frac{\partial}{\partial x} \mathcal{F}\left(\frac{\partial P}{\partial x}\right)=\frac{\partial^{2}}{\partial x^{2}} \mathcal{F}(P)
$$

By substituting variables

$$
\mathcal{F}\left(\frac{\partial^{2} P}{\partial y^{2}}\right)=\frac{\partial^{2}}{\partial y^{2}} \mathcal{F}(P), \mathcal{F}\left(\frac{\partial^{2} P}{\partial z^{2}}\right)=\frac{\partial^{2}}{\partial z^{2}} \mathcal{F}(P)
$$

Then, 


$$
\mathcal{F}(\triangle P)=\mathcal{F}\left(\frac{\partial^{2} P}{\partial x^{2}}+\frac{\partial^{2} P}{\partial y^{2}}+\frac{\partial^{2} P}{\partial z^{2}}\right)=\frac{\partial^{2} \mathcal{F}(P)}{\partial x^{2}}+\frac{\partial^{2} \mathcal{F}(P)}{\partial y^{2}}+\frac{\partial^{2} \mathcal{F}(P)}{\partial z^{2}}=\triangle \mathcal{F}(P)
$$

Finally, we take the Fourier transform on both sides of the diffusion equation, i.e.,

$$
\begin{gathered}
\mathcal{F}\left(\triangle P+\frac{\nabla K}{K} \cdot \nabla P-\frac{1}{D} \frac{\partial P}{\partial t}\right)=0, \\
\mathcal{F}(\triangle P)+\mathcal{F}\left(\frac{\nabla K}{K} \cdot \nabla P\right)-\mathcal{F}\left(\frac{1}{D} \frac{\partial P}{\partial t}\right)=0, \\
\mathcal{F}(\triangle P)+\frac{\nabla K}{K} \cdot \mathcal{F}(\nabla P)-\frac{1}{D} \mathcal{F}\left(\frac{\partial P}{\partial t}\right)=0,
\end{gathered}
$$

where we acknowledge the linearity of the Fourier transform in moving the function $n, K$ out, since $n$ only depends on $x$. By using properties $1-3$, the transformation is derived

$$
\triangle \mathcal{F}(P)+\frac{\nabla K}{K} \cdot \nabla \mathcal{F}(P)-\frac{1}{D} i \omega \mathcal{F}(P)=0
$$

\subsection{Helmholtz equation degrades to the eikonal equation}

Helmholtz equation is given as

$$
\triangle u+n^{2}(x) \omega^{2} u=0
$$

where $u$ is the amplitude, $n(x)$ is the wave number, and $\omega$ is the angular frequency. We use asymptotic series expansion to approach the solution,

$$
u=e^{i \omega \emptyset(x)} \sum_{k=0}^{\infty} a_{k}(x)(i \omega)^{-k}
$$

We substitute the expansion into the Helmholtz equation and obtain

$$
\omega^{2}\left(n^{2}(x)-|\nabla \emptyset(x)|^{2}\right) u+i \omega\left(2 \nabla \emptyset(x) \cdot \nabla a_{0}+\triangle \emptyset(x) a_{0}\right) e^{i \omega \emptyset(x)}+O\left(\omega^{0}\right)=0
$$


Letting $\omega \rightarrow \infty$,

$$
|\nabla \emptyset(x)|=n(x) .
$$

The high frequency Helmholtz equation is then degraded to the eikonal equation.

\subsection{Software TomoGo}

TomoGo is a program for reconstructing the diffusivity distribution of aquifers. It was programmed in the $\mathrm{C} \#$ language and was first available in the year 2018 (Qiu et al. 2019). It functions on a normal computer with standard hardware and the Microsoft Windows operating system. Pengxiang Qiu (pengxiang.qiu@gmx.de) and Rui Hu are two main developers.

TomoGo can perform the steps in TTHT, including data pre-processing, travel time calculation, and inversion (diffusivity distribution reconstruction). Different types of travel times can be calculated (e.g., early travel time, late travel time). A variety of settings are provided in the core inversion part: investigation area, inversion algorithms, reconstruction resolution, individual and uniform initial distribution, prior information, value limit, number of iterations, and criterion types (Yang 2019).

Additionally, a visualization of the reconstructed diffusivity distribution is also provided by TomoGo. For each iteration, the distribution and the diffusivity value in each cell can be displayed in a user predefined grid with or without the different level interpolation effect. The signal trajectory between every source and receiver is drawn for better understanding of signal propagation. The deviation between the true trajectory and the estimated trajectory from the ray-tracing technique is the residual between the observed travel time and estimated travel 
time. This residual is measured in various metrics and is shown individually for each source and receiver pair. The residual of each iteration is summarized, and a trend is shown to help with user understanding of the convergence of the utilized algorithm.

\subsection{Root-Mean-Square Error}

Root-Mean-Square Error (RMSE) is the most frequently used measure of the deviation between values predicted or estimated by a model and the observed values. The RMSE is defined as

$$
R M S E=\sqrt{\frac{\sum_{i=1}^{n}\left(\hat{y}_{i}-y_{i}\right)^{2}}{n}},
$$

where $\hat{y}$ is an $n$-dimensional vector containing $n$ predicted or estimated values by a model and $y$ is the corresponding $n$-dimensional vector containing $n$ observed values.

RMSE is a non-negative number, with a value of 0 indicating a perfect predication or estimation. A lower RMSE is generally better than a higher one. However, since this measure is a scale dependent parameter (RMSE is a function of $n$ ), it is not used in cross-model comparisons where the value-dimension is different.

In this work, the predefined "truth" and the estimated diffusivity distributions are studied in two dimensions. RMSE estimates the difference between two distribution images on a "pixel-by-pixel" basis (Chai and Draxler 2014) and is defined by:

$$
R M S E=\sqrt{\frac{\sum_{i=1}^{n}\left(D_{i}^{e s t}-D_{i}\right)^{2}}{n}},
$$


where $D_{i}^{e s t}$ and $D_{i}$ represent the estimated and original diffusivity in the $i$ th cell, respectively.

RMSE is the simplest evaluation for image quality assessment, however, sometimes it differs from subjective measures (Eskicioglu and Fisher 1995). We therefore suggest that other numerical measures be used in combination with RMSE.

\subsection{Correlation Coefficient}

A correlation coefficient is a numerical measure that calculates the relationship between two variables in probability theory. Several types of correlation coefficient have been developed, but the Pearson correlation coefficient is the most commonly used and is applied in this study. The Pearson correlation coefficient $C$ is defined as the ratio of the covariance of two variables and the product of their standard deviation. The definition is described as follows

$$
C=\frac{\operatorname{cov}(X, Y)}{\sigma_{X} \sigma_{Y}},
$$

where $X$ and $Y$ are two random variables, cov is their covariance, and $\sigma$ is their standard deviation.

The value of $C$ always ranges between -1 and 1 . A value of 1 indicates a perfect positive relationship between the two variables, meaning that both variables move in the same direction. A value of -1 means a perfect negative relationship between the two variables, indicating that two variables move in opposite directions (e.g., a positive increase in one variable, while a decrease in the second variable). No relationship exists between the two variables if $C=0$.

In image quality assessment, the correlation coefficient is a simple and effective measure to quantify the structural similarity between two images. In our 
case, the diffusivity distribution can be considered a two-dimensional image, and the correlation coefficient therefore can also be applied in our study to measure the similarity between two distributions. Eq. (8.3.1) can be written as

$$
C=\frac{\sum_{i}^{n}\left(D_{i}^{\text {est }}-\overline{D^{e s t}}\right)\left(D_{i}-\bar{D}\right)}{\sqrt{\left(\sum_{i}^{n}\left(D_{i}^{\text {est }}-\overline{D^{e s t}}\right)^{2}\right)\left(\sum_{i}^{n}\left(D_{i}-\bar{D}\right)^{2}\right)}}
$$

where $D_{i}^{e s t}$ and $D_{i}$ represent the estimated and predefined ("true") diffusivities, respectively, in the $i$ th cell (Wang et al. 2004), and

$$
\overline{D^{e s t}}=\frac{1}{n} \sum_{i=1}^{n} D_{i}^{e s t}, \bar{D}=\frac{1}{n} \sum_{i=1}^{n} D_{i},
$$

$\overline{D^{e s t}}$ and $\bar{D}$ represent the average of the estimated and predefined ("true") diffusivities, respectively, in the entire research area.

This correlation coefficient describes the structural similarity between two distribution images (Wang et al. 2004, Eskicioglu and Fisher 1995), and ranges from -1 to 1 . ( $C=1$ if the two images are completely identical, $C=0$ if they are uncorrelated, and $C=-1$ if they are anti-correlated.)

\subsection{Kaczmarz's Method}

Kaczmarz's method is an iterative algorithm for solving linear equation systems. It was first proposed by the Polish mathematician Stefan Kaczmarz (Kaczmarz 1937) and is widely used in computed tomography and signal processing (Lo and Inderwiesen 1994, Elble et al. 2010).

Let $A x=b$ be a linear equation system, where $A$ is an $m \times n$ matrix, $b$ is an $m$ dimensional column vector and $x$ is an $n$-dimensional column vector. Let $x^{0}$ be an arbitrary initial vector. For iteration index $k=0,1, \cdots$, the solution can be approached as 


$$
x^{k+1}=x^{k}+\frac{b_{i}-\left\langle a_{i}, x^{k}\right\rangle}{\left\|a_{i}\right\|^{2}} a_{i}
$$

where $i=k \bmod m, i=1,2, \cdots, m, a_{i}$ is the $i$ th row of matrix $A$, and $b_{i}$ is the $i$ th element of vector $b$.

Let $x^{*}$ be a solution (if one exists), so that the generated sequence $\left\{x^{k}\right\}$ converges to $x^{*}$. Furthermore, if $x^{0}$ is located in the column space of $A^{T}$, then $x^{*}$ is the solution of the minimum 2-norm (Hansan and Saxild-Hansan 2012, Eggermont et al. 1981).

\subsection{The travel times for West-East profile}

Table 7.1. The obtained travel times for West-East profile, [second].

\begin{tabular}{|c|c|}
\hline \multicolumn{2}{|c|}{ Source W14 } \\
\hline Receiver & $\mathrm{t} 100$ \\
\hline E14 & 0.739 \\
\hline E13 & 0.776 \\
\hline E12 & 0.82 \\
\hline E11 & 1.002 \\
\hline E10 & 1.145 \\
\hline E9 & 1.161 \\
\hline E8 & 0.687 \\
\hline E7 & 0.666 \\
\hline E6 & 0.832 \\
\hline E5 & 0.874 \\
\hline E4 & 0.971 \\
\hline E3 & 1.207 \\
\hline E2 & 1.35 \\
\hline E1 & 1.409 \\
\hline Source W10 \\
\hline Receiver & $\mathrm{t} 100$ \\
\hline E14 & 0.91 \\
\hline E13 & 0.932 \\
\hline E12 & 0.941 \\
\hline E11 & 0.86 \\
\hline E10 & 0.668 \\
\hline
\end{tabular}

\begin{tabular}{|c|c|}
\hline \multicolumn{2}{|c|}{ Source W13 } \\
\hline Receiver & $\mathrm{t} 100$ \\
\hline E14 & 0.808 \\
\hline E13 & 0.854 \\
\hline E12 & 0.898 \\
\hline E11 & 1.151 \\
\hline E10 & 1.292 \\
\hline E9 & 1.248 \\
\hline E8 & 0.634 \\
\hline E7 & 0.63 \\
\hline E6 & 0.865 \\
\hline E5 & 0.938 \\
\hline E4 & 1.03 \\
\hline E3 & 1.33 \\
\hline E2 & 1.515 \\
\hline E1 & 1.585 \\
\hline Source W9 \\
\hline Receiver & t100 \\
\hline E14 & 1.088 \\
\hline E13 & 1.085 \\
\hline E12 & 0.972 \\
\hline E11 & 0.693 \\
\hline E10 & 0.349 \\
\hline \multicolumn{2}{|c|}{} \\
\hline \multicolumn{2}{|c|}{}
\end{tabular}

\begin{tabular}{|c|c|}
\hline \multicolumn{2}{|c|}{ Source W12 } \\
\hline Receiver & $\mathrm{t} 100$ \\
\hline E14 & 0.887 \\
\hline E13 & 0.938 \\
\hline E12 & 0.981 \\
\hline E11 & 1.164 \\
\hline E10 & 1.222 \\
\hline E9 & 1.154 \\
\hline E8 & 0.418 \\
\hline E7 & 0.417 \\
\hline E6 & 0.63 \\
\hline E5 & 0.736 \\
\hline E4 & 0.919 \\
\hline E3 & 1.113 \\
\hline E2 & 1.252 \\
\hline E1 & 1.339 \\
\hline Source W8 \\
\hline Receiver & $\mathrm{t} 100$ \\
\hline E14 & 1.123 \\
\hline E13 & 1.121 \\
\hline E12 & 1.1 \\
\hline E11 & 0.7 \\
\hline E10 & 0.349 \\
\hline
\end{tabular}

\begin{tabular}{|c|c|}
\hline \multicolumn{2}{|c|}{ Source W11 } \\
\hline Receiver & $\mathrm{t} 100$ \\
\hline E14 & 0.866 \\
\hline E13 & 0.922 \\
\hline E12 & 0.968 \\
\hline E11 & 1.071 \\
\hline E10 & 0.96 \\
\hline E9 & 0.571 \\
\hline E8 & 0.197 \\
\hline E7 & 0.195 \\
\hline E6 & 0.281 \\
\hline E5 & 0.329 \\
\hline E4 & 0.426 \\
\hline E3 & 0.625 \\
\hline E2 & 0.742 \\
\hline E1 & 0.832 \\
\hline Source W7 \\
\hline Receiver & $\mathrm{t} 100$ \\
\hline E14 & 1.135 \\
\hline E13 & 1.135 \\
\hline E12 & 1.119 \\
\hline E11 & 0.923 \\
\hline E10 & 0.518 \\
\hline
\end{tabular}




\begin{tabular}{|c|c|} 
E9 & 0.292 \\
\hline E8 & 0.091 \\
\hline E7 & 0.09 \\
\hline E6 & 0.148 \\
\hline E5 & 0.198 \\
\hline E4 & 0.256 \\
\hline E3 & 0.364 \\
\hline E2 & 0.628 \\
\hline E1 & 0.703 \\
\hline Source
\end{tabular}

Source W6

\begin{tabular}{|c|c|}
\hline Receiver & $\mathrm{t} 100$ \\
\hline E14 & 1.192 \\
\hline E13 & 1.191 \\
\hline E12 & 1.178 \\
\hline E11 & 1.058 \\
\hline E10 & 0.583 \\
\hline E9 & 0.336 \\
\hline E8 & 0.158 \\
\hline E7 & 0.157 \\
\hline E6 & 0.246 \\
\hline E5 & 0.279 \\
\hline E4 & 0.334 \\
\hline E3 & 0.548 \\
\hline E2 & 0.598 \\
\hline E1 & 0.618 \\
\hline Soure
\end{tabular}

Source W2

\begin{tabular}{|c|c|}
\hline Receiver & $\mathrm{t} 100$ \\
\hline E14 & 2.143 \\
\hline E13 & 2.139 \\
\hline E12 & 2.121 \\
\hline E11 & 1.885 \\
\hline E10 & 1.515 \\
\hline E9 & 1.041 \\
\hline E8 & 0.672 \\
\hline E7 & 0.67 \\
\hline E6 & 0.723 \\
\hline E5 & 0.721 \\
\hline E4 & 0.742 \\
\hline E3 & 0.76 \\
\hline E2 & 0.772 \\
\hline E1 & 0.763 \\
\hline
\end{tabular}

\begin{tabular}{|c|c|}
\hline E9 & 0.208 \\
\hline E8 & 0.006 \\
\hline E7 & 0.006 \\
\hline E6 & 0.09 \\
\hline E5 & 0.117 \\
\hline E4 & 0.154 \\
\hline E3 & 0.296 \\
\hline E2 & 0.398 \\
\hline E1 & 0.46 \\
\hline Source W5 \\
\hline Weceiver & t100 \\
\hline E14 & 1.7 \\
\hline E13 & 1.699 \\
\hline E12 & 1.687 \\
\hline E11 & 1.526 \\
\hline E10 & 0.984 \\
\hline E9 & 0.602 \\
\hline E8 & 0.29 \\
\hline E7 & 0.29 \\
\hline E6 & 0.452 \\
\hline E5 & 0.476 \\
\hline E4 & 0.515 \\
\hline E3 & 0.575 \\
\hline E2 & 0.619 \\
\hline E1 & 0.633 \\
\hline E2 & 0.217 \\
\hline E1 & 0.012 \\
\hline Source W4 & 0.101 \\
\hline Eeceiver & t100 \\
\hline E14 & 1.853 \\
\hline E13 & 1.848 \\
\hline E12 & 1.823 \\
\hline E11 & 1.363 \\
\hline E10 & 1.069 \\
\hline E9 & 0.762 \\
\hline E8 & 0.338 \\
\hline E7 & 0.338 \\
\hline E6 & 0.433 \\
\hline E5 & 0.452 \\
\hline E4 & 0.525 \\
\hline E3 & 0.65 \\
\hline E2 & 0.7 \\
\hline E1 & 0.709 \\
\hline
\end{tabular}

\begin{tabular}{|c|c|}
\hline E9 & 0.277 \\
\hline E8 & 0.101 \\
\hline E7 & 0.099 \\
\hline E6 & 0.168 \\
\hline E5 & 0.212 \\
\hline E4 & 0.262 \\
\hline E3 & 0.493 \\
\hline E2 & 0.583 \\
\hline E1 & 0.617 \\
\hline Source W3 \\
\hline Receiver & t100 \\
\hline E14 & 2.238 \\
\hline E13 & 2.235 \\
\hline E12 & 2.217 \\
\hline E11 & 1.685 \\
\hline E10 & 1.35 \\
\hline E9 & 0.904 \\
\hline E8 & 0.566 \\
\hline E7 & 0.567 \\
\hline E6 & 0.664 \\
\hline E5 & 0.672 \\
\hline E4 & 0.707 \\
\hline E3 & 0.754 \\
\hline E2 & 0.781 \\
\hline E1 & 0.782 \\
\hline
\end{tabular}

\begin{tabular}{|c|c|}
\hline \multicolumn{2}{|c|}{ Source W1 } \\
\hline Receiver & $\mathrm{t} 100$ \\
\hline E14 & 2.302 \\
\hline E13 & 2.297 \\
\hline E12 & 2.275 \\
\hline E11 & 1.831 \\
\hline E10 & 1.459 \\
\hline E9 & 1.117 \\
\hline E8 & 0.68 \\
\hline E7 & 0.678 \\
\hline E6 & 0.715 \\
\hline E5 & 0.71 \\
\hline E4 & 0.726 \\
\hline E3 & 0.733 \\
\hline E2 & 0.74 \\
\hline E1 & 0.727 \\
\hline
\end{tabular}




\subsection{The travel times for North-South profile}

Table 7.2. The obtained travel times for North-South profile, [second].

\begin{tabular}{|c|c|}
\hline \multicolumn{2}{|c|}{ Source N14 } \\
\hline Receiver & $\mathrm{t} 100$ \\
\hline S14 & 0.639 \\
\hline S13 & 0.712 \\
\hline S12 & 0.878 \\
\hline S11 & 0.926 \\
\hline S10 & 0.994 \\
\hline S9 & 0.921 \\
\hline S8 & 0.652 \\
\hline S7 & 0.645 \\
\hline S6 & 0.849 \\
\hline S5 & 1.021 \\
\hline S4 & 1.045 \\
\hline S3 & 1.144 \\
\hline S2 & 1.295 \\
\hline S1 & 1.366 \\
\hline Source N10 \\
\hline Receiver & $\mathrm{t} 100$ \\
\hline S14 & 0.839 \\
\hline S13 & 0.849 \\
\hline S12 & 0.862 \\
\hline S11 & 0.861 \\
\hline S10 & 0.418 \\
\hline S9 & 0.262 \\
\hline S8 & 0.157 \\
\hline S7 & 0.153 \\
\hline S6 & 0.232 \\
\hline S5 & 0.391 \\
\hline S4 & 0.417 \\
\hline S3 & 0.523 \\
\hline S2 & 0.727 \\
\hline S1 & 0.788 \\
\hline Source N6 \\
\hline Receiver & $\mathrm{t} 100$ \\
\hline S14 & 0.907 \\
\hline S13 & 0.875 \\
\hline
\end{tabular}

\begin{tabular}{|c|c|}
\hline \multicolumn{2}{|c|}{ Source N13 } \\
\hline Receiver & $\mathrm{t} 100$ \\
\hline S14 & 0.615 \\
\hline $\mathrm{S} 13$ & 0.651 \\
\hline S12 & 0.726 \\
\hline S11 & 0.776 \\
\hline S10 & 0.883 \\
\hline S9 & 0.742 \\
\hline $\mathrm{S} 8$ & 0.599 \\
\hline S7 & 0.594 \\
\hline S6 & 0.689 \\
\hline S5 & 0.936 \\
\hline S4 & 0.985 \\
\hline S3 & 1.164 \\
\hline S2 & 1.382 \\
\hline S1 & 1.442 \\
\hline \multicolumn{2}{|c|}{ Source N9 } \\
\hline Receiver & $\mathrm{t} 100$ \\
\hline S14 & 0.699 \\
\hline $\mathrm{S} 13$ & 0.675 \\
\hline $\mathrm{S} 12$ & 0.62 \\
\hline S11 & 0.562 \\
\hline S10 & 0.208 \\
\hline S9 & 0.089 \\
\hline $\mathrm{S} 8$ & 0.002 \\
\hline S7 & 0.002 \\
\hline S6 & 0.07 \\
\hline S5 & 0.175 \\
\hline S4 & 0.204 \\
\hline S3 & 0.293 \\
\hline S2 & 0.548 \\
\hline S1 & 0.592 \\
\hline \multicolumn{2}{|c|}{ Source N5 } \\
\hline Receiver & $\mathrm{t} 100$ \\
\hline S14 & 1.108 \\
\hline $\mathrm{S} 13$ & 1.083 \\
\hline $\mathrm{S} 12$ & 1.025 \\
\hline S11 & 0.956 \\
\hline
\end{tabular}

\begin{tabular}{|c|c|}
\hline \multicolumn{2}{|c|}{ Source N12 } \\
\hline Receiver & $\mathrm{t} 100$ \\
\hline S14 & 0.635 \\
\hline $\mathrm{S} 13$ & 0.669 \\
\hline $\mathrm{S} 12$ & 0.748 \\
\hline S11 & 0.804 \\
\hline $\mathrm{S} 10$ & 0.876 \\
\hline S9 & 0.658 \\
\hline S8 & 0.521 \\
\hline S7 & 0.516 \\
\hline S6 & 0.6 \\
\hline S5 & 0.832 \\
\hline S4 & 0.915 \\
\hline S3 & 1.112 \\
\hline $\mathrm{S} 2$ & 1.249 \\
\hline S1 & 1.301 \\
\hline \multicolumn{2}{|c|}{ Source N8 } \\
\hline Receiver & $\mathrm{t} 100$ \\
\hline S14 & 0.82 \\
\hline $\mathrm{S} 13$ & 0.76 \\
\hline $\mathrm{S} 12$ & 0.549 \\
\hline S11 & 0.474 \\
\hline $\mathrm{S} 10$ & 0.162 \\
\hline S9 & 0.088 \\
\hline S8 & 0.009 \\
\hline S7 & 0.002 \\
\hline S6 & 0.074 \\
\hline S5 & 0.135 \\
\hline S4 & 0.156 \\
\hline S3 & 0.373 \\
\hline S2 & 0.471 \\
\hline S1 & 0.514 \\
\hline \multicolumn{2}{|c|}{ Source N4 } \\
\hline Receiver & $\mathrm{t} 100$ \\
\hline S14 & 1.196 \\
\hline $\mathrm{S} 13$ & 1.177 \\
\hline $\mathrm{S} 12$ & 1.13 \\
\hline S11 & 1.077 \\
\hline
\end{tabular}

\begin{tabular}{|c|c|}
\hline \multicolumn{2}{|c|}{ Source N11 } \\
\hline Receiver & $\mathrm{t} 100$ \\
\hline S14 & 0.865 \\
\hline $\mathrm{S} 13$ & 0.9 \\
\hline $\mathrm{S} 12$ & 0.958 \\
\hline $\mathrm{S} 11$ & 0.986 \\
\hline $\mathrm{S} 10$ & 0.935 \\
\hline S9 & 0.459 \\
\hline S8 & 0.322 \\
\hline S7 & 0.318 \\
\hline S6 & 0.407 \\
\hline S5 & 0.683 \\
\hline S4 & 0.766 \\
\hline S3 & 0.974 \\
\hline S2 & 1.123 \\
\hline S1 & 1.169 \\
\hline \multicolumn{2}{|c|}{ Source N7 } \\
\hline Receiver & $\mathrm{t} 100$ \\
\hline S14 & 0.818 \\
\hline $\mathrm{S} 13$ & 0.755 \\
\hline $\mathrm{S} 12$ & 0.567 \\
\hline S11 & 0.491 \\
\hline S10 & 0.168 \\
\hline S9 & 0.091 \\
\hline S8 & 0.009 \\
\hline S7 & 0.002 \\
\hline S6 & 0.077 \\
\hline S5 & 0.142 \\
\hline S4 & 0.164 \\
\hline S3 & 0.371 \\
\hline S2 & 0.488 \\
\hline S1 & 0.531 \\
\hline \multicolumn{2}{|c|}{ Source N3 } \\
\hline Receiver & $\mathrm{t} 100$ \\
\hline S14 & 1.364 \\
\hline $\mathrm{S} 13$ & 1.308 \\
\hline $\mathrm{S} 12$ & 1.213 \\
\hline S11 & 1.145 \\
\hline
\end{tabular}




\begin{tabular}{|c|c|}
\hline S10 & 0.296 \\
\hline S9 & 0.131 \\
\hline S8 & 0.09 \\
\hline S7 & 0.088 \\
\hline S6 & 0.121 \\
\hline S5 & 0.304 \\
\hline S4 & 0.328 \\
\hline S3 & 0.417 \\
\hline S2 & 0.533 \\
\hline S1 & 0.587 \\
\hline Source N2 \\
\hline Receiver & $\mathrm{t} 100$ \\
\hline S14 & 1.583 \\
\hline S13 & 1.517 \\
\hline S12 & 1.396 \\
\hline S11 & 1.323 \\
\hline S10 & 0.787 \\
\hline S9 & 0.637 \\
\hline S8 & 0.551 \\
\hline S7 & 0.546 \\
\hline S6 & 0.601 \\
\hline S5 & 0.648 \\
\hline S4 & 0.661 \\
\hline S3 & 0.706 \\
\hline S2 & 0.722 \\
\hline S1 & 0.72 \\
\hline & \\
\hline
\end{tabular}

\begin{tabular}{|c|c|c|c|}
\hline S10 & 0.502 & $\mathrm{~S} 10$ & 0.587 \\
\hline S9 & 0.265 & S9 & 0.313 \\
\hline S8 & 0.194 & S8 & 0.245 \\
\hline S7 & 0.192 & S7 & 0.242 \\
\hline S6 & 0.258 & S6 & 0.295 \\
\hline S5 & 0.38 & S5 & 0.485 \\
\hline S4 & 0.432 & S4 & 0.521 \\
\hline S3 & 0.539 & S3 & 0.591 \\
\hline S2 & 0.581 & S2 & 0.621 \\
\hline S1 & 0.59 & S1 & 0.628 \\
\hline
\end{tabular}

\begin{tabular}{|c|c|}
\hline $\mathrm{S} 10$ & 0.648 \\
\hline $\mathrm{S} 9$ & 0.503 \\
\hline $\mathrm{S} 8$ & 0.334 \\
\hline $\mathrm{S} 7$ & 0.33 \\
\hline $\mathrm{S} 6$ & 0.454 \\
\hline $\mathrm{S} 5$ & 0.543 \\
\hline $\mathrm{S} 4$ & 0.558 \\
\hline $\mathrm{S} 3$ & 0.609 \\
\hline $\mathrm{S} 2$ & 0.631 \\
\hline $\mathrm{S} 1$ & 0.634 \\
\hline
\end{tabular}

Source N1

\begin{tabular}{|c|c|}
\hline Receiver & $\mathrm{t} 100$ \\
\hline $\mathrm{S} 14$ & 1.661 \\
\hline $\mathrm{S} 13$ & 1.62 \\
\hline $\mathrm{S} 12$ & 1.492 \\
\hline $\mathrm{S} 11$ & 1.367 \\
\hline $\mathrm{S} 10$ & 0.833 \\
\hline $\mathrm{S} 9$ & 0.636 \\
\hline $\mathrm{S} 8$ & 0.554 \\
\hline $\mathrm{S} 7$ & 0.55 \\
\hline $\mathrm{S} 6$ & 0.595 \\
\hline $\mathrm{S} 5$ & 0.635 \\
\hline $\mathrm{S} 4$ & 0.647 \\
\hline $\mathrm{S} 3$ & 0.687 \\
\hline $\mathrm{S} 2$ & 0.696 \\
\hline $\mathrm{S} 1$ & 0.69 \\
\hline
\end{tabular}

